\title{
A New Generation of Chirped Pulse Rotational Spectroscopy with Applications to Structure Determination and Astrochemistry
}

\author{
Justin Lindsay Neill \\ Hillsborough, North Carolina
}

B.S., Davidson College, 2006

\begin{abstract}
A Dissertation presented to the Graduate Faculty
of the University of Virginia in Candidacy for the Degree of

Doctor of Philosophy
\end{abstract}

Department of Chemistry

University of Virginia

December, 2011

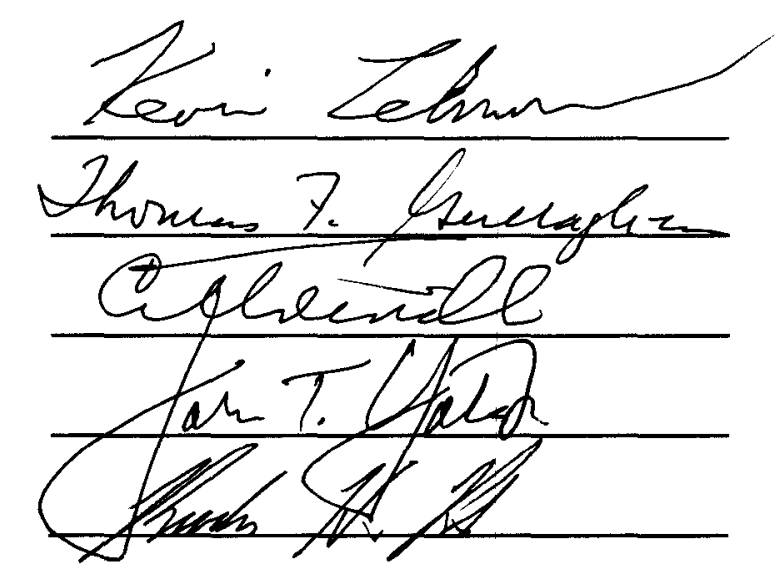




\section{Abstract}

Chirped-pulse rotational spectroscopy is emerging as a powerful technique for molecular detection and characterization. Using this broadband technique, a multicomponent mixture can be quantitatively analyzed quickly, and the unique characteristics of rotational spectroscopy allow for the structural determination of all of the species in the sample. In the past few years, instrumental upgrades have made a qualitatively new class of problems accessible to chirped-pulse rotational spectroscopy. The instrumental improvements made in our laboratory in the past few years, along with techniques to aid in the assignment and characterization of complex spectra, are described.

The new capabilities of this spectrometer are applied to two types of problems. The first is in the direct structure determination of large molecules and molecular clusters through the measurement of isotopically labeled species, particularly in the size regime (>10 non-hydrogen atoms) where weak interactions play a crucial role in overall structure, and where electronic structure theory begins to struggle in accurately capturing the effects of various competing forces. The second application is to astrochemistry, where broadband spectroscopic techniques are needed to partner with broadband radio astronomical facilities in understanding the complex chemistry that occurs in the interstellar medium. This thesis will also describe ways in which the principles of chirped-pulse spectroscopy can be applied in the millimeter and submillimeter regions, which open up a variety of both research and commercial applications. 


\section{Table of Contents}

Chapter 1: Introduction...........................................................1

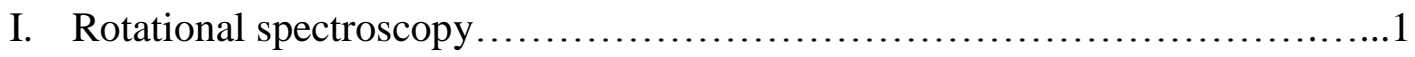

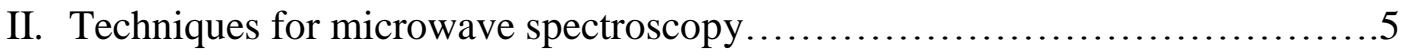

III. Applications of chirped-pulse FTMW spectroscopy...................... 12

Chapter 1 References..............................................16

\section{Chapter 2: Development of a Direct Digital 2-8 GHz Chirped-Pulse Fourier}

Transform Microwave Spectrometer........................................19

I. Introduction..................................................... 19

II. Experimental and Computational Methods..............................23

a) $2-8 \mathrm{GHz}$ CP-FTMW spectrometer.............................23

b) CP-FTMW-MW double resonance spectroscopy.....................26

c) Chemical methods and electronic structure calculations................27

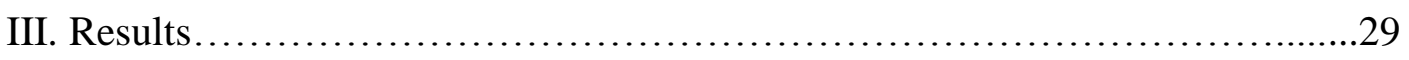

a) CP-FTMW spectrum of iodobenzene and spectrometer performance...........................................29

b) CP-FTMW-MW double resonance spectroscopy....................36

c) Spectrum and structure of the iodobenzene - neon complex.............38

IV. Conclusion......................................................47

Chapter 2 References............................................49 


\section{Chapter 3: Application of High-Sensitivity CP-FTMW Spectroscopy to the}

Structural Determination of Large Molecules and Clusters........................53

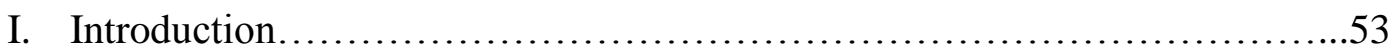

II. Spectrometer Design and Techniques for Spectral Analysis...................55

a) High-sensitivity 7.0-18.5 GHz CP-FTMW spectroscopy...............55

b) Reduced-bandwidth CP-FTMW spectroscopy for deeper averaging.....61

c) CP-FTMW Stark effect measurements..............................64

d) Microwave-microwave double resonance spectroscopy.................66

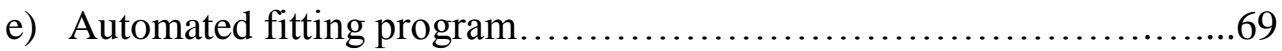

III. Structure Determination of a Large, Floppy Molecule: Dispersive Interactions in

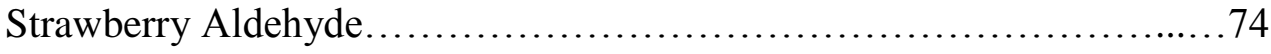

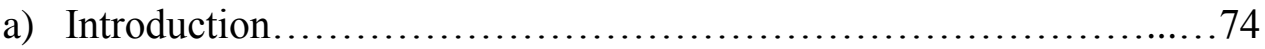

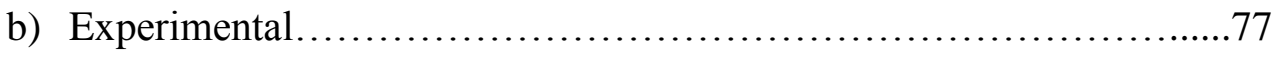

c) Structural characterization of the ${\mathrm{c}-\mathrm{sg}^{-}}^{-}$and $\mathrm{t}$-aa conformers...............78

d) Potential energy surface of strawberry aldehyde........................84

e) Conclusion............................................................

IV. Structure Determination of Clusters of Formic Acid............................91

a) Introduction.................................................... 91

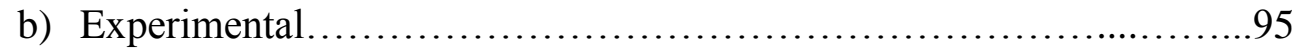

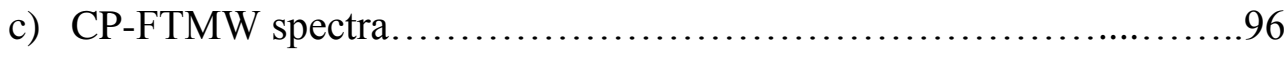

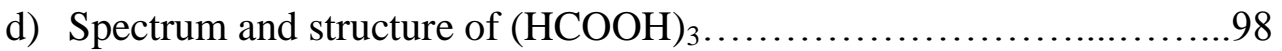

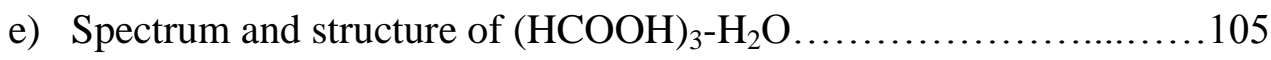




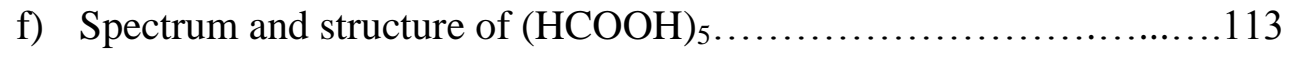

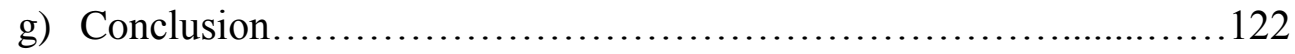

Chapter 3 References............................................ 123

Chapter 4: Spatial Distributions and Interstellar Reaction Processes...............127

I. Introduction...................................................... 127

II. Results......................................................... 132

a) Chemical images from radio telescope arrays......................132

b) Interpretation of chemical images based on chemical reaction

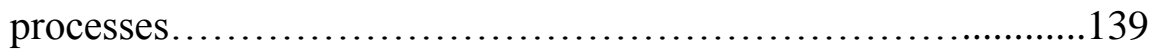

c) Methyl formate production via acid-catalyzed Fischer esterification............................................ 143

d) Methyl formate production via methyl cation transfer.................145

e) Dissociation of protonated methyl formate....................... 148

III. Discussion..................................................... 152

IV. Conclusion ................................................... 155

Chapter 4 References........................................... 157

Chapter 5: Laboratory Detection of trans-Methyl Formate and trans-gauche-Ethyl Formate, and Interstellar Detection of trans-Methyl Formate.................163

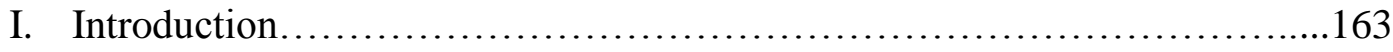

II. Experimental.................................................. 164

III. Laboratory Detection of trans-Methyl Formate......................... 167

IV. Laboratory Detection of trans-gauche-Ethyl Formate..................... 178 
a) Potential energy surface of ethyl formate.........................178

b) Microwave spectrum and Hamiltonian of trans-gauche-ethyl formate.

V. Interstellar Detection of trans-Methyl Formate 195

a) Column density and temperature determination 195

b) Discussion. 204

VI. Conclusion. .208

Chapter 5 References 212

Chapter 6: Techniques for High-Bandwidth ( $\geq 30$ GHz) Chirped-Pulse Millimeter

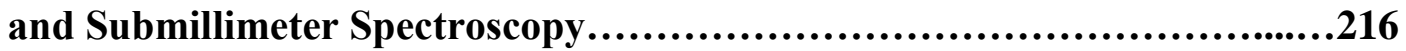

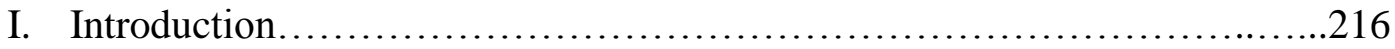

II. Spectrometer Design..............................................223

III. Chirped-Pulse Frequency Combs for Absorption Spectroscopy..............225

IV. Segmented Chirped-Pulse Fourier Transform Emission Spectroscopy........236

V. Conclusions and Future Directions.....................................242

Chapter 6 References..............................................246

Chapter 7: Conclusions.........................................................249

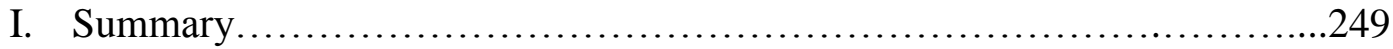

II. Future Developments...........................................250

Chapter 7 References............................................253 


\section{Acknowledgements}

No individual can write a successful Ph.D. thesis without a great deal of help, and I certainly cannot claim to be an exception. I have been fortunate to work alongside many very good scientists in the Pate Lab, each of whom have diverse backgrounds and strengths, and each of whom I am fortunate to call my friend. The research presented here that I consider to be exciting and worthwhile would not be possible without the contributions of the other Pate Lab members I have worked with, both former (Gordon Brown, Kevin Douglass, Steve Shipman, Leonardo Alvarez-Valtierra) and current (Matt Muckle, Danny Zaleski, Amanda Steber, Brent Harris, Nathan Seifert), along with a number of undergraduate students. Much of my thesis is written with an eye towards even better developments to come in the next few years, which I know the current and future members of the lab will implement successfully in ways that cannot even be foreseen now. I also do not have room to acknowledge here by name each of the collaborators from other groups at UVa and other institutions that I have worked with here, but their contributions also have been invaluable.

Of course the strong science presented here would also not be possible without the leadership and guidance of my advisor, Brooks Pate. I have been fortunate to be able to (attempt to) model my thought process after a scientist who thinks deeply about each aspect of each experiment and system that the group studies, and who refuses to settle for the accepted norms of the field, but instead seeks new avenues of research that have yet to be considered. He sets the bar very high for any research scientist. 
My time at the University has also been made richer by my experience as a Jefferson Fellow. The financial support of my graduate study, as well as personal support from Doug Trout and the staff at the Jefferson Scholars Foundation, and the experience of interacting with and discussing research with other Fellows and other people associated with the Foundation, has been extremely valuable. The support of the staff in the Chemistry Department has also made all aspects of research and life as a graduate student easier.

Finally, the support of my family has been irreplaceable. My wife, Claire, has provided nothing but support, whether research was going well or not, whether I was home or not. My parents, Anne and Lindsay, as well as my wonderful in-laws, Ruth and Jim, have all teamed together to make a full-time support staff without which I don't know how I could have made it through this process. This thesis is what it is only because of the support and love of all of you. 


\section{Chapter 1}

\section{Introduction}

\section{Rotational spectroscopy}

Molecular rotational spectroscopy has a long history as a powerful technique for

general chemical analysis and characterization. ${ }^{1-3}$ Any gas-phase molecule with a permanent dipole moment has a characteristic fingerprint pure rotational spectrum in the microwave and millimeter-wave frequency region, arising from the quantization of angular momentum, and, therefore, the rotational energy that a quantum mechanical system can possess. The spectrum is obtained through the interaction of a molecular sample with a field of electromagnetic radiation, which can cause the molecule's rotational energy to change when its frequency corresponds to the energy of a quantummechanically allowed transition between rotational states, related by the Bohr condition ( $\Delta E=h v$, where $h$ is Planck's constant).

The frequencies of the transitions in this spectrum, as well as their relative intensities, are related to calculable structural and electronic parameters of the molecule. Analysis of a spectrum is performed by fitting a set of observed transition frequencies to those predicted by a model Hamiltonian. The primary values determined in an analysis of the spectrum are the rotational constants, which are inversely related to the molecule's moments of inertia:

$$
A=\frac{h}{8 \pi^{2} I_{a}}, \quad B=\frac{h}{8 \pi^{2} I_{b}}, \quad C=\frac{h}{8 \pi^{2} I_{c}}
$$


where $a, b$, and $c$ are the principal axes of the molecule. The principal axes are defined so that the off-diagonal terms in the inertial tensor, the products of inertia, are zero. The axes, by convention, are labeled so that $A \geq B \geq C$.

In addition to the rotational constants, a number of other properties of a molecule can be derived from an analysis of its rotational spectrum. In a perfectly rigid rotor, the frequencies of the rotational spectrum would be described completely by the rotational constants, but a real molecule is distortable, and therefore most rotational spectral analyses must include centrifugal distortion corrections. Other parameters can be included in the Hamiltonian, depending on the properties of the molecule under study. Nuclear and electronic spins can couple with the rotational angular momentum of the molecule and induce splitting in rotational transitions, an analysis of which can yield information about the electronic properties of the system. If the molecule can undergo internal motion, such as with the internal rotation of a methyl rotor, this internal angular momentum couples to the overall rotational angular momentum and introduces additional terms to the rotational Hamiltonian; a variety of approaches have been developed for characterizing these types of systems. ${ }^{4}$ A number of freely available programs have been developed to fit complex rotational spectra. ${ }^{5-7}$

There is typically a large degree of redundancy in rotational spectra; that is, the ratio of observed transitions to fit parameters in a spectral analysis is typically 10 or greater. Because of this, and because of the high precision to which rotational transition frequencies can be measured by modern spectroscopic techniques (typically $\Delta v / v \leq 10^{-6}$ ), molecular parameters can be determined with extremely high precision. This is 
important for one of the most powerful applications of pure rotational spectroscopy, that of structural determination. This is accomplished through the assignment of isotopically substituted species of a molecule. Through the application of Kraitchman's equations, ${ }^{8}$ from the changes to the rotational constants induced by the isotopic substitution at a single atom, the position of that atom in the molecule's principal axis system can be derived. By assigning the spectra of isotopically substituted species at each position in a molecule, the structure of the molecule can be built up atom by atom, deriving the socalled $r_{\mathrm{s}}$ structure. The common organic heavy (i.e., non-hydrogen) atoms $(\mathrm{C}, \mathrm{N}, \mathrm{O})$ each have minor isotopes at $0.2-1 \%$ of the abundance of the primary isotope, meaning that this kind of analysis can often be performed without resorting to isotopically enriched samples, which for larger molecules are typically not available, and can be synthetically challenging. In the Kraitchman structure analysis, it is assumed that the vibrationally averaged structure of each isotopologue is the same; however, the change in mass induced by isotopic substitution changes the vibrational frequencies, and therefore the zero-point vibrational wavefunctions of each isotopologue are different. This is particularly important to keep in mind for larger molecules with low-frequency vibrational modes, which have larger zero-point amplitudes. Limitations of the Kraitchman analysis have been thoroughly discussed elsewhere. ${ }^{9}$

Alternatively, the structure of a molecule can be derived using rotational constants by fitting the moments of inertia of multiple isotopes of a molecule to internal parameters (bond lengths, angles, and dihedral angles); this is used to derive the $r_{0}$ structure of the molecule (the ground state vibrationally averaged structure). ${ }^{10-12}$ Vibrational corrections, 
which are often obtained through high-level $a b$ initio calculations, can be included to derive the $r_{\mathrm{e}}$ structure (i.e. the true minimum-energy structure with no vibrational motion), and a number of analyses of this sort have been performed. ${ }^{13-15}$ The derivation of an $r_{\mathrm{e}}$ structure, however, requires expensive electronic structure calculations, as well as a great deal of reliance on the accuracy of these calculations, and therefore has so far been performed only for relatively small molecules (15 atoms or less).

The intensities of the observed transitions depend on the magnitude and orientation of the permanent dipole moment in the principal axis system. Transitions of an asymmetric top (i.e., a molecule where $A \neq B \neq C$ ) are divided into $a$-type, $b$-type, and $c$-type transitions, which are so named because their observation requires that $\mu_{\mathrm{a}} \neq 0$, $\mu_{\mathrm{b}} \neq 0$, and $\mu_{\mathrm{c}} \neq 0$, respectively, where $\mu_{\mathrm{a}}, \mu_{\mathrm{b}}$, and $\mu_{\mathrm{c}}$ are the projections of the molecule's permanent dipole moment on the principal axes. The relative intensities of the three types of transitions are proportional to the squares of the dipole moment components, and so a rough estimate of the relative ratios of $\mu_{\mathrm{a}}, \mu_{\mathrm{b}}$, and $\mu_{\mathrm{c}}$ can be obtained from the relative intensities of transitions in the spectrum. The magnitude of the dipole moment can be determined through the Stark effect, the shifts of rotational energy levels under the presence of an applied electric field.

The favorable characteristics of rotational spectroscopy described above make it a highly useful technique for unambiguous molecular detection, as well as a tool for investigating molecular structural properties. The intensities and linewidths of the transitions in a molecule's rotational spectrum also depend on the conditions of the molecule. The transition intensities also depend on the populations of the various energy 
states of the molecule, from which the molecule's rotational temperature can be determined. The linewidths can be determined by a number of broadening effects, the most common of which are Doppler (velocity), collisional, or lifetime broadening. These aspects of rotational spectroscopy make it the most powerful method for observing molecules in interstellar space. ${ }^{16}$ In addition to the unambiguity of a molecular detection made by rotational spectroscopy, due to the large number of transitions that can be observed, and the unique nature of the rotational spectrum, observations of interstellar molecules, made by radio astronomical telescopes, yield information about both the physical conditions, such as the temperature and kinematics, of astronomical regions where chemistry is occurring.

\section{Techniques for microwave spectroscopy}

The development of microwave radar technology during World War II led to the first measurements of molecular rotational spectra. Early spectrometers in the microwave region were based on measuring transitions through their absorption of radio frequency radiation. The Stark-effect microwave spectrometer developed in the late $1940 \mathrm{~s}^{17,18}$ used a modulated DC electric field to vary the absorption of the gas sample, introducing a frequency component to the transmitted microwave power that could be amplified to generate a molecular signal. This spectrometer design was used with great success to study a wide variety of molecular systems, including systems with multiple conformational isomers,${ }^{19}$ nuclear quadrupole coupling, ${ }^{20}$ and internal rotation. ${ }^{21}$ 
Room-temperature microwave spectroscopy is challenging due to the fact for most molecules with sufficient vapor pressure to be studied in a static gas cell, the frequency at which the intensity of the rotational spectrum peaks is typically in the millimeter/submillimeter region, rather than in the microwave. The development of jetcooled molecular beams for high-resolution spectroscopy, as first presented by Smalley, Wharton, and Levy, ${ }^{22}$ enabled the preparation of rotationally and vibrationally cold molecular samples. As the rotational partition function scales with $T^{1.5}$ for nonlinear molecules ( $T$ for linear molecules), the benefit from cooling samples to a few degrees Kelvin is considerable. Additionally, this cooling has the benefit of moving the Boltzmann intensity peak into the microwave for most molecules, greatly enhancing the sensitivity of spectroscopic techniques in this region. Pulsed-jet microwave spectroscopy was developed in the late 1970s by the Flygare group, ${ }^{23-27}$ using Fourier transform microwave (FTMW) techniques which, rather than measuring the molecular absorption of radiation, instead used short coherent excitation pulses to create macroscopic polarizations in a gas sample, followed by the background-free, time-domain measurement of the free induction decay (FID) signal that results as these polarizations decay. The observed FID signal is then Fourier transformed to yield the frequency spectrum of the sample. These techniques are largely rooted in analogous techniques for nuclear magnetic resonance (FT-NMR). ${ }^{28}$ Because the molecular emission signals are detected against zero background, rather than as small changes against a strong electric field, emission spectroscopy has generally been demonstrated to be more sensitive, and yield higher frequency resolution, than absorption techniques. 
The Balle-Flygare spectrometer design ${ }^{27}$ further enhanced the sensitivity of this technique by introducing a Fabry-Perot resonator to amplify the pulse used for sample polarization and the molecular signals that result. This basic design, with a variety of improvements made over the years, ${ }^{29,30}$ is still used in many laboratories for highsensitivity, high-resolution spectroscopy today. This spectrometer has been particularly useful in the spectroscopic characterization of weakly bound molecular complexes, which are stabilized in pulsed supersonic expansion sources; the structure and dynamics of a wide variety of molecular complexes have been characterized using pulsed-jet FTMW spectroscopy, ${ }^{31,32}$ which has provided a vast amount of experimental information to the understanding of weak intermolecular forces. This spectrometer has been coupled to a variety of molecular sources, including laser ablation sources to study metal-containing compounds $^{33,34}$ and biologically important molecules, ${ }^{35,36}$ and discharge sources ${ }^{37,38}$ to synthesize molecules of atmospheric ${ }^{39}$ and interstellar interest. ${ }^{40,41}$

The primary limitation of the Balle-Flygare FTMW spectrometer design is its small frequency bandwidth on a single measurement. As discussed above, a typical rotational spectrum contains a large number of transitions, and determination of structural parameters to high precision requires the measurement of many transitions, which is experimentally time-consuming in a scanning spectrometer. Additionally, in many microwave spectroscopic studies, the rotational constants, and therefore the frequencies of transitions in the rotational spectrum, are not well known before experimental measurement, so large bandwidth scans are needed. Without broadband spectral information, other interesting species such as clusters or weak conformational isomers 
that were not initially anticipated in the sample can be missed. Even with automated scanning techniques that have been implemented into these spectrometers, ${ }^{29}$ even relatively low-sensitivity survey scans are time consuming: at a typical cavity mode width of $\sim 500 \mathrm{kHz}$, with 20 averages per frequency step and a $10 \mathrm{~Hz}$ pulsed nozzle repetition rate, the spectrometer can only scan at a rate of $900 \mathrm{MHz} / \mathrm{hr}$ (not including the time required to move the cavity mirrors and change the microwave components for each step). This is particularly problematic when using samples that are expensive, such as biologically important molecules, or with molecular generation sources that can change with time.

To overcome this limitation, and to open up new applications for molecular rotational spectroscopy, chirped-pulse Fourier transform microwave (CP-FTMW) spectroscopy ${ }^{42,43}$ has been developed in recent years as a powerful method for obtaining broadband molecular spectra. The foundation for CP-FTMW spectroscopy was laid in the $1970 \mathrm{~s}$, when fast passage excitation was shown by McGurk et al. ${ }^{44}$ and Wolf $\mathrm{W}^{45}$ to be an efficient way to excite a molecular sample. The implementation of time-domain, broad-bandwidth chirped-pulse microwave spectroscopy, however, required the development of high-bandwidth sources for the generation of chirped pulses and digitizers that could detect large spectral bandwidths. Both of these have become available in recent years with technological advances spurred by the telecommunications industry.

Chirped pulses provide efficient excitation of a molecular sample because they offer independent control of the duration and bandwidth of the pulse. A transform- 
limited pulse can achieve increased bandwidth only by shortening its time duration, which reduces the overall energy delivered to the sample. A chirped pulse, having the general form

$$
E(t)=E_{0} \cos \left(\omega_{0} t+\frac{1}{2} \alpha t^{2}\right)
$$

executes a linear frequency sweep beginning at frequency $\omega_{0}$ and proceeding with sweep rate $\alpha$; that is, the instantaneous frequency of the pulse at any time is

$$
\omega(t)=\omega_{0}+\alpha t
$$

The efficiency of chirped-pulse excitation for large-bandwidth microwave spectroscopy as compared to transform-limited pulses has been demonstrated. ${ }^{43}$ The specific designs and applications of CP-FTMW spectrometers operating in frequency ranges from $2 \mathrm{GHz}$ into the millimeter/submillimeter will be described in this thesis.

For the vast majority of systems, the measurement time and sample consumption required to acquire a broadband spectrum is greatly reduced in CP-FTMW spectroscopy as compared to a Balle-Flygare cavity spectrometer. Because of the large bandwidth excited by each pulse, the CP-FTMW spectrometer uses standard gain horn antennas (cavity quality factor $Q=1$ ) instead of a Fabry-Perot resonator $\left(Q \sim 10^{4}\right.$ or more). Because of this, the single-shot signal to noise of the CP-FTMW spectrometer with a single pulsed nozzle is lower than that of the Balle-Flygare design by approximately a factor of 50. The exact factor depends on the dipole moment of the molecule under study, because the Balle-Flygare spectrometer typically achieves optimal " $\pi / 2$ " excitation, while the CP-FTMW spectrometer does not due to the large bandwidths addressed by the excitation pulses; therefore, the signal levels of the two spectrometers 
scale differently with the dipole moment of the molecule. Since the signal-to-noise ratio using either spectrometer increases with the square root of the number of signal averages performed in an experiment, this means that approximately 2,500 CP-FTMW signal averages would be required to achieve the sensitivity achieved in a single pulse with the Balle-Flygare design.

However, this is compensated for by the fact that the cavity must make many frequency steps to achieve the same bandwidth as a CP-FTMW spectrometer addresses on each measurement cycle; for a $10 \mathrm{GHz}$ spectrum, for example, with a $500 \mathrm{kHz}$ frequency step, the cavity must perform 20,000 measurements to acquire the same bandwidth. Therefore, with a single pulsed nozzle, the CP-FTMW spectrometer requires nearly an order of magnitude less time to acquire a broadband spectrum, and consumes an order of magnitude less sample. Additionally, multiple pulsed nozzle sources can be implemented in the CP-FTMW spectrometer to increase sensitivity. For the 7.0-18.5 GHz spectrometer, currently three pulsed nozzles, which must be separated by $20 \mathrm{~cm}$ to avoid interference between the expansions, are simultaneously pulsed on each valve cycle. This increases the observed molecular signals by a factor of three, due to the coherent addition of the signal of each molecule due to the $N$-emitter effect. ${ }^{46}$ This is more efficient for both time and sample consumption than pulsing a single nozzle, which would require nine times as many pulses to achieve the same sensitivity as the threenozzle configuration.

Additionally, the relative intensity accuracy in CP-FTMW spectroscopy is very good (within 20-25\%), as has been demonstrated previously ${ }^{43}$ and will be demonstrated 
as well in this thesis, across several orders of magnitude of transition intensity. This is crucial because the relative intensities between transitions are fixed by the relative dipole moment components of the molecule and the transition dipole matrix elements and, therefore, are of great utility in making spectral assignments when they are reliable. In the assignment of isotopically substituted species for structural determination, the relative intensities are highly useful in screening for potential spectral assignments in dense spectra. Finally, for the quantitative characterization of samples where multiple species are present, whether they are conformational isomers of a large molecule, the products of a discharge, or a gas mixture, accurate relative transition intensities are crucial in determining the relative abundances of different species. The relative intensity accuracy of cavity FTMW spectrometers is typically unreliable, because the performance of the resonator can vary widely as a function of frequency. Also, depending on where each molecular transition falls within the cavity mode, its intensity can vary widely.

In the past few years, we have developed high-sensitivity techniques for CPFTMW spectroscopy to improve its ability to measure weak transitions. These are focused on the implementation of multiple nozzles as discussed above, and the application of multiple microwave pulses, and the detection of multiple FIDs, on each valve injection cycle. These enhancements have enabled the routine detection of ${ }^{13} \mathrm{C}$ and ${ }^{18} \mathrm{O}$ isotopologues in natural abundance, even for molecules and clusters with 10 or more heavy atoms. To accompany this improvement in sensitivity, we have worked to develop techniques for the fitting of the dense spectra that the CP-FTMW spectrometer is capable 
of obtaining. These techniques, and demonstrations of their utility for using CP-FTMW spectroscopy to understand molecular structure, will be described in this thesis.

\section{Applications of chirped-pulse FTMW spectroscopy}

This thesis presents the application of high-sensitivity CP-FTMW spectroscopy to two basic areas of research in chemistry. The first is in the application of microwave spectroscopy to the structural determination of large molecules and molecular clusters. As one seeks to study the spectra of larger molecules, it is desirable to measure spectra at lower frequency because the Boltzmann intensity peak moves to lower frequency with increasing size. In Chapter 2, a direct digital, 2-8 GHz CP-FTMW spectrometer is presented, along with its application to the spectrum of iodobenzene. The microwave spectra of the ${ }^{13} \mathrm{C}$ isotopologues of iodobenzene were identified in natural abundance for the first time in this study, along with the spectrum of the complex of iodobenzene with a neon atom; using isotopic substitution as described above, structural information is derived for both iodobenzene and the iodobenzene-neon complex.

In Chapter 3, the application of CP-FTMW spectroscopy to the structure determination of large molecules and weakly bound complexes will be explored. The challenges associated with these studies, the techniques that have been developed in recent years, and the structural information that can be determined are discussed. These tools are applied to the conformational energy surface of strawberry aldehyde. This molecule, which contains 15 heavy atoms, has a large number of possible conformational isomers, of which 5 were identified in the supersonic expansion. For two of these 
conformers, each ${ }^{13} \mathrm{C}$ isotopologue was assigned in natural abundance, from which an experimental structures was derived. Certain conformers of this molecule also exhibit a long-range dispersion interaction between ends of the molecule, which poses a challenge for electronic structure theory to describe accurately. This project shows that microwave spectroscopy can provide an important role in testing structures of large molecules predicted by theoretical calculations through the direct structural information that can be obtained.

The application of high-sensitivity CP-FTMW spectroscopy to structural studies of molecular complexes will then be described, using complexes of formic acid. These complexes are of great interest to the chemical physics community due to the role of formic acid dimer as a benchmark system for proton tunneling dynamics. ${ }^{47}$ Using CPFTMW spectroscopy, the structures of three new complexes involving formic acid have been experimentally determined: formic acid trimer, the complex of three formic acid molecules with a single water molecule, and formic acid pentamer. Techniques for the assignment of spectra, particularly weak isotopologues, and for structural derivation from the observed data will be described.

The second focus of this thesis will be in the application of CP-FTMW spectroscopy to interstellar chemistry. Chapters 4 and 5 will discuss how multispecies molecular spatial maps, obtained using interferometric observatories, can suggest possible reactions to form complex molecules. Formation processes of methyl formate, a highly abundant interstellar molecule for which these pathways are not well understood, are explored by comparing its spatial distribution in the Orion molecular cloud to that of 
three related molecules: methanol, formic acid, and dimethyl ether. Based on these spatial distributions, two new gas-phase ion-molecule reactions between methanol and formic acid, where one of the reactants is protonated, are proposed that could lead to interstellar methyl formate and explain the observed relative spatial distributions. A first test of these reaction proposals is performed using CP-FTMW spectroscopy. The proposed gas-phase reactions, and particularly the most favorable one that can occur without a barrier, suggest that the less stable conformational isomer (trans) of methyl formate could be formed at a detectable abundance by this chemistry. Based on the potential energy surface of neutral methyl formate, it is likely that any trans-methyl formate formed as a result of a kinetically favorable process would not be converted to the more stable cis form rapidly, due to the presence of a large barrier to their interconversion.

Using CP-FTMW spectroscopy in conjunction with a high-sensitivity, highresolution Balle-Flygare-type spectrometer and a microwave-microwave double resonance spectrometer, the first assignment of the rotational spectra of both trans-methyl formate and trans-gauche-ethyl formate, which could form through an analogous process, are presented. Both species are complicated by internal motion effects. Using the rotational line frequencies obtained by CP-FTMW spectroscopy and a publicly available survey of a different chemically rich interstellar source, the Sagittarius B2(N) molecular cloud, the first interstellar identification of trans-methyl formate is presented. This isomer is seen at a far higher abundance than would be expected under local thermodynamic equilibrium. Interestingly, it is also seen that trans-methyl formate has a 
dramatically lower rotational temperature than the more stable, more abundant cis isomer. Possible interpretations of the interstellar observation of trans-methyl formate, as well as the physical conditions of the two isomers in this region, will be discussed.

Chapter 6 will describe recent work towards the application of chirped pulse spectroscopy to the large-bandwidth (30 GHz or greater) acquisition of molecular spectra in the millimeter and submillimeter wavelength regions $(\sim 40 \mathrm{GHz}-3 \mathrm{THz})$. Several millimeter wave telescope facilities have come online in the last few years or are nearing completion which will provide unprecedented sensitivity, frequency and spatial resolution, and bandwidth to investigate the chemistry of the Universe, and laboratory techniques will be needed that can match the capabilities of these facilities. Techniques utilizing chirped pulse radiation fields, generated by an AWG as for CP-FTMW spectroscopy and multiplied into the millimeter/submillimeter by high-power active multiplier chains, will be described that have the potential to offer orders-of-magnitude enhancement of the speed with which these spectra can be obtained. These techniques offer great promise for the future of laboratory rotational spectroscopy for the measurement of molecules of interstellar interest, as well as for general chemical analysis. 


\section{Chapter 1 References}

(1) Townes, C. H., Schawlow, A.L. Microwave Spectroscopy; Dover: New York, 1975.

(2) Woolrab, J. E. Rotational Spectra and Molecular Structure; Academic: New York, 1967.

(3) Gordy, W., Cook, R.L. Microwave Molecular Spectra; John Wiley and Sons: New York, 1984.

(4) Kleiner, I. J. Mol. Spectrosc. 2010, 260, 1-18.

(5) Kisiel, Z. PROSPE, Programs for ROtational SPEctroscopy, http://info.ifpan.edu.pl/ kisiel/prospe.htm.

(6) Pickett, H. M. J. Mol. Spectrosc. 1991, 148, 371-377.

(7) Plusquellic, D. F.; Suenram, R. D.; Mate, B.; Jensen, J. O.; Samuels, A. C. J. Chem. Phys. 2001, 115, 3057-3067.

(8) Kraitchman, J. Am. J. Phys. 1953, 21, 17-24.

(9) Demaison, J.; Rudolph, H. D. J. Mol. Spectrosc. 2002, 215, 78-84.

(10) Nosberger, P.; Bauder, A.; Günthard, H. H. Chem. Phys. 1973, 1, 418-425.

(11) Watson, J. K. G.; Roytburg, A.; Ulrich, W. J. Mol. Spectrosc. 1999, 196, 102-119.

(12) Rudolph, H. D. Struct. Chem. 1991, 2, 581-588.

(13) Groner, P.; Warren, R. D. J. Mol. Struct. 2001, 599, 323-335.

(14) Kisiel, Z. J. Mol. Spectrosc. 2003, 218, 58-67.

(15) Craig, N. C.; Groner, P.; McKean, D. C. J. Phys. Chem. A 2006, 110, 7461-7469.

(16) Herbst, E.; van Dishoeck, E. F. Annu. Rev. Astron. Astrophys. 2009, 47, 427-480. 
(17) Hughes, R. H.; Wilson, E. B. Phys. Rev. 1947, 71, 562.

(18) McAfee, J. K. B.; Hughes, R. H.; Wilson, J. E. B. Rev. Sci. Instrum. 1949, 20, 821-826.

(19) Scroggin, D. G.; Riveros, J. M.; Wilson, E. B. J. Chem. Phys. 1974, 60, 13761385.

(20) Azrak, R. G.; Wilson, E. B. J. Chem. Phys. 1970, 52, 5299-5316.

(21) Ford, R. G.; Beaudet, R. A. J. Chem. Phys. 1971, 55, 3110-3113.

(22) Smalley, R. E.; Wharton, L.; Levy, D. H. J. Chem. Phys. 1975, 63, 4977-4989.

(23) Ekkers, J.; Flygare, W. H. Rev. Sci. Instrum. 1976, 47, 448-454.

(24) Balle, T. J.; Campbell, E. J.; Keenan, M. R.; Flygare, W. H. J. Chem. Phys. 1980, $72,922-932$.

(25) Campbell, E. J.; Buxton, L. W.; Balle, T. J.; Flygare, W. H. J. Chem. Phys. 1981, $74,813-828$.

(26) Campbell, E. J.; Buxton, L. W.; Balle, T. J.; Keenan, M. R.; Flygare, W. H. J. Chem. Phys. 1981, 74, 829-840.

(27) Balle, T. J.; Flygare, W. H. Rev. Sci. Instrum. 1981, 52, 33-45.

(28) Gunther, H. NMR Spectroscopy, 2nd ed.; John Wiley \& Sons: New York, 1995.

(29) Grabow, J.-U.; Stahl, W.; Dreizler, H. Rev. Sci. Instrum. 1996, 67, 4072-4084.

(30) Arunan, E.; Dev, S.; Mandal, P. K. Appl. Spectrosc. Rev. 2004, 39, 131 - 181.

(31) Legon, A. C. Annu. Rev. Phys. Chem. 1983, 34, 275-300.

(32) Novick, S. Bibliography of Rotational Spectra of Weakly Bound Complexes, http://www.wesleyan.edu/chem/faculty/novick/vdw.html. 
(33) Cooke, S. A.; Gerry, M. C. L. J. Mol. Spectrosc. 2005, 234, 195-203.

(34) Walker, K. A.; Gerry, M. C. L. J. Mol. Spectrosc. 1997, 182, 178-183.

(35) Lesarri, A.; Mata, S.; Lopez, J. C.; Alonso, J. L. Rev. Sci. Instrum. 2003, 74, 4799-4804.

(36) Sanz, M. E.; Lesarri, A.; Pena, M. I.; Vaquero, V.; Cortijo, V.; Lopez, J. C.; Alonso, J. L. J. Am. Chem. Soc. 2006, 128, 3812-3817.

(37) McCarthy, M. C.; Chen, W.; Travers, M. J.; Thaddeus, P. Astrophys. J. Suppl. Ser. 2000, 129, 611-623.

(38) Ohshima, Y.; Endo, Y. Chem. Phys. Lett. 1996, 256, 635-640.

(39) Suma, K.; Sumiyoshi, Y.; Endo, Y. Science 2005, 308, 1885-1886.

(40) M. Iida, Y. O., Y. Endo. Astrophys. J. 1991, 371, L45-L46.

(41) McCarthy, M. C.; et al. Astrophys. J. Lett. 2006, 652, L141.

(42) Brown, G. G.; Dian, B. C.; Douglass, K. O.; Geyer, S. M.; Pate, B. H. J. Mol. Spectrosc. 2006, 238, 200-212.

(43) Brown, G. G.; Dian, B. C.; Douglass, K. O.; Geyer, S. M.; Shipman, S. T.; Pate, B. H. Rev. Sci. Instrum. 2008, 79, 053103-053113.

(44) McGurk, J. C.; Mader, H.; Hofmann, R. T.; Schmalz, T. G.; Flygare, W. H. J. Chem. Phys. 1974, 61, 3759-3767.

(45) Wolf, F. J. Phys. D: Appl. Phys. 1994, 27, 1774.

(46) Allen, L., Eberly, J.H. Optical Resonance and Two-Level Atoms; Dover: New York, 1987.

(47) Birer, O.; Havenith, M. Annu. Rev. Phys. Chem. 2009, 60, 263-275. 


\section{Chapter 2}

\section{Development of a Direct Digital 2-8 GHz Chirped-Pulse Fourier Transform}

\section{Microwave Spectrometer ${ }^{1}$}

\section{Introduction}

Gas-phase molecular spectroscopy techniques have been increasingly applied to structural studies of large molecules (defined here as having 10 or more non-hydrogen atoms), with a focus on molecules of biological interest. ${ }^{2-6}$ Techniques that are sensitive to molecular shape are desirable for studying large molecules, which often have complex conformational landscapes. As discussed in the Introduction, molecular rotational spectroscopy in the microwave frequency region allows for precise determination of molecular moments of inertia and can be applied to any gas-phase molecule with a permanent dipole moment, and molecular structures can be determined by analyzing the rotational spectra of isotopically substituted species. Comparisons of experimentally and computationally derived molecular structures provide fundamental tests of the quality of various ab initio methods. Another advantage of high-resolution molecular microwave spectroscopy in pulsed jets is the ability to analyze samples that contain a rich mixture of molecular species, including conformers. As a result, rotational spectroscopy is uniquely suited to precision studies of molecular conformational structure where explicit atomic positions are determined.

To study larger systems by rotational spectroscopy, such as molecules of biological interest or containing heavier atoms (such as metals or large halides), it is often desirable to obtain spectra at lower frequencies. The original CP-FTMW design operates 
in the 7.0-18.5 GHz frequency range. However, as a molecule's size increases, its Boltzmann intensity peak at molecular jet temperatures falls into the $2-8 \mathrm{GHz}$ spectral region. This is illustrated in Figure 2.1, where simulated rotational spectra of molecules of increasing size, at a typical pulsed-jet rotational temperature of $1 \mathrm{~K}$, are compared. The rotational spectrum of the lowest-energy conformer (trans) of ethyl formate (panel A, 5 heavy atoms, mass $74.08 \mathrm{u})^{7,8}$ has very little transition intensity in this region. For a larger molecule such as tryptophol (panel B, 12 heavy atoms, $161.20 \mathrm{u}$ ), ${ }^{9}$ there is a great deal more intensity from $2-8 \mathrm{GHz}$, but a large number of strong transitions remain above $8 \mathrm{GHz}$ as well. As molecular size increases further, however, spectrometers below 10 $\mathrm{GHz}$ become essential for pulsed-jet rotational spectroscopy. For example, the calculated spectrum of the tripeptide antioxide glutathione (panel C, 20 heavy atoms, mass $307.32 \mathrm{u})$ has very little transition intensity above $10 \mathrm{GHz}$, and so a high-sensitivity rotational spectroscopy technique for the $2-8 \mathrm{GHz}$ region is needed for pulsed-jet microwave studies of systems of this size.

In a cavity FTMW spectrometer, sensitive operation below $\sim 4 \mathrm{GHz}$ is difficult, as diffraction sets the low-frequency limit of the spectrometer. The original cavity-FTMW spectrometer design of Balle and Flygare contained mirrors with a diameter of $36 \mathrm{~cm}$ and achieved a low-frequency limit of $4.5 \mathrm{GHz}$. One way to improve the low-frequency performance of a cavity FTMW spectrometer is to increase the size of the mirrors; one such spectrometer with mirrors $122 \mathrm{~cm}$ in diameter, operating down to $1 \mathrm{GHz}$, has been recently described. ${ }^{10}$ Specially designed smaller spectrometers have also been able to 


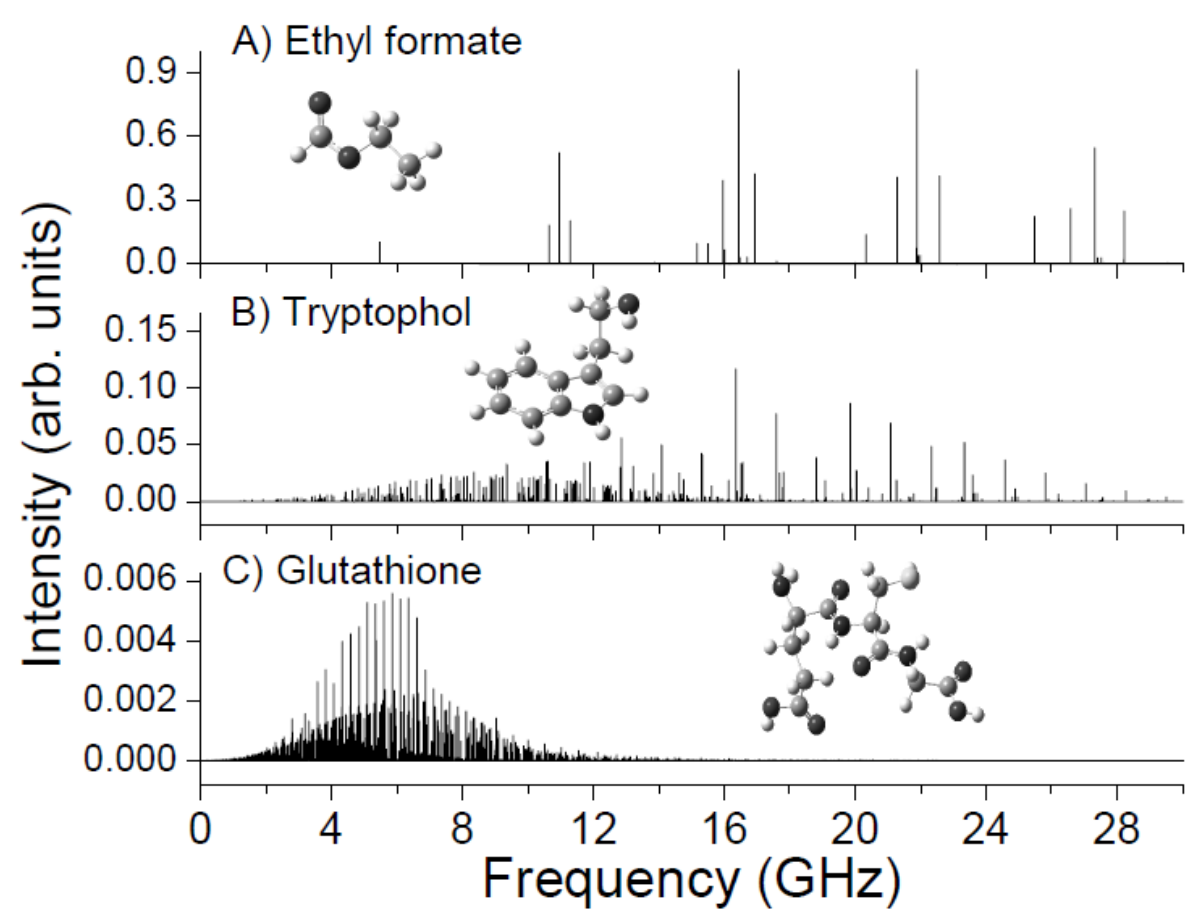

Figure 2.1. Simulated rotational spectra (at a temperature of $1 \mathrm{~K}$ ) of several molecules as a function of size. (A) Most stable conformer (trans) of ethyl formate, using experimental rotational constants $(A=17746.7 \mathrm{MHz}, B=2904.7 \mathrm{MHz}, C=2579.1 \mathrm{MHz})$ and dipole moments $\left.\left(\mu_{a}=1.85 \mathrm{D}, \mu_{b}=0.69 \mathrm{D}\right)\right)^{7,8}$ (B) Observed conformer of tryptophol, using experimental rotational constants $(A=1748.2 \mathrm{MHz}, B=678.0 \mathrm{MHz}, C$ $=549.6 \mathrm{MHz})$ and calculated dipole moments $\left(\mu_{a}=1.6 \mathrm{D}, \mu_{b}=1.9 \mathrm{D}, \mu_{c}=0.5 \mathrm{D}\right){ }^{9}$ (C) One conformer of glutathione, using calculated rotational constants $(A=381.9 \mathrm{MHz}, B=$ 140.6 MHz, $C=111.7 \mathrm{MHz})$ and dipole moments $\left(\mu_{a}=1.3 \mathrm{D}, \mu_{b}=0.6 \mathrm{D}, \mu_{c}=1.9 \mathrm{D}\right)$. 
achieve lower operating frequencies. Storm et al. ${ }^{11}$ designed a cylindrical resonator that operated on the $\mathrm{TE}_{01 q}$ mode, and could operate in the 1-4 $\mathrm{GHz}$ frequency region. Alternatively, Etchison et al. ${ }^{12}$ developed a FTMW cavity spectrometer that could achieve operation in the same frequency range using hemispherical mirrors of radius 40 $\mathrm{cm}$, observing molecular transitions below $1 \mathrm{GHz}$.

In this chapter, the design of a CP-FTMW spectrometer operating in the $2-8 \mathrm{GHz}$ is reported, ideal for larger molecules. Because the technology exists to both directly generate and detect broadband microwave pulses in this frequency region, this spectrometer contains no frequency mixing or multiplication stages. In addition, the spectrometer uses small horn antennas and so there is no need for a large vacuum chamber or pumping system. The utility of this spectrometer has been demonstrated in a recent study of the rotational spectrum of bromoform. ${ }^{13}$ Here the performance of this spectrometer is demonstrated by measuring the spectrum of iodobenzene. In addition to a remeasurement of its rotational spectrum, its four distinct singly substituted ${ }^{13} \mathrm{C}$ isotopologues have been detected, along with the van der Waals cluster of iodobenzene with a neon atom. In addition, the implementation of broadband microwave-microwave double resonance spectroscopy to facilitate spectral assignments is described. 


\title{
II. Experimental and Computational Methods
}

\author{
a) 2-8 GHz CP-FTMW spectrometer
}

A schematic diagram of the 2-8 GHz CP-FTMW spectrometer is shown in Figure 2.2. A linear frequency sweep, typically with a duration of $4 \mu \mathrm{s}$, is created by an arbitrary waveform generator (AWG, Tektronix 7102B with Option 06) with sampling rate $20 \mathrm{GS} / \mathrm{s}$. An $8.5 \mathrm{GHz}, 8$-pole low-pass filter (K\&L Microwave) is placed on the output of the AWG to improve pulse purity. The pulse is then input into a $200 \mathrm{~W}$ pulsed traveling wave tube amplifier (Amplifier Research, 200T2G8A). Figure 2.3 shows a spectrogram of the excitation pulse, both before and after the TWTA. Before TWTA amplification, the only detectable frequency component of the pulse other than the desired frequency output $v(\mathrm{t})$ is due to mixing of the pulse with the $10 \mathrm{GHz}$ clock inside the AWG. The amplifier introduces a few other spurious components, particularly $2 v(t)$ and $3 v(t)$. However, these spurious sweeps are low in intensity $(-10-15 \mathrm{~dB})$ relative to the primary sweep, and have not been observed to affect the relative intensity accuracy of molecular signals observed with this spectrometer, as will be demonstrated below.

The amplified chirped pulse is broadcast into a molecular beam vacuum chamber by a WRD-250 (2.5-7.5 GHz) horn antenna (Advanced Technical Materials, part no. 250441-C3) with 7-13 dBi gain (low to high frequency) and dimensions (face height $\times$ face width $\times$ length) of $13 \times 10 \times 17 \mathrm{~cm}$. The horns were separated by $50 \mathrm{~cm}$ so that two pulsed nozzles, spaced by $20 \mathrm{~cm}$ and oriented perpendicular to the axis of microwave propagation, could be operated simultaneously; this leads to a factor of 4 saved in time 


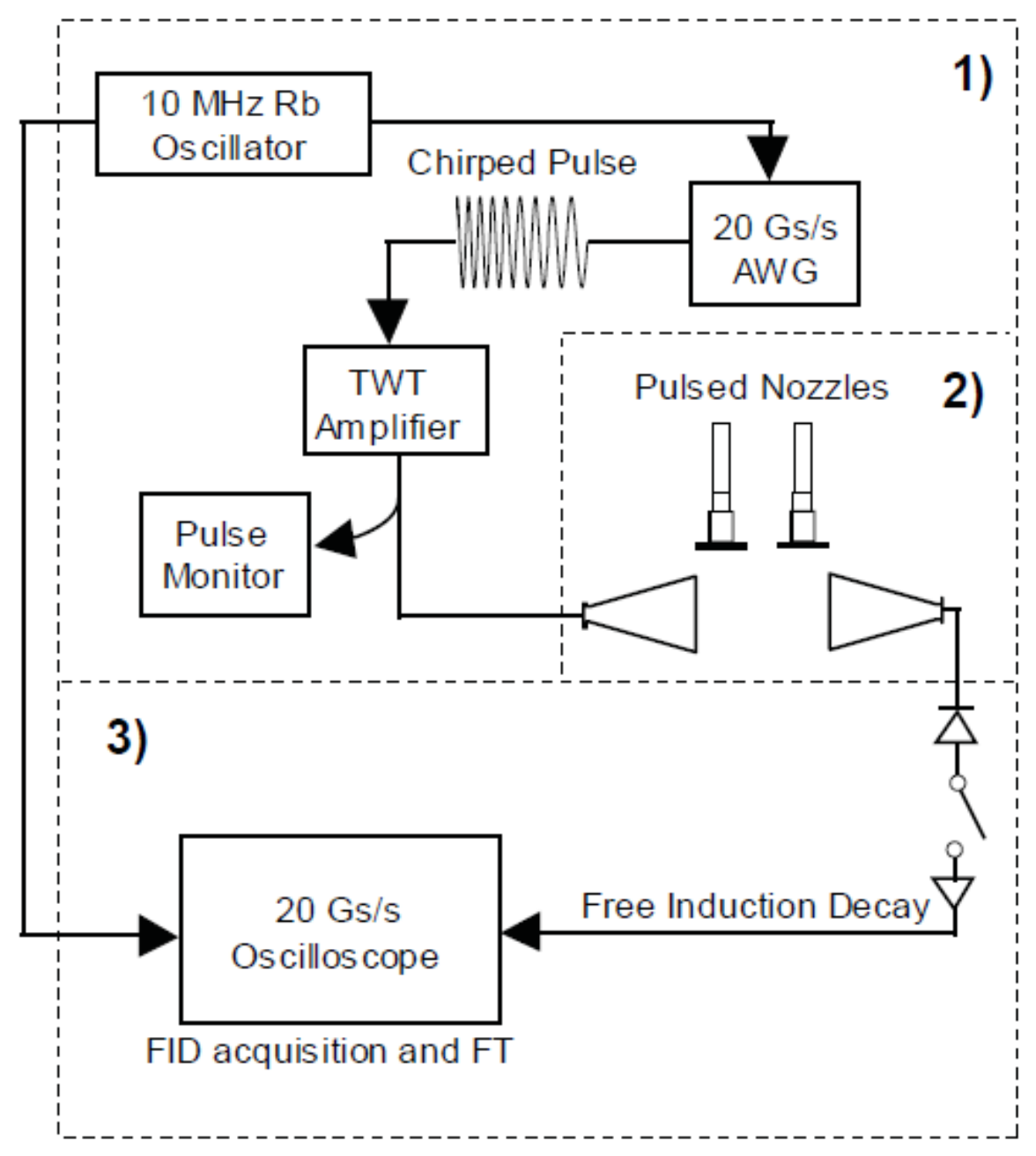

Figure 2.2. Schematic of the $2-8 \mathrm{GHz}$ CP-FTMW spectrometer. The schematic is separated into three sections: (1) pulse generation and amplification; (2) molecular beam chamber; (3) FID amplification and detection. See the text for further description. 

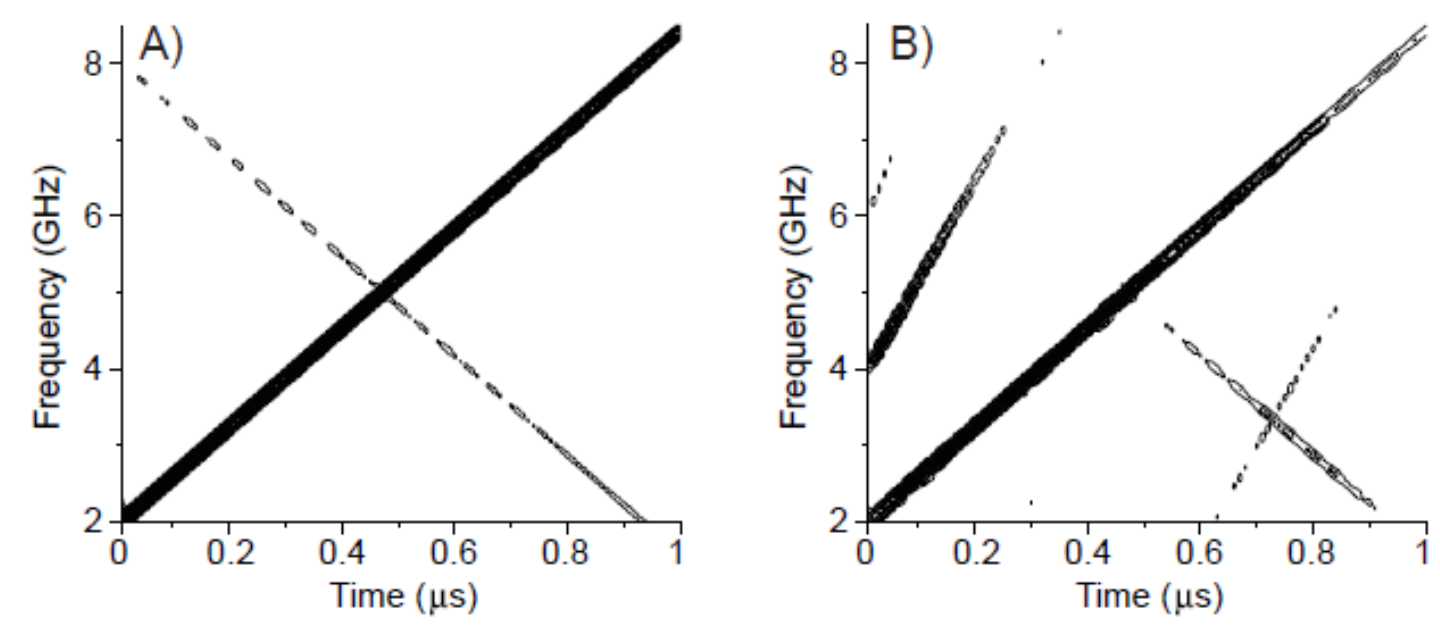

Figure 2.3. Time-frequency analysis of the chirped pulse output of the AWG (panel A) and of the pulse after TWT amplification (panel B). 
and a factor of 2 lower sample consumption. The horn separation distance is set by the directionality of the broadcast pulse, which is lower than that achieved in the higher frequency spectrometer (7.0-18.5 GHz).

To protect the detection electronics from the high-power TWTA pulse, a passive solid state PIN diode limiter (Advanced Control Components, ACLM-4601-1K, 1 kW peak power handling) and a solid state single-pole single-throw (SPST) switch (Advanced Technical Materials, S1517D) are used in front of a high-gain, low-noise five-stage broadband amplifier (Miteq AFS5-00100800-14-10P-5, $40 \mathrm{~dB}$ gain, $1.4 \mathrm{~dB}$ noise figure). The molecular FID signal is directly digitized on a fast oscilloscope (Tektronix TDS6124C, $12 \mathrm{GHz}$ hardware bandwidth) operating at a $20 \mathrm{GS} / \mathrm{s}$ sampling rate. The FID was acquired for $20 \mu$ s to match the typical $T_{2}$ dephasing times of molecular transitions in this frequency range $(\sim 20-25 \mu$ s at the low-frequency end, $\sim 10$ $12 \mu$ s at the high-frequency end). All frequency and trigger sources in the experiment, as well as the digital oscilloscope, are phase-locked to a $10 \mathrm{MHz}$ rubidium-disciplined crystal oscillator to provide phase stability and frequency accuracy.

b) CP-FTMW-MW double resonance spectroscopy

In order to perform CP-FTMW-single frequency $\mathrm{MW}$ double resonance experiments, a single-frequency MW pulse must be generated that is spectrally pure so that "false positives" will not be observed. Because of its spurious frequency outputs and high noise power, a TWTA cannot be used to amplify the single-frequency pulse. Instead, for this pulse we use a $4 \mathrm{~W}, 2-8 \mathrm{GHz}$ solid-state amplifier (SSA, Microwave 
Power Inc., L0208-36-T421) with a TTL driver internally installed. A schematic of the spectrometer in this configuration is shown in Figure 2.4. The AWG creates the pulse sequence, in which the chirped pulse is immediately followed by the single-frequency MW pulse with a sinc intensity profile to provide a highly frequency-selective pulse. The single-frequency MW pulse typically has $4 \mu$ s duration. Using a single-pole doublethrow (SPDT) switch, the chirped pulse is directed to the TWTA as in the normal CPFTMW experiment, but the single-frequency pulse is directed to the SSA. The outputs of the two amplifiers are then combined using a Wilkinson power combiner. To prevent damage to the SSA from TWTA power returned from the power divider, two 2-8 GHz isolators (DiTom Microwave, $10 \mathrm{~dB}$ isolation each) are placed on the output of the SSA. The combined pulse sequence is then broadcast into the vacuum chamber. The SPST detection switch is timed to protect the low-noise amplifier during both microwave pulses.

c) Chemical methods and electronic structure calculations

Iodobenzene (98\% purity) was purchased from Aldrich and used without further purification. The sample was placed in a stainless steel reservoir at room temperature and a backing gas consisting of approximately $70 \%$ neon and $30 \%$ helium ("first run neon") was flowed over it at a backing pressure of $0.5 \mathrm{~atm}$. The mixture was then expanded into the chamber through the pulsed nozzles (General Valve Series 9, $0.9 \mathrm{~mm}$ diameter) with a pulse duration of $500 \mu \mathrm{s}$. A total of 210,000 FIDs were averaged 


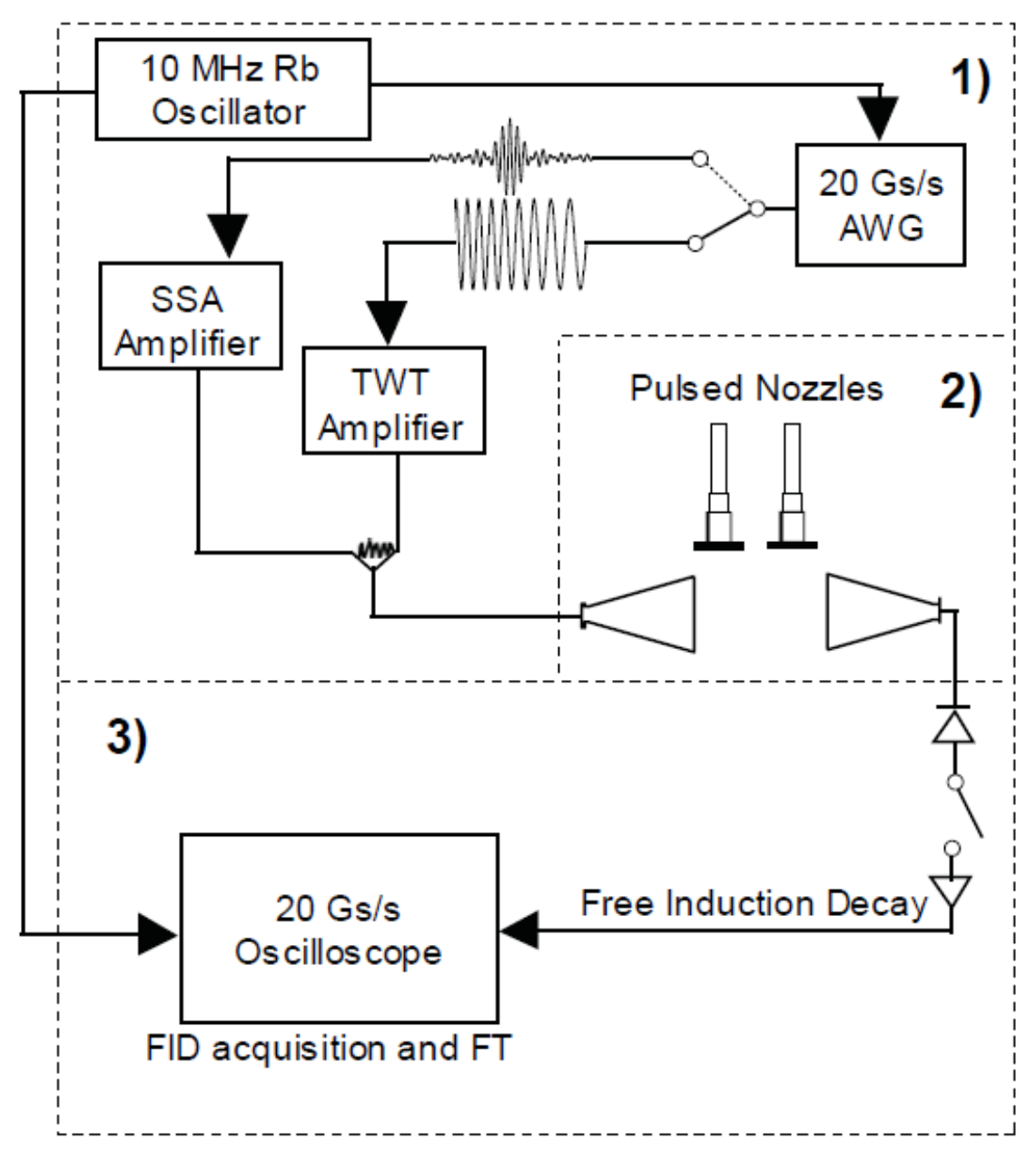

Figure 2.4. The 2-8 GHz CP-FTMW spectrometer as designed to perform microwavemicrowave double resonance experiments. 
together for the final spectrum. A Kaiser-Bessel digital frequency filter ${ }^{14}$ was applied to the FID before Fourier transformation.

$A b$ initio electronic structure calculations of iodobenzene and its neon complex were performed using the Gaussian software package, ${ }^{15}$ at the MP2/6-311G(d,p) level of theory. This basis set for iodine was obtained from the EMSL Basis Set Exchange. ${ }^{16-18}$

\section{Results}

a) CP-FTMW spectrum of iodobenzene and spectrometer performance

The rotational spectrum of iodobenzene has been measured and assigned previously, first in a room-temperature waveguide cell, ${ }^{19}$ then later in a low-frequency FTMW cavity, ${ }^{12}$ followed by further FTMW cavity and millimeter-wave measurements. ${ }^{20}$ In addition, Stark effect measurements in a FTMW cavity were performed to determine its dipole moment, which is oriented along the $a$ principal axis $\left(\mu_{a}=1.6250(20) \mathrm{D}\right) .{ }^{20}$ Its dense rotational spectrum, owing to the large nuclear quadrupole coupling constants of the iodine nucleus, makes it a good benchmark molecule to demonstrate the sensitivity and relative intensity accuracy of the $2-8 \mathrm{GHz}$ CP-FTMW spectrometer.

In the panel (A) of Figure 2.5, the 2-8 GHz CP-FTMW spectrum of iodobenzene is compared to a simulation using the fit Hamiltonian parameters,${ }^{20}$ at a rotational temperature of $1 \mathrm{~K}$. All spectral fitting and simulation in this report was performed using 

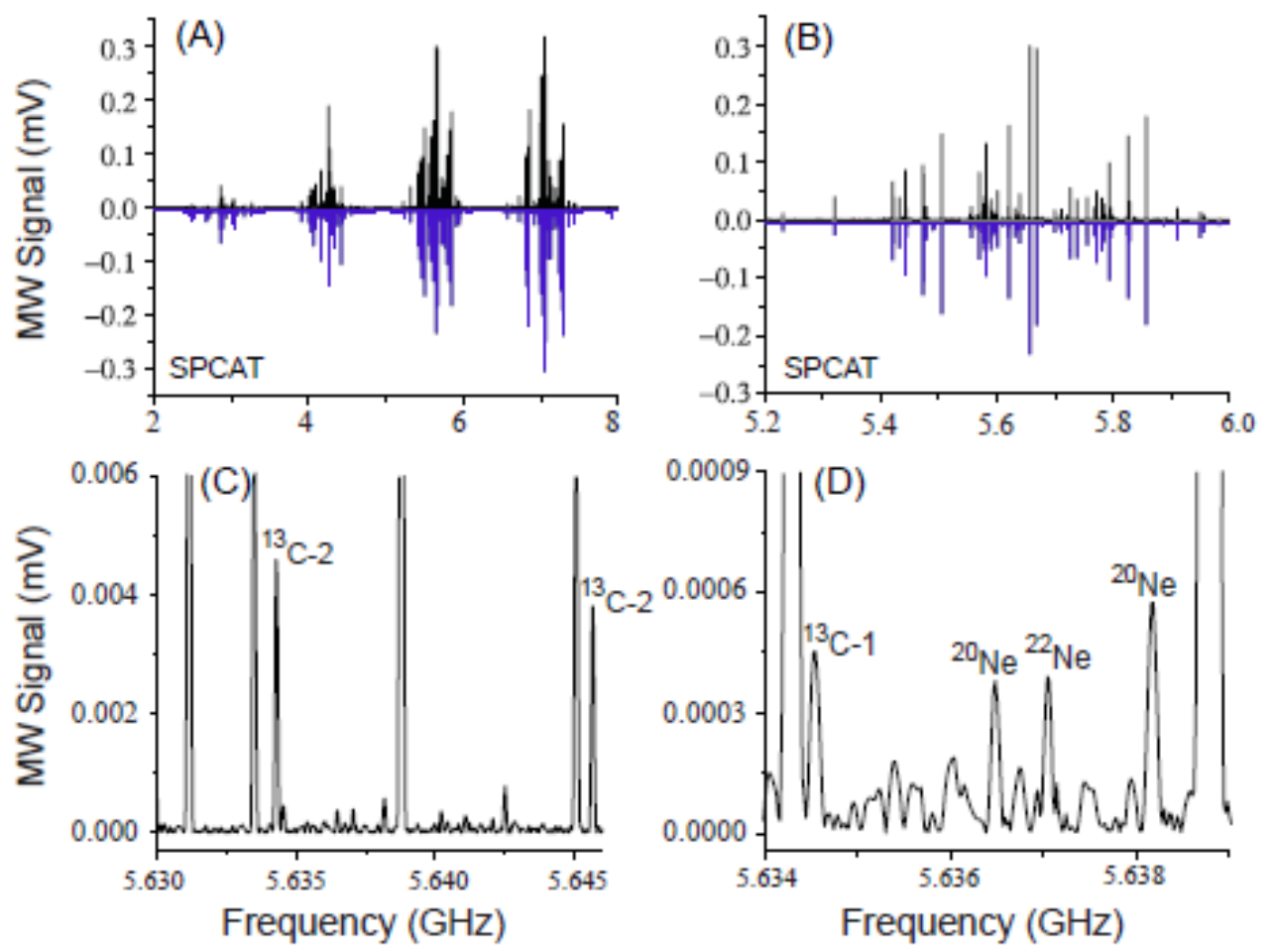

Figure 2.5. The 2-8 GHz CP-FTMW spectrum of iodobenzene after 210,000 FID averages. (A) Comparison of the experimental spectrum (positive-going) to its simulated spectrum at a rotational temperature of $1 \mathrm{~K}$ (negative-going). (B) An expanded view of the $J=4-3$ region is shown to demonstrate the relative intensity accuracy of the spectrometer. (C) An expanded view to show the dynamic range and resolution at baseline of the instrument. The labeled peaks are from the ${ }^{13} \mathrm{C}-2$ isotopomer, and the offscale peaks are normal species transitions. (D) A further expanded view of the spectrum, showing the noise level of the spectrum. Labeled peaks are from the ${ }^{13} \mathrm{C}-1$ isotopomer and the ${ }^{20} \mathrm{Ne}$ and ${ }^{22} \mathrm{Ne}$ clusters. The off-scale peak near $5.634 \mathrm{GHz}$ is from the ${ }^{13} \mathrm{C}-2$ isotopomer, and the off-scale peak near $5.639 \mathrm{GHz}$ is from the normal species of iodobenzene. 
SPFIT/SPCAT, ${ }^{21}$ and PIFORM ${ }^{22}$ was used to reformat the results and determine standard errors for parameters. In panel (B), an expanded view shows the $J=4-3$ region of the spectrum. The results of Watson Hamiltonian fits of the spectrum of iodobenzene are presented in Table 2.1, showing the fit of the CP-FTMW data on its own as well as in conjunction with all previously measured transitions.

In addition, due to the sensitivity of this spectrum, the four distinct carbon-13 isotopomers of iodobenzene are observed in natural abundance. Panels (C) and (D) of Figure 2.5 show expanded views of the CP-FTMW spectrum, with transitions due to these carbon-13 isotopologues indicated. Isolated spectra of these isotopologues are shown in Figure 2.6, compared to simulated spectra. The intensities of these simulations have been scaled from those of the normal species in Figure 2.5 by the known relative abundances of the two carbon isotopes, with those of carbons 2 and 3 doubled due to symmetry. This demonstrates the accuracy of the relative transition intensities of this spectrometer across a large dynamic range. The fit Hamiltonian parameters of these species are found in Table 2.2. It should be noted that although the linewidths obtained in this spectrometer $(\sim 120 \mathrm{kHz}$ FWHM $)$ are larger than those obtained on a BalleFlygare-type spectrometer with a pulsed nozzle parallel to the axis of microwave propagation, the frequency accuracy of the CP-FTMW spectrometer is better than $4 \mathrm{kHz}$ for lines with high signal to noise, and $5-7 \mathrm{kHz}$ for weaker transitions.

From the moments of inertia of the ${ }^{13} \mathrm{C}$-monosubstituted isotopologues, a semiexperimental structure of iodobenzene can be derived. In Table 2.3, the Kraitchman 
Table 2.1. A-reduction Hamiltonian parameters of iodobenzene.

\begin{tabular}{lccc}
\hline & Previous Fit $^{20}$ & Global Fit $^{\mathrm{a}}$ & CP-FTMW Fit \\
\hline$A(\mathrm{MHz})$ & $5669.126(15)$ & $5669.131(14)$ & $5669.252(78)$ \\
$B(\mathrm{MHz})$ & $750.414323(52)$ & $750.414293(43)$ & $750.41443(10)$ \\
$C(\mathrm{MHz})$ & $662.636162(44)$ & $662.636146(38)$ & $662.63646(10)$ \\
& & & \\
$\Delta_{J}(\mathrm{kHz})$ & $0.0195479(14)$ & $0.0195470(14)$ & $0.02794(97)$ \\
$\Delta_{J K}(\mathrm{kHz})$ & $0.164648(67)$ & $0.164636(67)$ & $0.126(12)$ \\
$\Delta_{K}(\mathrm{kHz})$ & $0.891(52)$ & $0.908(50)$ & $0.908^{\mathrm{b}}$ \\
$\delta_{J}(\mathrm{kHz})$ & $0.00253098(65)$ & $0.00253065(63)$ & $0.00253065^{\mathrm{b}}$ \\
$\delta_{K}(\mathrm{kHz})$ & $0.15554(32)$ & $0.15551(32)$ & $0.15551^{\mathrm{b}}$ \\
$\Phi_{J K}(\mathrm{~Hz})$ & $0.0000609(13)$ & $0.0000607(13)$ & \\
$\Phi_{K J}(\mathrm{~Hz})$ & $-0.000337(16)$ & $-0.000378(16)$ & \\
$\chi_{a a}(\mathrm{MHz})$ & $-1892.0398(46)$ & $-1892.0395(22)$ & $-1892.0395(28)$ \\
$\chi_{b b}-\chi_{c c}(\mathrm{MHz})$ & $65.593(12)$ & $65.5872(76)$ & $65.5856(68)$ \\
$M_{a a}(\mathrm{kHz})$ & $-1.67(86)$ & $-1.42(36)$ & \\
$M_{b b}=M_{\mathrm{cc}}(\mathrm{kHz})$ & $-2.60(13)$ & $-2.499(52)$ & $-2.338(70)$ \\
No of lines & 296 & 606 & 310 \\
$\sigma_{\mathrm{w}}$ & 0.793 & 0.799 & 3.7 \\
rms error $(\mathrm{kHz})$ & & &
\end{tabular}

${ }^{a}$ Combined fit of the transitions measured previously and in this study.

${ }^{\mathrm{b}}$ Assumed value. 

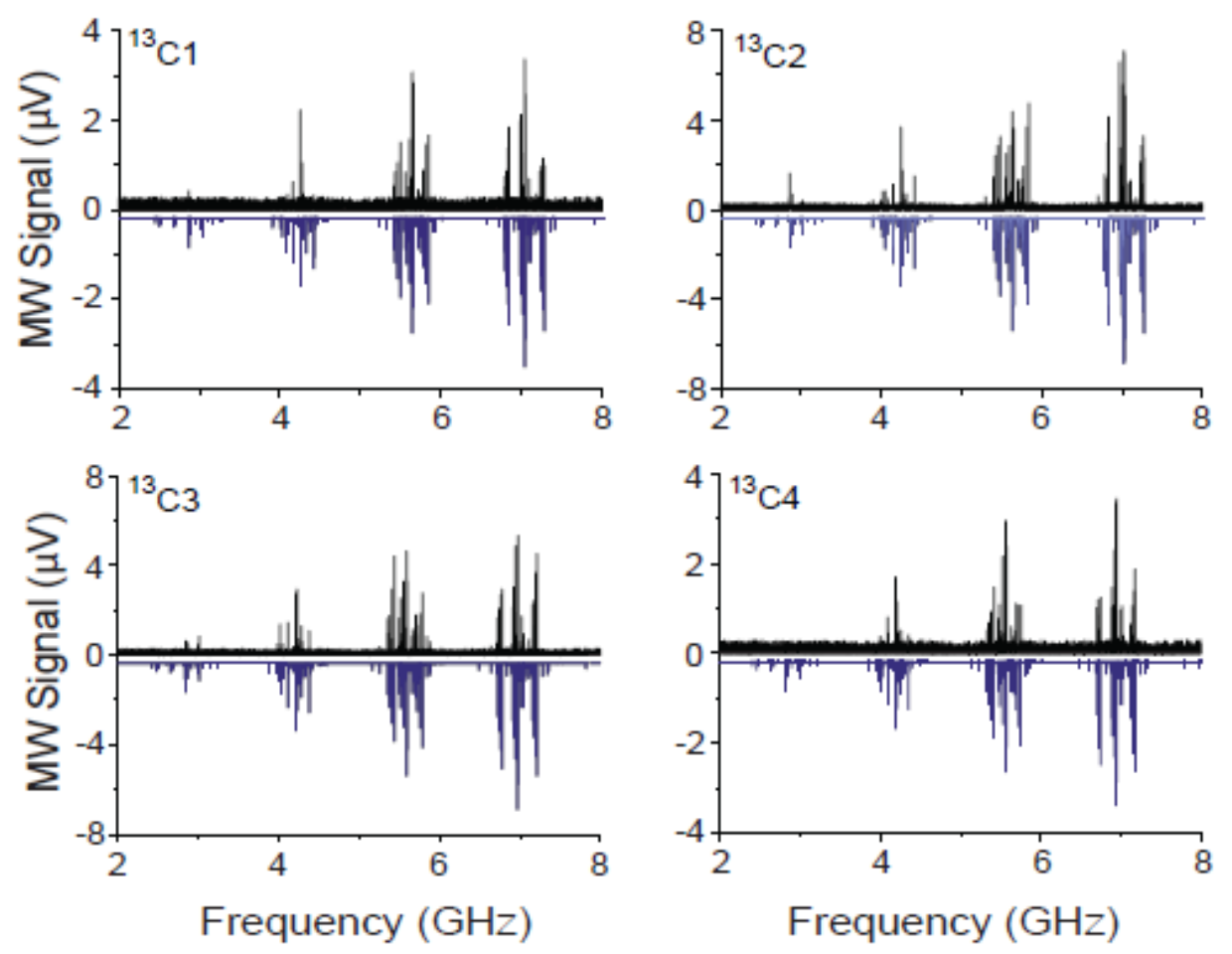

Figure 2.6. Isolated rotational spectra of the four distinct singly substituted ${ }^{13} \mathrm{C}$ isotopologues of iodobenzene, compared to their simulated spectra. These isolated spectra were obtained by removing all transitions not associated with the species of interest from the spectrum. The simulated spectra are scaled from that in Figure 2.5 by the isotopic abundance of ${ }^{13} \mathrm{C}$, with those of $\mathrm{C}_{2}$ and $\mathrm{C}_{3}$ doubled due to symmetry. 
Table 2.2. Fit Hamiltonian parameters of singly-substituted carbon isotopologues of iodobenzene.

\begin{tabular}{lccc}
\hline & Normal Species & ${ }^{13} \mathrm{C}-1^{\mathrm{a}}$ & ${ }^{13} \mathrm{C}-2^{\mathrm{a}}$ \\
\hline$A(\mathrm{MHz})$ & $5669.131(14)$ & $5668.48(46)$ & $5577.33(25)$ \\
$B(\mathrm{MHz})$ & $750.414293(43)$ & $749.80640(31)$ & $748.08728(25)$ \\
$C(\mathrm{MHz})$ & $662.636146(38)$ & $662.16646(28)$ & $659.55540(21)$ \\
$\chi_{\alpha \alpha}(\mathrm{MHz})$ & $-1892.0395(22)$ & $-1892.306(38)$ & $-1891.955(26)$ \\
$\chi_{b b}-\chi_{c c}(\mathrm{MHz})$ & $65.5872(76)$ & $65.54(14)$ & $65.596(52)$ \\
No of lines & 606 & 64 & 76 \\
rms error $(\mathrm{kHz})$ & & 6.6 & 5.6 \\
\hline \multicolumn{3}{c}{${ }^{13} \mathrm{C}-3^{\mathrm{a}}$} & \multicolumn{2}{c}{$\mathrm{C}^{\mathrm{a}}$} \\
\hline$A(\mathrm{MHz})$ & $5579.13(43)$ & $5668.54(34)$ \\
$B(\mathrm{MHz})$ & $741.48220(45)$ & $736.66714(27)$ \\
$C(\mathrm{MHz})$ & $654.44323(39)$ & $651.89770(23)$ \\
$\chi_{\alpha \alpha}(\mathrm{MHz})$ & $-1891.927(43)$ & $-1892.008(35)$ \\
$\chi_{b b}-\chi_{c c}(\mathrm{MHz})$ & $65.68(16)$ & $67.50(14)$ \\
No of lines & 76 & 59 \\
rms error $(\mathrm{kHz})$ & 6.1 & 5.5 \\
\hline
\end{tabular}

${ }^{a}$ All parameters not listed here were fixed to their normal species values from Table 2.1. 
Table 2.3. Carbon substitution coordinates of iodobenzene.

\begin{tabular}{lcccrrr}
\hline \multicolumn{3}{c}{$\begin{array}{l}\text { Experiment } \\
\text { Carbon }\end{array}$} & $|a|(\AA)$ & $|b|(\AA)$ & $|c|(\AA)$ & \multicolumn{1}{c}{$a(\AA$ Initio } \\
\hline $\mathrm{C}_{1}$ & $0.734(3)$ & $0.05(4)$ & $0.087(27)$ & -0.776 & 0.000 & 0.000 \\
$\mathrm{C}_{2} / \mathrm{C}_{6}$ & $1.4469(13)$ & $1.2143(15)$ & {$[0]^{\mathrm{a}}$} & -1.466 & $+/-1.218$ & 0.000 \\
$\mathrm{C}_{3} / \mathrm{C}_{5}$ & $2.8468(8)$ & $1.2078(19)$ & {$[0]^{\mathrm{a}}$} & -2.865 & $+/-1.209$ & 0.000 \\
$\mathrm{C}_{4}$ & $3.5470(6)$ & $0.06(3)$ & $0.077(27)$ & 3.568 & 0.000 & 0.000 \\
\hline
\end{tabular}

${ }^{\mathrm{a}}$ Value constrained to zero. 
substitution coordinates of the carbon atoms of iodobenzene are compared to those from its $a b$ initio calculated structure. These coordinates were determined using the KRA program of Kisiel, with the error estimated by Costain's formula. ${ }^{23,24}$ It can be seen that carbons 1 and 4 have (small) determined non-zero values for $b$ and $c$; this is also reflected in the fact that the $A$ rotational constant decreases slightly in these isotopically substituted species, as seen in Table 2.2. This is attributed to zero-point vibrational effects, as the three lowest-frequency vibrational modes of iodobenzene (the iodine-phenyl in-plane and out-of-plane bends, as well as the C-I stretch) all have frequencies below $300 \mathrm{~cm}^{-1} \cdot{ }^{20}$ As the considerable errors that can arise in determining the coordinates of atoms near principal axes are well known, ${ }^{24,25}$ an effective least-squares fit of internal coordinates to the moments of inertia of iodobenzene and its isotopomers was also performed, using the STRFIT program of Kisiel; the results of this fit are presented in Table 2.4.

As discussed in a previous manuscript using this spectrometer, ${ }^{13}$ interference from wireless communication signals is present near $2.5 \mathrm{GHz}$, and so the spectrometer's performance is poor in this region. Attempts to isolate the detection electronics, particularly the low-noise, high-gain amplifier, by placing them inside the vacuum chamber, did not successfully eliminate this interference.

\section{b) CP-FTMW-MW double resonance spectroscopy}

Double resonance techniques that aid in spectral assignment are of tremendous utility in parsing dense rotational spectra, in which several species are present. The 
Table 2.4. Internal coordinates of iodobenzene, as determined by an effective leastsquares fit, compared to ab initio values. ${ }^{\text {a }}$

\begin{tabular}{lcc}
\hline Internal Coordinate & $r_{0}$ & Ab Initio \\
$r\left(\mathrm{C}_{2}-\mathrm{C}_{1}\right)(\AA)$ & $1.412(15)$ & 1.400 \\
$r\left(\mathrm{C}_{3}-\mathrm{C}_{2}\right)$ & $1.400(10)$ & 1.399 \\
$r\left(\mathrm{C}_{4}-\mathrm{C}_{3}\right)$ & $1.39(4)$ & 1.399 \\
$r\left(\mathrm{I}-\mathrm{C}_{1}\right)$ & $2.063(21)$ & 2.112 \\
$\angle\left(\mathrm{C}_{3}-\mathrm{C}_{2}-\mathrm{C}_{1}\right)(\mathrm{deg})$ & $119.9(10)$ & 119.2 \\
$\angle\left(\mathrm{C}_{2}-\mathrm{C}_{1}-\mathrm{C}_{6}\right)$ & $119.0(15)$ & 120.9 \\
$\angle\left(\mathrm{C}_{4}-\mathrm{C}_{3}-\mathrm{C}_{2}\right)$ & $120.5(12)$ & 120.5 \\
$\angle\left(\mathrm{I}-\mathrm{C}_{1}-\mathrm{C}_{2}\right)$ & $121.1(13)$ & 119.5
\end{tabular}

${ }^{a}$ All internal coordinates not listed in this Table were held fixed to their ab initio values. 
relative intensity accuracy and stability of the CP-FTMW spectrometer makes CPFTMW-MW spectroscopy a powerful tool. A $4 \mu \mathrm{s}$ single-frequency MW pulse, amplified by a 4 W SSA, following the chirped pulse destroys the rotational coherence of any rotational transition that shares a quantum state with the transition being pumped, reducing its intensity significantly. Transitions not connected to the pump transitions, meanwhile, are unaffected by the double resonance pulse. Using the CP-FTMW spectrometer for microwave-microwave double resonance spectroscopy allows all of the transitions that share a quantum state with the pump transition to be detected simultaneously.

In Figure 2.7, a demonstration of the CP-FTMW-MW double resonance technique is presented. The $J^{\prime}{ }_{K a^{\prime} K c^{\prime}} F^{\prime}-J^{\prime \prime}{ }_{K a^{\prime \prime} K c^{\prime \prime}} F^{\prime \prime}=4_{04} 11 / 2-3_{03} 9 / 2$ transition of iodobenzene is chosen as the double resonance pump transition. In the top panel, the fractional modulation of each rotational transition in the spectrum is shown. A total of six transitions are reduced in intensity by at least $50 \%$, and each of these transitions, and no others, share a quantum state with the pumped transition. Expanded spectra in the bottom two panels show that connected transitions are modulated selectively.

\section{c) Spectrum and structure of the iodobenzene-neon complex}

After the spectra of iodobenzene and its singly substituted ${ }^{13} \mathrm{C}$ isotopologues were removed from the 2-8 GHz CP-FTMW spectrum, a large number of transitions remained, showing a spacing pattern characteristic of an $a$-type spectrum with a single iodine 

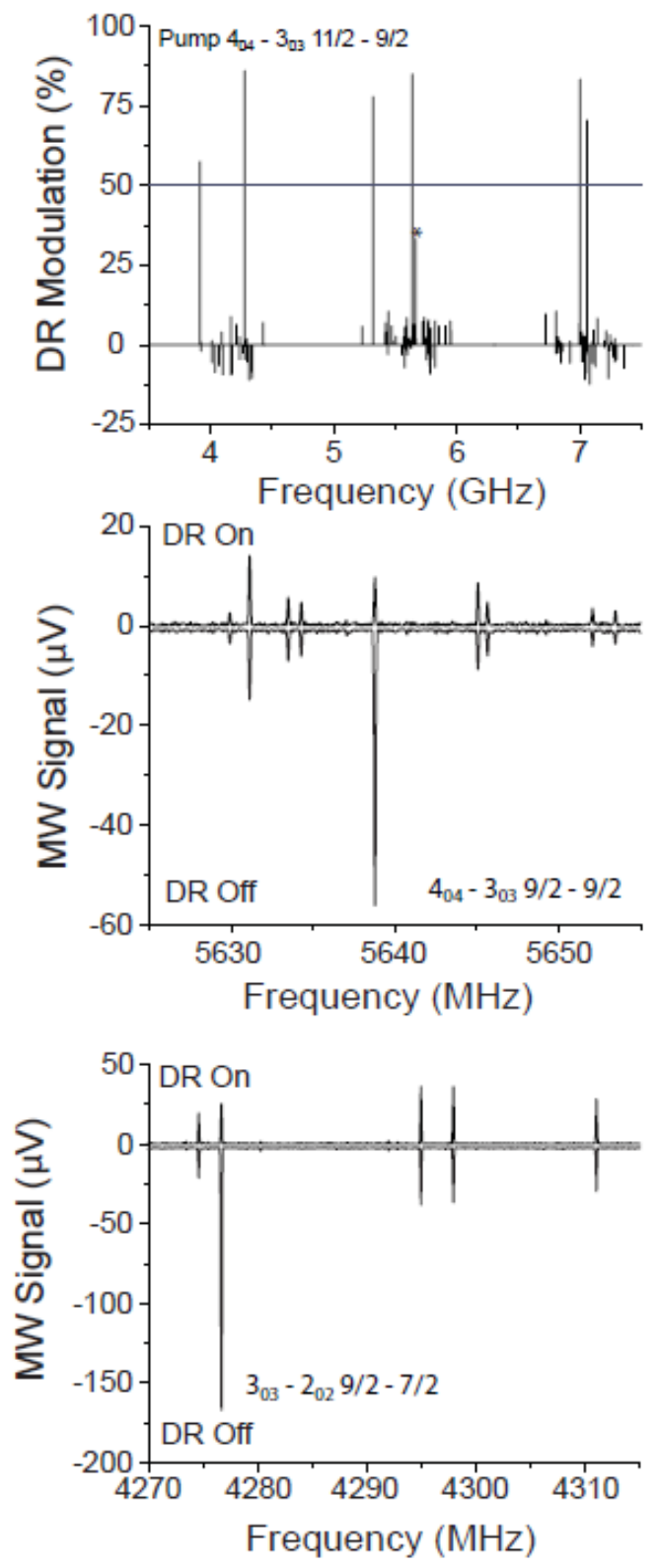

Figure 2.7. Demonstration of microwave-microwave double resonance spectroscopy on a 2-8 GHz CP-FTMW spectrometer. Top panel: fractional modulation of iodobenzene transitions upon excitation with a $4 \mathrm{~W}, 4 \mu \mathrm{s}$ single frequency pulse at the frequency of the $4_{04} 11 / 2-3_{03} 9 / 2$ transition. The asterisk indicates the pumped transition. The six transitions modulated by more than $50 \%$ are the only six transitions which share a quantum state with the pumped transition. Middle and bottom panel: expanded views comparing the spectra with the single-frequency pulse on and off, showing the selective modulation of connected transitions. 
nucleus present. From these transitions the spectrum of the complex of iodobenzene with a single neon atom was assigned. Two spectra, identified as the ${ }^{20} \mathrm{Ne}$ and ${ }^{22} \mathrm{Ne}$ isotopologues of the cluster, were fit. The fit Hamiltonian parameters of these two species are presented in Table 2.5 and compared to parameters from ab initio calculations.

The assignment of these two clusters as different isotopologues of the neoniodobenzene complex is confirmed by the nuclear quadrupole hyperfine tensors of the two species and a comparison to electronic structure calculations. In the bare iodobenzene molecule, the $a$ principal axis falls along the $\mathrm{I}_{-} \mathrm{C}_{1}$ bond; however, upon complexation with a neon atom, the principal axis system is rotated, and this bond is in the $a-b$ principal axis plane. By diagonalization of the quadrupole coupling tensor, as shown in Table 2.6, it can be seen that the eigenvalues of the quadrupole tensors of the two neon complexes differ from those of iodobenzene by less than $0.3 \%$, indicating that the electric field gradient at the iodine nucleus is nearly identical between the monomer and the $a$ principal axis is shown to be $\sim 10 \%$ larger in the ${ }^{22} \mathrm{Ne}$ complex than in the ${ }^{20} \mathrm{Ne}$ complex, in excellent agreement with ab initio calculations. If the weaker species were instead a vibrationally excited state of ${ }^{20} \mathrm{Ne}$-iodobenzene, this angle would be expected to be nearly the same between the two clusters.

In Figure 2.8 the isolated spectra of these two clusters are compared to simulations, using the fit Hamiltonian parameters in Table 2.5. It can be seen that the ${ }^{20} \mathrm{Ne}$-iodobenzene $/{ }^{22} \mathrm{Ne}$-iodobenzene signal intensity ratio is approximately 3.5:1, 
Table 2.5. Fit A-reduction Hamiltonian parameters of the neon-iodobenzene complex.

\begin{tabular}{|c|c|c|c|c|}
\hline \multirow[b]{2}{*}{ Parameter } & \multicolumn{2}{|c|}{ Iodobenzene $-{ }^{20} \mathrm{Ne}$} & \multicolumn{2}{|c|}{ Iodobenzene $-{ }^{22} \mathrm{Ne}$} \\
\hline & Experiment & Ab Initio & Experiment & Ab Initio \\
\hline$A(\mathrm{MHz})$ & 1818.674(20) & 2005.59 & $1731.58(4)$ & 1911.08 \\
\hline$B(\mathrm{MHz})$ & $605.6455(12)$ & 592.13 & $598.7810(16)$ & 585.51 \\
\hline$C(\mathrm{MHz})$ & $537.5551(11)$ & 545.38 & $524.3010(12)$ & 532.67 \\
\hline$\Delta_{J}(\mathrm{kHz})$ & $1.192(7)$ & & $1.342(9)$ & \\
\hline$\Delta_{J K}(\mathrm{kHz})$ & $18.30(4)$ & & $15.68(7)$ & \\
\hline$\Delta_{K}(\mathrm{kHz})$ & $16.7(28)$ & & $10(5)$ & \\
\hline$\delta_{J}(\mathrm{kHz})$ & $0.294(5)$ & & $0.356(8)$ & \\
\hline$\delta_{K}(\mathrm{kHz})$ & $13.0(3)$ & & $12.7(4)$ & \\
\hline$\chi_{a a}(\mathrm{MHz})$ & $-1779.38(4)$ & -1711.36 & $-1755.14(8)$ & -1692.84 \\
\hline$\chi_{b b}-\chi_{c c}(\mathrm{MHz})$ & $-177.06(17)$ & -135.72 & $-201.28(22)$ & -154.24 \\
\hline$\chi_{a b}(\mathrm{MHz})$ & $544.26(6)$ & 483.89 & 598.24(11) & 529.23 \\
\hline No of lines & 123 & & 72 & \\
\hline $\begin{array}{l}\text { rms error } \\
(\mathrm{kHz})\end{array}$ & 9.8 & & 7.8 & \\
\hline
\end{tabular}


Table 2.6. Principal nuclear quadrupole coupling tensors of iodobenzene and its neon complexes.

\begin{tabular}{lrcc}
\hline Parameter & Iodobenzene & Iodobenzene $-{ }^{20} \mathrm{Ne}$ & Iodobenzene- ${ }^{22} \mathrm{Ne}$ \\
\hline$\chi_{z z}(\mathrm{MHz})^{\mathrm{a}}$ & $-1892.039(5)$ & $-1889.48(5)$ & $-1889.38(8)$ \\
$\chi_{x x}(\mathrm{MHz})$ & $978.816(6)$ & $978.22(9)$ & $978.21(10)$ \\
$\chi_{y y}(\mathrm{MHz})$ & $913.222(6)$ & $911.25(9)$ & $911.16(10)$ \\
$\theta_{a z}(\mathrm{deg})$ expt. & 0 & $11.4355(14)$ & $12.6461(19)$ \\
$\theta_{a z}(\mathrm{deg}) a b$ initio & 0 & 10.531 & 11.578 \\
\hline
\end{tabular}

${ }^{\mathrm{a}}$ The $z$-axis is collinear with the C-I bond, and the $a$ principal axis of iodobenzene, and the $x$-axis is in the plane with the benzene ring, the $b$ principal axis of iodobenzene. 

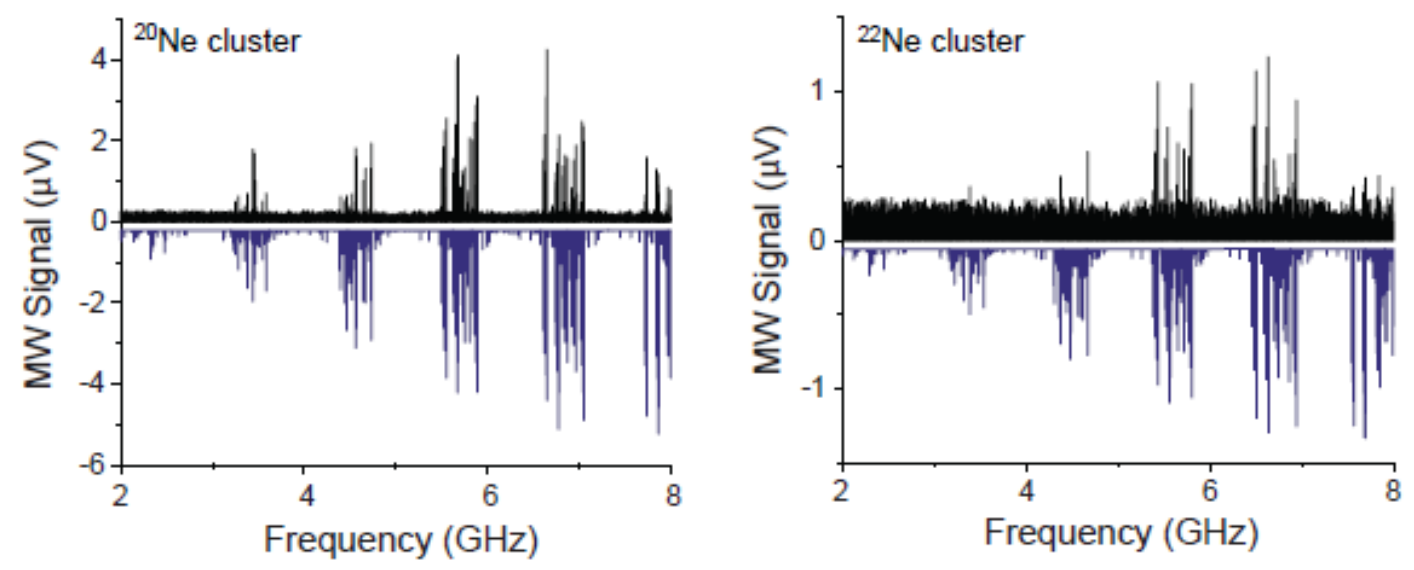

Figure 2.8. Isolated rotational spectra of the two assigned isotopic species of the iodobenzene- neon complex, compared to simulated spectra with fit Hamiltonian parameters. An intensity rolloff is observed above $7.5 \mathrm{GHz}$ due to lower TWTA power output and reduced horn transmission efficiency. 
compared to the natural relative abundance ratio of 9.8:1. This anomalous relative intensity in these isotopes of neon in weakly bound clusters has been previously observed and commented on by several research groups. It has been attributed to the slightly lower zero-point vibrational energy, and hence higher dissociation energy, of clusters with the heavier ${ }^{22} \mathrm{Ne}$ isotope, leading to preferential stabilization of these clusters in the supersonic expansion. ${ }^{26-28}$

The structure of this cluster can be determined using the method described by Suenram et al. for the argon-formamide cluster $^{29}$ and implemented in the RGDFIT program. ${ }^{30}$ The structure of the iodobenzene molecule is assumed to be unchanged upon complexation, and so there are only three variable structural parameters in the structure of the iodobenzene - neon cluster, defining the position of the neon atom. These parameters are presented in Table 2.7 for the ${ }^{20} \mathrm{Ne}$ and ${ }^{22} \mathrm{Ne}$ clusters. In Table 2.8 , the angle between the $a$ principal axis of the iodobenzene-neon cluster and the I-C bond as derived by diagonalization of the quadrupole coupling tensor is compared to that of this derived structure. The two methods for determining this angle yield very similar results (within $3 \%)$

This analysis, however, does not determine the sign of the coordinates of the neon atom, and so it could be located in any octant; due to the symmetry of iodobenzene, this results in two possible structures for the complex, which are presented in panel (A) of Figure 2.9. In structure (a), the neon atom is located over the phenyl ring, while in structure (b), it is located above the iodine atom. Both structures accurately reproduce 
Table 2.7. Experimentally derived geometry of the iodobenzene-neon complex.

\begin{tabular}{lllllll}
\hline Species & \multicolumn{3}{c}{ Spherical Coordinates } & \multicolumn{3}{c}{ Cartesian Coordinates $^{\mathrm{b}}$} \\
& $R$ & $\theta$ & $\varphi$ & $|z|$ & $|x|$ & $|y|$ \\
\hline & & & & & & \\
Iodobenzene $-{ }^{20} \mathrm{Ne}$ & 3.805 & 27.93 & 15.27 & 1.719 & 0.469 & 3.362 \\
Iodobenzene- ${ }^{22} \mathrm{Ne}$ & 3.804 & 28.12 & 15.90 & 1.724 & 0.491 & 3.355
\end{tabular}

${ }^{\mathrm{a}} R$ is the distance (in angstroms) between the neon atom and the center of mass of iodobenzene; $\theta$ is the angle between a vector along $R$ and the $c$ principal axis of iodobenzene, and $\varphi$ is the angle between the projection of a vector along $R$ into the $a-b$ principal axis plane and the $a$ principal axis of iodobenzene.

${ }^{\mathrm{b}}$ Coordinates (in angstroms) in the principal axis system of the iodobenzene monomer, with $z$ as the $a$ axis, $x$ as the $b$ axis, and $y$ as the $c$ axis.

Table 2.8. Angle (in degrees) between the $a$ principal axis of the iodobenzene-neon cluster and the $\mathrm{I}-\mathrm{C}_{1}$ bond.

\begin{tabular}{lcc}
\hline Method & Iodobenzene- ${ }^{20} \mathrm{Ne}$ & Iodobenzene- ${ }^{22} \mathrm{Ne}$ \\
\hline Quadrupole tensor & $11.4355(14)$ & $12.6461(19)$ \\
Structure fit & 11.173 & 12.372 \\
Ab initio & 10.531 & 11.578 \\
\hline
\end{tabular}


(A) Iodobenzene-neon

Structure (a)
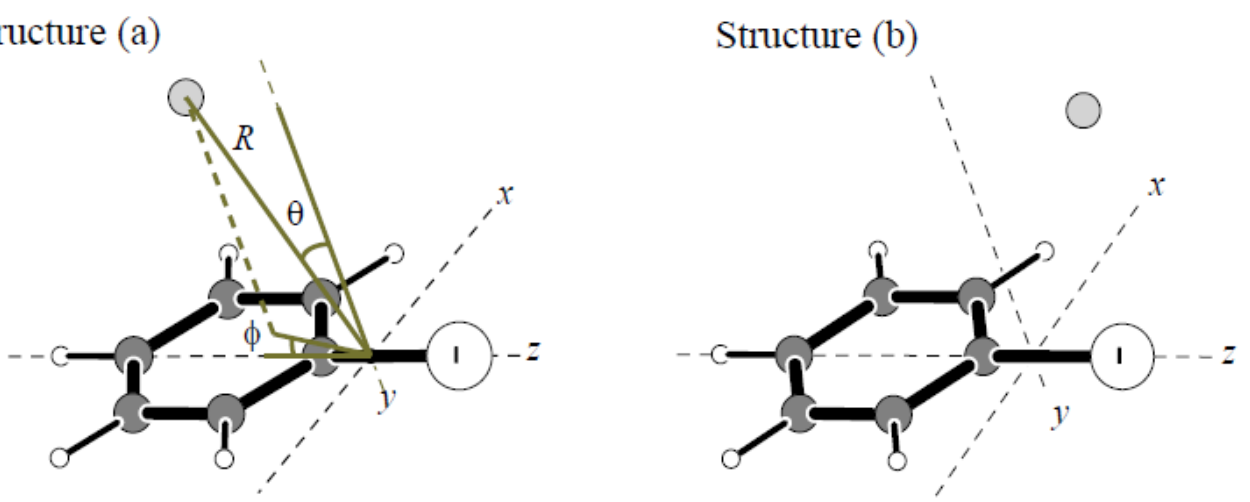

(B) Fluorobenzene-neon

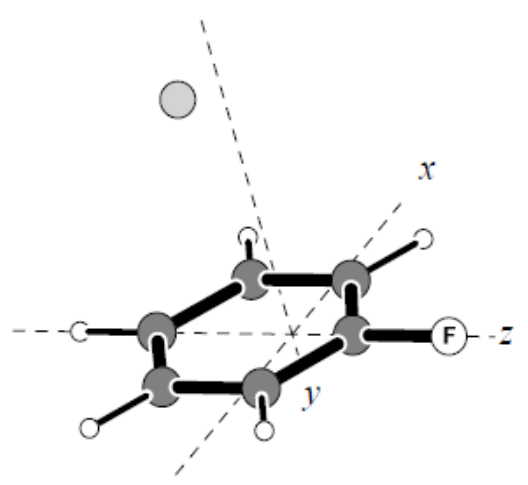

(C) Chlorobenzene-neon

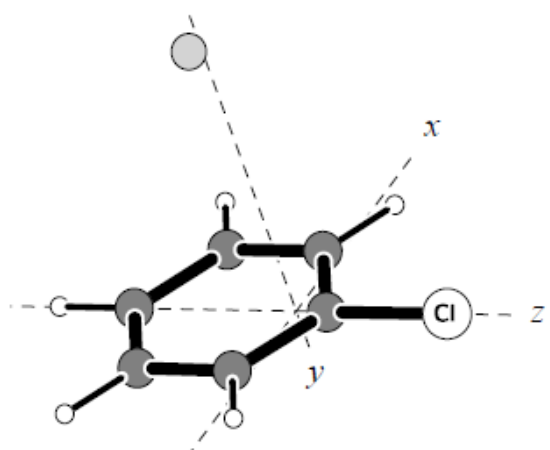

Figure 2.9. Experimentally derived structures of halobenzene-neon complexes. Panel (A) shows the two possible structures of the iodobenzene-neon complex as derived from the structural analysis in the text. In panels (B) and (C) the structures of fluorobenzene-neon and chlorobenzene-neon, from previous studies by microwave spectroscopy, ${ }^{31,32}$ are shown. The structural parameters $R, \theta$, and $\varphi$ from Table 2.7 are illustrated. The $x, y$, and $z$ axes signify the $a, b$, and $c$ principal axes, respectively, of the halobenzene monomer. 
the observed rotational constants of the iodobenzene-neon cluster. However, only structure (a) corresponds to an ab initio optimized structure; structure (b) minimizes to a different structure upon geometry optimization. In addition, structure (a) corresponds closely to the experimentally derived structures of the fluorobenzene- neon ${ }^{31}$ and chlorobenzene - neon ${ }^{32}$ complexes, which are shown in panels (B) and (C) of Figure 2.9. The structures of these complexes were confirmed through isotopic substitution measurements in other positions of the molecule. Additionally, the van der Waals radii of iodine and neon serve as further confirmation of the assignment of the iodobenzeneneon complex as structure (a). From the tabulations of Bondi, ${ }^{33}$ the sum of the radii of iodine and neon is $3.52 \AA$, while in structure (b), the I-Ne distance is only $3.42 \AA$, which is quite unlikely for a van der Waals complex.

As seen in Table 2.7, in the RGDFIT-derived structure of iodobenzene, the neon atom is not located in the $a-c$ symmetry plane of iodobenzene, instead having an $x$ coordinate of $0.46 \AA$ for the ${ }^{20} \mathrm{Ne}$ complex, and $0.49 \AA$ for the ${ }^{22} \mathrm{Ne}$ complex. A similar effect is also observed in the chlorobenzene-neon and fluorobenzene-neon clusters, and is due to the large-amplitude zero-point motions of these floppy clusters, making a precise structure determination difficult. $^{34}$

\section{Conclusion}

In this chapter, the design and performance of a direct digital 2-8 GHz chirpedpulse FTMW spectrometer is discussed. A measurement of the rotational spectrum of iodobenzene demonstrates the sensitivity and relative intensity accuracy of this 
spectrometer, and from this spectrum the structure of iodobenzene and its neon complex is derived. In addition, a method for performing microwave-microwave double resonance experiments is presented. This spectrometer design has favorable characteristics for the study of the structure and dynamics of large molecules and clusters. 


\section{Chapter 2 References}

(1) This chapter contains material from a published manuscript: Neill, J.L.; Shipman, S.T.; Alvarez-Valtierra, L.; Lesarri, A.; Kisiel, Z.; Pate, B.H. J. Mol. Spectrosc., in press.

(2) Brenner, V.; Piuzzi, F.; Dimicoli, I.; Tardivel, B.; Mons, M. J. Phys. Chem. A 2007, 111, 7347-7354.

(3) Cocinero, E. J.; Stanca-Kaposta, E. C.; Gamblin, D. P.; Davis, B. G.; Simons, J. P. J. Am. Chem. Soc. 2008, 131, 1282-1287.

(4) Stearns, J. A.; Seaiby, C.; Boyarkin, O. V.; Rizzo, T. R. Phys. Chem. Chem. Phys. 2009, 11, 125-132.

(5) Alvarez-Valtierra, L.; Yi, J. T.; Pratt, D. W. J. Phys. Chem. A 2009, 113, 22612267.

(6) Alonso, J. L.; Sanz, M. E.; Lopez, J. C.; Cortijo, V. J. Am. Chem. Soc. 2009, 131, $4320-4326$.

(7) Riveros, J. M.; Wilson, J. E. B. J. Chem. Phys. 1967, 46, 4605-4612.

(8) I.R. Medvedev, F. C. D. L., E. Herbst. Astrophys. J. Suppl. Ser. 2009, 181, 433.

(9) Sanchez, R.; Caminati, W.; López, J. C.; Alonso, J. L. Chem. Phys. Lett. 2005, $414,226-229$.

(10) Sarkozy, L.; Kukolich, S. G. Construction and Development of a New LowFrequency Fourier Transform Spectrometer for Operation in the 1-6 GHz Range. 65th International Symposium on Molecular Spectroscopy Columbus, OH, 2010, TC06. 
(11) Storm, V.; Dreizler, H.; Consalvo, D.; Grabow, J. U.; Merke, I. Rev. Sci. Instrum. 1996, 67, 2714-2719.

(12) Etchison, K. C.; Dewberry, C. T.; Kerr, K. E.; Shoup, D. W.; Cooke, S. A. J. Mol. Spectrosc. 2007, 242, 39-45.

(13) Kisiel, Z.; Krasnicki, A.; Pszczólkowski, L.; Shipman, S. T.; Alvarez-Valtierra, L.; Pate, B. H. J. Mol. Spectrosc. 2009, 257, 177-186.

(14) Elliot, D. F. Handbook of Digital Signal Processing Engineering Applications; Academic: San Diego, 1987.

(15) Frisch, M. J. T., G. W.; Schlegel, H. B.; Scuseria, G. E.;; Robb, M. A. C., J. R.; Montgomery, J. A., Jr.; Vreven, T.;; Kudin, K. N. B., J. C.; Millam, J. M.; Iyengar, S. S.; Tomasi, J.;; Barone, V. M., B.; Cossi, M.; Scalmani, G.; Rega, N.; Petersson,; G. A.; Nakatsuji, H. H., M.; Ehara, M.; Toyota, K.; Fukuda, R.;; Hasegawa, J. I., M.; Nakajima, T.; Honda, Y.; Kitao, O.; Nakai, H.;; Klene, M. L., X.; Knox, J. E.; Hratchian, H. P.; Cross, J. B.; Bakken, V.;; Adamo, C. J., J.; Gomperts, R.; Stratmann, R. E.; Yazyev, O.;; Austin, A. J. C., R.; Pomelli, C.; Ochterski, J. W.; Ayala, P. Y.;; Morokuma, K. V., G. A.; Salvador, P.; Dannenberg, J. J.; Zakrzewski,; V. G.; Dapprich, S. D., A. D.; Strain, M. C.; Farkas, O.; Malick, D.; K.; Rabuck, A. D. R., K.; Foresman, J. B.; Ortiz, J. V.; Cui, Q.;; Baboul, A. G. C., S.; Cioslowski, J.; Stefanov, B. B.; Liu, G.;; Liashenko, A. P., P.; Komaromi, I.; Martin, R. L.; Fox, D. J.; Keith,; T.; AlLaham, M. A. P., C. Y.; Nanayakkara, A.; Challacombe, M.;; Gill, P. M. W. J., 
B.; Chen, W.; Wong, M. W.; Gonzalez, C.;; Pople, J. A. Gaussian 03, revision E.01; Gaussian, Inc.: Wallingford, CT, 2004.

(16) Feller, D. J. Comput. Chem. 1996, 17, 1571-1586.

(17) Schuchardt, K. L.; Didier, B. T.; Elsethagen, T.; Sun, L.; Gurumoorthi, V.; Chase, J.; Li, J.; Windus, T. L. J. Chem. Inf. Model. 2007, 47, 1045-1052.

(18) Glukhovtsev, M. N.; Pross, A.; McGrath, M. P.; Radom, L. J. Chem. Phys. 1995, $103,1878-1885$.

(19) Mirri, A. M.; Caminati, W. Chem. Phys. Lett. 1971, 8, 409-412.

(20) Dorosh, O.; Bialkowska-Jaworska, E.; Kisiel, Z.; Pszczólkowski, L. J. Mol. Spectrosc. 2007, 246, 228-232.

(21) Pickett, H. M. J. Mol. Spectrosc. 1991, 148, 371-377.

(22) Kisiel, Z. PROSPE, Programs for ROtational SPEctroscopy, http://info.ifpan.edu.pl/ kisiel/prospe.htm.

(23) Costain, C. C. Trans. Am. Cryst. Assoc. 1966, 2, 157-164.

(24) Demaison, J.; Rudolph, H. D. J. Mol. Spectrosc. 2002, 215, 78-84.

(25) Watson, J. K. G.; Roytburg, A.; Ulrich, W. J. Mol. Spectrosc. 1999, 196, 102-119.

(26) Brookes, M. D.; Hughes, D. J.; Howard, B. J. J. Chem. Phys. 1996, 104, 53915405.

(27) Xu, Y.; Jager, W. J. Chem. Phys. 1997, 107, 4788-4796.

(28) Xu, Y.; Jager, W. Phys. Chem. Chem. Phys. 2000, 2, 3549-3553.

(29) Suenram, R. D.; Fraser, G. T.; Lovas, F. J.; Gillies, C. W.; Zozom, J. J. Chem. Phys. 1988, 89, 6141-6146. 
(30) Kisiel, Z.; Fowler, P. W.; Legon, A. C. J. Chem. Phys. 1991, 95, 2283-2291.

(31) Wilson, R. J.; Peebles, S. A.; Antolinez, S.; Sanz, M. E.; Kuczkowski, R. L. J. Phys. Chem. A 1998, 102, 10630-10635.

(32) Oh, J.-J.; Park, I.; Peebles, S. A.; Kuczkowski, R. L. J. Mol. Struct. 2001, 599, 1522.

(33) Bondi, A. J. Phys. Chem. 1964, 68, 441-451.

(34) Brupbacher, T.; Makarewicz, J.; Bauder, A. J. Chem. Phys. 1994, 101, 97369746. 


\section{Chapter 3}

\section{Application of High-Sensitivity CP-FTMW Spectroscopy to the Structural Determination of Large Molecules and Clusters}

\section{Introduction}

In the previous chapter, the application of CP-FTMW spectroscopy in the 2-8 $\mathrm{GHz}$ frequency range to the rotational spectrum of iodobenzene was described. This study illustrated the potential of CP-FTMW spectroscopy to provide accurate structural information for large molecules through the measurement of isotopically labeled species in natural abundance. Additionally, the spectrum of the complex of iodobenzene with a neon atom was assigned for the first time, from which structural information about this complex could be extracted. These new assignments were possible because of the high sensitivity of the spectrum, which in this case was achieved by using two pulsed nozzles and by acquiring a large number of FIDs $(210,000)$.

In the last few years, we have implemented "deep averaging” (defined here as at least 100,000 FID averages) as a routine aspect of CP-FTMW spectroscopy. In most of the CP-FTMW studies that have been performed in our lab in the past few years, the spectral assignments that are of the most interest are those that are of weak lines (typically 10:1 signal to noise or less), whether they are minor isotopologues in order to derive structural information, conformational isomers with low populations, or weakly bound complexes. The two modifications to the spectrometer that have made these deep averaging studies feasible are the construction of a new vacuum chamber that can incorporate three pulsed nozzles to increase the number of molecules probed on each 
valve injection cycle, and a new digital oscilloscope that is very clean (i.e., has very few spurious signals), can effectively acquire multiple FID traces on each valve pulse, and acquires spectra considerably faster than the previous generation of oscilloscopes. These advances have been primarily implemented in the $7.0-18.5 \mathrm{GHz}$ frequency range to date, and this spectrometer will be described in this chapter in detail, with an emphasis on operating procedures that are different than in the previously published spectrometer design. For larger systems, reduced-bandwidth implementations will be described, which are sometimes desirable because, despite the advances of the past few years, the repetition rate of the spectrometer is still limited by oscilloscope throughput.

To accompany the development of high-sensitivity CP-FTMW spectroscopy, complementary techniques to aid in spectral assignment and structural determination are needed. For spectral assignment, two approaches have been implemented. The first is microwave-microwave double resonance spectroscopy, which was described in Chapter 2 in a CP-FTMW spectrometer. It can also be performed in a Balle-Flygare-type cavity spectrometer as technique for extracting the quantum state connections between observed transitions. Secondly, the development and performance of an automated fitting program will be described, which serves as an efficient way to screen for proposed structures. This is of particular utility in the assignment of isotopologues, which can be extremely challenging due to their weak signal levels, particularly when the structure of the complex is not known in advance.

The dipole moment of a species, as will be demonstrated below, is often a very important secondary parameter for comparison of calculated and experimental structures, 
as for large systems is it often common for many possible isomers to have similar structures. The orientation of the dipole moment in the principal axis system can be determined from the relative intensities of $a, b$, and $c$-type transitions; however, its magnitude cannot be found through this method. Stark effect measurements in a CPFTMW spectrometer are highly efficient due to the potential to measure all transitions of all species in a gas mixture, along with a calibrant gas, in a single measurement, from which the permanent dipole moments of all of these components can be derived. The combination of direct structural information and the molecular dipole moment enables confident structural assignments, even for large, floppy molecules and clusters.

\section{Spectrometer Design and Techniques for Spectral Analysis}

a) High-sensitivity 7.0-18.5 GHz CP-FTMW spectroscopy

A schematic of the high-sensitivity 7.0-18.5 GHz CP-FTMW spectrometer is presented in Figure 3.1. A chirped pulse, typically $1 \mu$ s in duration, from $12.0-0.5 \mathrm{GHz}$ is generated by a $24 \mathrm{GS} / \mathrm{s}$ sampling rate AWG (Tektronix AWG7122B, with interleave option). The output of the AWG is filtered by an eight-pole, $12.2 \mathrm{GHz}$ low-pass filter (K\&L Microwave). Unlike in the 2-8 GHz spectrometer, where the AWG-generated chirped pulse could be input directly into a traveling wave tube amplifier (TWTA), a frequency upconversion step must be employed because AWG technology cannot currently generate pulses directly in the $7.0-18.5 \mathrm{GHz}$ region. Therefore, the pulse is upconverted with an $18.95 \mathrm{GHz}$ phase-locked dielectric resonant oscillator (PDRO) in a 


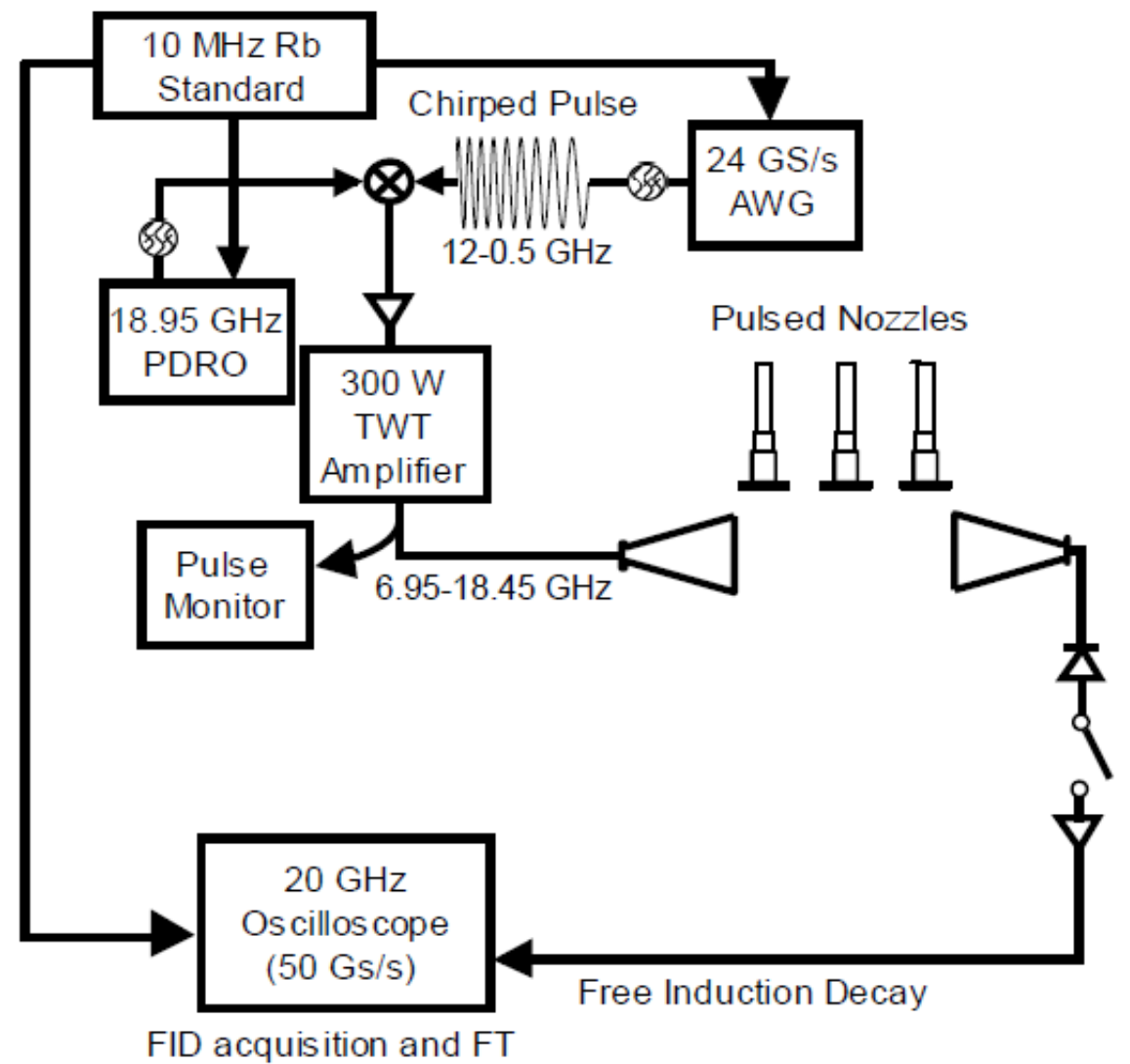

Figure 3.1. Schematic of the 7.0-18.5 GHz CP-FTMW spectrometer for multi-nozzle, multi-FID operation. 
triple-balanced microwave mixer (Miteq, TB0440LW1). The output of the PDRO is spectrally purified by a cavity bandpass filter with $200 \mathrm{MHz}$ bandwidth (Lorch Microwave). This yields a $6.95-18.45 \mathrm{GHz}$ chirped pulse. The microwave mixer is double-sideband, so a second sweep from $30.95-19.45 \mathrm{GHz}$ is also generated. This sideband is not actively filtered out because it is out of the bandwidth of subsequent components; it is has not been observed to cause any problems in the spectrometer to date.

The desired power input level to the TWTA for maximum output power is $\sim 1$ $\mathrm{mW}(0 \mathrm{dBm})$. The output power of the AWG is $1 \mathrm{~mW}$, but the conversion loss of the mixer $(\sim 10 \mathrm{~dB})$ reduces its power level to $\sim 0.1 \mathrm{~mW}(-10 \mathrm{dBm})$ in the upconversion step. Therefore, we employ a general-purpose amplifier (Wright Technologies, formerly Phase One Microwave, SL18-2015, 6-18 GHz, $20 \mathrm{~dB}$ gain). Following this amplifier, an attenuator is used to reduce the power level from $10 \mathrm{~mW}(+10 \mathrm{dBm})$ post-amplifier back to $1 \mathrm{~mW}$. The TWTAs in our laboratory are rated for input power levels of up to $10 \mathrm{~mW}$, but higher power levels could damage the amplifier and so we attenuate the pulse power to provide additional insurance against this possibility.

Two TWTAs in this frequency range are currently in our laboratory, both manufactured by Amplifier Research: one with $1 \mathrm{~kW}$ output power (1000TP8G18) and the other with $200 \mathrm{~W}$ output power (200T8G18A). Both amplifiers are pulsed with TTL control, which is essential to eliminate noise contributions from the amplifier during FID detection. Currently, we use the $200 \mathrm{~W}$ almost exclusively in the lab, which has been found to be necessary for the detection of multiple FID traces per valve pulse, because 
the passive diode limiter used on the receiver cannot survive multiple pulses in such a short time frame with the $1 \mathrm{~kW}$ amplifier. Both amplifiers have WRD-750 double-ridged waveguide outputs.

The microwave horn antennas used from 7.0-18.5 GHz (Advanced Technical Materials, 20.5-24 dB gain) are mounted onto bulkhead feedthroughs (Advanced Technical Materials) to couple microwave radiation into and out of the vacuum chamber. The feedthroughs are attached to custom-drilled ISO-200 quick flanges with bolt holes to match the bolt circle of the feedthrough, and a groove for the addition of an O-ring to create a vacuum seal. A pressure-sealing Kapton window is attached between the horn and the bulkhead feedthrough. Having the horns mounted directly on bulkhead feedthroughs is advantageous, because at these frequencies, waveguide has far lower loss per foot than coaxial cable, and therefore the minimization of coaxial connections, which can attenuate the power of the TWTA pulse and the molecular FIDs, is valuable. This also makes horn alignment far simpler than coupling into the vacuum chamber on coaxial cable and mounting the horns inside the chamber. No components to hold the horn inside the vacuum chamber are needed.

The horn antennas are separated by approximately $60 \mathrm{~cm}$ (horn tip to horn tip), allowing for the accommodation of three pulsed nozzles, oriented perpendicular to the axis of microwave propagation. The nozzles are separated by $20 \mathrm{~cm}$ so as to avoid interference between the expansions. As in the $2-8 \mathrm{GHz}$ spectrometer design, these are $0.9 \mathrm{~mm}$ diameter General Valve Series 9 nozzles. For both of the studies described in this chapter, nozzles modified to include a reservoir to contain liquid or solid samples ${ }^{1}$ 
were used. These nozzles are mounted in a copper bar, which is resistively heated, and its temperature monitored by a thermistor. The nozzles are typically slightly above the top of the horn antenna. The receiver is nearly identical to that described in Chapter 2. The solid state PIN diode limiter and SPST switch are the same part numbers as in that design, while the amplifier is replaced by a similar one at higher frequency (AFS5-08001800-14-10P-5, $38 \mathrm{~dB}$ gain, $1.4 \mathrm{~dB}$ noise figure). The amplified molecular FID signals are fed into a $50 \mathrm{GS} / \mathrm{s}$ oscilloscope with $16 \mathrm{GHz}$ hardware bandwidth (Tektronix DPO72004), and are typically digitized for either 10 or $20 \mu$ s per FID, depending on the carrier gas used.

For most applications, 10 microwave pulses, separated by approximately $30 \mu \mathrm{s}$ in order to give molecular emissions adequate time to dephase between excitation pulses, are applied, and 10 molecular FID signals collected. The passive diode limiter is important for this application; its rated power handling capacity is $1 \mathrm{~kW}$ for $1 \mu \mathrm{s}$, but as mentioned above, we have observed that in the multi-FID mode, the diode limiter does not fully recover between pulses, and so with the $1 \mathrm{~kW}$ TWTA, diode limiters are frequently blown. With the $200 \mathrm{~W}$ TWTA, damage is extremely infrequent. It should be noted that the excitation pulse length is typically $1 \mu \mathrm{s}$, which is also to limit the likelihood of component damage. With typical FID dephasing times of $\sim 10 \mu \mathrm{s}$ in this frequency range, longer excitation pulses may sometimes be desirable, particularly for molecules with low dipole moments; the excitation pulse has been lengthened to $2 \mu$ s for a few applications without damage, but this operation mode has not been tested extensively. 
The oscilloscope is operated in Fast Frame mode, in which the oscilloscope memory is split into 10 segments. The oscilloscope is triggered before each molecular FID detection, and on each valve injection cycle a FID is added into each segment. The frames are then added into an average frame, which for single-resonance applications is all that is desired. The nozzle repetition rate, as mentioned above, is limited by the data processing throughput of the oscilloscope; for $20 \mu$ s FID acquisition $\left(10^{7}\right.$ points acquired by the oscilloscope per valve injection cycle), the nozzles are run at $0.67 \mathrm{~Hz}$. This leads to an acquisition rate of 24,000 FIDs per hour of signal averaging. As will be described in future applications, it is anticipated that this repetition rate will increase in the near future, reducing the time required to acquire a deep-average CP-FTMW spectrum. Spectra are typically acquired while no one is present in the lab; in this operation mode, a spectrum is saved automatically every $\sim 2$ hours to limit the impact of power outages due to weather or other factors, phase shifts that can occasionally occur for various reasons, or deterioration in the signal level. In a typical "overnight" scan $(\sim 14 \mathrm{~h})$, approximately 350,000 signal averages are obtained. When needed, spectra can be run for multiple days to increase sensitivity further.

The co-added FID spectrum is Fourier transformed to yield the frequency spectrum. A digital frequency filter is applied before Fourier transformation. This is crucial to suppress the side lobes that result from the fact that the FID amplitude is highest at the beginning of the spectrum, resulting in a discontinuity. There are three windows that are most typically used. The most common is the Kaiser-Bessel window, which has the advantage of extremely good sidelobe suppression $(69 \mathrm{~dB}$, or a factor of 
$\sim 3,000$ in electric field strength), but the disadvantage of broadening the full-width at half-maximum (FWHM) linewidth by 93\%. The Hamming window is not as good for sidelobe suppression ( $43 \mathrm{~dB}$, or a factor of 140 ) but does not broaden the linewidth as much $(50 \%)$. For cases where minimum linewidth is crucial (particularly in the case of small splittings), resolution enhancement filters of the type used in NMR can be applied. ${ }^{2}$ The filter involves multiplying the FID by an increasing exponential that corresponds to the $T_{2}$ dephasing time of the molecular transitions, followed by the application of a Gaussian. This window has a far lower FWHM linewidth than either the Kaiser-Bessel or Hamming windows, but the signal-to-noise ratio is reduced and considerable side lobes remain. For most applications, particularly in the study of dense spectra, baseline resolution is the most important factor, so that weak lines that lie very near in frequency to strong lines can be seen; therefore the Kaiser-Bessel window is most desirable. However, when transitions have structure due to internal motion or hyperfine splitting, this can be smeared by this window. As the window is applied in post-processing, spectra with multiple window functions can be obtained from the same data set, and the complementary strengths of these windows can be exploited.

\section{b) Reduced-bandwidth CP-FTMW spectroscopy for deeper averaging}

Since the report of the original 7.0-18.5 GHz CP-FTMW spectrometer, several reduced-bandwidth configurations of this spectrometer have been described. ${ }^{3-6}$ These offer the advantage of significantly reducing the cost of the spectrometer; the AWG and oscilloscope can both be replaced with lower-bandwidth components, while a lower- 
power amplifier can be used. Because the data record length is significantly reduced from the full-bandwidth spectrometer due to the reduced digitization rate, this spectrometer design offers advantages even with the high-bandwidth equipment in our laboratory; because the repetition rate is data throughput-limited, the spectrometer can reach a given sensitivity level faster in this configuration.

This configuration is particularly appealing when studying larger species; these molecules have higher spectral line density, and therefore it is common that a few $\mathrm{GHz}$ of spectral acquisition at high sensitivity is sufficient for spectral characterization. This spectrometer uses a heterodyne receiver instead of the direct digitization approach described above. A schematic of the spectrometer in this configuration is presented in Figure 3.2, demonstrated for the 7.0-8.8 GHz spectral range. The pulse generation circuit, in this case, is simplified by the fact that this bandwidth can be addressed directly by the AWG, and so the $18.95 \mathrm{GHz}$ PDRO is removed from the system, and is therefore identical to that described in Chapter 2.

The receiver consists of the same three components (passive diode limiter, SPST switch, low-noise amplifier) as above, followed by a bandpass filter for the desired bandwidth (13IZ4-7900/1850-S, Lorch Microwave). This is needed to eliminate the contamination of both noise and spectral lines outside of the desired bandwidth, which would alias into the measured frequency range, increasing the noise floor. The molecular signals are then mixed with a $9.9 \mathrm{GHz}$ PDRO in a double-balanced mixer (Miteq, 


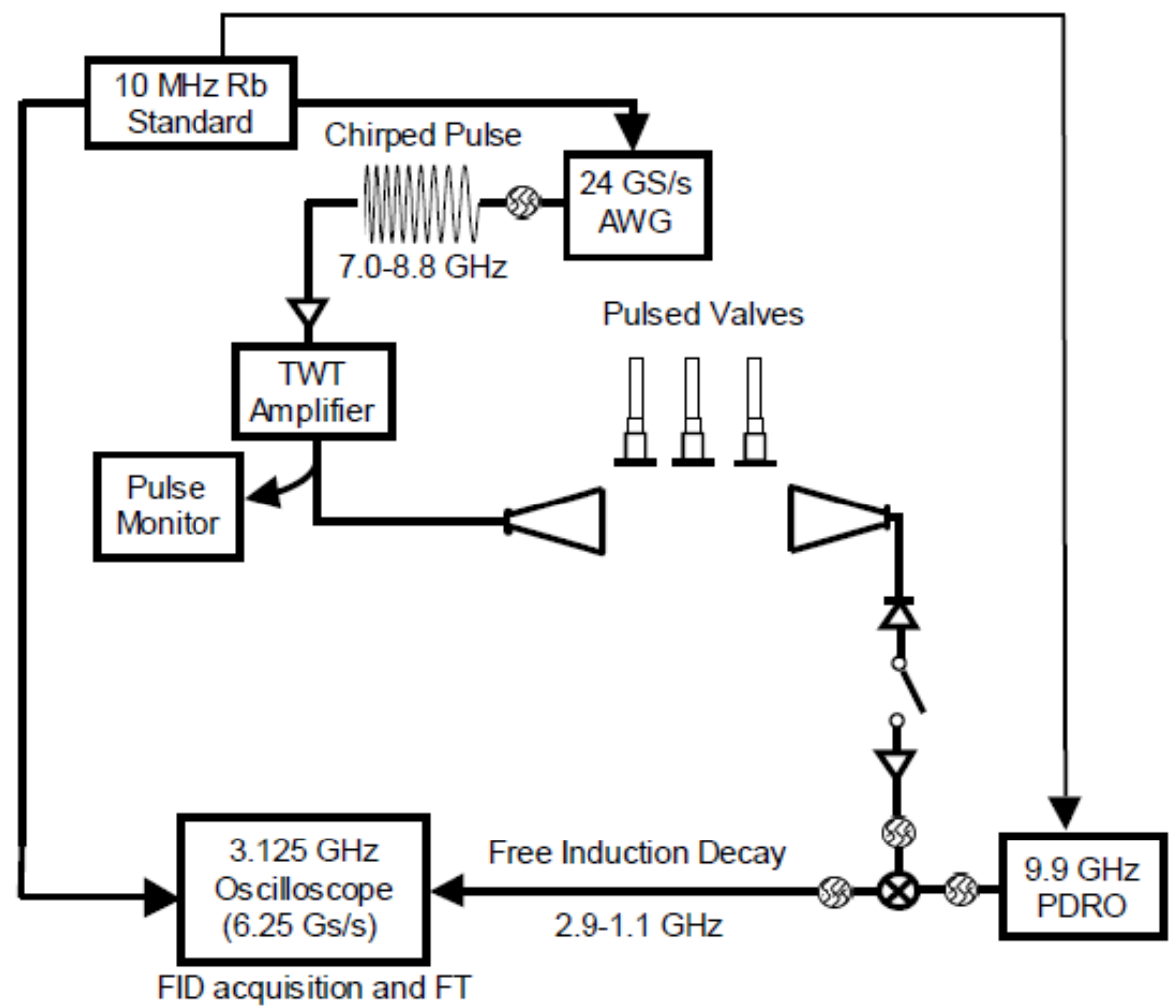

Figure 3.2. Schematic of the reduced-bandwidth 7.0-8.8 GHz CP-FTMW spectrometer to increase the rate of signal averaging. 
DM0520LW1); the output of this PDRO has been filtered as for the $18.95 \mathrm{GHz}$ PDRO. The resulting molecular signals, now with bandwidth 2.9-1.1 GHz, are filtered again with a $3 \mathrm{GHz}$ low-pass filter to eliminate the other sideband (11.0-12.8 GHz). The signals are then digitized on the oscilloscope, with its sampling rate reduced to $6.25 \mathrm{GS} / \mathrm{s}$ (Nyquist frequency $3.125 \mathrm{GHz}$ ). Because the record length has been reduced by a factor of 8 from the direct-digitized configuration described above, the nozzles can be run at $5 \mathrm{~Hz}$, allowing for 50 FIDs to be acquired per second, or 300,000 per hour. This reducedbandwidth configuration, as well as one from 9.1-13.6 GHz (in which the oscilloscope is run at $12.5 \mathrm{GS} / \mathrm{s}$ ), has been employed in our laboratory for multiple applications, including in the study of formic acid clusters as will be described below.

\section{c) CP-FTMW Stark effect measurements}

To measure molecular dipole moments, a Stark cage of the design of Emilsson et $a{ }^{7}{ }^{7}$ is used. The cage is a rectangular $53 \times 25 \times 33 \mathrm{~cm}$ plexiglass frame with sides consisting of wire grids, which are connected by a voltage divider consisting of eight 20 $\mathrm{M} \Omega$ resistors to provide good field homogeneity. Two high-voltage power supplies are attached to each side of the cage, and equal-magnitude positive and negative voltages are applied to the electrodes so that the field will be approximately zero in the center of the cage where the nozzle is applied. The electric field in the cage is calibrated using either the symmetric top molecule 3,3,3-trifluoropropyne (TFP, $\mu=2.319 \mathrm{D})^{8}$ or linear carbonyl sulfide, OCS $(\mu=0.719 \mathrm{D}){ }^{9}$ 
The advantage of TFP is that as a symmetric top, the levels exhibit first-order shifts, following the formula ${ }^{10}$

$$
E_{S}^{(1)}=-\frac{\mu \varepsilon K M_{J}}{J(J+1)}
$$

From this, the transition frequency for the $(J+1)_{K} \leftarrow J_{K}$ transition will be shifted by

$$
\Delta v=\frac{2 \mu \varepsilon K M_{J}}{h J(J+1)(J+2)}=1.00683 \mathrm{MHz} \mu(\mathrm{D}) \varepsilon\left(\frac{\mathrm{V}}{\mathrm{cm}}\right) \frac{K M_{J}}{J(J+1)(J+2)}
$$

Equation 3.2 assumes $\Delta M_{J}=0$ selection rules; these are determined by the relative orientation between the Stark electrode and the horn antennas. From these equations, it can be seen that first-order shifts only occur for energy levels where both $K$ and $M_{J}$ are nonzero. For a typical field strength of $80 \mathrm{~V} / \mathrm{cm}$, ideal for molecules with dipole moments on the order of 1 debye and 5-10 heavy atoms, TFP transition shifts are on the order of 5-50 MHz. With a typical frequency uncertainty of $\sim 10 \mathrm{kHz}$, this means that $\mu \cdot \varepsilon$ is determined to a precision of $\sim 10^{-3}$.

OCS, meanwhile, exhibits only second-order Stark shifts because it does not have a $K$ quantum number in its vibrational ground state. The expression for the Stark energy is

$$
E_{S}^{(2)}=\frac{\mu^{2} \varepsilon^{2}}{2 h B} \frac{J(J+1)-3 M_{J}^{2}}{J(J+1)(2 J-1)(2 J+3)}
$$

For the $J=1 \leftarrow 0$ transition of OCS at $12162.978 \mathrm{MHz}$, the only transition in the bandwidth of the 7.0-18.5 GHz spectrometer, the shift of the $M_{\mathrm{J}}=0 \leftarrow 0$ transition is

$$
\Delta v=5.74476 \times 10^{-6} \mathrm{MHz}\left(\varepsilon\left(\frac{\mathrm{V}}{\mathrm{cm}}\right)\right)^{2}
$$


At an electric field strength of $100 \mathrm{~V} / \mathrm{cm}$, therefore, the shift of this transition is only $57 \mathrm{kHz}$, which offers a poor determination of the electric field. However, for larger fields the shifts can be better determined, while the first-order TFP shifts can become too large $(>100 \mathrm{MHz})$ and field inhomogeneity can prevent these transitions from being observed. Therefore, when a large electric field is required, OCS should be used as the calibrant.

Typically, only a single voltage is needed to measure the dipole moments of all species in a sample. The fitting of the Stark-shifted spectra is performed using the QSTARK program of Kisiel. ${ }^{11}$ Ideally, the spectrum of the calibrant should be measured at the same time as the system under study; if this is not possible (because the signal of the calibrant interferes with that of other molecules in the sample), the two spectra should be measured consecutively, without powering off the high voltage supply in between. This approach eliminates any concern for the voltage accuracy of the high-voltage power supply; the spectrum of the calibrant reports the electric field strength, which is then used in the determination of molecular dipole moments. The spectrometer design is otherwise the same as for CP-FTMW spectroscopy as described above, except that only a single nozzle can be used; to date, we have observed that two nozzles do not experience the same electric field with the cages in the lab, and therefore their Stark-shifted FID signals do not co-add.

\section{d) Microwave-microwave double resonance spectroscopy}

As described in Chapter 2, microwave-microwave double-resonance (MW-MW DR) spectroscopy is a powerful technique to aid in spectral assignment by finding pairs 
of transitions that share a quantum state. Particularly in a dense spectrum where multiple species are present at the same signal level, these techniques are useful for extracting spectral assignments. Schematics of MW-MW DR implemented in a 7.0-18.5 CP-FTMW spectrometer and in a Balle-Flygare-type FTMW spectrometer are presented in Figure 3.3.

The CP-FTMW design is a straightforward extension of the schematic presented in Chapter 2. The only change with the spectrometer modifications presented above is that the multiple FIDs per valve pulse approach can be extended to perform DR on and DR off excitation/detection cycles (5 each) on every valve pulse. This is particularly valuable due to the fact that sample conditions can change as a function of time, and so this approach guarantees that the DR on and off pulses are performed with the same valve and sample conditions. As in the $2-8 \mathrm{GHz}$ demonstration, a single measurement provides all the transitions in the bandwidth of the CP-FTMW spectrometer that share a quantum state with the transition pumped by the double resonance pulse.

Double resonance spectroscopy in a cavity FTMW spectrometer using optical, ${ }^{12}$ infrared, ${ }^{13}$ and millimeter-wave ${ }^{14}$ double resonance sources has been previously described. Applying MW-MW DR spectroscopy in a cavity FTMW spectrometer can likewise be used to screen for resonances in a dense spectrum. A broadcast horn is oriented perpendicular to the cavity axis, through which the double resonance pulse is applied. As in the CP-FTMW-MW DR spectrometer, the double resonance pulse is applied immediately following the microwave excitation pulse. The typical approach is 

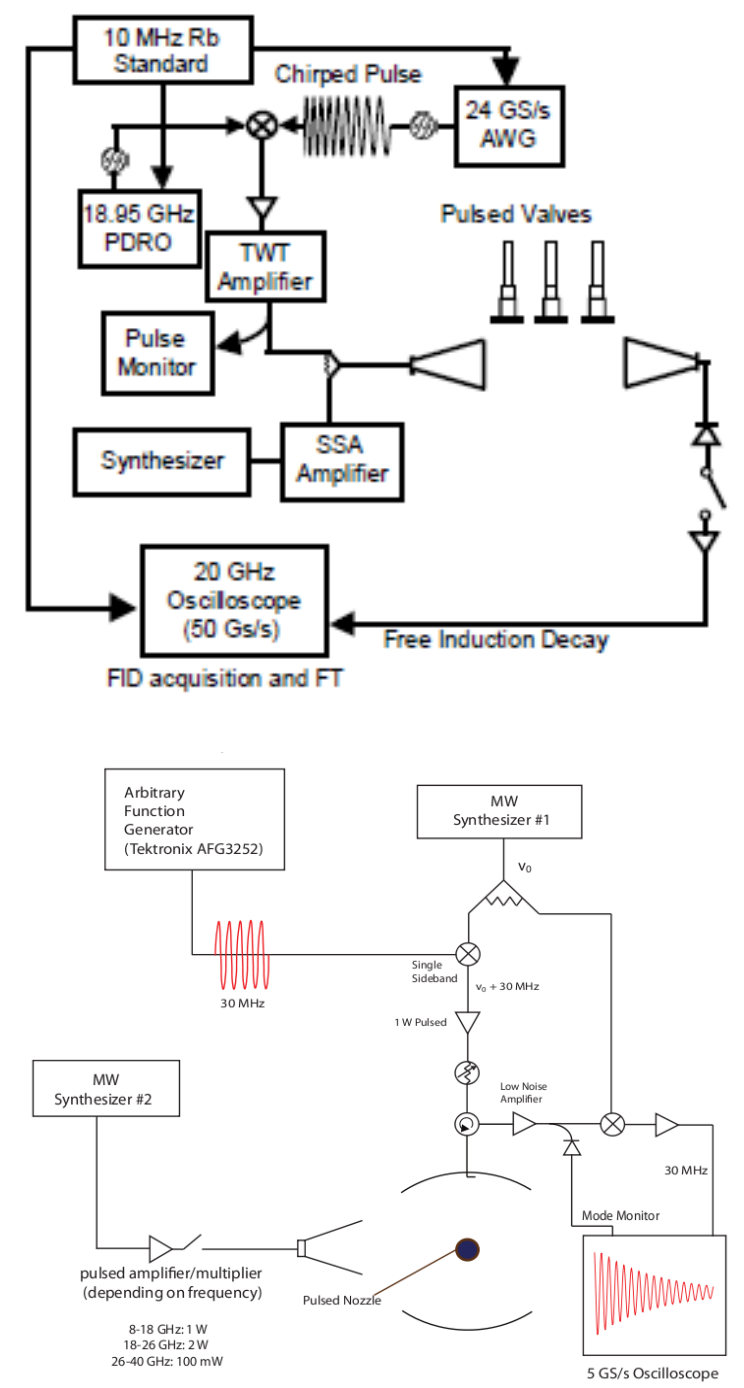

Figure 3.3. Schematics of microwave-microwave double resonance as implemented in a 7.0-18.5 GHz CP-FTMW spectrometer (top) and a Balle-Flygare-type cavity-FTMW spectrometer (bottom). 
to choose a strong, unassigned transition in a CP-FTMW spectrum to monitor. The double resonance pulse then cycles through a list of frequencies corresponding to a set of unassigned transitions against which to check for resonances. On each double resonance frequency, a set number of averages is performed (at least enough to reach a signal to noise level of at least $~ 5: 1$, typically) and the signal level recorded. Both the scanning of the double resonance frequency and the cavity monitor frequency can be automated so that this spectrometer can run for extended periods of time without user intervention.

\section{e) Automated fitting program}

In the past few years, in collaboration with Steven Shipman and Ian Finneran at the New College of Florida, we have developed a program that serves as a "structure screener" to perform automated fitting. The underlying idea is that for most molecules with simple rotational Hamiltonians (i.e., molecules that are relatively rigid and lack nuclear quadrupole hyperfine structure or internal rotation splitting), the spectrum can be described reasonably well at microwave frequencies by only the rotational constants, $A$, $B$, and $C$. Therefore, a reasonable spectral fit can be achieved for these molecules with only three transitions. The typical way to identify new molecules in CP-FTMW spectra is to use guess rotational constants (such as from an electronic structure calculation) to simulate a rotational spectrum in a graphical analysis program, then look for experimental transitions that are located near the predicted frequencies of strong calculated transitions. A few guess frequencies are then fit to the rotational constants. Based on the fit constants, the spectrum is predicted again. If the assigned transitions are 
correct, this will typically cause all of the other predicted transitions in the spectrum to fall near experimental transitions; from this point, the fitting process is usually very straightforward, usually involving only refinement with quartic distortion constants.

The idea of the program, therefore, is to automate this "guess and check" process. The user inputs guess rotational constant values and dipole moments (for simplicity, it is recommended to fit only transitions of a single dipole moment component in a search), and then selects three quantum number transitions to fit, and a set of other transitions (typically between 5-10) to check. Finally, the user selects a frequency window over which to search for candidate transitions, and minimum and maximum intensity thresholds. From this, a set of candidate frequencies is generated for each fit transition; all possible three-line combinations make up the list of iterations for the program to run. On each iteration, the program fits the three fit frequencies to $A, B$, and $C$, then simulates the spectrum based on the fit constants; if the predicted frequencies of each of the "check" transitions lie very near experimental transitions, the fit is judged to be successful. The program sorts the iterations by the root-mean-square deviation between the predicted and experimental transition frequencies. Written in Python, this program uses SPFIT/SPCAT for spectral simulation and fitting, and under typical conditions can run about 300,000 iterations per hour on a quad-core personal computer with a $2.67 \mathrm{GHz}$ processor.

There are a few aspects of the use of this program that warrant discussion. The first is that care must be taken in the choice of the transitions to fit; if the three fit transitions do not independently fit $A, B$, and $C$, the run will be unsuccessful. This can 
particularly be a problem for nearly symmetric species; for example, in a near-prolate $a$ type spectrum, $K_{a}=0$ transitions depend primarily on $B+C$, and so fitting three $K_{a}=0$ transitions would be unlikely to effectively screen for spectra. Therefore, it is typically useful to choose three transitions that are as uncorrelated as possible, such as transitions that vary considerably in $J$ and $K$, and particularly to avoid "paired" transitions (e.g., transitions with the same values of $J$ and one of the two $K$ s; the transition frequencies of these pairs are often highly correlated). A key parameter is evaluating whether three transitions are good choices is by calculating the determinant of the matrix of derivatives of the transition frequencies with respect to the rotational constants; that is,

$$
\left|\begin{array}{lll}
\frac{\partial v_{1}}{\partial A} & \frac{\partial v_{1}}{\partial B} & \frac{\partial v_{1}}{\partial C} \\
\frac{\partial v_{2}}{\partial A} & \frac{\partial v_{2}}{\partial B} & \frac{\partial v_{2}}{\partial C} \\
\frac{\partial v_{3}}{\partial A} & \frac{\partial v_{3}}{\partial B} & \frac{\partial v_{3}}{\partial C}
\end{array}\right|
$$

If this determinant of this matrix is zero, then the three chosen transitions cannot fit $A, B$, and $C$, because the three frequencies are not independent pieces of information. If the determinant is small (e.g., less than 1), then technically values of the rotational constants can be found, but the constants are likely to be highly correlated and therefore it is possible that the three-line fit constants may not be close to the actual constants (even if the three fit transition assignments are correct).

A second issue that is important to consider, particularly in the fitting of dense spectra, is the effect of blended lines. In CP-FTMW spectroscopy, because of the accuracy of the relative transition intensities, intensity screening is very important in 
determining candidate fit transitions. Therefore, when a transition of a species lies underneath a stronger line from another species, this transition might be omitted from the list of candidate frequencies on the basis of the anomalous intensity of the blend, which would likely result in the program missing the spectrum fit. Obviously, this becomes more of a problem as one attempts to fit weaker spectra, as it becomes more common for transitions to become blended. For extremely dense spectra such as the formic acid cluster spectrum presented below, this can become a serious concern. There are two possible ways to circumvent this problem. The first is to remove the maximum intensity threshold, so that a transition can be "fit" to a frequency that corresponds to an anomalously strong transition, even one that has been previously assigned to another species. The upside to this approach is that if a transition is obscured, a frequency that is near the actual molecular transition frequency of the species of interest will be included, from which the species could be found. However, if the blended transition is one of the three fit transitions, this will increase the deviation between the fit rotational constants from the triplet and the actual constants, because the frequency of the blended transition is different from that of the isolated species. The second way is to do multiple iterations of the program with different fit transitions, to reduce the possibility of a fit being missed due to blended transitions. This can be more time intensive, but is more thorough in the evaluation of whether a proposed structure is present in a spectrum. The recommendation of the author is that strong transitions be included as "check" transitions, but not as fit transitions; the inclusion of these transitions as potential fits increases the number of iterations of the program that must be performed, with minimal 
gain due to the fact that the errors in the rotational constants introduced by fitting a blend typically limit its usefulness. Additionally, the fitting of at least two sets of transitions should be performed if this is possible; particularly in the fitting of weak transitions, occasional intensity anomalies can occur, and the fitting of multiple sets of transitions reduces the effect of these fluctuations, and increases the chances of a spectrum being located by this program if it is present.

The program also is able to take other Hamiltonian parameters, particularly centrifugal distortion constants, as input. These constants are not fit in the current version of the program, but as will be demonstrated below, this is particularly important for the fitting of isotopologues. These species can be difficult to pick out of dense spectra due to their weak intensities. Additionally, when the structure of the species is not accurately known, the isotopologue transition frequencies cannot be accurately estimated. Because the distortion constants of isotopologues are typically very close to those of the normal species, including the normal species distortion constants in the isotopologue search makes it possible for the automated fitting program to fit these isotopologues to experimental uncertainty $(\sim 10 \mathrm{kHz}$ deviation or less) with three transitions.

For molecules with more complex Hamiltonians, the automated fitting program can be adjusted to fit additional parameters, but since the number of fit lines must match the number of fit parameters, the computational time required will increase exponentially. It should be noted, however, that most molecules that have been studied in our lab could be found using this program. For molecules with internal methyl rotation, for example, 
the $A$ symmetry species can be fit to an effective rigid rotor Hamiltonian, so at least the initial spectrum could be found by this program. Additionally, it should be noted that many other effects that complicate rotational spectra, such as nuclear quadrupole splitting and tunneling, are minimal in larger molecules, and so for the majority of systems one wishes to study by rotational spectroscopy this program can be very useful.

\section{Structure Determination of a Large, Floppy Molecule: Dispersive Interactions in Strawberry Aldehyde ${ }^{15}$ \\ a) Introduction}

Large molecules are often marked by rich conformational landscapes. Because of the close relationship between the structure of a molecule and its biological function, the characterization of the low-energy conformations of large molecules, and the energy barriers that separate them, is important to understanding a molecule's role in biological processes. These conformational landscapes can be probed using electronic structure theory. However, for larger systems these studies are challenging, because the number of possible conformers increases exponentially with molecular size. ${ }^{16}$ Additionally, for larger molecules, dispersive, long-range interactions begin to wield large influences over the conformational landscape, and these interactions are often difficult to characterize accurately by ab initio calculations.

For this reason, the CP-FTMW approach is a good one for the characterization of large, conformationally flexible molecules. Because of its unbiased, broadband nature, all species with dipole moments in the sample will be observed, eliminating the need for 
accurately calculated rotational constants to guide searches. Through the assignment of isotopically labeled species in natural abundance, the structure of the assigned conformers can be determined purely experimentally. With direct structural information, the conformational structure can be determined in the absence of electronic structure information. This also allows for different theoretical methods to be directly tested experimentally, which is a main focus of the project presented here.

In this section, the conformational potential energy surface of ethyl 3-methyl-3phenylglycidate $\left(\mathrm{C}_{12} \mathrm{H}_{14} \mathrm{O}_{3}\right.$, commonly referred to as strawberry aldehyde $)$ is explored. This molecule contains 15 heavy atoms, and possesses two chiral centers, and so conformational isomers of this molecule are either homochiral (cis) or heterochiral (trans). It should be noted that enantiomers are not resolvable by microwave spectroscopy, and so, for example, the $(R, R)$ and $(S, S)$ forms are not distinguishable, but because diastereomers have different three-dimensional mass distributions, they can be resolved. Strawberry aldehyde is particularly interesting in that the two conformational families have qualitatively different potential energy surfaces due to the fact that in the homochiral (cis) diastereomer, the terminal alkyl chain is able to interact with the phenyl ring via a through-space dispersion interaction, but in the heterochiral (trans) diastereomer, the molecule is insufficiently flexible for this interaction to substantially affect the molecular energy.

The lowest-energy conformers of cis and trans are presented in Figure 3.4. Each conformational family has 12 possible isomers, for a total of 24 possible conformers. 

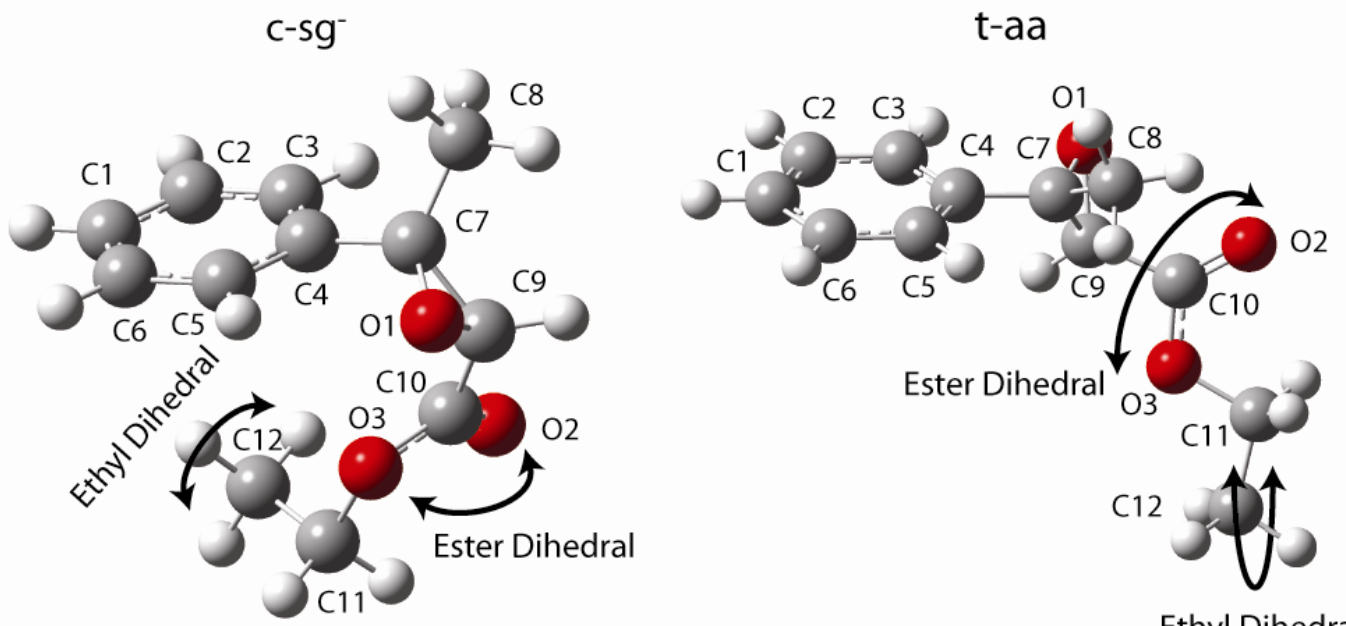

Ethyl Dihedral

Figure 3.4. The two dominant conformers of strawberry aldehyde, $\mathrm{c}^{-\mathrm{sg}^{-}}{ }^{-}$and $\mathrm{t}$-aa. The indicated ester and ethyl dihedral angles are the two primary angles used to differentiate the 12 lowest energy conformers. In both structures, the ester dihedral involves O1, C9, $\mathrm{C} 10$, and $\mathrm{O} 3$, and the ethyl dihedral involves $\mathrm{C} 10, \mathrm{O} 3, \mathrm{C} 11$, and $\mathrm{C} 12$. 
The three coordinates of conformational flexibility are rotation around the $\mathrm{C} 9-\mathrm{C} 10$ bond, the $\mathrm{C} 10-\mathrm{O} 3$ bond, and the $\mathrm{O} 3-\mathrm{C} 11$ bond. Conformations in which the $\mathrm{C} 10-\mathrm{O} 3$ dihedral is syn are far more energetically favorable than anti conformers in this coordinate; this is a common property of esters. ${ }^{17-19}$ Therefore, the conformational surface of strawberry aldehyde is dominated by the 6 isomers of each family that are syn in the C10-O3 dihedral. These conformers are labeled $\mathrm{x}-\mathrm{yz}$, where $\mathrm{x}$ designates the stereochemistry about the epoxide (c for cis, $\mathrm{t}$ for trans), y designates the orientation about the C9-C10 bond ( $\mathrm{s}$ for syn, a for anti), which is referred to as the ester dihedral; and $\mathrm{z}$ indicates the orientation of the terminal ethyl group, rotation about the $\mathrm{O} 3-\mathrm{C} 11$ bond (a for anti, $\mathrm{g}^{+}$for gauche with a dihedral angle of $\sim 80^{\circ}, \mathrm{g}^{-}$for gauche with a dihedral angle of $\sim-80^{\circ}$ ), which is referred to as the ethyl dihedral. Strawberry aldehyde was chosen for this benchmark measurement of CP-FTMW spectroscopy because it is a large molecule with a rich conformational landscape that is inexpensive, readily available, and easy to handle. Additionally, structural studies of strawberry aldehyde may be of interest to understanding olfactory response to fragrances and has been the subject of a previous study relating olfactory potency to overall stereochemistry and structural modifications. ${ }^{20}$

\section{b) Experimental}

A sample of strawberry aldehyde was obtained from Sigma-Aldrich $(\geq 98 \%$ purity, equal mixture of isomers) and used without further purification. The rotational spectrum of strawberry aldehyde was measured from 7.0-18.5 GHz with a CP-FTMW spectrometer as described above. Two pulsed nozzles fitted with reservoirs were used, 
with 10 FID signals collected per valve pulse. The conditions for the supersonic expansion were a reservoir temperature of $120^{\circ} \mathrm{C}$ and a mixed $\mathrm{He} / \mathrm{Ne}$ carrier gas (30:70 He:Ne, "first run neon") with a backing pressure of 1 atm. The spectrum presented here is the Fourier transform of 929,000 FIDs, each with a $20 \mu$ s duration; after the application of a Kaiser-Bessel window function, the transitions have a full-width at half maximum (FWHM) of $120 \mathrm{kHz}$. It was acquired in 42.6 hours of continuous averaging and consumed approximately $400 \mathrm{mg}$ of sample.

The most intense transition in the CP-FTMW spectrum of strawberry aldehyde has a signal-to-noise ratio of approximately 1,000:1. The spectrum is shown in Figure 3.5. A total of 8908 transitions with a signal-to-noise ratio greater than 3:1 are observed, of which 1925 have been assigned to the rotational spectra of 5 normal species conformers, $24{ }^{13} \mathrm{C}$ isotopomers in natural abundance (each of the 12 singly-substituted isotopomers of the 2 dominant conformers), and thermal decomposition products. Rotational Hamiltonian parameters for the five assigned conformers are reported in Table

\section{1.}

\section{c) Structural characterization of the $c-s g^{-}$and $t$-aa conformers}

For the two conformers for which the singly substituted ${ }^{13} \mathrm{C}$ isotopologues were assigned (c-sg- and t-aa), Kraitchman's equations were applied to determine the coordinates of each carbon atom with respect to the molecular center-of-mass in the principal axis system. In a few cases, the substituted carbons were located near a 

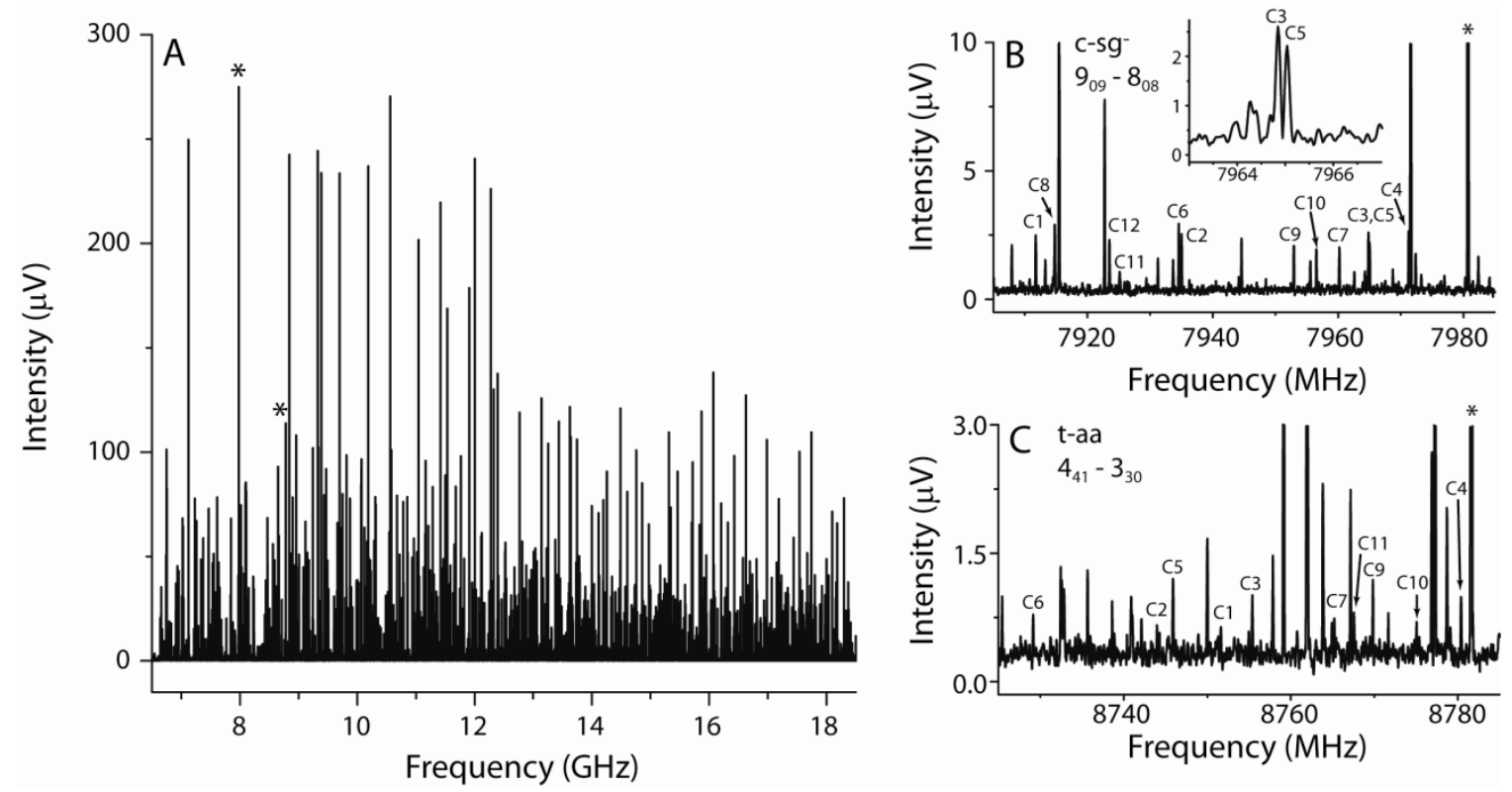

Figure 3.5. Panel A shows the rotational spectrum of strawberry aldehyde from 6.5-18.5 GHz. The noise level of this spectrum is $\sim 0.3 \mu \mathrm{V}$. The two marked peaks are the c-sg$9_{09}-8_{08}$ and the t-aa $4_{41}-3_{30}$ transitions at 7980.75 and $8781.62 \mathrm{MHz}$, respectively. Panels $\mathrm{B}$ and $\mathrm{C}$ show the satellite lines of the ${ }^{13} \mathrm{C}$ isotopomers of these transitions (asterisks indicate the normal species transitions that are off scale). Panel $\mathrm{B}$ also includes an inset of the ${ }^{13} \mathrm{C}-3$ and ${ }^{13} \mathrm{C}-5 \mathrm{c}-\mathrm{sg}^{-}$transitions showing that they are resolved in the spectrum. In panel $\mathrm{C}$, the $4_{41}-3_{30}$ transitions from the ${ }^{13} \mathrm{C}-8$ and ${ }^{13} \mathrm{C}-12$ isotopomers of the $\mathrm{t}$-aa conformer are not shown in the interest of clarity as they are both located at $\sim 60 \mathrm{MHz}$ lower frequency than those of the other isotopomers. 
Table 3.1. Rotational $S$-reduction Hamiltonian parameters for the five assigned conformers of strawberry aldehyde.

\begin{tabular}{cccccc}
\hline & $\mathrm{c}-\mathrm{sg}^{-}$ & ${\mathrm{c}-\mathrm{ag}^{+}}^{+}$ & $\mathrm{t}-\mathrm{aa}$ & $\mathrm{t}-\mathrm{ag}^{-}$ & $\mathrm{t}-\mathrm{ag}^{+}$ \\
\hline$A(\mathrm{MHz})$ & $728.09520(17)$ & $723.14170(25)$ & $1214.7279(4)$ & $1460.5230(11)$ & $1330.9492(7)$ \\
$B(\mathrm{MHz})$ & $628.69170(12)$ & $581.41140(18)$ & $287.76660(8)$ & $275.045860(20)$ & $293.48150(12)$ \\
$C(\mathrm{MHz})$ & $429.84840(20)$ & $421.96270(28)$ & $269.46240(7)$ & $269.61760(19)$ & $281.86590(13)$ \\
$D_{J}(\mathrm{kHz})$ & $0.0808(7)$ & $0.3430(9)$ & $0.01740(7)$ & $0.01080(28)$ & $0.01720(13)$ \\
$D_{J K}(\mathrm{kHz})$ & $-0.1070(18)$ & $-0.863(4)$ & $-0.0770(10)$ & $-0.050(3)$ & $-0.0820(17)$ \\
$D_{K}(\mathrm{kHz})$ & $0.0900(24)$ & $0.656(5)$ & $0.820(10)$ & $1.350(23)$ & $0.950(20)$ \\
$d_{1}(\mathrm{~Hz})$ & $-25.6(7)$ & $-119.3(8)$ & $-3.410(29)$ & $-0.91(5)$ & $-3.14(4)$ \\
$d_{2}(\mathrm{~Hz})$ & $2.3(4)$ & $8.6(4)$ & $0.800(11)$ & $0.700(19)$ & $-0.750(18)$ \\
$\#$ lines & 280 & 229 & 256 & 138 & 193 \\
Max J & 16 & 16 & 30 & 28 & 28 \\
Max K & 12 & 10 & 6 & 6 & 5 \\
RMS $(\mathrm{kHz})$ & 12.9 & 13.7 & 11.2 & 14.5 & 15.6 \\
\hline
\end{tabular}


principal axis of the molecule; for these substitutions, the coordinate was constrained to zero to avoid unphysical (imaginary) atomic coordinates. The three singly-substitued ${ }^{18} \mathrm{O}$ isotopomers were not assignable at the sensitivity of this spectrum. As this analysis only determines the magnitudes of the atom positions, the signs of the coordinates must be inferred using assumptions about atomic connectivity; this is an important issue that will be explored further for the formic acid clusters in Section IV.

Least-squares fits of the moments of inertia for the 13 isotopomers assigned for each conformer (39 experimentally determined parameters) to internal coordinates of the molecule were also performed. Successful fits of large molecular structures using this approach requires care, because the isotopic information is incomplete. Since no isotopic information on the oxygen or hydrogen atoms in the molecule is known, most internal parameters involving these atoms must be held fixed to their $a b$ initio values, particularly for the positions of the hydrogen atoms. Additionally, the values of some pairs of internal coordinates are highly correlated and therefore cannot be independently fit. This input from theory can have the impact of diluting the ability to directly compare the experimental and $a b$ initio structures. Nevertheless, satisfactory structural fits were found with a total of 21 parameters ( 8 bond lengths, 6 bond angles, and 7 dihedral angles) for the c-sg- conformer, and 13 parameters ( 3 bond lengths, 5 bond angles, and 5 dihedral angles) for the t-aa conformer. The two methods give very similar structures, with a rootmean-square deviation of approximately $0.1 \AA$ between the two structures in the coordinates of the carbon atoms in the principal axis system. The two experimental 
structures derived from this isotopic data is presented in Figure 3.6, compared to ab initio calculated structures using density functional theory (using both the B3LYP and M05-2X functionals) and MP2 methods.

In the B3LYP calculated structure of the $\mathrm{c}^{-\mathrm{sg}^{-}}$conformer, the ethyl chain at the end of the molecule (C11-C12) is approximately $0.5 \AA$ farther from the phenyl ring than in the experimental structure. The M05-2X and MP2 calculated structures, on the other hand, agree with the experimental structure (r.m.s. atom position deviations between theory and experiment are $0.08 \AA$ and $0.15 \AA$ for M05-2X and MP2, respectively; the r.m.s. deviation for the B3LYP structure is $0.38 \AA$ ). Meanwhile, for the t-aa conformer, where a long-range interaction between the phenyl ring and the terminal alkyl chain is not present, all three calculated structures agree with the experimentally derived structure (r.m.s. position deviations between theory and experiment are $0.15 \AA, 0.06 \AA$, and $0.11 \AA$ for B3LYP, M05-2X, and MP2, respectively).

The primary difference between the B3LYP and M05-2X functionals is that the latter was specifically designed to account for long-range dispersive interactions. ${ }^{21-23}$ Due to the relatively close approach of the terminal alkyl chain to the phenyl ring in the cis conformers, the interaction between these groups plays an important role in the conformer energies and interconformer barrier heights. In the trans conformers, on the other hand, these two groups are well-separated and so interactions between them do not significantly affect the potential energy surface. In support of these observations, it 

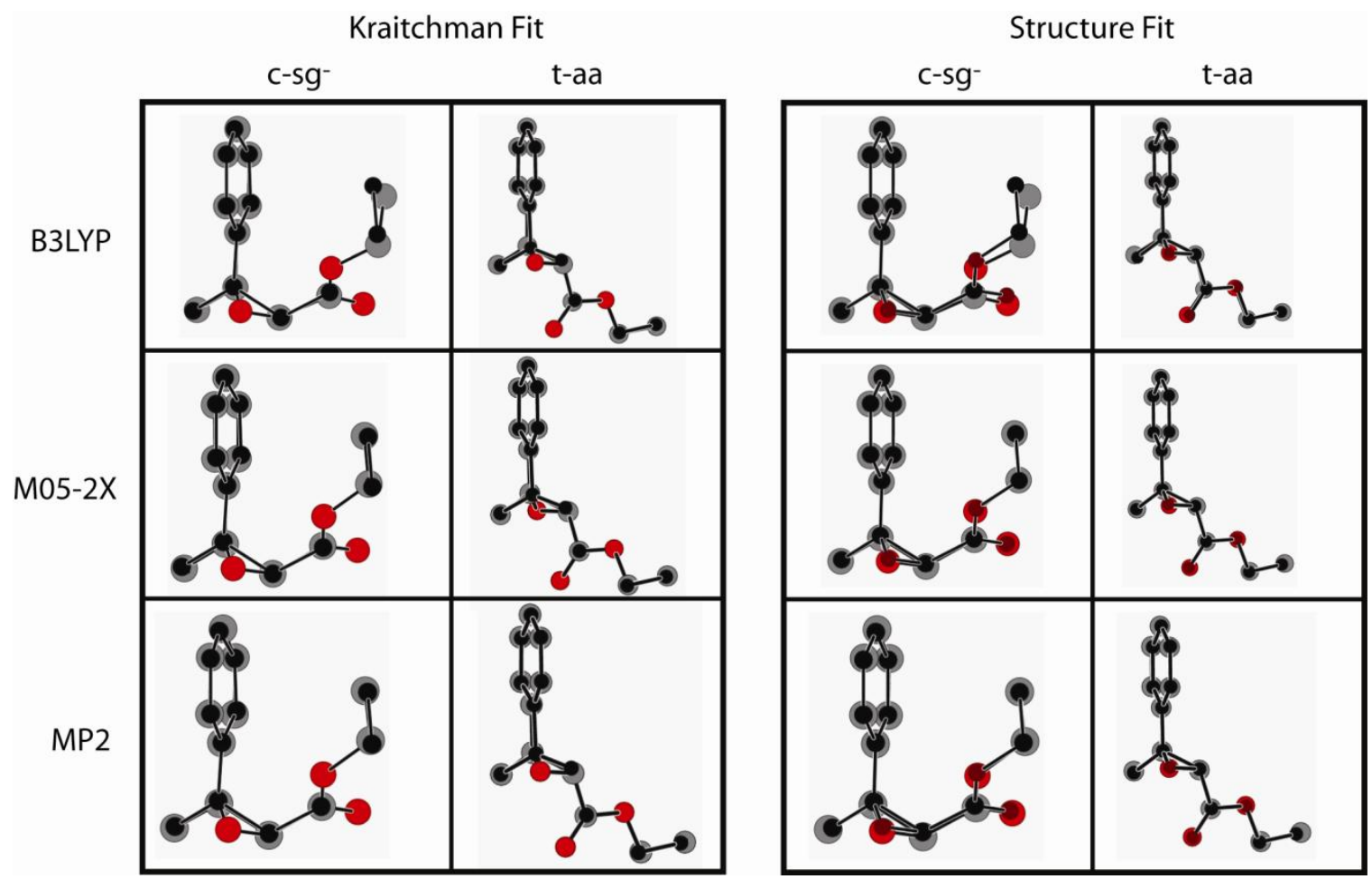

Figure 3.6. Renderings of the experimentally derived structures (black circles for carbon, maroon for oxygen) of the $\mathrm{c}^{-\mathrm{sg}^{-}}$and $\mathrm{t}$-aa conformers of strawberry aldehyde, using both the Kraitchman approach (left) and a least-squares structure fit (right). These structures are compared to calculated atom positions (carbon in gray, oxygen in red) at the B3LYP/6-311++G(d,p) (top), M05-2X/6-311++G(d,p) (middle), and MP2/6-31+G(d) (bottom) levels of theory. The calculated atom positions have diameter $0.7 \AA$, while the substitution coordinates have diameter $0.45 \AA$. The structures are oriented so the phenyl rings are overlaid. 
should be noted that the B3LYP functional has previously been shown to poorly describe systems with these kinds of interactions, ${ }^{24-26}$ and recent reports suggest that M05-2X performs markedly better than B3LYP on systems where dispersion is important. ${ }^{27-29}$

\section{d) Potential energy surface of strawberry aldehyde}

The sensitivity of the spectrum was not high enough to observe the ${ }^{13} \mathrm{C}$ isotopomers of the other three assigned conformers. Structural assignments of these conformers were therefore derived from a correlation of observed rotational constants and relative $a, b$, and $c$-type transition intensities to $a b$ initio values of these parameters for local minima. Table 3.2 shows these comparisons. Qualitative agreement of relative transition intensities, coupled with close agreement between calculated and experimental rotational constants (with the notable exception of B3LYP values in the cis conformations), confirm the assignments of the remaining conformers.

Two-dimensional relaxed potential energy surfaces, presented in Figure 3.7, offer insight into the effect of the dispersive interaction on the structure and interconformer dynamics of strawberry aldehyde. In these surfaces, calculated using both the B3LYP and M05-2X functionals, the ester and ethyl dihedral angles were fixed to points on a grid $\left(15^{\circ}\right.$ spacing) while the rest of the structure was allowed to relax to its lowest-energy configuration. To reduce computation time, these calculations were performed with a 6-31G(d) basis set and so should be viewed as qualitative. Based on their calculated relative energies, all 12 conformers are expected to have substantial populations in 
Table 3.2. Calculated and experimental rotational constants for the assigned conformers of strawberry aldehyde.

\begin{tabular}{|c|c|c|c|c|c|c|c|}
\hline \multirow[t]{2}{*}{ Conformer } & \multirow[t]{2}{*}{ Method $^{a}$} & \multirow[t]{2}{*}{$\mathrm{A}(\mathrm{MHz})$} & \multirow[t]{2}{*}{$\mathrm{B}(\mathrm{MHz})$} & \multirow[t]{2}{*}{$\mathrm{C}(\mathrm{MHz})$} & \multicolumn{3}{|c|}{ Normalized Transition Intensity ${ }^{b}$} \\
\hline & & & & & $a$-type & $b$-type & $c$-type \\
\hline \multirow{4}{*}{$c-\mathrm{sg}^{-}$} & Experiment & $728.09520(17)$ & $628.69170(12)$ & $429.84840(20)$ & 0.85 & 0.15 & 0 \\
\hline & B3LYP & 726.6 & 576.7 & 403.6 & 0.65 & 0.35 & 0 \\
\hline & M05-2X & 735.8 & 637.2 & 436.0 & 0.91 & 0.09 & 0 \\
\hline & MP2 & 727.0 & 634.7 & 434.4 & 0.93 & 0.06 & 0.01 \\
\hline \multirow{4}{*}{$\mathrm{c}-\mathrm{ag}^{+}$} & Experiment & $723.14170(25)$ & $581.41140(18)$ & $421.96270(28)$ & 0 & 0.08 & 0.92 \\
\hline & B3LYP & 772.8 & 476.8 & 374.8 & 0.05 & 0.08 & 0.87 \\
\hline & M05-2X & 720.6 & 612.7 & 436.5 & 0 & 0.13 & 0.87 \\
\hline & MP2 & 712.2 & 604.5 & 431.2 & 0 & 0.12 & 0.88 \\
\hline \multirow{4}{*}{ t-aa } & Experiment & $1214.7279(4)$ & $287.76660(8)$ & $269.46240(7)$ & 0 & 0.86 & 0.14 \\
\hline & B3LYP & 1214.2 & 285.3 & 264.2 & 0 & 0.92 & 0.08 \\
\hline & M05-2X & 1225.4 & 288.8 & 270.9 & 0 & 0.88 & 0.12 \\
\hline & MP2 & 1215.5 & 291.8 & 265.5 & 0 & 0.96 & 0.04 \\
\hline \multirow{4}{*}{ t-ag } & Experiment & $1460.5230(11)$ & $275.04860(20)$ & $269.61760(19)$ & 0 & 0.71 & 0.29 \\
\hline & B3LYP & 1442.8 & 272.6 & 265.1 & 0 & 0.84 & 0.16 \\
\hline & M05-2X & 1484.7 & 276.3 & 270.9 & 0 & 0.45 & 0.55 \\
\hline & MP2 & 1459.8 & 277.8 & 266.7 & 0.01 & 0.94 & 0.05 \\
\hline \multirow{4}{*}{${\mathrm{t}-\mathrm{ag}^{+}}^{+}$} & Experiment & $1330.9492(7)$ & $293.48150(12)$ & $281.86590(13)$ & 0 & 0.94 & 0.06 \\
\hline & B3LYP & 1317.5 & 289.4 & 274.6 & 0 & 0.99 & 0.01 \\
\hline & M05-2X & 1344.0 & 293.7 & 284.2 & 0 & 0.93 & 0.07 \\
\hline & MP2 & 1337.1 & 298.2 & 277.7 & 0.01 & 0.98 & 0.01 \\
\hline
\end{tabular}

\footnotetext{
${ }^{a}$ B3LYP and M05-2X calculations were performed with a 6-311++G(d,p) basis set, while the MP2 calculations were performed with a $6-31+\mathrm{G}(\mathrm{d})$ basis set.

${ }^{b}$ Calculated transition intensities are determined assuming quadratic scaling of intensity with dipole moment.
} 

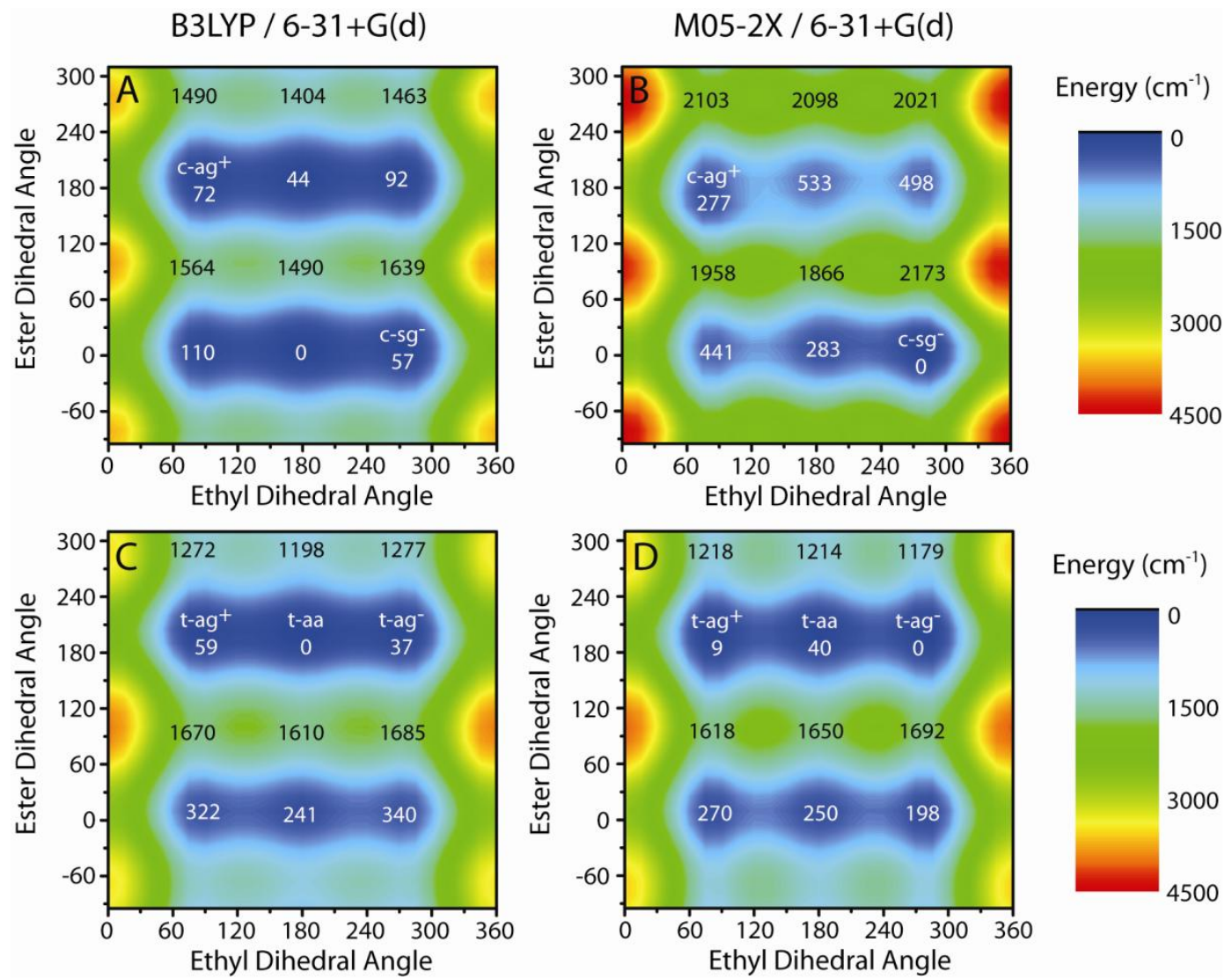

Figure 3.7. Relaxed potential energy surfaces in two dihedral angles for the cis (panels A and B) and trans (panels C and D) families of strawberry aldehyde conformers, calculated at the B3LYP/6-31G+(d) (panels A and C) and the M05-2X/6-31+G(d) (panels $\mathrm{B}$ and D) levels of theory. Potential wells corresponding to observed conformers are labeled with their conformer designations. The ester and ethyl dihedrals are as shown in Figure 1. Numbers on the plot indicate the energies $\left(\right.$ in $\mathrm{cm}^{-1}$ ) of different conformers and of interconformer transition states along the ester dihedral coordinate, relative to the most stable conformer on each surface. 
thermal equilibrium at $120{ }^{\circ} \mathrm{C}$, the sample temperature before the supersonic expansion, but cooling in the pulsed jet causes some of the conformers to relax to lower-energy minima, depending on the barriers to relaxation. ${ }^{30}$ Using the relative intensities observed in the CP-FTMW spectrum, populations for each of the experimentally observed isomers can be determined. The calculated relative energies of the 12 conformers and their experimental populations in the supersonic expansion are presented in Tables 3.3 and 3.4.

The two-dimensional potential energy surfaces reveal clear differences in the relative energies and barrier heights for conformers depending on the calculation method. On the B3LYP/6-31+G(d) surface, the two observed cis conformers have intermediate relative energies, and so other conformers should have been present at higher abundances than the two observed were this surface correct. The M05-2X surface calculates that the two observed cis conformers, in which the methyl group is oriented facing the phenyl ring, are stabilized relative to the others with the same ester dihedral angle. It has a higher barrier to changing the ester dihedral angle than the B3LYP surface (by roughly $500 \mathrm{~cm}^{-1}$ ), which makes it more likely to trap molecules in a particular ester configuration, with conformational cooling only occurring in the relatively low barrier ethyl dihedral coordinate. Our observation is in better agreement with the M05-2X surface than with the B3LYP surface. This explains why, in Table 3.4, the c-sa conformer is not observed experimentally while the $\mathrm{c}-\mathrm{ag}^{+}$conformer is, despite the c-sa conformer being more stable; c-sa can cool with a low barrier in the expansion to the minimum, c-sg; the ${\mathrm{c}-\mathrm{ag}^{+}}^{-}$conformer, meanwhile, cannot cool easily to lower-energy conformers. 
Table 3.3. Relative energies, ${ }^{a}$ total dipole moments, dihedral angles, ${ }^{b}$ and normalized populations $^{c}$ of the six lowest trans conformers of strawberry aldehyde at the M05-2X/6$311++\mathrm{G}(\mathrm{d}, \mathrm{p})$ level of theory.

\begin{tabular}{ccccccc}
\hline Conformer & Rel. E $\left(\mathrm{cm}^{-1}\right)$ & $\begin{array}{c}\text { Calculated } \\
\text { Population } \\
\left(120^{\circ} \mathrm{C}\right)\end{array}$ & $\mu_{\mathrm{tot}}(\mathrm{D})$ & $\begin{array}{c}\text { Ester Dihedral } \\
\text { (degrees) }\end{array}$ & $\begin{array}{c}\text { Ethyl Dihedral } \\
\text { (degrees) }\end{array}$ & $\begin{array}{c}\text { Experimental } \\
\text { Population }\end{array}$ \\
\hline $\mathrm{t}$-aa & 0 & 0.24 & 3.4 & 195 & 180 & $57 \%$ \\
$\mathrm{t}-\mathrm{ag}$ & 16 & 0.23 & 3.1 & 195 & 279 & $18 \%$ \\
$\mathrm{t}-\mathrm{ag}^{+}$ & 28 & 0.22 & 3.4 & 194 & 81 & $24 \%$ \\
$\mathrm{t}-\mathrm{sg}$ & 209 & 0.11 & 1.8 & 5 & 279 & - \\
$\mathrm{t}-\mathrm{sa}$ & 226 & 0.11 & 1.7 & 6 & 181 & - \\
$\mathrm{t}-\mathrm{sg}^{+}$ & 257 & 0.09 & 1.4 & 6 & 81 & - \\
\hline
\end{tabular}

${ }^{a}$ Energies have been corrected for zero-point vibrational energy.

${ }^{b}$ Dihedral angles are indicated in Figure 1.

${ }^{c}$ Experimental populations are determined by dividing the total intensity (summing $a-, b-$, and $c$-types) by the square of the total dipole moment at an M05-2X level of theory. 
Table 3.4. Relative energies, ${ }^{a}$ total dipole moments, dihedral angles, ${ }^{b}$ and normalized populations $^{c}$ of the six lowest cis conformers of strawberry aldehyde at the M05-2X/6$311++\mathrm{G}(\mathrm{d}, \mathrm{p})$ level of theory.

\begin{tabular}{ccccccc}
\hline Conformer & Rel. E $\left(\mathrm{cm}^{-1}\right)$ & $\begin{array}{c}\text { Calculated } \\
\text { Population } \\
\left(120^{\circ} \mathrm{C}\right)\end{array}$ & $\mu_{\text {tot }}(\mathrm{D})$ & $\begin{array}{c}\text { Ester Dihedral } \\
(\text { degrees })\end{array}$ & $\begin{array}{c}\text { Ethyl Dihedral } \\
\text { (degrees) }\end{array}$ & $\begin{array}{c}\text { Experimental } \\
\text { Population }\end{array}$ \\
\hline $\mathrm{c}-\mathrm{sg} \mathrm{g}^{-}$ & 0 & 0.35 & 2.0 & 2 & 280 & $88 \%$ \\
$\mathrm{c}-\mathrm{sa}$ & 79 & 0.26 & 2.1 & 8 & 189 & - \\
$\mathrm{c}^{-\mathrm{ag}^{+}}$ & 273 & 0.13 & 3.5 & 165 & 79 & $12 \%$ \\
${\mathrm{c}-\mathrm{sg}^{+}}^{+}$ & 324 & 0.11 & 1.7 & 5 & 83 & - \\
$\mathrm{c}^{-a a}$ & 391 & 0.08 & 3.4 & 181 & 180 & - \\
$\mathrm{c}^{-a g}$ & 475 & 0.06 & 3.2 & 182 & 278 & - \\
\hline
\end{tabular}

${ }^{a}$ Energies have been corrected for zero-point vibrational energy.

${ }^{b}$ Dihedral angles are indicated in Figure 1.

${ }^{c}$ Experimental populations are determined by dividing the total intensity (summing $a-, b$ , and $c$-types) by the square of the total dipole moment at an M05-2X level of theory. 
For the trans conformers, the M05-2X and B3LYP surfaces are qualitatively similar. The barrier to cooling along the ester dihedral angle is lower than for the cis M05-2X surface, and only the anti configuration in this angle is observed experimentally. This is surprising in light of the study of Ruoff et al., ${ }^{30}$ since $31 \%$ of the population of trans is calculated to be in the syn ester configuration before the supersonic expansion, and the barrier to cooling along the ester dihedral is $\sim 1500 \mathrm{~cm}^{-1}$. The three conformers with an anti configuration are all observed despite low barriers to cooling between them. This is likely due to the fact that their relative energies are extremely close, and so even at a relatively low conformational temperature they would be expected to be populated.

\section{e) Conclusion}

An analysis of the rotational spectrum of strawberry aldehyde using highsensitivity CP-FTMW spectroscopy resulted in the assignment of 5 conformers of strawberry aldehyde, 2 of cis and 3 of trans. Strawberry aldehyde is sufficiently flexible that intramolecular interactions between functional groups of the molecule are present. In this situation, the B3LYP density functional adequately described the conformers of trans-strawberry aldehyde, but did not properly handle the cis conformers, whose energies and interconversion barriers are strongly influenced by a long-range interaction between an alkyl group and a phenyl ring. This is demonstrated by an experimental heavy-atom structure using assignments of each singly-substituted ${ }^{13} \mathrm{C}$ isotopomer of the most populated conformers to derive atom positions. The M05-2X density functional, as

well as MP2 methods, appropriately treated the intramolecular interaction in the 
conformers of cis, and excellent agreement between calculated and experimental structures was observed.

This molecule demonstrates some of the difficulties that will become more commonplace as high-resolution spectroscopies are used to study ever-larger systems. As molecules increase in size, they have a larger number of populated conformers, and some fraction of these conformers will certainly be stabilized by intramolecular interactions that are poorly treated by the theoretical methods currently in use by experimentalists. The CP-FTMW technique, which provides sensitivity sufficient to obtain heavy atom molecular structures from samples in natural abundance with low sample consumption, will be essential in allowing experimental results to inform the development of new theoretical methods.

\section{Structure Determination of Clusters of Formic Acid}

\section{a) Introduction}

In this section, the application of high-sensitivity CP-FTMW spectroscopy to the study of weakly bound clusters is described. The interrogation of weakly bound complexes by high-resolution spectroscopy coupled to supersonic expansion techniques is a field with a long history. ${ }^{31,32}$ The unbiased nature of CP-FTMW spectroscopy, and the direct structural information that can be obtained, makes it a powerful way to examine the structure and dynamics of these complexes. These studies are important for understanding the long-range interactions that often determine the structures of weakly bound complexes, which, as described in the previous section, often pose challenges for 
electronic structure theory. These complexes also present a bridge between structure determination of completely isolated, gas-phase molecules, and liquid phase structure and dynamics, which are strongly affected by solute-solvent interactions.

Formic acid was chosen for this study. The gas-phase vapor of formic acid is dominated by a non-polar, eight-membered cyclic dimer with two O-H...O hydrogen bonds, which does not have a pure rotational spectrum. This complex exhibits rapid tunneling of the two hydrogen-bound protons between the two monomer units. The structure and dynamics of this complex have been the subject of numerous experimental and theoretical studies. ${ }^{33}$ There is a higher-energy form of the formic acid dimer that contains a seven-membered ring (with the formic acid units connected by one O-H... and one $\mathrm{C}-\mathrm{H} . . . \mathrm{O}$ interaction); experimental evidence for the presence of this species by gas-phase Raman spectroscopy ${ }^{34}$ and by infrared spectroscopy $^{35}$ within helium nanodroplets has been presented. The interactions in this polar dimer have been suggested to play an important role in the dimerization of formic acid ${ }^{36}$ and in its structure in the liquid and solid phases. ${ }^{37,38}$

The structures of complexes between formic acid and water have been the subject of a previous investigation by microwave spectroscopy. ${ }^{39}$ In this study, the rotational spectra of the $\mathrm{HCOOH}-\mathrm{H}_{2} \mathrm{O}$, $(\mathrm{HCOOH})_{2}-\mathrm{H}_{2} \mathrm{O}$, and $\mathrm{HCOOH}-\left(\mathrm{H}_{2} \mathrm{O}\right)_{2}$ complexes were assigned, and their structures were determined by comparisons between calculated and experimental rotational constants and dipole moments (determined experimentally through the Stark effect). In Figure 3.8, the calculated structures of the two lowest- 

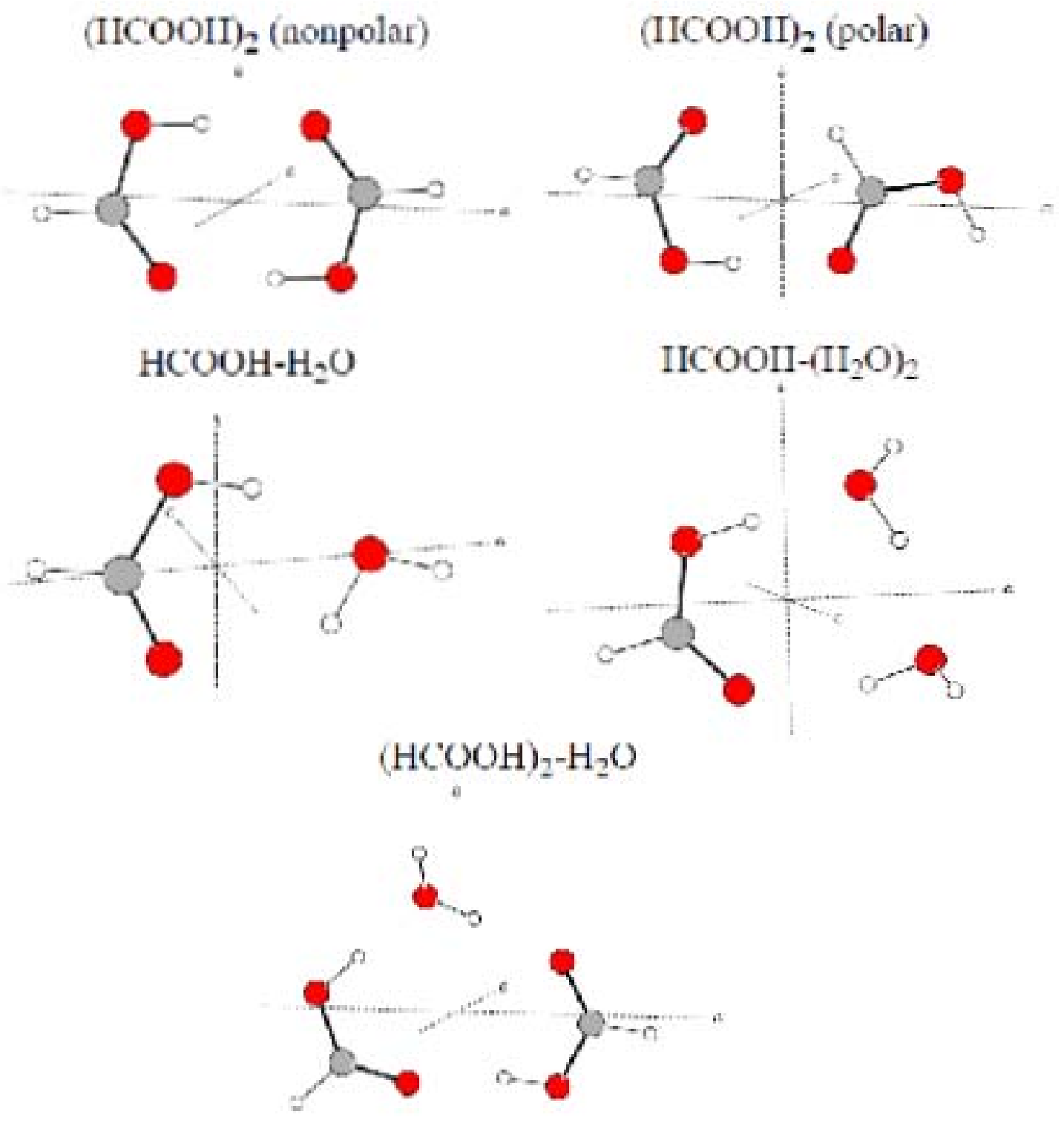

Figure 3.8. Structures of five previously characterized formic acid and formic acid/water complexes, calculated at a MP2/6-31++G(d,p) level of theory. 
energy forms of formic dimer, along with the three complexes from the study of Priem et al., ${ }^{39}$ are shown. It should be noted that formic acid has two conformational isomers, which are referred to as cis or trans by the relative orientation of the two hydrogen atoms; both monomeric units have been characterized by microwave and millimeter-wave spectroscopy, ${ }^{40,41}$ but at room temperature equilibrium, because the trans is more stable by $1365 \mathrm{~cm}^{-1},{ }^{42}$ its population dominates, with a ratio of 700:1 at room temperature. Moreover, the barrier to isomerization between the two isomers is extremely high. ${ }^{43}$ Therefore, in both the complexes that have been previously spectroscopically characterized, as well as the complexes studied here, only the trans conformer of formic acid is observed, and only cluster structures where all of the formic acid monomeric units are in the trans conformation are considered.

In this section, the rotational spectra and structures of three larger complexes of formic acid are presented: $(\mathrm{HCOOH})_{3},(\mathrm{HCOOH})_{3}-\mathrm{H}_{2} \mathrm{O}$, and $(\mathrm{HCOOH})_{5}$. This work builds on a cavity FTMW study of the formic acid trimer by Douglass. ${ }^{44}$ Atom positions derived through isotopic substitution were obtained for all three complexes, in natural abundance $\left({ }^{13} \mathrm{C}\right.$ and $\left.{ }^{18} \mathrm{O}\right)$ for $(\mathrm{HCOOH})_{3}$, and using isotopically labeled samples for $(\mathrm{HCOOH})_{3}-\mathrm{H}_{2} \mathrm{O}$ and $(\mathrm{HCOOH})_{5}$. For both the $(\mathrm{HCOOH})_{3}-\mathrm{H}_{2} \mathrm{O}$ and $(\mathrm{HCOOH})_{5}$ complexes, the spectra were assigned without the guidance of electronic structure theory predictions, and so the identities of the complexes were not conclusively determined until after the spectral assignment of both normal and isotopically substituted species. This project demonstrates the ability of CP-FTMW spectroscopy to drive electronic structure calculations for structure determination, using the tools described earlier in this chapter. 
The experimentally derived structures of each cluster are compared to the results of published theoretical investigations as well as new calculations.

\section{b) Experimental}

Samples of formic acid were purchased from Sigma-Aldrich and used without further purification: $\mathrm{HCOOH}\left(\sim 98 \%\right.$, puriss), $\mathrm{DCOOH}\left(95 \%\right.$ in $\mathrm{H}_{2} \mathrm{O}, 98 \%$ atom D), and $\mathrm{H}^{13} \mathrm{COOH}\left(95 \%\right.$ in $\mathrm{H}_{2} \mathrm{O}, 99 \%$ atom $\left.{ }^{13} \mathrm{C}\right)$. These samples with lower water composition than the typical reagent grade formic acid $(\sim 88 \%)$ are critical to the observation of the complexes presented here; as water readily intercalates into formic acid clusters (a characteristic that is observed in the structures of the observed $(\mathrm{HCOOH})_{2}-\mathrm{H}_{2} \mathrm{O}$ and $(\mathrm{HCOOH})_{3}-\mathrm{H}_{2} \mathrm{O}$ complexes), the presence of too much water greatly diminishes the signal level of the formic acid complexes. Therefore, no water above what is naturally present in the sample was added, except for the addition of a small amount of $\mathrm{H}_{2}{ }^{18} \mathrm{O}$ (98\%, Cambridge Isotopes) in order to measure the spectrum of the $(\mathrm{HCOOH})_{3}-\mathrm{H}_{2}{ }^{18} \mathrm{O}$ complex. For the measurement of singly substituted isotopologues of the formic acid complexes, $\mathrm{DCOOH}$ and $\mathrm{H}^{13} \mathrm{COOH}$ were diluted to roughly $20 \%$ concentration in $\mathrm{HCOOH}$ to maximize the abundance of these species. The ratio of isotopically labeled to normal species formic acid in the expansion was monitored by the ratio of the $3_{12} \leftarrow 3_{13}$ transition of monomeric formic acid and its isotopologues. This is the only transition of $\mathrm{HCOOH}$ with measurable intensity at supersonic expansion temperatures in the frequency ranges probed in this study. 
The sample was placed in a stainless steel reservoir located external to the chamber, over which a carrier gas (either $\mathrm{Ne}$ or $\mathrm{He}$ ) at typically $\sim 3$ atm of backing pressure was flowed. The seeded gas was then expanded through either two or three nozzles. The nozzles were heated to approximately $50{ }^{\circ} \mathrm{C}$, which has the effect of increasing the population of $\mathrm{HCOOH}$ monomer before the supersonic expansion, which is critical for producing large abundances of polar formic acid complexes. Reducedbandwidth CP-FTMW configurations from 7.0-8.8 $\mathrm{GHz}$ and 9.1-13.6 $\mathrm{GHz}$ were employed as described above. For each spectrum, at least $10^{6}$ and up to $4 \times 10^{6}$ FIDs were averaged together.

CP-FTMW Stark spectroscopy, as described above, was used to determine the dipole moment of $(\mathrm{HCOOH})_{3}$. A total of 300,000 FIDs were acquired at a field strength of $240 \mathrm{~V} / \mathrm{cm}$, using OCS as the calibrant. Due to their significantly weaker signals, and the higher $J$ quantum numbers of the transitions visible in the $7.0-18.5 \mathrm{GHz}$ frequency range for these species, the dipole moments of $(\mathrm{HCOOH})_{3}-\mathrm{H}_{2} \mathrm{O}$ and $(\mathrm{HCOOH})_{5}$ could not be determined via the Stark effect and instead the relative magnitudes of their dipole moment components were estimated using relative transition intensities.

\section{c) CP-FTMW spectra}

One of the CP-FTMW spectra used for structural characterization in this study is shown in Figure 3.9. The spectrum is compared to spectral simulations of the three new complexes assigned in this study, using fit Hamiltonian parameters. The left panel shows 

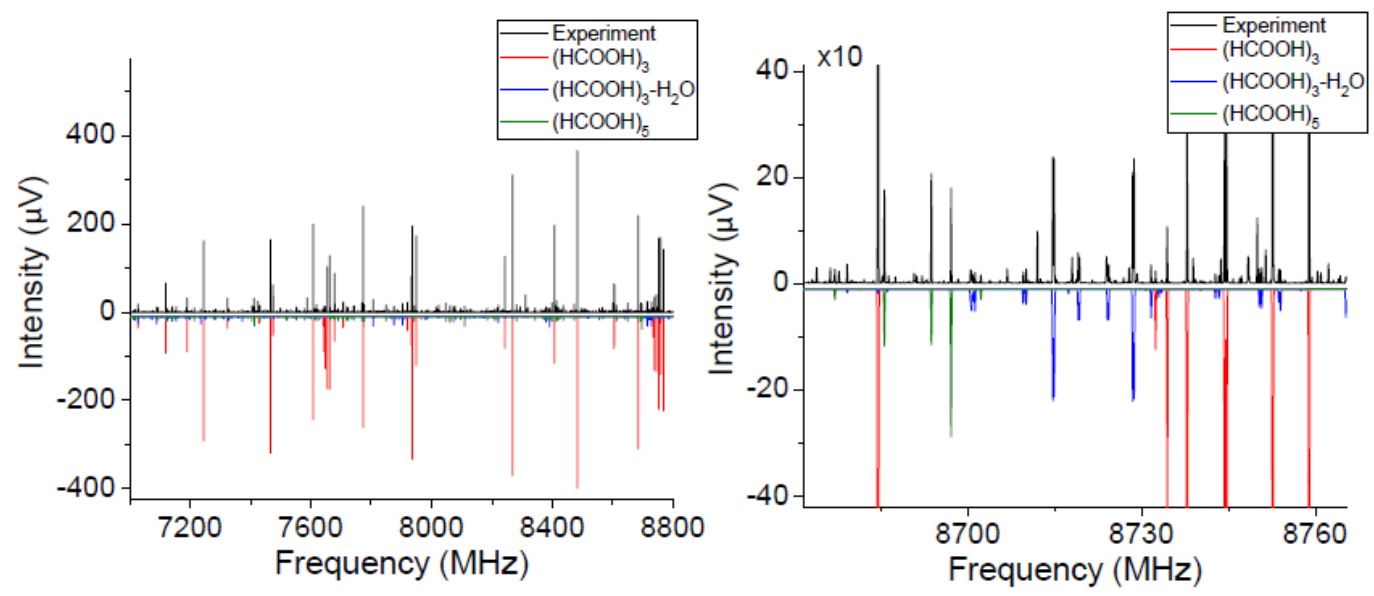

Figure 3.9. The CP-FTMW spectrum of formic acid complexes, using the $7.0-8.8 \mathrm{GHz}$ reduced-bandwidth configuration. A total of 1.981 million FID averages were accumulated for this spectrum. The spectrum is compared to simulations, generated from SPCAT, of the three new complexes assigned in this study (negative-going). The spectrum is dominated by $(\mathrm{HCOOH})_{3}$ (left), while the right panel is an expanded view showing transitions of $(\mathrm{HCOOH})_{5}$ and $(\mathrm{HCOOH})_{3}-\mathrm{H}_{2} \mathrm{O}$. 
that $(\mathrm{HCOOH})_{3}$ is the dominant species in the spectrum. An expanded view in the right panel shows that with the sample conditions described above, both the $(\mathrm{HCOOH})_{3}-\left(\mathrm{H}_{2} \mathrm{O}\right)$ and $(\mathrm{HCOOH})_{5}$ complexes are present at roughly $10 \%$ of the signal level of the formic acid trimer.

As one might expect, the use of isotopically enriched formic acid mixtures greatly enhances the line density of the observed spectrum, which increases the difficulty of spectral assignments, particularly for spectra at low signal-to-noise. To demonstrate the type of spectra that were analyzed for these structural determinations, in Figure 3.10 a portion of the spectrum of ${ }^{13} \mathrm{C}$-enriched formic acid is presented, compared to simulations of the five singly-substituted ${ }^{13} \mathrm{C}$ isotopomers of $(\mathrm{HCOOH})_{5}$. As this figure shows, these spectral analyses offer significant challenges, due to the spectral line density and the number of isotopologues present at the same intensity. However, it should be noted that despite this, the number of blends is relatively low; this is a significant advantage of microwave spectroscopy, as there are an extremely large number of detection channels.

\section{d) Spectrum and structure of $(\mathrm{HCOOH})_{3}$}

Structures of the formic acid trimer have been studied theoretically. ${ }^{45,46}$ In both of these studies the lowest-energy isomer of this complex was calculated to be a nonpolar formic acid dimer, as seen in Figure 3.8, with a third monomer unit attached with its third hydroxyl proton donating a hydrogen bond to a carbonyl oxygen of the dimer unit. This is more stable than donating a hydrogen bond to the hydroxyl group oxygen by roughly 


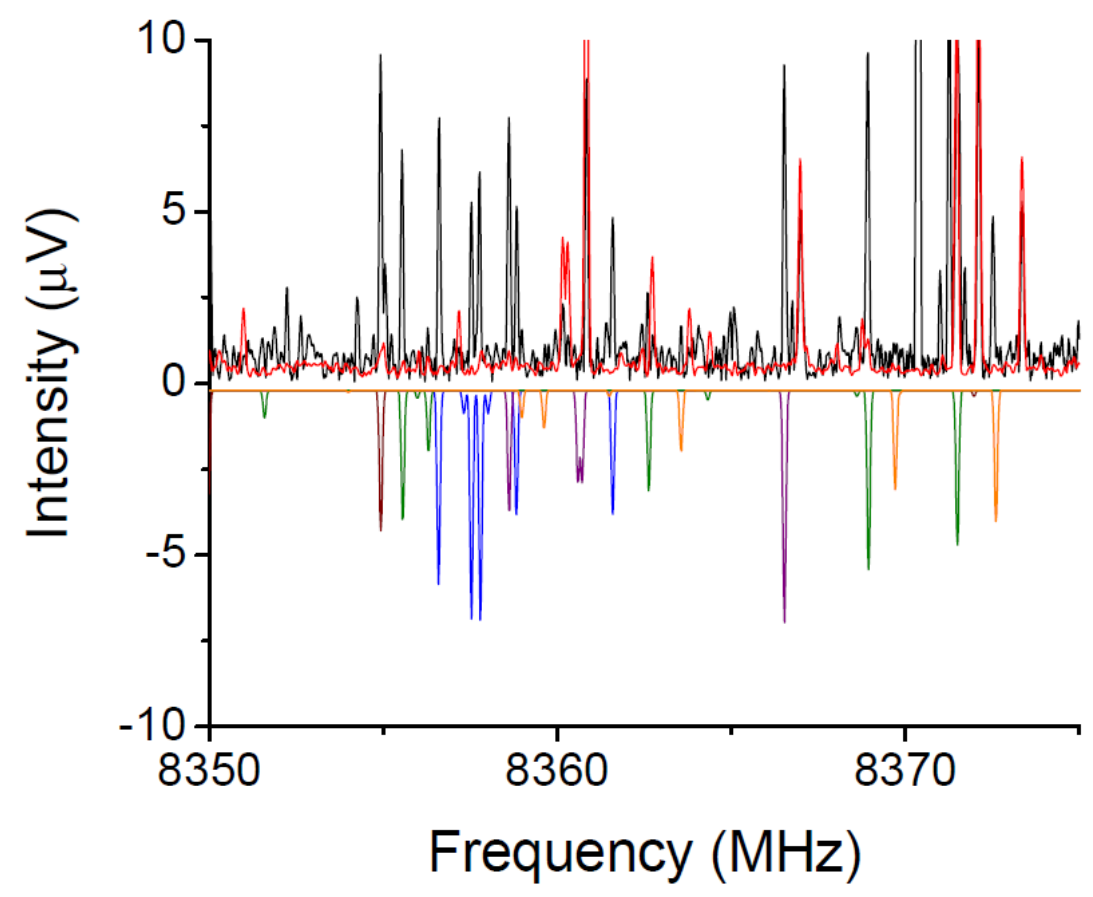

Figure 3.10. A portion of the CP-FTMW spectrum of ${ }^{13} \mathrm{C}$-enriched (20\%) formic acid (black) compared to the spectrum of formic acid with no isotopic enrichment (red). The colored negative-going traces are simulations of the five singly-substituted ${ }^{13} \mathrm{C}$ isotopologues of $(\mathrm{HCOOH})_{5}$. 
$700 \mathrm{~cm}^{-1}$. The second-lowest isomer of formic acid is a cyclic trimer structure that is nonpolar, and therefore invisible in rotational spectroscopy studies. In the previous experimental study of the formic acid trimer, the structure of the experimentally observed isomer was identified based on the agreement between experimental and calculated rotational constants. The next highest polar isomer, despite being different from the lower-energy isomer only by exchanging the two hydroxyl protons between the subunits of the dimer, has considerably different calculated rotational constants, and therefore a reasonably confident assignment can be made from this agreement alone. The fit Hamiltonian parameters derived from the measured CP-FTMW spectra in this study are presented in Table 3.5, compared to calculated parameters using both density functional theory (B3LYP) and MP2 methods. It can be seen from Table 3.5 that the B3LYP calculated rotational constants and dipole moments agree with experiment more closely than the MP2 calculated constants.

As further confirmation of this assignment, and to explore the accuracy of the calculated structures, experimental structures were derived for the observed isomer of $(\mathrm{HCOOH})_{3}$. A total of 16 minor isotopologues of this isomer were assigned: three singly-substituted ${ }^{13} \mathrm{C}$ isotopologues, six singly-substituted ${ }^{18} \mathrm{O}$ isotopologues, three singly-substitued deuterated isotopologues (for the $\mathrm{C}-\mathrm{H}$ protons), as well as three doubly${ }^{13} \mathrm{C}$ substituted species and the triply ${ }^{13} \mathrm{C}$-substitued species. It should be noted that the multiply-substituted isotopologues provide minimal additional structural information, but were measured as byproducts of the ${ }^{13} \mathrm{C}$-enriched measurements more targeted at 
Table 3.5. Fit $A$-reduction Hamiltonian parameters of $(\mathrm{HCOOH})_{3}$, compared to values calculated by electronic structure theory.

\begin{tabular}{cccc}
\hline & & \multicolumn{2}{c}{ Ab Initio } \\
Parameter & Experiment & B3LYP/ & MP2/ \\
& $2936.5116(4)$ & 2877.50 & $6-311++\mathrm{G}(\mathrm{d}, \mathrm{p})$ \\
\hline$A(\mathrm{MHz})$ & $595.07077(7)$ & 595.28 & 603.28 \\
$B(\mathrm{MHz})$ & $495.25989(6)$ & 493.24 & 495.689 \\
$C(\mathrm{MHz})$ & $0.07676(24)$ & & \\
$\Delta_{\mathrm{J}}(\mathrm{kHz})$ & $-0.2838(9)$ & & \\
$\Delta_{\mathrm{JK}}(\mathrm{kHz})$ & $4.56(3)$ & & \\
$\Delta_{\mathrm{K}}(\mathrm{kHz})$ & $0.01674(6)$ & & \\
$\delta_{\mathrm{J}}(\mathrm{kHz})$ & $0.293(4)$ & & \\
$\delta_{\mathrm{K}}(\mathrm{kHz})$ & $1.18(6)$ & 1.43 & 1.36 \\
$\mu_{\mathrm{A}}(\mathrm{debye})$ & $0.995(12)$ & 1.13 & \\
$\mu_{\mathrm{B}}($ debye $)$ & $1.54(5)$ & 1.82 & 52.3 \\
$\mu_{\text {total }}($ debye $)$ & $40.1(15)$ & 38.3 & 0.004 \\
$\theta(\text { degrees })^{\mathrm{a}}$ & -0.945 & 0.003 & \\
$\Delta_{\left(\mathrm{u} \AA^{2}\right)^{\mathrm{b}}}$ & 136 & & \\
$\mathrm{~N}_{\text {lines }}$ & 3.1 & & \\
r.m.s. error $(\mathrm{kHz})$ & & & \\
\hline
\end{tabular}

${ }^{\mathrm{a}}$ Angle between the dipole moment vector and the $a$ principal axis.

${ }^{\mathrm{b}}$ Inertial defect, defined as $I_{\mathrm{c}}-I_{\mathrm{a}}-I_{\mathrm{b}}$. 
understanding the structures of larger clusters. From this isotopic information, two experimental structures were derived. The first was derived using Kraitchman's equations, where the position of a given atom is derived from the change in the rotational constants of the complex on the isotopic substitution of that atom, while the second was derived from a least-squares fit of all of the experimental moments of inertia of all of the assigned isotopologues to 21 internal coordinates of the complex. In the least-squares fit, the complex was forced to be planar; as seen in Table 3.5, the complex does have a small, negative inertial defect, signifying slight non-planarity, due to out-of-plane zero-point vibrational motion. Additionally, because no isotopic information was derived for the hydroxyl protons, their positions were not fit in this analysis, and their bond lengths and bond angles were held fixed to ab initio values calculated at a B3LYP/6-31G(d,p) level of theory.

Figure 3.11 shows the two experimentally derived structures of $(\mathrm{HCOOH})_{3}$ compared to B3LYP and MP2 calculated structures. The two experimental structures agree extremely closely, with the exception of the carbon and carbonyl oxygen atoms ofthe central monomer unit. In the Kraitchman analysis, the derived $a$ coordinate of these two atoms is imaginary, and therefore in the displayed structures is constrained to zero. In the least-squares optimized structure, the positions of these two atoms are more in agreement with the calculated structures; other than these two atoms, the structure is nearly identical. The r.m.s. deviation between theory and experiment (using the Kraitchman-derived atom positions) for the B 3 LYP structure is $0.064 \AA$, compared to 


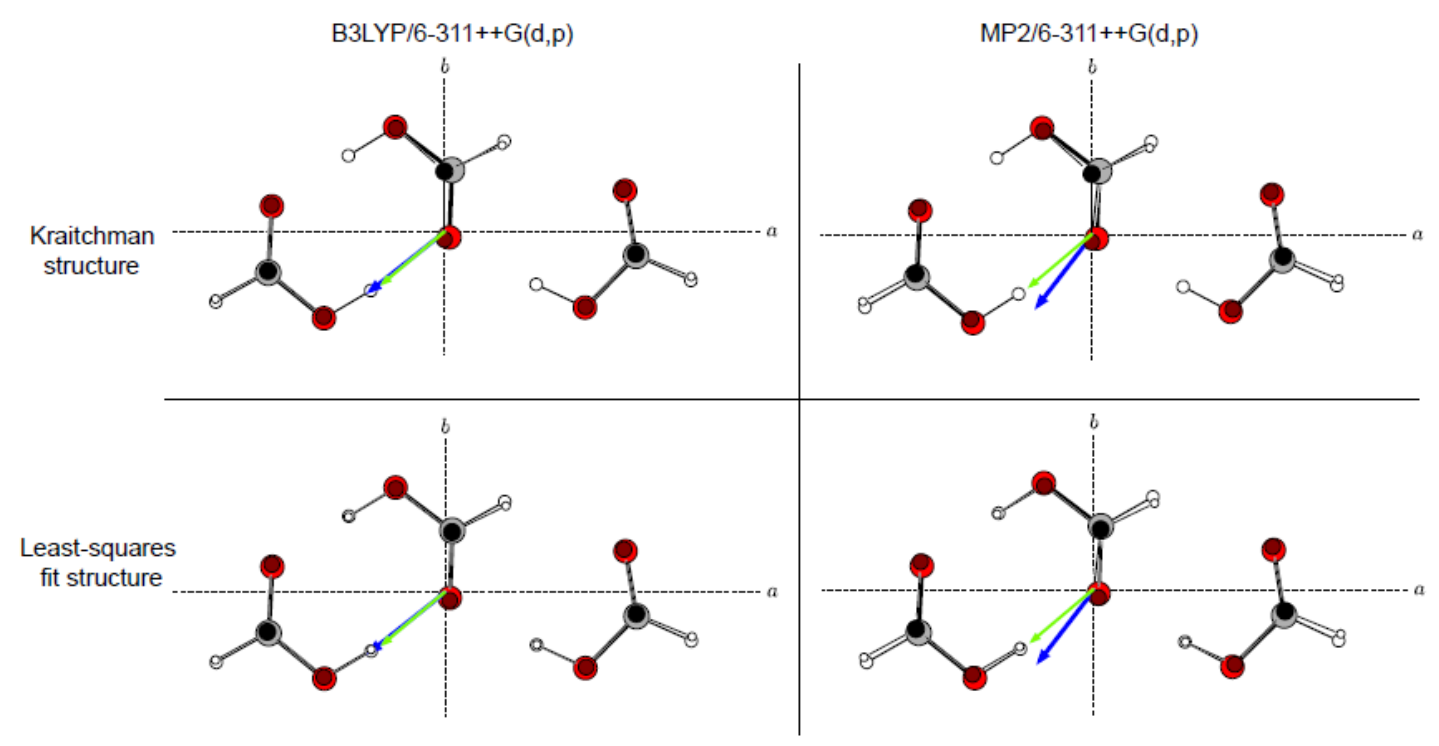

Figure 3.11. Structure of the observed $(\mathrm{HCOOH})_{3}$ complex. In each panel, the smaller circles are experimentally derived atom positions, using either the Kraitchman or leastsquares techniques, while the larger circles are $a b$ initio structures calculated at a B3LYP/6-311++G(d,p) level of theory or an MP2/6-311++G(d,p) level of theory (bottom frame). The calculated dipole moments are shown as blue arrows, while the experimentally determined dipole moment is shown as a green arrow. 
$0.090 \AA$ for the MP2 structure. When the two atoms that lie near to the $a$ principal axis are neglected, the deviations drop to $0.043 \AA$ for the B3LYP structure and $0.076 \AA$ for the MP2 structure.

The most significant discrepancy between the B3LYP and MP2 calculations is in the orientation of the dipole moment vector. In Figure 3.11, the calculated dipole moment is shown as a thicker blue arrow, while the experimentally derived dipole moment is a thinner green arrow. The length of the vector corresponds to the magnitude of the dipole moment. The experimental dipole moment was derived by a fit to 12 Starkshifted transitions ( $4 a$-type and $8 b$-type). The angle between the dipole moment and the $a$ axis derived via the Stark effect $\left(38.8^{\circ}\right)$ is in reasonably close agreement with the angle derived from the relative intensities of $a$ and $b$-type transitions $\left(41^{\circ}\right)$. The B3LYP calculated angle is close to experiment, but the MP2 calculation is in significant deviation, as can be seen in Figure 3.11 and Table 3.5. This is a highly peculiar result that is not fully understood. As seen in the strawberry aldehyde study above, and particularly in Table 3.2, with only a few exceptions, the dipole moment was fairly invariant to the theoretical method employed, even though strawberry aldehyde is significantly larger than $(\mathrm{HCOOH})_{3}$. It should be noted that the small structural differences discussed above between the MP2 and B3LYP calculations are not responsible for the dipole moment rotation between the two calculations: the dipole moment of the MP2 optimized structure, calculated at a B3LYP level of theory, is more in agreement with the observed dipole moment (with $\mu_{\mathrm{a}}>\mu_{\mathrm{b}}$ ). The error, therefore, must be in the calculation of the induced dipole moments between the three formic acid units. 
As discussed above, the observed isomer of $(\mathrm{HCOOH})_{3}$ can be thought of as a complex between the nonpolar formic acid dimer and another $\mathrm{HCOOH}$ unit. Therefore, to a first approximation, the dipole moment of the formic acid trimer should be approximately that of the formic acid monomer (for which $\mu=1.415 \mathrm{D}$ ), ${ }^{47}$ since the formic acid dimer is nonpolar. As seen in Table 3.5, the magnitude of the experimentally derived dipole moment is reasonably close to this value. A method for calculating the induced dipole moment in molecular complexes has been described, ${ }^{48,49}$ and more thorough theoretical work will be needed to explore the nature of the deviation between the experimental and MP2 calculated dipole moment.

e) Spectrum and structure of $(\mathrm{HCOOH})_{3}-\mathrm{H}_{2} \mathrm{O}$

To the author's knowledge, only one computational study of the $(\mathrm{HCOOH})_{3}-\mathrm{H}_{2} \mathrm{O}$ complex has been published. ${ }^{50}$ For this complex, the observed signal to noise ratio was lower than for formic acid trimer, as can be seen in Figure 3.9, and so the ${ }^{13} \mathrm{C}$ and ${ }^{18} \mathrm{O}$ isotopologues could not be measured in natural abundance. However, isotopic information could still be obtained using isotopically enriched samples. The positions of the three carbon atoms, the three hydrogens bonded to carbon atoms, and the water oxygen atom were determined. The calculated structure of the assigned isomer of $(\mathrm{HCOOH})_{3}-\mathrm{H}_{2} \mathrm{O}$, overlaid with the experimentally derived substitution coordinates, is presented in Figure 3.12. The water molecule is intercalated into the formic acid trimer, rather than attaching to it, so that every hydroxyl proton in the complex donates a 

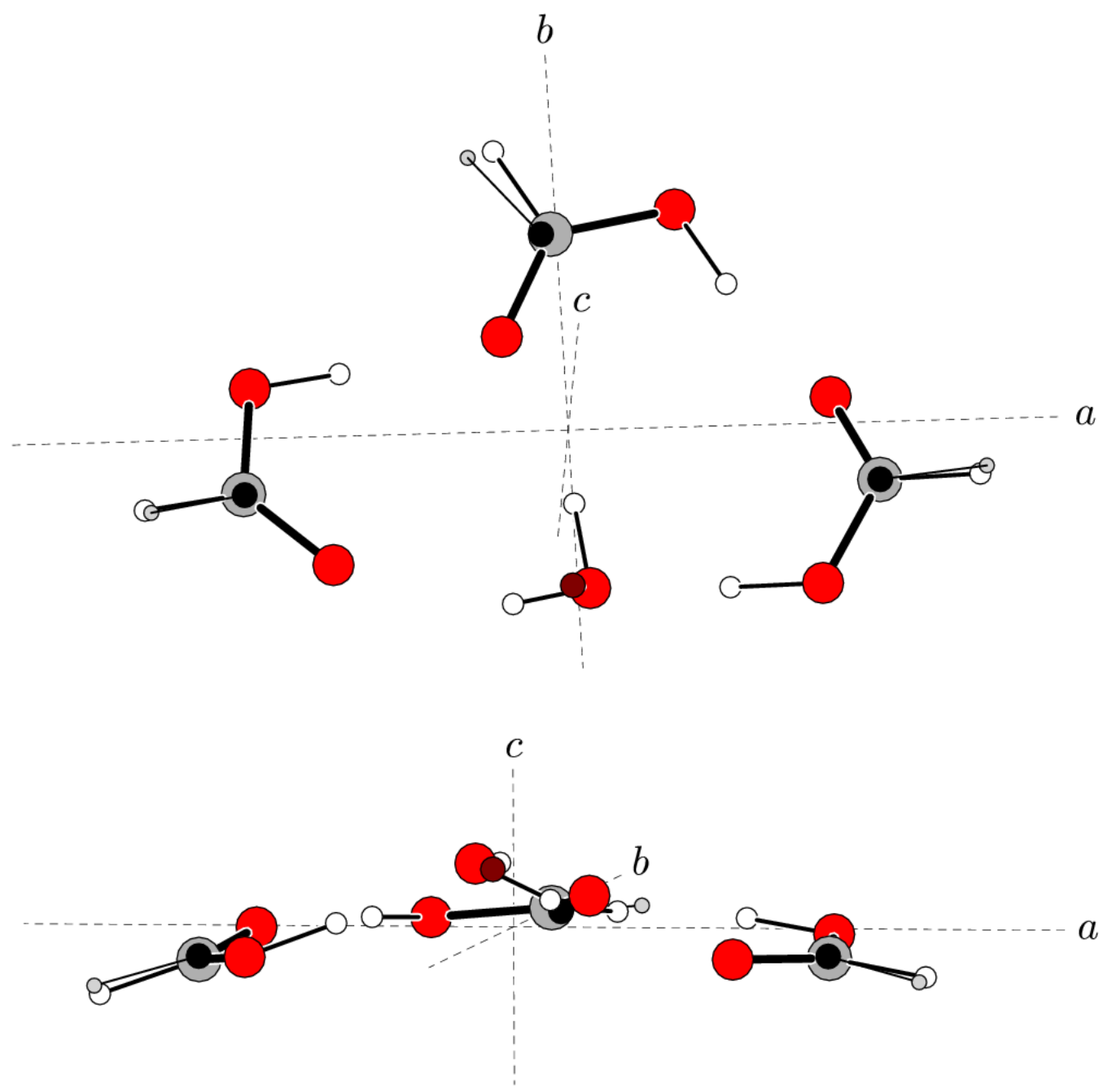

Figure 3.12. Two views of the structure of the experimentally observed $(\mathrm{HCOOH})_{3^{-}}$ $\left(\mathrm{H}_{2} \mathrm{O}\right)$ complex. The smaller circles indicate experimental atom positions as determined through a Kraitchman analysis, while the larger circles indicate the ab initio structure at an MP2/6-31++G(d,p) level of theory. 
hydrogen bond to another oxygen. Our theoretical studies found that water could not add to the planar formic acid trimer so that this could be the case, and in fact all geometry optimizations beginning with water adding to a planar formic acid trimer structure led to breaking of the planarity of the trimer. The water/formic interactions, therefore, appear to be strong enough to dramatically disrupt the structure of the complex.

It should be noted that this structure is very similar to that of the $(\mathrm{HCOOH})_{2}-\mathrm{H}_{2} \mathrm{O}$ complex, previously characterized by Priem et al., ${ }^{39}$ with a third formic acid unit attached. The root-mean-square deviation between the calculated and experimental atom positions is $0.153 \AA$. This structure is similar to the minimum-energy structure calculated by Allouche, ${ }^{50}$ except the structure calculated in this study has one of the water protons oriented out of the plane of the complex rather than towards the carbonyl oxygen across the complex (the water $\mathrm{O}-\mathrm{H}$ bond that is nearly aligned with the $b$ principal axis). The structure presented in Figure 3.12 is lower in energy than the structure reported by Allouche by $86 \mathrm{~cm}^{-1}$ (calculated at an MP2/6-31++G(d,p) level of theory); additionally, the structure of Allouche is far more puckered than that presented in Figure 3.12, and so has markedly different atom positions, rotational constants, and dipole moment components from that of the experimentally observed complex. Therefore the evidence is strong that the structure presented in Figure 3.12 is that of the experimentally observed complex.

Figure 3.12 shows that, additionally, this complex is significantly nonplanar. All transitions of all eight assigned isotopomers of this complex were found to be split into two lines of equal intensity. This splitting is attributed to low-barrier tunneling between 
equivalent nonplanar structures through a planar transition state, as seen in Figure 3.13. This tunneling is equivalent to a reflection about the $c$ principal axis of the complex. Because of this tunneling, the eigenstates of the molecular Hamiltonian are symmetric and antisymmetric combinations of the two equivalent nonplanar structures; these states are labeled $0^{+}$(for the symmetric combination) and $0^{-}$(for the antisymmetric combination), with the symmetric state being lower in energy. The states have different rotational constants, because their vibrational wavefunctions are slightly different. For $a$ and $b$-type transitions, the vibrational selection rules require either $0^{+} \leftarrow 0^{+}$or $0^{-} \leftarrow 0^{-}$, so the transitions corresponding to the two torsional substates are very close in frequency due to the similar rotational constants between the two species. However, for $c$-type transitions, because the tunneling motion involves a reflection about this axis, the torsional selection rules are $0^{+} \leftarrow 0^{-}$or $0^{-} \leftarrow 0^{+}$. Therefore, transitions are split by twice the energy gap between the two species. It should be noted that the $0^{-}$state, as shown in Table 3.6, has a slightly higher inertial defect (the deviation of the structure from planarity). This is expected as the $0^{-}$state, being higher in energy, exhibits larger zeropoint vibrational excursions from the equilibrium geometry than the $0^{+}$state, and therefore would be expected to be more nonplanar. The $c$-type transitions of the complex were initially found using FTMW-MW double resonance spectroscopy in a BalleFlygare-type cavity spectrometer. For this experiment, a $b$-type transition was monitored, and the double resonance source was scanned beginning at the frequency of a connected $c$-type transition under rigid rotor selection rules (without the tunneling gap). Once a 


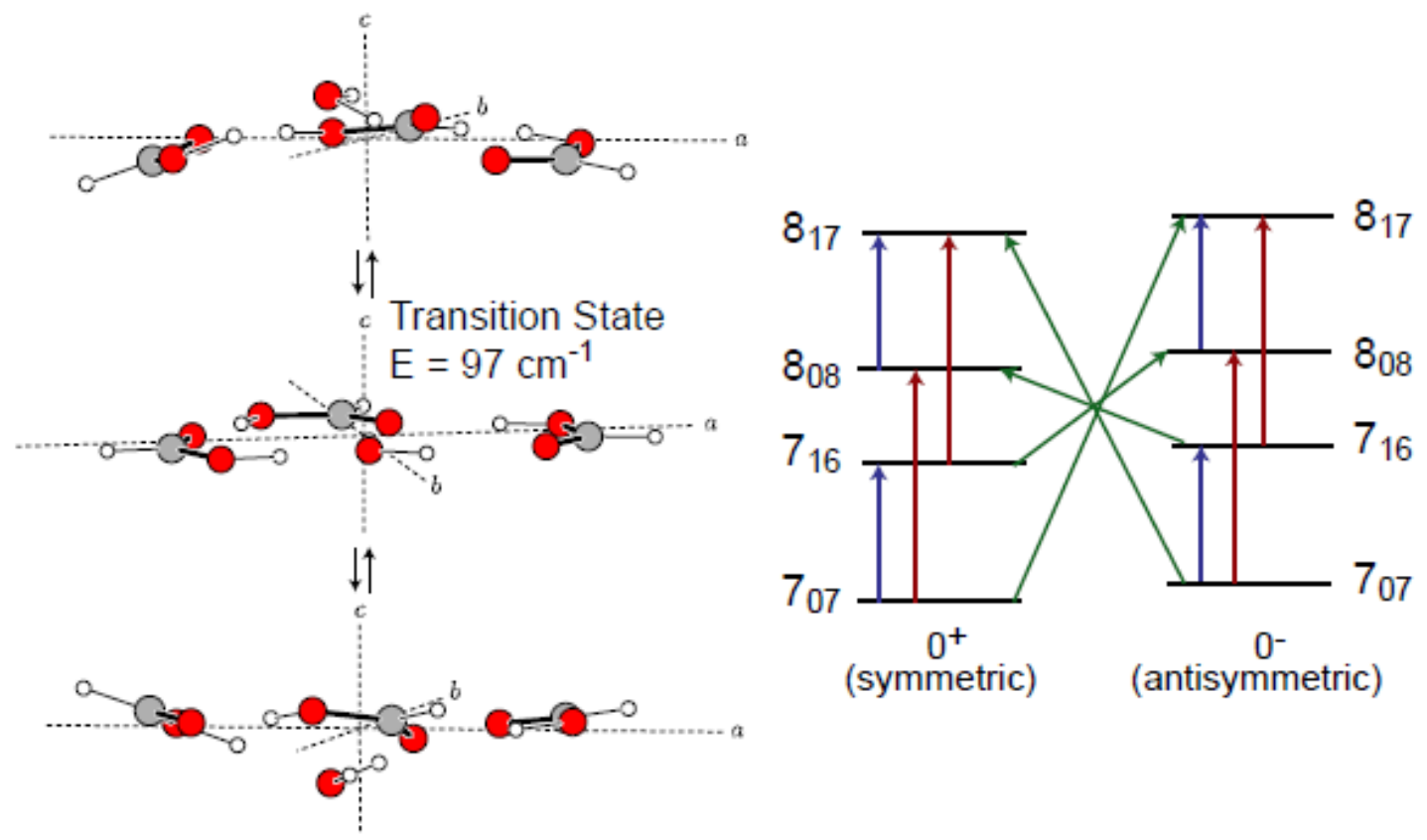

Figure 3.13. Tunneling in the $(\mathrm{HCOOH})_{3}-\left(\mathrm{H}_{2} \mathrm{O}\right)$ complex. Left: the two equivalent minimum energy structures and the planar transition state. On the right, rotational selection rules in the complex are demonstrated: transitions with $a$-type (red arrows) and $b$-type (blue arrows) selection rules require $0^{+} \rightarrow 0^{+}$or $0^{-} \rightarrow 0^{-}$, while the $c$-type transitions (green arrows) require $0^{+} \rightarrow 0^{-}$or $0^{-} \rightarrow 0^{+}$. 
Table 3.6. Fit $A$-reduction Hamiltonian parameters of $(\mathrm{HCOOH})_{3}-\left(\mathrm{H}_{2} \mathrm{O}\right)$, compared to values calculated by electronic structure theory.

\begin{tabular}{|c|c|c|c|}
\hline \multirow[b]{2}{*}{ Parameter } & \multicolumn{2}{|c|}{ Experiment } & \multirow{2}{*}{$\begin{array}{c}A b \text { Initio } \\
\mathrm{MP} 2 / 6-31++\mathrm{G}(\mathrm{d}, \mathrm{p})\end{array}$} \\
\hline & $0^{+}$state & $0^{-}$state & \\
\hline$A(\mathrm{MHz})$ & $1326.6161(4)$ & $1326.5814(4)$ & 1299.64 \\
\hline$B(\mathrm{MHz})$ & $588.85651(18)$ & $588.87434(19)$ & 587.34 \\
\hline$C(\mathrm{MHz})$ & $416.19911(21)$ & $416.21038(21)$ & 414.43 \\
\hline$\Delta_{\mathrm{J}}(\mathrm{kHz})$ & \multicolumn{2}{|c|}{$0.1654(7)$} & $0.199^{\mathrm{a}}$ \\
\hline$\Delta_{\mathrm{JK}}(\mathrm{kHz})$ & \multicolumn{2}{|c|}{$-0.498(4)$} & $-0.930^{\mathrm{a}}$ \\
\hline$\Delta_{\mathrm{K}}(\mathrm{kHz})$ & \multicolumn{2}{|c|}{$1.676(10)$} & $2.141^{\mathrm{a}}$ \\
\hline$\delta_{\mathrm{J}}(\mathrm{kHz})$ & \multicolumn{2}{|c|}{$0.4621(27)$} & $0.0577^{\mathrm{a}}$ \\
\hline$\delta_{\mathrm{K}}(\mathrm{kHz})$ & \multicolumn{2}{|c|}{$0.217(5)$} & $0.109^{\mathrm{a}}$ \\
\hline$\Delta E(\mathrm{MHz})$ & \multicolumn{2}{|c|}{$178.8329(3)$} & \\
\hline$F_{a}(\mathrm{MHz})$ & \multicolumn{2}{|c|}{$0.458(9)$} & \\
\hline$\mu_{\mathrm{A}}($ debye $)$ & & & 1.03 \\
\hline$\mu_{\mathrm{B}}($ debye $)$ & & & 2.36 \\
\hline$\mu_{\mathrm{C}}($ debye $)$ & & & 1.18 \\
\hline$\mu_{\text {total }}($ debye $)$ & & & 2.83 \\
\hline$\left(\mu_{\mathrm{B}} / \mu_{\mathrm{A}}\right)^{2}$ & \multicolumn{2}{|c|}{5.0} & 5.29 \\
\hline$\left(\mu_{\mathrm{B}} / \mu_{\mathrm{C}}\right)^{2}$ & \multicolumn{2}{|c|}{4.2} & 4.00 \\
\hline$\Delta\left(\mathrm{u} \AA^{2}\right)^{\mathrm{a}}$ & -24.919 & -24.936 & -29.86 \\
\hline $\mathrm{N}_{\text {lines }}$ & \multicolumn{2}{|c|}{315} & \\
\hline r.m.s. error (kHz) & \multicolumn{2}{|c|}{15.9} & \\
\hline
\end{tabular}

${ }^{a}$ Centrifugal distortion constants were calculated at a B3LYP/6-31++G(d,p) level of theory (using the MP2/6-31++G(d,p) optimized structure).

${ }^{\mathrm{b}}$ Inertial defect, defined as $I_{\mathrm{c}}-I_{\mathrm{a}}-I_{\mathrm{b}}$. 
connected $c$-type transition was found, a few other transition frequencies predicted by the gap derived from this transition were checked for resonance. Based on this data, a large number of $c$-type transitions were then found in the high-sensitivity CP-FTMW spectrum, leading to a precise determination of the tunneling gap.

An analysis of the rotational spectrum requires the simultaneous fitting of the two states; the fit Hamiltonian parameters resulting from this analysis are presented in Table 3.6. The form of the Hamiltonian model is

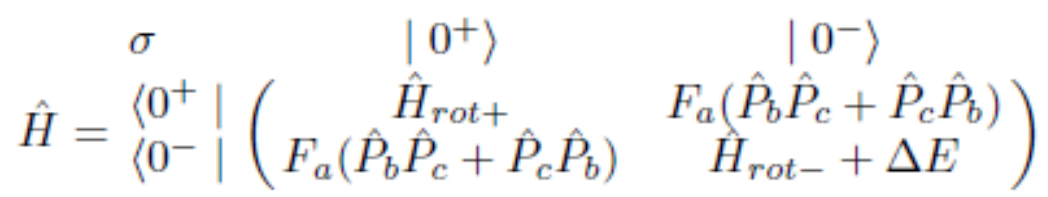

where $H_{\text {rot }}$ is the standard rotational Hamiltonian with Watson $A$-reduction quartic centrigual distortion constants, $\Delta E$ is the energy splitting between the two torsional substates, and $F_{\mathrm{a}}$ is a Coriolis term that represents the vibration-rotation coupling due to the tunneling motion; that is, the tunneling motion induces rotation about the $a$ axis. It should be noted that effect of this axis-switching term is small, as a reasonably good fit can be achieved without its inclusion; however, because this constant is well determined, and it significantly lowers the r.m.s. deviation between experimental and calculated transition frequencies, it is included in the analysis of Table 3.6. However, because of reduced signal-to-noise ratio, $c$-type transitions were not observed for the isotopologues, so $\Delta E$ could not be directly determined. Therefore, the Coriolis term was not included in these fits, as it was poorly constrained without transitions crossing the tunneling gap. For the isotopically substituted species, therefore, the spectrum was fit to a two-state 
Hamiltonian without interacting terms. For all isotopologues, the centrifugal distortion constants were constrained to be the same between the two torsional substates.

The tunneling splitting can be theoretically calculated using a simple, onedimensional WKB model assuming an Eckart symmetric well potential. This technique has been previously applied in spectroscopic studies of malonaldehyde ${ }^{51}$ and 1,3propanediol. $^{52}$ The energy splitting between the two torsional substates is calculated as

$$
\Delta E=\frac{h v_{f}}{\pi} e^{-\theta}
$$

where $v_{\mathrm{f}}$ is the vibrational frequency of the inversion at the minimum energy structure (calculated to be $25.6 \mathrm{~cm}^{-1}$ ) and $\theta$ is the penetration integral, given by

$$
\theta=\frac{2 \pi}{h v_{i}}\left(V_{e f f}-\sqrt{\frac{1}{2} h v_{f} V_{e f f}}\right)
$$

with $V_{\text {eff }}$ representing the barrier to interconversion between the two equivalent species (with zero-point vibrational energy corrections included for all modes except the inversion mode), calculated to be $97.4 \mathrm{~cm}^{-1}$, and $v_{\mathrm{i}}$ is the imaginary vibrational frequency of the inversion mode at the transition state, calculated to be $44.7 i \mathrm{~cm}^{-1}$. With these parameters, an energy splitting of $39.9 \mathrm{MHz}$ is calculated. For computational speed, the vibrational frequencies of the minimum and transition state structures were calculated at a B3LYP/6-31G++(d,p) level of theory. While this appears to be in significant deviation from the experimentally observed value of $178.8329(3) \mathrm{MHz}$, the exponential dependence of the tunneling splitting on the energy gap means that small changes in the barrier and the vibrational frequency of the inversion mode have large effects on this splitting. If the barrier in the model is reduced to $84 \mathrm{~cm}^{-1}$ (a reduction of only $13 \%$, well 
within the error of the calculation), the calculated tunneling splitting matches the experimentally observed value. Alternatively, an increase in the vibrational frequencies of the inversion mode in both the minimum and transition state by $15 \%$ reproduces the experimentally observed value. Therefore, using this simple model, the observed tunneling splitting is in qualitative agreement with the proposed internal motion of the complex.

Because of the lower signal-to-noise ratio of the $(\mathrm{HCOOH})_{3}-\mathrm{H}_{2} \mathrm{O}$ complex, as well as the higher $J$ quantum number of the transitions observed in the frequency range of this experiment, the dipole moment of this complex could not be determined through the Stark effect. Therefore, the relative dipole moment components were estimated through the relative intensities of $a, b$, and $c$ type transitions in this normal species, as in the strawberry aldehyde study. The observed relative transition intensities are estimated to be 1:5:1.2 (a-type: $b$-type: $c$-type). In Table 3.6 , it can be seen that these relative dipole moment components are in very good agreement with the ratios calculated by electronic structure theory.

f) Spectrum and structure of $(\mathrm{HCOOH})_{5}$

After the trimer, the next two largest pure formic acid clusters, $(\mathrm{HCOOH})_{4}$ and $(\mathrm{HCOOH})_{5}$, are fundamentally different in that $\pi$-stacking interactions can begin to play a role in the structure of these complexes. As discussed above in the strawberry aldehyde section, these weaker interactions can be harder to quantify using theoretical methods. A study of the formic acid tetramer using the B3LYP functional by Roy and Thakkar found 
a planar minimum-energy structure, with two nonpolar dimer linked by two C-H...O interactions, ${ }^{53}$ but two more recent studies using MP2 theory as well as other density functional methods ${ }^{54,55}$ calculated that the minimum energy structure consists of two $\pi$ stacked nonpolar dimers. Due to the symmetry of $(\mathrm{HCOOH})_{4}$, the calculated minimum energy structures (both planar and stacked) have no dipole moment and so cannot be measured using pure rotational spectroscopy. Therefore, the next largest formic acid cluster that can be studied by microwave spectroscopy is the formic acid pentamer.

Structures of the formic acid pentamer have been calculated by Roy and Thakkar, ${ }^{56}$ also using the B3LYP functional as in their formic acid tetramer study. Therefore, an experimentally derived structure for this complex can help assess whether B3LYP is truly underestimating these stacking interactions. The formic acid tetramer is also far more challenging than the previous two clusters described above because the number of possible isomers is significantly greater: a total of 205 local minimum structures were calculated at the B3LYP/pc2 level of theory in the previous study. A large number of structurally diverse isomers (47) were found in this study to be reasonably close in energy (within $\sim 1000 \mathrm{~cm}^{-1}$ ). It should be noted that this study also included minima in which one or more of the subunits is in its cis conformation, but because of the low population of cis-formic acid anticipated in the supersonic expansion as discussed above, only isomers in which all of the monomers are trans are considered.

Based on a series of characteristic $a-b$ quartets, a spectrum was assigned from the deep-average CP-FTMW spectra that was found to be both very nonplanar (inertial defect -543.7 $\mathrm{u} \AA^{2}$ ) and chiral (that is, $\mu_{a}, \mu_{b}$, and $\mu_{c}$ are each known to be nonzero due to the 
observation of all three types of transitions), fit with a normal Watson $A$-reduction Hamiltonian with quartic distortion constants. No structure due to internal motions was observed. Because these characteristics of this structure disagreed with those anticipated by the previous theoretical study, the assignment of the observed spectrum to the formic acid pentamer was tentative until the assignment of five singly-substituted ${ }^{13} \mathrm{C}$ isotopologues and five singly-substituted D isotopologues from the isotopically enriched samples as described above. Once the observed spectrum was confirmed to be formic acid pentamer using this information, other possible structures that would be consistent with this experimentally derived structural information were explored.

Even with the substitution coordinates of ten atoms determined in this way, a precise structural determination of the observed formic acid pentamer complex is challenging. First, isotopic substitution information only provides information about the magnitude of the coordinates of each substituted atom, so there are eight possible locations for each substituted atom. Because the overall signs of the coordinates of the structure are arbitrary, this means that for $N$ atom positions, there are in general a total of $2^{3(N-1)}$ possible relative atomic orientations, which for formic acid pentamer (with 10 substituted atoms) would mean there are $1.34 \times 10^{8}$ structures that must be considered with this information. However, these candidate orientations were narrowed significantly using atomic connectivity information. The first constraint that can be applied is that, because each hydrogen atom must be bonded to a carbon atom, the bond length should be close to that of the formic acid monomer $(\sim 1.1 \AA)$. From this information, each hydrogen could be readily paired with the carbon atom from the same subunit. Four of the five C- 
$\mathrm{H}$ bond lengths were very close to this value (within $\sim 0.2 \AA$ ), with each of the hydrogen atoms located in the same octant as its companion carbon atom; for the fifth, the bond length assuming that the C-H bond is in one octant is an unphysical $0.496 \AA$; this bond more likely crosses the $a$ principal axis of the complex, in which case the $\mathrm{C}-\mathrm{H}$ bond length is $0.708 \AA$, which is still unphysically short, but the small $a$ coordinates of the two atoms increases the uncertainty on the atom positions, which could explain this deviation.

With this information, the number of possible structures is reduced to $2^{12}=4096$ possible structures. This number is further reduced by imposing restrictions on the possible $\mathrm{C}-\mathrm{C}$ distances in the formic acid complex. In the nonpolar formic acid dimer, this separation is $3.86 \AA$. Based on initial calculations, the C-C separation could decrease from this in isomers where formic acid subunits are "stacked," to a minimum of $\sim 3 \AA$. Of the 4096 possible structures, only $32\left(2^{5}\right)$ satisfy the requirement that each of the C-C separations be greater than $3 \AA$, representing five atomic coordinates that could be inverted and still satisfy this requirement. Of these five coordinates, four are fairly small in magnitude $(<0.6 \AA)$ and therefore do not represent dramatic qualitative changes in the structure of the complex. The fifth, however, with magnitude $2.40 \AA$, differentiates the structures into two families.

A number of optimized structures have been calculated based on the two possible structural families. The most likely structure of $(\mathrm{HCOOH})_{5}$ calculated in this study is presented in Figure 3.14. This structure consists of roughly a nonpolar dimer stacked on a trimer in the configuration assigned in this study. The subunits are very nearly planar, 

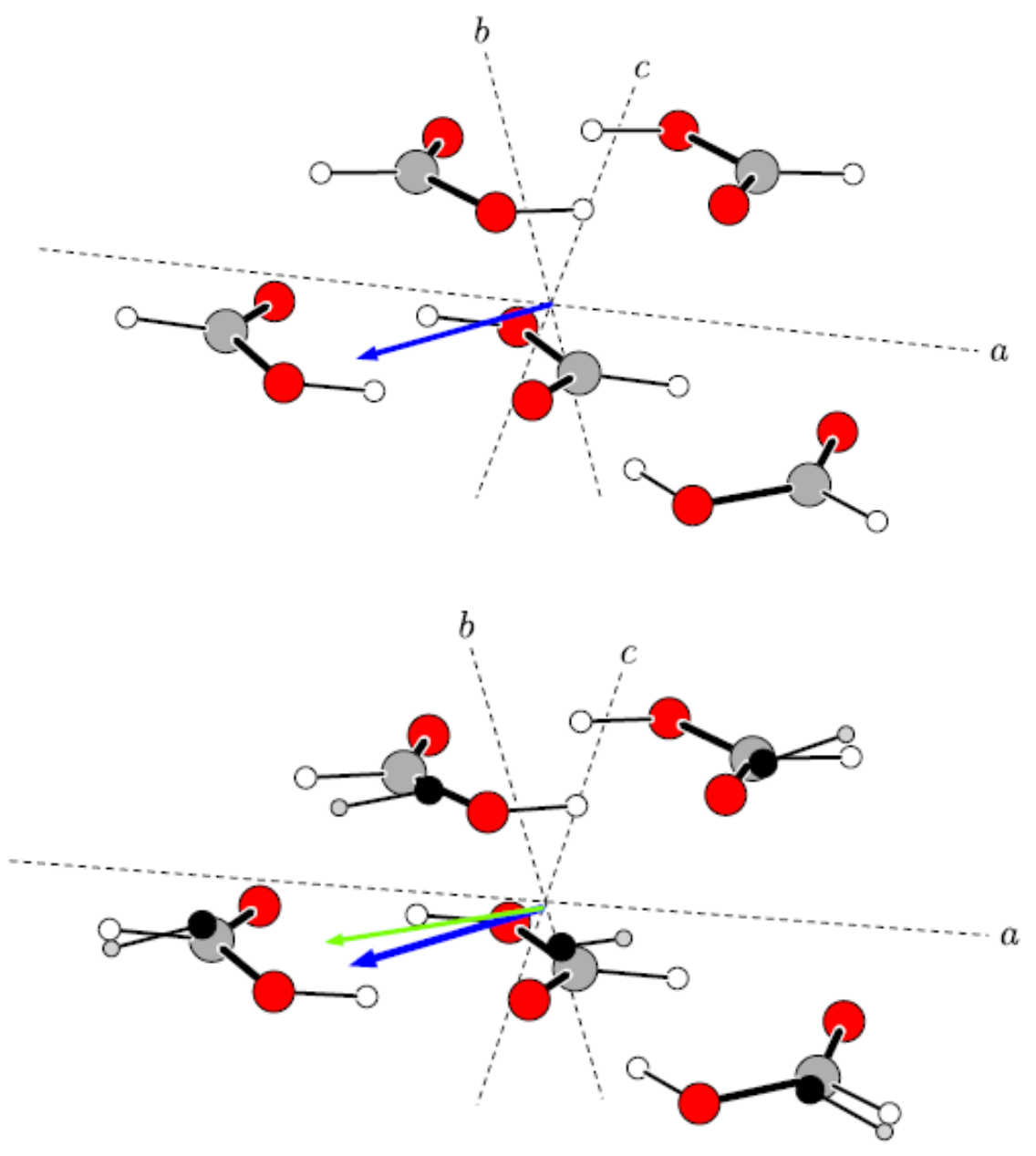

Figure 3.14. Structure of $(\mathrm{HCOOH})_{5}$. The top panel shows the proposed ab initio structure, calculated at an MP2/6-31++G(d,p) level of theory. The blue vector shows the direction of the calculated dipole moment. In the bottom panel, this structure is compared to the atom positions derived from the CP-FTMW measurements. The direction of the green arrow is determined from the observed relative intensities of the $a$, $b$ and $c$-type transitions; its length is fixed to that of the $a b$ initio dipole moment. 
except the carbonyl of the trimer subunit involved in a $\mathrm{C}-\mathrm{H} \ldots \mathrm{O}$ interaction (in Figure 3.14 , the carbonyl oxygen on the far right of the trimer unit) is tilted out of the plane, towards the dimer subunit. This tilt appears to be responsible for the chirality of the complex. The r.m.s. deviation between the experimental and calculated atom positions for the ten atom positions experimentally derived in this study was $0.403 \AA$; this error is significantly higher than for the smaller complexes, but the larger zero-point vibrational motions of this structure could explain these differences. A comparison of the experimentally fit and calculated Hamiltonian parameters is presented in Table 3.7. The relative dipole moment components, in particular, are not in extremely good agreement with calculation. However, as for the atom positions, the zero-point large-amplitude motions of the complex could make the calculation of the dipole moment more challenging.

Of all the structures calculated in this study, the proposed structure is not the lowest in energy, as another calculated structure is lower in energy by $32.6 \mathrm{~cm}^{-1}$ (without zero-point vibrational energy corrections). The rotational constants of this lowest-energy structure $(A=660.4 \mathrm{MHz}, B=374.1 \mathrm{MHz}, C=333.5 \mathrm{MHz})$ are in reasonable agreement with experiment. The dipole moment orientation is very different, however (predicting $\mu_{\mathrm{B}}$ to be the largest of the three components, while in fact experimentally it is the weakest), and the positions of the ten substituted atoms are in very poor agreement with experiment (r.m.s. deviation 1.278 $\AA$ ). This is shown in Figure 3.15. It is important to note that the direct structural information obtained through isotopic substitution is critical 
Table 3.7. Fit $A$-reduction Hamiltonian parameters of $(\mathrm{HCOOH})_{5}$, compared to values calculated by electronic structure theory.

\begin{tabular}{ccc}
\hline & & Ab Initio \\
Parameter & Experiment & MP2/6-31++G(d,p) \\
\hline$A(\mathrm{MHz})$ & $642.23341(15)$ & 638.10 \\
$B(\mathrm{MHz})$ & $375.92466(7)$ & 381.44 \\
$C(\mathrm{MHz})$ & $318.32987(7)$ & 330.32 \\
$\Delta_{\mathrm{J}}(\mathrm{kHz})$ & $0.03918(15)$ & $0.0244^{\mathrm{a}}$ \\
$\Delta_{\mathrm{JK}}(\mathrm{kHz})$ & $0.3196(6)$ & $0.1842^{\mathrm{a}}$ \\
$\Delta_{\mathrm{K}}(\mathrm{kHz})$ & $0.0023(17)$ & $-0.0635^{\mathrm{a}}$ \\
$\delta_{\mathrm{J}}(\mathrm{kHz})$ & $0.00257(7)$ & $0.00266^{\mathrm{a}}$ \\
$\delta_{\mathrm{K}}(\mathrm{kHz})$ & $0.1317(16)$ & $0.0885^{\mathrm{a}}$ \\
$\mu_{\mathrm{A}}(\mathrm{debye})$ & & 0.98 \\
$\mu_{\mathrm{B}}(\mathrm{debye})$ & & 0.57 \\
$\mu_{\mathrm{C}}(\mathrm{debye})$ & & 1.20 \\
$\left(\mu_{\mathrm{A}} / \mu_{\mathrm{B}}\right)^{2}$ & 5.3 & 2.96 \\
$\left(\mu_{\mathrm{A}} / \mu_{\mathrm{C}}\right)^{2}$ & 2.9 & 0.67 \\
$\mathrm{~N}_{\text {lines }}$ & 362 & \\
r.m.s. error $(\mathrm{kHz})$ & 8.3 &
\end{tabular}

${ }^{a}$ Centrifugal distortion constants were calculated at a B3LYP/6-31++G(d,p) level of theory (using the MP2/6-31++G(d,p) optimized structure). 

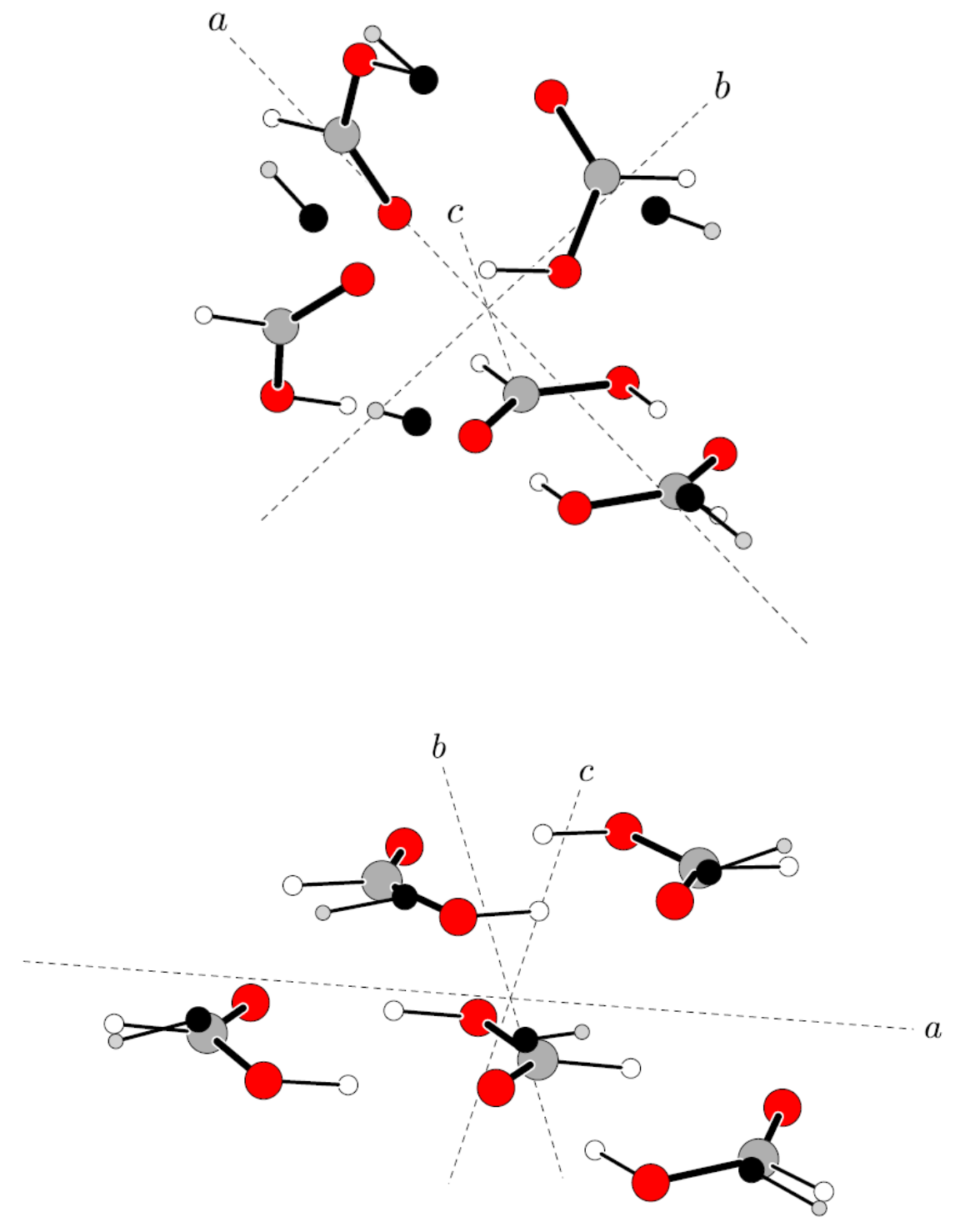

Figure 3.15. The two lowest-energy structures of $(\mathrm{HCOOH})_{5}$ calculated in this study, overlaid with the atom positions experimentally determined through isotopic substitution. The top structure is the lowest-energy structure calculated, while the bottom structure is higher in energy by $32 \mathrm{~cm}^{-1}$, and the two structures have very similar rotational constants. However, the agreement between the calculated and experimental atom positions, and the relative dipole moment components, show that the bottom structure is more likely to be the experimentally observed structure of $(\mathrm{HCOOH})_{5}$. 
to making a reasonably confident assignment in systems with complex potential energy surfaces like the formic acid pentamer. Several of the lowest-energy structures from the previous study by Roy and Thakkar were also calculated at an MP2/6-31++G(d,p) level of theory. The planar structures investigated, in particular, were far higher in energy than the structure assigned as the experimentally observed one (by at least $1000 \mathrm{~cm}^{-1}$ ). The previous study was performed using the B3LYP functional, which (as seen in the strawberry aldehyde section) tends to underestimate long-range dispersive interactions, which play a critical role in the structure of this complex. The nonplanar structures from Roy and Thakkar calculated in this study were found to be considerably lower in energy than the planar structures (though still higher in energy than the proposed experimentally observed structure).

If this complex is thought of as a cluster between the nonpolar formic acid dimer and the formic acid trimer structure assigned above, the strength of this non-hydrogenbonded interaction can be estimated from the binding energies of the clusters involved. The binding energies were calculated at the same level of theory as the rest of the calculations (MP2/6-31++G(d,p)) using the counterpoise method in Gaussian 09 to determine the basis set superposition errors. At this level of theory, the formic acid pentamer is calculated to have a total binding energy, as calculated by

$$
E_{\text {binding }}=E_{\text {pentamer }}-5 E_{\mathrm{FA}}-E_{\mathrm{BSSE}}
$$

of $150.0 \mathrm{~kJ} / \mathrm{mol}$. The complexation between the dimer and trimer does induce slight structural changes in the subunits, particularly in the trimer unit, for which there is a noticeable nonplanarity in the calculated structure. If we assume that the binding energy 
within these subunits is not significantly changed, we derive an interaction energy of $10.1 \mathrm{~kJ} / \mathrm{mol}\left(845 \mathrm{~cm}^{-1}\right)$ between the subunits (using calculated binding energies of 54.1 $\mathrm{kJ} / \mathrm{mol}$ for $(\mathrm{HCOOH})_{2}$ and $85.7 \mathrm{~kJ} / \mathrm{mol}$ for $\left.(\mathrm{HCOOH})_{3}\right)$.

\section{g) Conclusion}

In this section, the application of high-sensitivity CP-FTMW spectroscopy in the 7.0-18.5 GHz frequency range to the structure determination of complexes of formic acid has been described. The structures of three new complexes of formic acid, $(\mathrm{HCOOH})_{3}$, $(\mathrm{HCOOH})_{3}-\mathrm{H}_{2} \mathrm{O}$, and $(\mathrm{HCOOH})_{5}$, have been determined through the comparison of experimentally derived atom positions and dipole moment components to electronic structure theory. This technique offers an unbiased approach to molecular rotational spectroscopy that can elucidate structures even when electronic structure theory calculations are incomplete or incorrect due to the large number of interactions involved and the vast number of potential minima. The combination of deep signal average CPFTMW spectroscopy, coupled with double resonance and automated fitting techniques to assign spectra, can facilitate the rapid derivation of spectral assignments and molecular structures, which serve as a way to make library-free molecular identifications. The relatively new ability of this spectrometer to obtain isotopic information on large complexes, additionally, allows for the direct testing of electronic structure theory by $\mathrm{CP}$ FTMW spectroscopy, which can aid in the development and testing of new theoretical methods. 


\section{Chapter 3 References}

(1) Suenram, R. D.; Golubiatnikov, G. Y.; Leonov, I. I.; Hougen, J. T.; Ortigoso, J.; Kleiner, I.; Fraser, G. T. J. Mol. Spectrosc. 2001, 208, 188-193.

(2) Gunther, H. NMR Spectroscopy, 2nd ed.; John Wiley \& Sons: New York, 1995.

(3) Grubbs, G. S.; Dewberry, C. T.; Etchison, K. C.; Kerr, K. E.; Cooke, S. A. Rev. Sci. Instrum. 2007, 78, 096106-096103.

(4) Obenchain, D. A.; Elliott, A. A.; Steber, A. L.; Peebles, R. A.; Peebles, S. A.; Wurrey, C. J.; Guirgis, G. A. J. Mol. Spectrosc., 261, 35-40.

(5) Bird, R. G.; Neill, J. L.; Alstadt, V. J.; Young, J. W.; Pate, B. H.; Pratt, D. W. J. Phys. Chem. A 2011, in press.

(6) Steber, A. L.; Neill, J. L.; Zaleski, D. P.; Pate, B. H.; Lesarri, A.; Bird, R. G.; Vaquero-Vara, V.; Pratt, D. W. Faraday Discuss. 2011, in press.

(7) Emilsson, T.; Gutowsky, H. S.; de Oliveira, G.; Dykstra, C. E. J. Chem. Phys. 2000, 112, 1287-1294.

(8) Kasten, W.; Dreizler, H. Z. Naturforsch, A: Phys. Sci. 1984, 39, 1003.

(9) Muenter, J. S. J. Chem. Phys. 1968, 48, 4544-4547.

(10) Gordy, W., Cook, R.L. Microwave Molecular Spectra; John Wiley and Sons: New York, 1984.

(11) Kisiel, Z. PROSPE, Programs for ROtational SPEctroscopy, http://info.ifpan.edu.pl/ kisiel/prospe.htm.

(12) Nakajima, M.; Sumiyoshi, Y.; Endo, Y. Rev. Sci. Instrum. 2002, 73, 165-171. 
(13) Douglass, K. O.; Johns, J. E.; Nair, P. M.; Brown, G. G.; Rees, F. S.; Pate, B. H. J. Mol. Spectrosc. 2006, 239, 29-40.

(14) Suma, K.; Sumiyoshi, Y.; Endo, Y. Science 2005, 308, 1885-1886.

(15) This section contains material from a published manuscript: Shipman, S.T.; Neill, J.L.; Suenram, R.D.; Muckle, M.T.; Pate, B.H. J. Phys. Chem. Lett. 2011, 2, 443448.

(16) Shubert, V. A.; James, W. H.; Zwier, T. S. J. Phys. Chem. A 2009, 113, 80558066.

(17) Wiberg, K. B.; Laidig, K. E. J. Am. Chem. Soc. 1987, 109, 5935-5943.

(18) Pawar, D. M.; Khalil, A. A.; Hooks, D. R.; Collins, K.; Elliott, T.; Stafford, J.; Smith, L.; Noe, E. A. J. Am. Chem. Soc. 1998, 120, 2108-2112.

(19) Larson, J. R.; Epiotis, N. D.; Bernardi, F. J. Am. Chem. Soc. 1978, 100, 57135716.

(20) Tekin, A. R.; Karaman, H. Chem. Senses 1992, 17, 795-799.

(21) Zhao, Y.; Truhlar, D. G. Org. Lett. 2006, 8, 5753-5755.

(22) Zhao, Y.; Schultz, N. E.; Truhlar, D. G. J. Chem. Theor. Comput. 2006, 2, 364382.

(23) Zhao, Y.; Truhlar, D. G. J. Chem. Theor. Comput. 2006, 2, 1009-1018.

(24) Restrepo, A. A.; Bohn, R. K. J. Mol. Struct. 2007, 833, 189-196.

(25) Wodrich, M. D.; Corminboeuf, C.; Schleyer, P. Org. Lett. 2006, 8, 3631-3634.

(26) Holroyd, L. F.; van Mourik, T. Chem. Phys. Lett. 2007, 442, 42-46. 
(27) James, W. H.; Baquero, E. E.; Shubert, V. A.; Choi, S. H.; Gellman, S. H.; Zwier, T. S. J. Am. Chem. Soc. 2009, 131, 6574-6590.

(28) Barone, V.; Biczysko, M.; Pavone, M. Chem. Phys. 2008, 346, 247-256.

(29) Pietra, F. J. Phys. Org. Chem. 2007, 20, 1102-1107.

(30) Ruoff, R. S.; Klots, T. D.; Emilsson, T.; Gutowsky, H. S. J. Chem. Phys. 1990, $93,3142-3150$.

(31) Nesbitt, D. J. Chem. Rev. 1988, 88, 843-870.

(32) Legon, A. C. Annu. Rev. Phys. Chem. 1983, 34, 275-300.

(33) Birer, O.; Havenith, M. Annu. Rev. Phys. Chem. 2009, 60, 263-275.

(34) Balabin, R. M. J. Phys. Chem. A 2009, 113, 4910-4918.

(35) Madeja, F.; Havenith, M.; Nauta, K.; Miller, R. E.; Chocholousova, J.; Hobza, P. J. Chem. Phys. 2004, 120, 10554-10560.

(36) Brinkmann, N. R.; Tschumper, G. S.; Yan, G.; Schaefer, H. F. J. Phys. Chem. A 2003, 107, 10208-10216.

(37) Johnson, J. F.; Cole, R. H. J. Am. Chem. Soc. 1951, 73, 4536-4540.

(38) Bakó, I.; Schubert, G.; Megyes, T.; Pálinkás, G.; Swan, G. I.; Dore, J.; BellisentFunel, M.-C. Chem. Phys. 2004, 306, 241-251.

(39) Priem, D.; Ha, T.-K.; Bauder, A. J. Chem. Phys. 2000, 113, 169-175.

(40) Willemot, E.; Dangoisse, D.; Monnanteuil, N.; Bellet, J. J. Phys. Chem. Ref. Data 1980, $9,59-160$.

(41) Baskakov, O. I.; Winnewisser, B. P.; Medvedev, I. R.; De Lucia, F. C. J. Mol. Struct. 2006, 795, 42-48. 
(42) Hocking, W. H. Z. Naturforsch, A: Phys. Sci. 1976, 31a, 1113-1121.

(43) Goddard, J. D.; Yamaguchi, Y.; Schaefer III, H. F. J. Chem. Phys. 1992, 96, 1158-1166.

(44) Douglass, K. O. New Fourier Transform Microwave Techniques for Dynamic Rotational Spectroscopy, Ph.D. Thesis, University of Virginia, 2006.

(45) Ramón, J. M. H.; Ríos, M. A. Chem. Phys. 1999, 250, 155-169.

(46) Roy, A. K.; Thakkar, A. J. Chem. Phys. Lett. 2004, 386, 162-168.

(47) Kim, H.; Keller, R.; Gwinn, W. D. J. Chem. Phys. 1962, 37, 2748-2750.

(48) Kang, C.; Korter, T. M.; Pratt, D. W. J. Chem. Phys. 2005, 122, 174301-174308.

(49) Fleisher, A. J.; Morgan, P. J.; Pratt, D. W. J. Chem. Phys. 2009, 131, 211101211104.

(50) Allouche, A. J. Chem. Phys. 2005, 122, 234703-234711.

(51) Bicerano, J.; Schaefer, H. F.; Miller, W. H. J. Am. Chem. Soc. 1983, 105, 25502553.

(52) Plusquellic, D. F.; Lovas, F. J.; Pate, B. H.; Neill, J. L.; Muckle, M. T.; Remijan, A. J. J. Phys. Chem. A 2009, 113, 12911-12918.

(53) Roy, A. K.; Thakkar, A. J. Chem. Phys. Lett. 2004, 393, 347-354.

(54) Wang, W. Chem. Phys. Lett. 2005, 402, 54-56.

(55) Zhao, Y.; Truhlar, D. G. J. Phys. Chem. A 2005, 109, 6624-6627.

(56) Roy, A. K.; Thakkar, A. J. Chem. Phys. 2005, 312, 119-126. 


\section{Chapter 4}

\section{Spatial Distributions and Interstellar Reaction Processes ${ }^{1}$}

\section{Introduction}

Star-forming regions of our galaxy produce a rich organic and inorganic chemistry through a combination of surface and ice chemistry and gas-phase reaction processes. $^{2-4}$ Despite the low temperatures and pressures of interstellar clouds, it is possible to produce essentially the full range of organic species including alkanes, alkenes, alkynes, arenes, alcohols, ethers, aldehydes, ketone, esters, carboxylic acids, amines, and amides. ${ }^{5}$ Recent infrared observations ${ }^{6}$ suggest that this organic material includes the new forms of carbon, $\mathrm{C}_{60}$ and $\mathrm{C}_{70}$, that were first discovered 25 years in ago in laboratory experiments designed to create the novel species produced in carbon-rich stellar outflows. ${ }^{7,8}$ Because organic species are destroyed by high-energy radiation, the organic chemistry of space occurs in molecular clouds where small carbonaceous and silicate particles, called grains, scatter high frequency radiation and shield the molecular material. These "dark" regions of the sky, where the important astronomical processes of star and planet formation occur, can only be probed by long-wavelength light, and are therefore primarily studied at frequencies below $1 \mathrm{THz}$. There are several atmospheric windows in this low-frequency region that enable ground-based telescope facilities to be used for the study of the "cool universe" through radio astronomy.

An important feature of the light collected by radio astronomical observatories is that it contains the precise signatures of molecules arising from their molecular rotational spectra. This has led to a relatively recent advance in astronomy, as the first polyatomic 
molecules (ammonia, water, and formaldehyde) were observed by radio astronomy just 40 years ago. $^{9-11}$ Because of the unique spectral signatures provided by rotational spectroscopy, much of the chemical inventory of the interstellar medium has been detected using radio telescopes operating in the centimeter and millimeter wavelength region. Additionally, radio astronomical spectra contain information about the abundances, temperature, and kinematics of the molecular gas in star-forming regions, and molecules are often used as tracers to probe the structures of these astronomical objects.

The chemistry of the interstellar medium is known to consist of a combination of processes occurring in the gas phase and within dust grains. Gas phase chemistry, due to the low temperatures and densities of these regions, is dominated by reactions without activation barriers that can occur efficiently under these conditions. ${ }^{12}$ Ion-molecule reactions often satisfy this condition and are the most common class of gas-phase reactions proposed to be important in interstellar chemistry. Particularly for more complex molecules, however, the existence of efficient formation pathways has been cast into doubt by theoretical and experimental studies. Therefore, in recent years, increased study has been devoted to the processes by which complex molecules can form on the surfaces of interstellar ice grains. These surface reactions mainly involve radical-radical reactions that can take place at relatively low temperatures on the ices that build up on grains. ${ }^{13,14}$ Eventually the molecular material produced by the ice chemistry is evaporated into the gas phase during the warm-up phase associated with star formation, where they can then be detected at high spectral resolution by radio astronomical 
observations. A combination of observational, experimental, and theoretical work is being undertaken in a number of research groups to understand the relative importance of gas and grain chemistry in the development of molecular complexity in the interstellar medium.

Astronomers have routinely used single-dish telescopes, i.e. large filled-aperture instruments, to search for new molecules in interstellar clouds because such searches often require the high sensitivity offered by these instruments. Observations of molecular emission from radio telescope arrays, which correlate the signals from a collection of single-dish telescopes, have been largely limited to the strongest transitions of highly abundant molecules. Despite typically having lower sensitivity compared to single-dish observations, array observations are quite useful probes of interstellar chemistry because they provide spatial information about the molecular distribution that is not readily attainable with single-dish observations.

The next-generation radio astronomy observatories, such as the Expanded Very Large Array (eVLA) and the Atacama Large Millimeter/Submillimeter Array (ALMA), will provide an unprecedented view of the molecular composition of the Universe because they will incorporate both imaging capabilities and unparalleled sensitivity. A key feature of both of these observatories is broadband frequency coverage for imaging applications. For chemistry, this capability means that it will soon be possible to simultaneously obtain the spatial distributions of multiple chemical species. ALMA in particular will be able to generate chemical images with a higher spatial resolution than the Hubble Space Telescope, making it possible for chemical images to become well- 
known to the general public as a way to see the Universe. The focus of this chapter is to explore the potential of multispecies chemical maps to reveal mechanistic interstellar reaction chemistry through correlation of the spatial distributions of proposed reactant and product molecules.

The study presented here focuses on the synthesis of interstellar methyl formate, which has proven difficult to model by reaction kinetics networks. When only gas-phase production routes are included, the observed abundance of methyl formate is underpredicted. $^{15}$ Laboratory experiments that simulate interstellar chemistry have shown in recent years that reactions on ice surfaces can contribute to methyl formate production. ${ }^{16-18}$ It is difficult to disentangle the relative contributions of grain-surface and gas-phase chemistry to the formation of methyl formate using spectroscopic characterization alone, because such information provides only the relative abundances and temperatures of the molecules present in the cloud. It therefore remains unclear whether the reaction network implemented in current chemical models of interstellar clouds is complete, or if other chemical mechanisms that have not yet been included in these models might also contribute to methyl formate formation. Observational tests that differentiate between key grain-surface and gas-phase reactions could offer insight into the relative importance of these two pathways. Here multispecies chemical imaging is used to investigate whether the spatial distributions of methyl formate and related molecules are consistent with the chemistry currently included in the models, and to suggest other possible chemical reaction pathways for methyl formate synthesis under interstellar conditions. 
The observations that serve as a basis for this study are toward the Orion molecular cloud, which has attracted much astronomical interest due to its complex physical structure. Molecular observations play an important role in characterizing the different objects embedded in this complex environment by making it possible to visualize the outflow(s) of molecular material. ${ }^{19,20}$ From a chemical perspective, this region is characterized by a fascinatingly rich chemical inventory as well as extreme spatial differentiation between chemical species, identified through radio astronomy array measurements. $^{21,22}$ Two spatial regions have received special attention: the "Hot Core" and the "Compact Ridge." The Orion Hot Core is a warm region of molecular gas and dust, dominated by organic species containing nitrogen-based functional groups; the source of its heating is a matter of current debate. ${ }^{20}$ The neighboring Orion Compact Ridge, meanwhile, is dominated by oxygen-containing species, including methyl formate. The work presented herein focuses on molecular distributions in the Compact Ridge. The spatial distributions in this region of the Orion molecular cloud have been interpreted to indicate that molecular material has been evaporated from grain surfaces by the shocks of a molecular outflow from an astronomical object with high radio continuum emission called "I," proposed to be a highly luminous young star. ${ }^{19,23}$ The identity of this object is a topic of current interest. ${ }^{20,24,25}$ 


\section{Results}

\section{a) Chemical images from radio telescope arrays}

Obervations toward the Orion Kleinmann-Low (KL) region of star formation have been obtained using two radio telescope arrays, the eVLA and the Combined Array for Research in Millimeter Astronomy (CARMA). The eVLA observations were performed in December 2009 as part of the demonstration science for the recently upgraded array, operated by the National Radio Astronomy Observatory. ${ }^{26}$ This measurement was designed to test its new broadband spectral acquisition capabilities. The measurement was centered at a frequency of $25.2 \mathrm{GHz}$ and covered $3100 \mathrm{MHz}$ of spectral range. The spatial resolution was $5 . " 6 \times 2 . " 9$, corresponding to an average physical scale of 2500 AU (with 1 AU being approximately the mean earth-to-sun distance; our solar system has a radius of $30 \mathrm{AU})$. The total integration time on source was five hours.

The CARMA observations ${ }^{27}$ were conducted in December 2007 and July 2008. Two 7-hour tracks were taken in B configuration, and one 6-hour track was taken in D configuration. The typical synthesized beams are $6 . " 1 \times 5 . " 0$ for the D configuration and 1." $1 \times 0 . " 9$ for the B configuration. Given the projected baselines, structures larger than 30 arcseconds are resolved out. The correlator was configured during the commissioning phase of the CARMA array with six $31 \mathrm{MHz}$ wide windows for continuum and spectral lines (three windows in each sideband). The CARMA observations include chemical images from single rotational transitions of methyl formate, formic acid, and dimethyl ether. All chemical images presented represent the integrated spectral intensity of the monitored rotational transition; in some cases, all of the observed transitions in the 
passband for the same molecule were used to make the image. These images can be interpreted as a spatial representation of the molecular column density of the species. However, spatial variations in temperature can also cause the transition intensity to vary and could be partially responsible for the image morphology. A recent imaging study of methyl formate in Orion by Favre et al. ${ }^{28}$ probed several rotational transitions of methyl formate and could, therefore, separate column density and temperature effects. The methyl formate images presented here are consistent with those of Favre et al.

The spatial distributions of methyl formate and methanol from the eVLA observations are shown in Figure 4.1a. The region of highest methyl formate density is in the Orion Compact Ridge; it is slightly offset from the peak position of gas-phase methanol. The high spatial resolution mapping of methyl formate using CARMA is compared to the eVLA image in Figure 4.1b. This comparison shows that the morphology observed in the eVLA image is determined to a large extent by small-scale regions of methyl formate density, with the greatest density found at the position labeled MF1 (using the nomenclature of Favre $e t a l .^{28}$ ) The type of spectral information used to create this distribution image is shown in Figure 4.2, where the broadband rotational spectrum at the MF1 position is shown. Ten methanol transitions (the $K=2 \leftarrow 1 Q$ branch, $J=2-11$ ) are present in this spectrum, from which an approximate methanol temperature of $80 \mathrm{~K}$ is derived for this region through a least-squares fit to a Boltzmann distribution. It should be noted that the intensities of the transitions in this observation show significant deviations from local thermodynamic equilibrium behavior, but this 

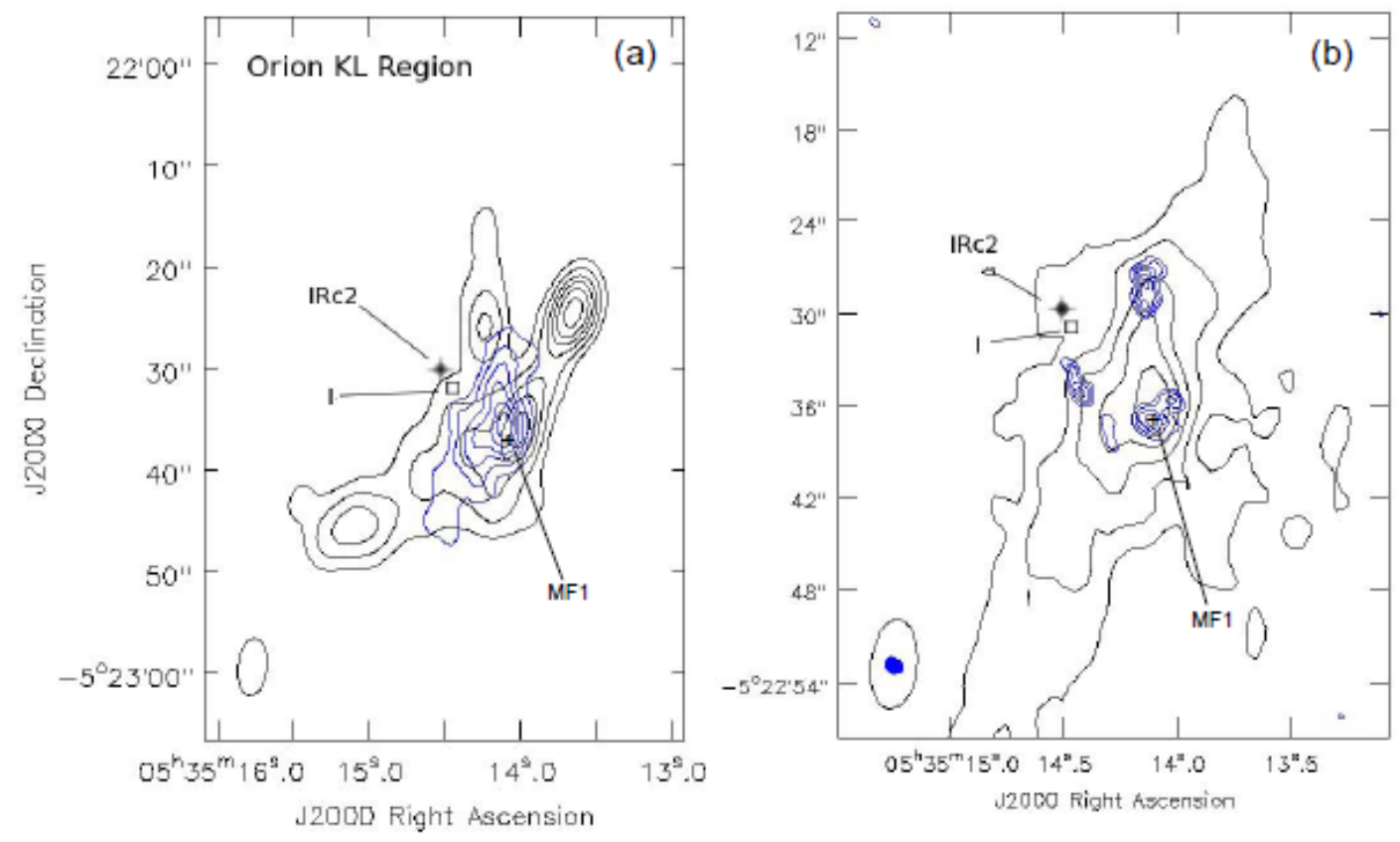

Figure 4.1. Panel (a): EVLA methyl formate (blue) (contour levels $=0.12,0.156,0.192$, $0.222,0.252$, and $0.276 \mathrm{Jy} / \mathrm{beam}$ ) and methanol (black) (contour levels $=11,22,35.75$, 49.5, 66, 88, and $110 \mathrm{Jy} / \mathrm{beam}$ ). Panel (b): CARMA methyl formate (blue) (contour levels $=0.2,0.3,0.4,0.5$, and $0.6 \mathrm{Jy} / \mathrm{beam}$ ) and EVLA methyl formate (black) (contour levels $=0.12,0.156,0.192,0.222,0.252$, and $0.276 \mathrm{Jy} / \mathrm{beam})$. The locations of the star IRc2, the "I" radio continuum source, and the MF1 location of methyl formate emission following the nomenclature of Favre et ll $^{28}$ are labeled. The synthesized beam widths are indicated in the lower left corner of each panel. 


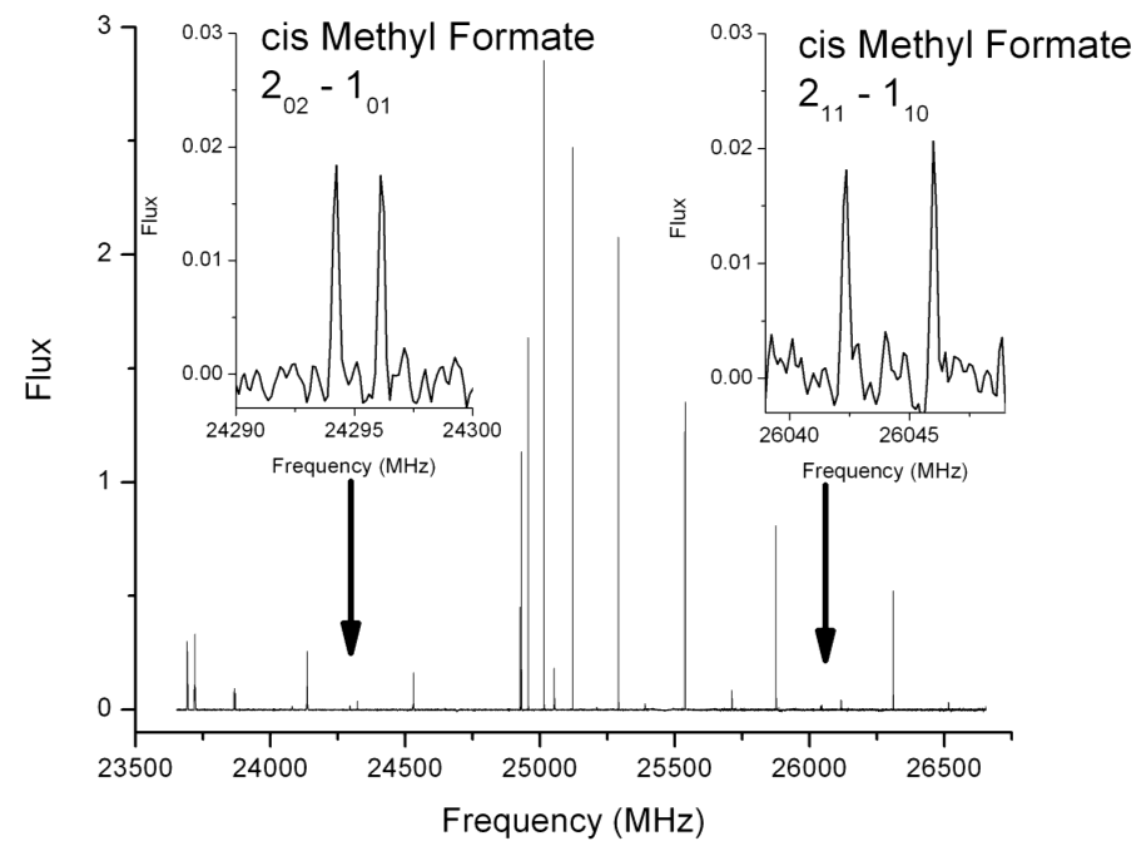

Figure 4.2. The eVLA spectrum of the Orion molecular cloud at the position of highest methyl formate density. The strongest transitions originate from the $K=2 \leftarrow 1 Q$-branch of methanol, while the two transitions of cis-methyl formate in this frequency range are shown as insets. 
rough estimation should still be sufficient for the analysis in this chapter. This temperature is similar to that reported by Favre et al. ${ }^{28}$ who derived an average methyl formate rotational temperature of $100 \mathrm{~K}$ in the MF1 region, using measurements with two different spatial resolutions. Using this information, the relative abundance ratio of methanol to methyl formate in the spectrum in Figure 4.2 is estimated to be $\sim 15$, which is roughly consistent with the molecular abundances in the Orion Compact Ridge by Sutton et al. ${ }^{29}$ which are listed in Table 4.1.

Three CARMA images are shown in Figure 4.3. The first image, Figure 4.3a, compares the low spatial resolution images of formic acid and methyl formate. A simple examination of these two distributions shows that methyl formate production near MF1 comes at the expense of formic acid, which shows an apparent decrease in its density with a morphology that complements that of methyl formate. The higher spatial resolution image of methyl formate is compared to that of formic acid in Figure 4.3b. The region of highest methyl formate production is found in the area of formic acid depletion. The formic acid distribution shows no small-scale spatial regions of enhanced production in the higher spatial resolution images. The low-resolution CARMA images of dimethyl ether and methyl formate are compared in Figure 4.3c. There is a striking correlation between the distributions of methyl formate and dimethyl ether, suggesting that they are formed through chemically related reaction processes (that differ from formic acid production processes). The high-resolution CARMA image of dimethyl ether (not shown) indicates that the position MF1 is also the location of peak dimethyl ether formation. 
Table 4.1. Abundances of molecules potentially related to methyl formate production in the Orion Compact Ridge.

\begin{tabular}{cc}
\hline Species & $10^{9} \times$ Fractional Abundance ${ }^{a, b}$ \\
\hline $\mathrm{HCOOCH}_{3}$ & 30 \\
$\mathrm{HCOOH}$ & 1.4 \\
$\mathrm{CH}_{3} \mathrm{OCH}$ & 19 \\
$\mathrm{H}_{2} \mathrm{CO}$ & 40 \\
$\mathrm{CH}_{3} \mathrm{OH}$ & 400
\end{tabular}

\footnotetext{
${ }^{a}$ Relative to $\mathrm{H}_{2}$, assuming that the molecule and $\mathrm{H}_{2}$ are coextensive.

${ }^{b}$ Values from Sutton et al. ${ }^{29}$
} 


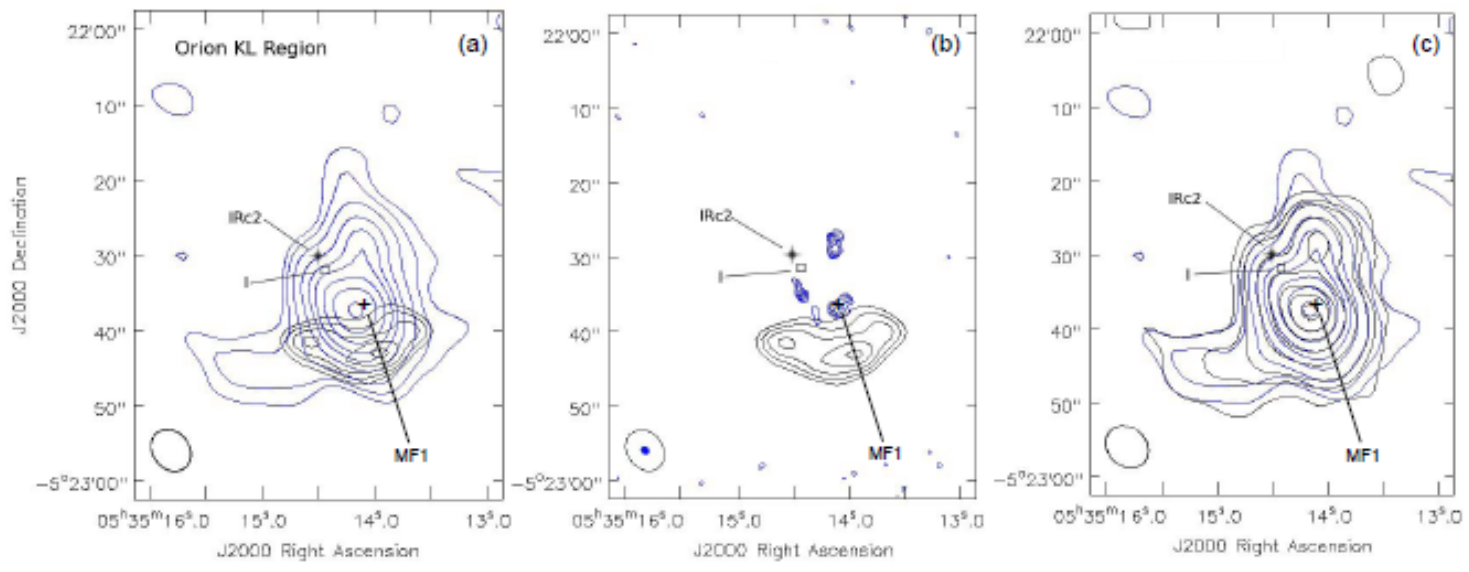

Figure 4.3. Panel (a): CARMA methyl formate (blue) (contour levels $=0.08,0.16,0.32$, $0.64,1.04,1.44,2.08,2.88$, and $3.84 \mathrm{Jy} /$ beam) and formic acid (black) (contour levels $=$ $0.138,0.162,0.186,0.21$, and $0.228 \mathrm{Jy} / \mathrm{beam})$. Panel (b): CARMA methyl formate (blue) (contour levels $=0.2,0.3,0.4,0.5$, and $0.6 \mathrm{Jy} / \mathrm{beam}$ ) and formic acid (black) (contour levels $=0.138,0.162,0.186,0.21$, and $0.228 \mathrm{Jy} / \mathrm{beam})$. Panel (c): CARMA methyl formate (blue) (contour levels $=0.08,0.16,0.32,0.64,1.04,1.44,2.08,2.88$, and $3.84 \mathrm{Jy} / \mathrm{beam}$ ) and dimethyl ether (black) (contour levels $=0.05,0.15,0.25,0.65,1.2$, $1.75,2.15$, and $2.55 \mathrm{Jy} / \mathrm{beam})$. The synthesized beam widths are indicated in the lower left corner of each panel. 


\section{b) Interpretation of chemical images based on chemical reaction processes}

In the remainder of this chapter, the proposal that the spatial distributions of multiple chemical species can be used to infer chemical mechanisms that relate these molecules is tested. A fully quantitative treatment requires that all reactions linking the various chemical species be included in the chemical reaction networks, and that spatial effects be added to the physical model through incorporation of hydrodynamics. The current state-of-the-art approaches for both of these key factors remain incomplete, limiting the interpretation of these new methyl formate observations to semi-quantitative results. However, it should be stressed that this work is one of the first studies to examine chemical mechanisms through spatial distributions, and as such serves as the foundation for development of a full quantitative treatment of this problem.

The addition of spatial distributions to interstellar reaction networks is a field of study in its infancy. The first report of the predicted spatial distribution of molecules for hot core chemistry indicates that the gas-phase distributions of methyl formate, formic acid, dimethyl ether, and methanol are highly correlated with a peak abundance centered on the hot core position. ${ }^{30}$ Recent laboratory experiments of the temperature programmed desorption of organic ices shows that these four species have similar desorption temperatures. ${ }^{17}$ Therefore, desorption from the shocks of a molecular outflow might also be expected to produce similar spatial distributions of these species. In fact, the low spatial resolution images from CARMA show that the formic acid, methyl formate, and dimethyl ether distributions have a similar large-scale distribution from eastto-west that is most clearly seen in the formic acid image. 
As discussed above, the Orion Compact Ridge shows several deviations from this simple model. Recent gas-grain chemistry models predict that at the temperature of $\sim 80$ $100 \mathrm{~K}$ estimated for this region, formic acid is found in an abundance equal to or greater than that of methyl formate. ${ }^{13,14}$ However, no local enhancement of formic acid column density is observed near the MF1 position. As a result, gas-phase chemical reactions that can "post-process" the organic material liberated from the grains in the Orion Compact Ridge region are considered. Presumably all of the material formed on grain surfaces would be deposited through the same physical mechanism (i.e. heating, shock, etc.), and therefore the chemical products of this process would display similar spatial distributions. However, subsequent gas-phase reaction chemistry could reshape the molecular distributions, and, therefore, is more likely to be revealed by studying the correlations of different molecular spatial distributions. The distributions of low-abundance species (i.e. the limiting reagents) will be most strongly affected by chemical processing. The hypothesis that we consider here is that the spatial distributions of methyl formate and formic acid suggest that there is a gas-phase reaction process that consumes formic acid to produce methyl formate. This idea is suggested by the apparent reduction in formic acid density at the position of peak methyl formate production (MF1). It should also be noted that the same anticorrelation of column densities is seen even more strongly in a different region of interstellar chemistry (IRAS 16293-2422), where the methyl formate density peaks in a "valley" of formic acid density. ${ }^{31}$ The correlation of the spatial distributions of methyl formate and formic acid in IRAS 16293-2422 can be seen in 
Figure 4.4. The timescales for gas-phase processing of the material in this low-mass hot core compared to the high-mass Orion star forming region make direct comparison between the two sources difficult without a full understanding of the relative contributions of gas and grain chemical processes. However, this anticorrelation between methyl formate and formic acid emission does provide a hint at possible reaction pathways that involve the formation of methyl formate from formic acid. Finally, the spatial distributions of methyl formate and dimethyl ether have a strong correlation, with peak production occurring near the same position. Therefore, any proposed reactions should be consistent with the close correlation between these structurally similar molecules.

Based on the proposed image interpretation, two possible methyl formate production reactions have been examined using computational chemistry. To produce significant amounts of methyl formate by gas-phase reactions, at least one of the reactants must be a high-abundance interstellar molecule. As shown in Table 4.1, formaldehyde and methanol are found in high abundance in the Compact Ridge region. Ion-molecule chemistry involving these molecules could be facilitated by the protonation of these highabundance species through proton transfer with a highly-abundant interstellar ion such as $\mathrm{H}_{3}{ }^{+}$or $\mathrm{HCO}^{+}$that has a low proton affinity. One of the early proposals for interstellar methyl formate production was the reaction of protonated methanol with formaldehyde ${ }^{32,33}$ however, a computational chemistry study revealed that the barrier of this reaction is too high $(128 \mathrm{~kJ} / \mathrm{mol})$ for this reaction to contribute significantly to methyl 


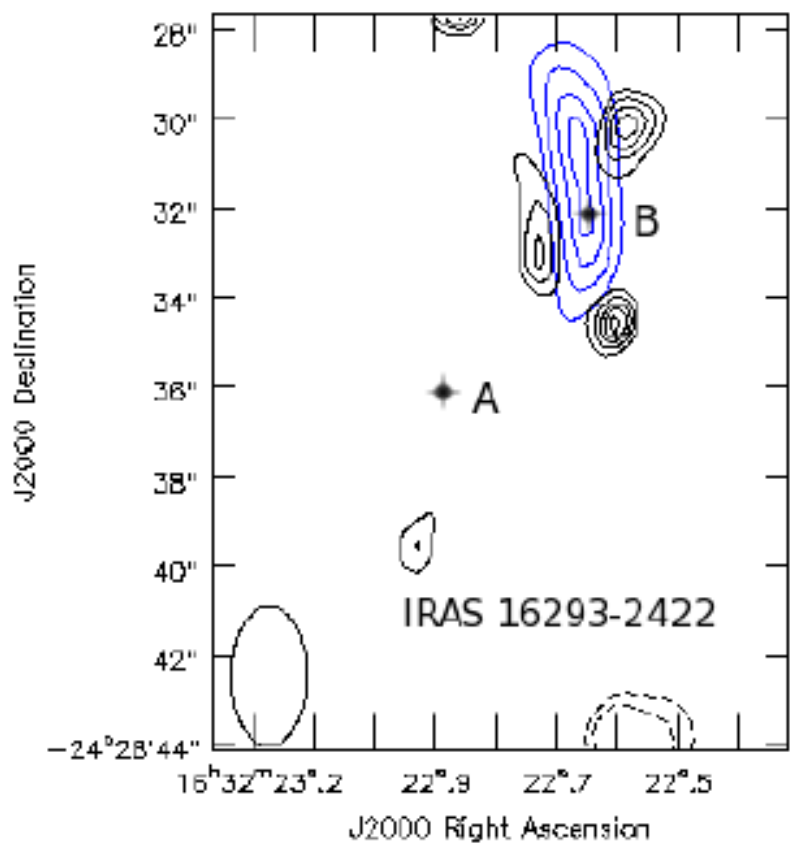

Figure 4.4. VLA spatial correlations of methyl formate (black) (contour levels $=0.0115$, $0.0135,0.015$, and $0.01585 \mathrm{Jy} / \mathrm{beam}$ ) and formic acid (black) (contour levels $=-0.0154$, $0.0175,0.014,0.0154,0.01645,0.01715$, and 0.01785) in IRAS 16293-2422. These data come from the previous study by Remijan and Hollis. ${ }^{31}$ The synthesized beam width is indicated in the lower left corner. The labels " $A$ " and " $\mathrm{B}$ " indicate the positions of the two major cores in this region. 
formate synthesis under interstellar conditions. ${ }^{15}$ The ion-molecule reactions considered here involve the reaction of methanol and formic acid, where one of the reactants is protonated. These reactions were not considered in the previous computational study of the gas-phase production of methyl formate. ${ }^{15}$

\section{c) Methyl formate production via acid-catalyzed Fischer esterification}

The reaction between neutral methanol and protonated formic acid is well-known in organic chemistry as acid-catalyzed Fischer esterification:

$$
\mathrm{CH}_{3} \mathrm{OH}+\left[\mathrm{HC}(\mathrm{OH})_{2}\right]^{+} \rightarrow\left[\mathrm{HC}(\mathrm{OH}) \mathrm{OCH}_{3}\right]^{+}+\mathrm{H}_{2} \mathrm{O}
$$

The reaction profile obtained from $a b$ initio calculations is shown in Figure 4.5. In this study, we consider two approach geometries for the reactants. These different pathways have transition states that lead to different conformers of protonated methyl formate. The calculated reaction barriers are $17 \mathrm{~kJ} / \mathrm{mol}$ and $21 \mathrm{~kJ} / \mathrm{mol}$ for paths that produce cis- or trans-protonated methyl formate as products, respectively. (For comparison, the reaction barrier in the absence of acid catalysis is about $160 \mathrm{~kJ} / \mathrm{mol}$ at the same level of theory.)

There are several aspects of this reaction which deserve additional comment: (1) At the temperatures estimated for the Orion Compact Ridge ( 100 K), the thermal energy $\mathrm{kT}$ is only about $1 \mathrm{~kJ} / \mathrm{mol}$, suggesting that the direct reaction by this mechanism is inefficient. It does have a stable entrance channel complex, however, and the vibrational mode with an imaginary frequency at the transition state is essentially a single hydrogen atom motion (reduced mass $1.09 \mathrm{u}$ ), meaning that if the complex were collisionally or 


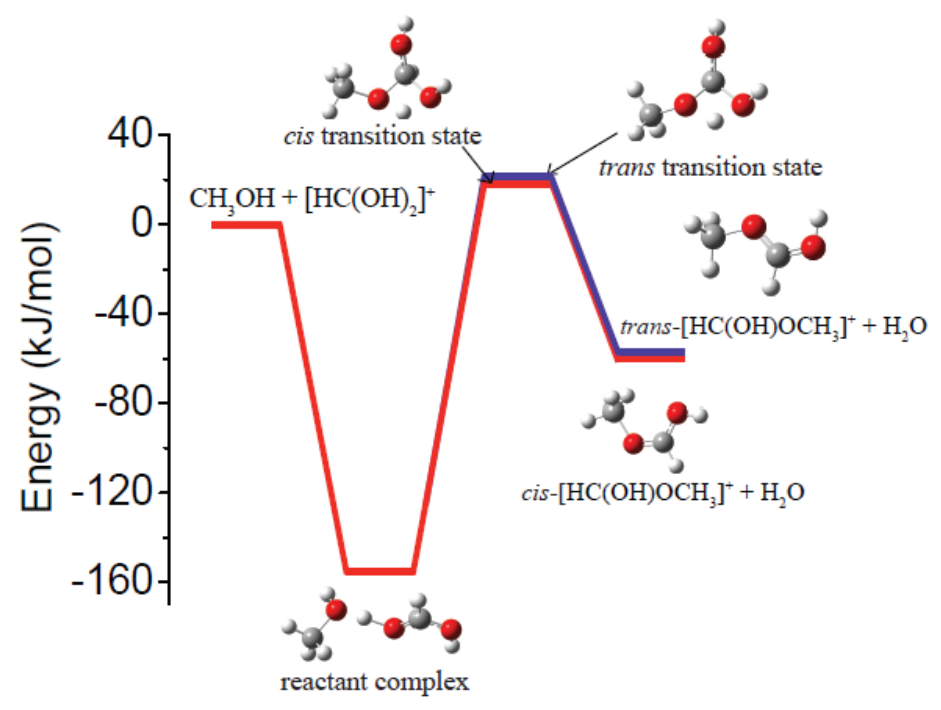

Figure 4.5. Energetic profile of the acid-catalyzed Fischer esterification reaction of neutral methanol and protonated formic acid, calculated at a M06-2X/6-31+G(d,p) level of theory. The energies have been corrected to account for zero-point vibrational energy. The red line corresponds to the formation of cis-protonated methyl formate, while the blue line corresponds to the formation of trans-protonated methyl formate. 
radiatively stabilized, ${ }^{34-36}$ protonated methyl formate could be formed via tunneling. ${ }^{37,38}$ (2) This reaction also requires a large abundance of protonated formic acid, but $\mathrm{H}_{3}{ }^{+}$, which plays a central role in interstellar ion-molecule chemistry, ${ }^{12,39,40}$ cannot protonate formic acid directly, as this reaction instead results in molecular dissociation. ${ }^{41,42}$ Instead, protonation of formic acid must occur by another ion, such as $\mathrm{HCO}^{+}$or $\mathrm{H}_{3} \mathrm{O}^{+}$, either of which has been shown in the laboratory to efficiently form this species. ${ }^{41-43}$ (3) Proton transfer between the reactants is exothermic and may be more facile; two laboratory studies have found that this proton transfer pathway is the dominant reaction channel. $^{44,45}$ (4) It has also been suggested that the gas-phase esterification reaction does not proceed through the traditional Fischer mechanism, with a tetrahedral intermediate. A recent study ${ }^{46}$ suggests that a closely related reaction, that of protonated acetic acid and methanol, may instead proceed through a proton-shuttling mechanism, and might not have a reaction barrier. Due to the complexity of this pathway, the authors were unable to determine the complete reaction path; additional calculations are needed to determine whether acid-catalyzed Fischer esterification is a viable way to produce interstellar methyl formate via this mechanism.

\section{d) Methyl formate production via methyl cation transfer}

The second possibility for the gas-phase formation of methyl formate is the reaction between protonated methanol and neutral formic acid, a reaction previously considered by Ehrenfreund and Charnley as a possible route for interstellar methyl formate production, ${ }^{4}$ 


$$
\mathrm{HCOOH}+\left[\mathrm{CH}_{3} \mathrm{OH}_{2}\right]^{+} \rightarrow\left[\mathrm{HC}(\mathrm{OH}) \mathrm{OCH}_{3}\right]^{+}+\mathrm{H}_{2} \mathrm{O}
$$

The reaction profiles for two different approach geometries are shown in Figure 4.6. In this reaction, a methyl cation is transferred to neutral formic acid to create protonated methyl formate and water. Gas-phase alkyl cation transfer reactions with protonated alcohols have been studied extensively in the laboratory. ${ }^{47-49}$ For this reaction profile, only the more stable conformation of formic acid as the reactant has been considered, since the less stable conformer has not been detected in the interstellar medium. Determination of the energy of the products assumes the conformation of protonated methyl formate that is most likely to be produced based on the transition state geometry. This is the reason that the energies of the products in Figures 4.5 and 4.6 differ; the conformation of protonated methyl formate produced by the two reactions is different in the orientation of the hydroxyl proton.

In contrast to the Fischer esterification reaction, calculations predict a barrierless process for the formation of protonated methyl formate in the trans conformation of the ester, while the reaction that leads to cis-protonated methyl formate has a barrier of about $10 \mathrm{~kJ} / \mathrm{mol}$. The reaction between formic acid and protonated methanol has been studied in the laboratory using the flowing afterglow technique at $300 \mathrm{~K}$, but only products involving proton transfer between the reactants were observed. ${ }^{42}$ This is an endothermic proces $^{50}$ that is likely of little significant at typical interstellar temperatures. Unlike formic acid, methanol can be readily protonated by $\mathrm{H}_{3}{ }^{+}$; two independent experimental studies have found significant product branching ratios ( 25-30\% in the low-pressure 


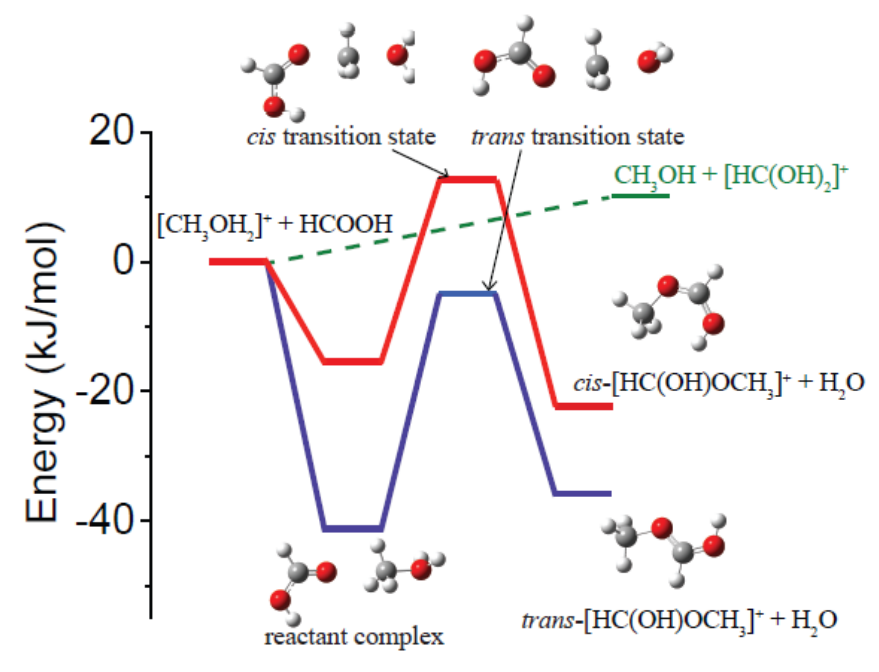

Figure 4.6. Energetic profile of the methyl cation transfer reaction of protonated methanol and neutral formic acid, calculated at a M06-2X/6-31+G(d,p) level of theory. The energies have been corrected to account for zero-point vibrational energy. The red line corresponds to the formation of cis-protonated methyl formate, while the blue line corresponds to the formation of trans-protonated methyl formate. The green line shows the energetics of the proton transfer reaction. 
limit) for the creation of protonated methanol. ${ }^{51,52}$ For this reason, protonated methanol is likely to be an abundant interstellar species. However, owing to the lack of rotational transition frequencies, this ion has not yet been detected in space.

It is also worth emphasizing that this reaction profile is energetically similar to that between protonated methanol and neutral methanol to produce protonated dimethyl ether; it too occurs without a barrier, ${ }^{53}$ and is considered an important contributor to interstellar dimethyl ether formation. ${ }^{54}$ If the methyl cation transfer reaction efficiently produces methyl formate in the MF1 region, then one would expect dimethyl ether would also be produced in the same spatial region, since neutral methanol is also present in this region. The close correlation between the methyl formate and dimethyl ether molecular distributions shown in panel (c) of Figure 4.3 is consistent with a reaction chemistry linked by protonated methanol as a reagent.

\section{e) Dissociation of protonated methyl formate}

Both reactions, but particularly the barrierless methyl cation transfer reaction, would be expected to produce significant amounts of protonated methyl formate in the less stable trans conformation of the ester, but only the neutral form of methyl formate in its lower energy cis conformer has been detected previously by radio astronomical observations. The neutral form can be produced from protonated methyl formate by dissociative electron recombination or proton transfer to a high-abundance species with higher proton affinity. 
Dissociative recombination reactions of several organic ions of interstellar interest have been studied in recent years using the heavy ion storage ring CRYRING. ${ }^{55-59}$ These studies find that molecular fragmentation is a major product channel owing to the large exothermicity for this type of neutralization reaction. However, chemical kinetics models recently calculated that the gas-phase synthesis of dimethyl ether through its protonated counterpart could produce the observed abundance of neutral dimethyl ether in the interstellar medium, even assuming a low branching fraction for the production of neutral dimethyl ether. ${ }^{13}$ Despite the apparent inefficiency of the dissociative recombination process, ion-molecule synthetic routes still have great significance in interstellar chemistry.

Although a study of methyl formate dissociative recombination has not been published, a recent study of structurally similar formic acid has been reported. ${ }^{59}$ This study found that $13 \%$ of reactions did not result in any heavy atom bond breaking. The exact composition of the remaining $87 \%$ could not be quantitatively determined, but energetically, the two most favorable processes are molecular dissociations leading to $\mathrm{CO}_{2}+\mathrm{H}_{2}$ and $\mathrm{CO}+\mathrm{H}_{2} \mathrm{O}$, with barriers of $\sim 290 \mathrm{~kJ} / \mathrm{mol} ;{ }^{60}$ bond cleavage, alternatively, to form $\mathrm{HCO}+\mathrm{OH}$, requires $450 \mathrm{~kJ} / \mathrm{mol}$ of energy. ${ }^{61}$ Methyl formate can dissociate with qualitatively similar barriers. $^{62,63}$ Because of its high proton affinity, additionally, the methyl formate dissociative recombination reaction is less exothermic than that of formic acid, ${ }^{64}$ so it is possible that the formation of neutral methyl formate could have a higher branching ratio than the corresponding formic acid reaction. 
There is also the possibility that the conformational specificity of the proposed reactions could be degraded in the conversion to neutral methyl formate. Nevertheless, if these ion-molecule reactions make a meaningful contribution to the total production of gas-phase interstellar methyl formate, then it is likely that the less stable trans conformer of neutral methyl formate would be produced in a high enough abundance for its radio astronomical detection. The conformational potential energy surface of methyl formate is presented in Figure 4.7. The calculations presented here are consistent with those of Senent et al. ${ }^{65}$ The trans conformer is less stable than cis-methyl formate by about 25 $\mathrm{kJ} / \mathrm{mol}$, with the difference in conformer stability arising from electrostatic effects rather than a steric interaction. ${ }^{66,67}$ The conformational potential energy surface of methyl formate is very similar to that of formic acid. ${ }^{60,68,69}$ Under thermodynamic equilibrium at $100 \mathrm{~K}$, the abundance of the trans conformer is about $10^{-14}$ of that of the cis conformer, which would make the trans conformer undetectable by radio astronomy. Because the barrier for the interconversion from trans to cis is calculated to be $\sim 35 \mathrm{~kJ} / \mathrm{mol}$, it is probable that any neutral trans-methyl formate produced will remain in this conformation under interstellar conditions. Interstellar detections of trans-methyl formate could provide strong, albeit circumstantial, evidence for gas-phase ion-molecule reactions that contribute significantly to interstellar methyl formate production.

In Chapter 5, motivated by the work presented in this chapter, the first laboratory assignment of the rotational spectrum of trans-methyl formate using CP-FTMW spectrometers, cavity FTMW spectrometers, and microwave-microwave double 


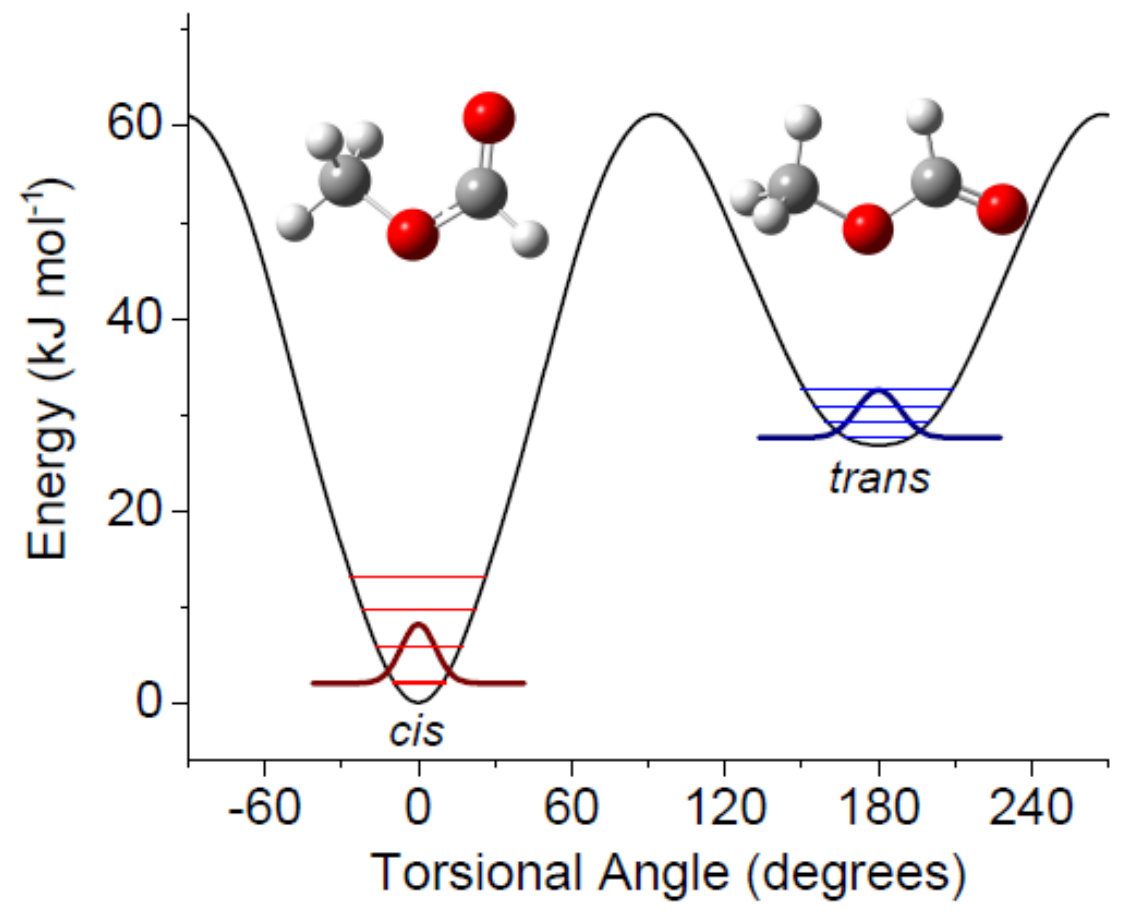

Figure 4.7. Conformational potential energy surface of methyl formate, calculated at an MP2/6-31++G(d,p) level of theory. 
resonance spectroscopic techniques will be presented. Because of the structural similarity of ethyl formate to methyl formate, and the fact that ethyl formate in its lowestenergy isomeric form is a newly detected interstellar molecule, ${ }^{70}$ the analogous conformer of ethyl formate, with a trans ester and gauche ethyl orientation, is also presented using the same techniques. For both experiments, a pulsed discharge nozzle source was used to enhance the thermodynamic equilibrium populations of these weak conformers by approximately a factor of $30 .^{71}$ Additionally, the first interstellar detection of trans-methyl formate in the Sagittarius B2(N) molecular cloud will be presented. This detection is in a different source for interstellar chemistry than the interferometric observations presented above; however, this detection demonstrates that the less stable conformation of methyl formate can be produced by reactions that are feasible under interstellar conditions. Further details of this detection and its implications for the interstellar chemistry of methyl formate will be presented in the next chapter.

\section{Discussion}

The fundamental premise of this work is that the interstellar spatial distributions of chemically related species are influenced by the reaction chemistry and, therefore, convey information about the chemical production routes; however, astrophysical effects could also lead to the observed correlations and anticorrelations. Modeling studies have shown that observed molecular abundances are highly dependent on the temperature and warm-up timescale. ${ }^{13,14}$ Examination of the temperature-dependent results from Laas $e t$ $a l{ }^{72}$ shows that methyl formate is formed efficiently on grain surfaces in the range of 30- 
$70 \mathrm{~K}$. At temperatures greater than $50 \mathrm{~K}$, the methyl formate gas-phase abundance begins to increase as a result of its evaporation from the ice. The grain-surface and gasphase abundances of formic acid are markedly different from those of methyl formate in the same temperature range, remaining essentially constant in both cases. It is only at higher temperatures ( $T>90 \mathrm{~K}$, i.e. where the water ice matrix begins to evaporate) that the gas-phase formic acid abundance begins to increase. It is possible, then, for methyl formate to be liberated from ice mantles and formic acid to still be trapped in the water ice matrix in regions where the local temperature is in the range of $70-90 \mathrm{~K}$. The gasphase methyl formate abundance would peak in these slightly colder regions, whereas the formic acid abundance would peak in warmer regions.

In addition to the more general case of temperature differentiation, a mechanism allowing for the sudden release of ice mantles (i.e. shocks) would lock the gas-phase abundances into the relative ratios dictated by the grain surface chemistry at the temperature of the event. The spatial distribution of formic acid in the Orion Compact Ridge has been interpreted to represent a shock boundary, as a number of water masers can be found near the region of highest formic acid abundance. ${ }^{73}$ The observed gasphase organic material would have been liberated from grains in the pre-shock region south of MF1. In Figure 4.3 it can be seen that in this region, indeed, methyl formate, dimethyl ether, and formic acid display similar spatial distributions. The new gas-phase reactions proposed in this paper are based on molecular abundances in the MF1 region, where formic acid is present at a far lower abundance than methyl formate or dimethyl ether. This region could be one that is shielded from the outflow, or one where post- 
shock chemistry has occurred. Regardless of the delivery mechanism for the gas-phase material, it is quite likely that this material will be additionally processed in regions where the timescales are sufficiently long for gas-phase reactions to occur. Further molecular spatial mapping of other interstellar environments with high image fidelity in the coming years will help to clarify the role of chemical and astrophysical effects in shaping molecular distributions.

There are a number of further studies that are suggested by the work presented in this chapter. Progress has already been made on a few of these projects: as mentioned above, the laboratory microwave spectra of trans-methyl formate and the analogous trans-gauche conformer of ethyl formate have been detected, and trans-methyl formate has been detected in the Sagittarius B2(N) molecular cloud. Observations of transmethyl formate in the Orion Compact Ridge will be important to determine if it is being produced in the MF1 region. The protonated forms of methanol, dimethyl ether, and methyl formate are new targets for laboratory rotational spectroscopy, as they might be present at sufficiently high column densities to enable detection if these proposed reactions are indeed important in these environments. A modeling study including the

two new gas-phase reaction processes presented here has been published, ${ }^{72}$ suggesting that the trans conformer of methyl formate could be produced at a detectable abundance in warm regions by the methyl cation transfer reaction. 


\section{Conclusion}

Multispecies chemical images of chemically rich regions of the interstellar medium have been used to examine the production of methyl formate in the Compact Ridge region of the Orion molecular cloud. The correlation of the column density profiles of low abundance species was used to suggest reaction partners in a chemical reaction. The chemical images in Orion and another interstellar region, IRAS 16293-2422, suggest the gas-phase conversion of formic acid to methyl formate. Two possible ion-molecule reactions between formic acid and methanol investigated by computational chemistry are suggested to be viable interstellar reaction routes. The conformer specific reaction paths suggest that these reactions could lead to a relatively high abundance of the less stable trans conformer of methyl formate under the kinetically-controlled conditions expected to hold in interstellar reaction environments.

This work suggests that the coming availability of high spatial resolution chemical images from next-generation radio astronomical observatories will provide a new way to explore mechanistic interstellar chemistry. The unusual chemical environments in the interstellar medium can produce novel chemical reaction processes that complement the synthesis techniques common under terrestrial conditions with the potential to generate new types of chemical species and materials. This chemistry may also play a key role in providing the basic chemical materials to newly forming planetary systems that can, under favorable conditions, possibly develop life. A better understanding of the chemical reaction processes at work in different interstellar environments can also be used to advance our understanding of formation of important 
astronomical objects, including stars and planets. The development of analysis methods to interpret multispecies chemical images from radio astronomy has the potential to make chemistry a fundamental tool for exploring the structure and evolution of the Universe. 


\section{Chapter 4 References}

(1) This chapter contains material from a published manuscript: Neill, J.L.; Steber, A.L.; Muckle, M.T.; Zaleski, D.P.; Lattanzi, V.; Spezzano, S.; McCarthy, M.C.; Remijan, A.J.; Friedel, D.N.; Widicus Weaver, S.L.; Pate, B.H. J. Phys. Chem. A. 2011, 115, 6472-6480.

(2) Herbst, E.; van Dishoeck, E. F. Annu. Rev. Astron. Astrophys. 2009, 47, 427-480.

(3) van Dishoeck, E. F.; Blake, G. A. Annu. Rev. Astron. Astrophys. 1998, 36, 317368.

(4) Ehrenfreund, P.; Charnley, S. B. Annu. Rev. Astron. Astrophys. 2000, 38, 427483.

(5) Woon, D. The Astrochymist, http://www.astrochymist.org/.

(6) Cami, J.; Bernard-Salas, J.; Peeters, E.; Malek, S. E. Science 2010, 329, 11801182.

(7) Kroto, H. W.; Heath, J. R.; O'Brien, S. C.; Curl, R. F.; Smalley, R. E. Nature 1985, 318, 162-163.

(8) Kroto, H. W. Philos. Trans. R. Soc. A 1988, 325, 405-421.

(9) Cheung, A. C.; Rank, D. M.; Townes, C. H.; Thornton, D. D.; Welch, W. J. Phys. Rev. Lett. 1968, 21, 1701.

(10) Cheung, A. C.; Rank, D. M.; Townes, C. H.; Thornton, D. D.; Welch, W. J. Nature 1969, 221, 626-628.

(11) Snyder, L. E.; Buhl, D.; Zuckerman, B.; Palmer, P. Phys. Rev. Lett. 1969, 22, 679.

(12) Herbst, E.; Klemperer, W. Astrophys. J. 1973, 185, 505-533. 
(13) Garrod, R. T.; Herbst, E. Astron. Astrophys. 2006, 457, 927-936.

(14) Garrod, R. T.; Widicus Weaver, S. L.; Herbst, E. Astrophys. J. 2008, 682, 283.

(15) Horn, A.; Mollendal, H.; Sekiguchi, O.; Uggerud, E.; Roberts, H.; Herbst, E.; Viggiano, E. E.; Fridgen, T. D. Astrophys. J. 2004, 611, 605.

(16) Bennett, C. J.; Kaiser, R. I. Astrophys. J. 2007, 661, 899.

(17) Oberg, K. I.; Garrod, R. T.; van Dishoeck, E. F.; Linnartz, H. Astron. Astrophys. 2009, 504, 891-913.

(18) Modica, P.; Palumbo, M. E. Astron. Astrophys. 2010, 519, A22.

(19) Blake, G. A.; Mundy, L. G.; Carlstrom, J. E.; Padin, S.; Scott, S. L.; Scoville, N. Z.; Woody, D. P. Astrophys. J. Lett. 1996, 472, L49.

(20) Zapata, L. A.; Schmid-Burgk, J.; Menten, K. M. Astron. Astrophys. 2010, 529, A24.

(21) Guélin, M.; Brouillet, N.; Cernicharo, J.; Combes, F.; Wooten, A. Astrophys. Space Sci. 2008, 313, 45-51.

(22) Friedel, D. N.; Snyder, L. E. Astrophys. J. 2008, 672, 962.

(23) Menten, K. M.; Reid, M. J. Astrophys. J. 1995, 445, L157-L160.

(24) Plambeck, R. L.; Wright, M. C. H.; Friedel, D. N.; Widicus Weaver, S. L.; Bolatto, A. D.; Pound, M. W.; Woody, D. P.; Lamb, J. W. Astrophys. J. 2009, 704, L25-L28.

(25) Reid, M. J.; Menten, K. M. Astrophys. J. 2007, 664, 950-955.

(26) The National Radio Astronomy Observatory is a facility of the National Science Foundation operated under cooperative agreement by Associated Unversities, Inc. 
(27) Support for CARMA construction was derived from the states of California, Illinois, and Maryland, the James S. McDonnell Foundation, the Gordon and Betty Moore Foundation, the Kenneth T. and Eileen L. Norris Foundation, the University of Chicago, the Associates of the California Institute of Technology, and the National Science Foundation. Ongoing CARMA development and operations are supported by the National Science Foundation under a cooperative agreement, and by the CARMA partner universities.

(28) Favre, C.; Despois, D.; Brouillet, N.; Baudry, A.; Combes, F.; Guélin, M.; Wooten, A.; Wlodarczak, G. Astron. Astrophys. 2011.

(29) Sutton, E. C.; Peng, R.; Danchi, W. C.; Jaminet, P. A.; Sandell, G.; Russell, A. P. G. Astrophys. J. Suppl. Ser. 1995, 97.

(30) Aikawa, Y.; Wakelam, V.; Garrod, R. T.; Herbst, E. Astrophys. J. 2008, 674, 9931005.

(31) Remijan, A. J.; Hollis, J. M. Astrophys. J. 2006, 640, 842-848.

(32) Blake, G. A.; Sutton, E. C.; Masson, C. R.; Phillips, T. G. Astrophys. J. 1987, $315,621-645$.

(33) Millar, T. J.; Herbst, E.; Charnley, S. B. Astrophys. J. 1991, 369, 147-156.

(34) Tholmann, D.; McCormick, A.; McMahon, T. B. J. Phys. Chem. 1994, 98, 11561160.

(35) Fridgen, T. D.; McMahon, T. B. J. Phys. Chem. A 2001, 105, 1011-1019.

(36) Ryzhov, V.; Yang, Y.-C.; Klippenstein, S. J.; Dunbar, R. C. J. Phys. Chem. A 1998, 102, 8865-8870. 
(37) Herbst, E.; DeFrees, D. J.; Talbi, D.; Pauzat, F.; Koch, W.; McLean, A. D. J. Chem. Phys. 1991, 94, 7842-7849.

(38) Herbst, E.; Yamashita, K. J. Chem. Soc., Faraday Trans. 1993, 89, 2175-2183.

(39) McCall, B. J.; Oka, T. Science 2000, 287, 1941-1942.

(40) Watson, W. D. Astrophys. J. 1973, 183, L17-L20.

(41) Mackay, G. I.; Hopkinson, A. C.; Bohme, D. K. J. Am. Chem. Soc. 1978, 100, 7460-7464.

(42) Freeman, C. G.; Harland, P. W.; McEwan, M. J. Aust. J. Chem. 1978, 31, 21572160.

(43) Mackay, G. I.; Tanner, S. D.; Hopkinson, A. C.; Bohme, D. K. Can. J. Chem. 1979, 57, 1518-1523.

(44) Feng, W. Y.; Lifshitz, C. J. Phys. Chem. 1994, 98, 3658-3663.

(45) Tiedemann, P. W.; Riveros, J. W. J. Am. Chem. Soc. 1974, 96, 185-189.

(46) Fileti, E. E.; De Oliveira, A. E.; Morgon, N. H.; Riveros, J. M. Int. J. Quantum Chem. 2011, 111, 1596-1606.

(47) Karpas, Z.; Meot-Ner, M. J. Phys. Chem. 1989, 93, 1859-1863.

(48) Meot-Ner, M.; Karpas, Z. J. Phys. Chem. 1986, 90, 2206-2210.

(49) Fridgen, T. D.; Keller, J. D.; McMahon, T. B. J. Phys. Chem. A 2001, 105, 38163824.

(50) Freeman, C. G.; Harland, P. W.; McEwan, M. J. International Journal or Mass Spectrometry and Ion Physics 1978, 28, 377-388.

(51) Huntress, W. T.; Bowers, M. T. Int. J. Mass Spectrom. Ion Phys. 1973, 12, 1-18. 
(52) Fiaux, A.; Smith, D. L.; Futrell, J. H. Int. J. Mass Spectrom. Ion Phys. 1976, 20, 223-235.

(53) Bouchoux, G.; Choret, N. Rapid Commun. Mass Spectrom. 1997, 11, 1799-1807.

(54) Peeters, Z.; Rodgers, S. D.; Charnley, S. B.; Schriver-Mazzuoli, L.; Schriver, A.; Keane, J. V.; Ehrenfreund, P. Astron. Astrophys. 2006, 445, 197-204.

(55) Neau, A.; Khalili, A. A.; Rosen, S.; Le Padellec, A.; Derkatch, A. M.; Shi, W.; Vikor, L.; Larsson, M.; Semaniak, J.; Thomas, R.; Nagard, M. B.; Andersson, K.; Danared, H.; Ugglas, M. a. J. Chem. Phys. 2000, 113, 1762-1770.

(56) Geppert, W. D.; Hamberg, M.; Thomas, R. D.; Osterdahl, F.; Hellberg, F.; Zhaunerchyk, V.; Ehlerding, A.; Millar, T. J.; Roberts, H.; Semaniak, J.; af Ugglas, M.; Kallberg, A.; Simonsson, A.; Kaminska, M.; Larsson, M. Faraday Discuss. 2006, 133, 177-190.

Hamberg, M.; Geppert, W. D.; Thomas, R. D.; Zhaunerchyk, V.; Osterdahl, F.; Ehlerding, A.; Kaminska, M.; Semaniak, J.; Ugglas, M. a.; Kollberg, A.; Paal, A.; Simonsson, A.; Larsson, M. Mol. Phys. 2007, 105, 899-906.

(58) Hamberg, M.; Osterdahl, F.; Thomas, R. D.; Zhaunerchyk, V.; Vigren, E.; Kaminska, M.; af Ugglas, M.; Kollberg, A.; Simonsson, A.; Paol, A.; Larsson, M.; Geppert, W. D. Astron. Astrophys. 2010, 514, A83.

(59) Vigren, E.; Hamberg, M.; Zhaunerchyk, V.; Kaminska, M.; Semaniak, J.; Larsson, M.; Thomas, R. D.; af Ugglas, M.; Kashperka, L.; Millar, T. J.; Walsh, C.; Roberts, H.; Geppert, W. D. Astrophys. J. 2010, 709, 1429-1434. 
(60) Goddard, J. D.; Yamaguchi, Y.; Schaefer III, H. F. J. Chem. Phys. 1992, 96, $1158-1166$.

(61) Glockler, G. J. Phys. Chem. 1959, 63, 828-832.

(62) Francisco, J. S. J. Am. Chem. Soc. 2003, 125, 10475-10480.

(63) Metcalfe, W. K.; Simmie, J. M.; Curran, H. J. J. Phys. Chem. A 2010, 114, 54785484.

(64) Hunter, E. P. L.; Lias, S. G. J. Phys. Chem. Ref. Data 1998, 27, 413-656.

(65) Senent, M. L.; Villa, M.; Melendez, F. J.; Dominguez-Gomez, R. Astrophys. J. 2005, 627, 567-576.

(66) Wennerstrom, H.; Forsen, S.; Roos, B. J. Phys. Chem. 1972, 76, 2430-2436.

(67) Fausto, R.; Teixeira-dias, J. J. C. J. Mol. Struct. THEOCHEM 1987, 150, 381389.

(68) Wiberg, K. B.; Laidig, K. E. J. Am. Chem. Soc. 1987, 109, 5935-5943.

(69) Peterson, M. R.; Csizmadia, I. G. J. Am. Chem. Soc. 1979, 101, 1076-1079.

(70) Belloche, A.; Garrod, R. T.; Muller, H. S. P.; Menten, K. M.; Comito, C.; Schilke, P. Astron. Astrophys. 2009, 499, 215-232.

(71) McCarthy, M. C.; Chen, W.; Travers, M. J.; Thaddeus, P. Astrophys. J. Suppl. Ser. 2000, 129, 611-623.

(72) Laas, J.; Garrod, R. T.; Herbst, E.; Widicus Weaver, S. L. Astrophys. J. 2011, 728,71 .

(73) Liu, S.-Y.; Girart, J. M.; Remijan, A. J.; Snyder, L. E. Astrophys. J. 2002, 576, $255-263$. 


\section{Chapter 5}

Laboratory Detection of trans-Methyl Formate and trans-gauche-Ethyl Formate, and Interstellar Detection of trans-Methyl Formate

\section{Introduction}

In the previous chapter, based on the spatial distributions of methyl formate and related molecules in the Orion Compact Ridge, two gas-phase ion-molecule reactions were proposed that could explain the observed anticorrelation in the abundances of methyl formate and formic acid in this region. Both reactions, but particularly the methyl cation transfer process, would be expected to produce an observable quantity of the trans conformational isomer of methyl formate. The interstellar detection of this species had not been previously anticipated due to the low population of trans-methyl formate at thermal equilibrium.

The existence of the trans conformer of methyl formate has been known for many years. The energy difference between the two conformers has been determined in solution using the temperature-drift technique, ${ }^{1}$ dynamic NMR spectroscopy, ${ }^{2,3}$ and infrared spectroscopy in an argon matrix. ${ }^{4,5}$ In the first experimental study of the rotational spectrum of methyl formate, ${ }^{6}$ this conformer was sought but no transitions were found. Based on the calculations of Senent et al., ${ }^{7}$ the abundance ratio between the two conformers at room temperature equilibrium is $\sim 30,000: 1$. The Stark-modulated room-temperature waveguide spectrometer employed in this study, therefore, did not have sufficient sensitivity to detect this isomer. The analogous conformers of ethyl 
formate in the trans ester conformation were also sought in a microwave spectroscopy study, ${ }^{8}$ but were not found for the same reason.

Based on the work presented in Chapter 4, the rotational spectrum at microwave frequencies $(<40 \mathrm{GHz})$ of trans-methyl formate was assigned using several microwave spectrometers, including chirped-pulse FTMW spectrometers. The spectrum of the corresponding conformer of ethyl formate was likewise studied. The assignment of these two species will be presented in this chapter. Additionally, based on the laboratory frequencies measured in this study, the first interstellar detection of trans-methyl formate, in the Sagittarius B2(N) molecular cloud, using the Green Bank Telescope (GBT), will be reported. Due to the large interconversion barrier between these two conformers, the observed relative abundances of the two species reflect conformer-specific kinetics, rather than their thermodynamic stability. The trans isomer is also observed to be considerably colder than cis in this source, suggesting that the two conformers may have different spatial distributions and production mechanisms. Possible explanations for this differentiation will be discussed.

\section{Experimental}

The laboratory microwave spectra of the trans-methyl and ethyl formates were measured on several pulsed-jet FTMW spectrometers: CP-FTMW spectrometers operating from 7-18.5 GHz and 25-40 GHz, and two cavity FTMW spectrometers, a

miniature cavity based on the NIST design ${ }^{9}$ and a cryogenically cooled spectrometer. ${ }^{10}$ The 25-40 GHz spectrometer will not be discussed in detail here, but a schematic of this 
spectrometer is shown in Figure 5.1. The most important difference from the lowerfrequency spectrometers discussed previously in this thesis is that after the generation of a 12.5-20.0 GHz chirped pulse (by mixing the output of the AWG with a $23.00 \mathrm{GHz}$ PDRO), an active frequency doubler is used to generate a $25-40 \mathrm{GHz}$ chirped pulse. Additionally, the molecular FIDs in this spectral region cannot be digitized directly as in the 7.0-18.5 GHz region, and so these signals are downconverted using the same 23.00 $\mathrm{GHz}$ oscillator to bring them into the spectral range of the $50 \mathrm{GS} / \mathrm{s}$ digitizer. Also, it should be noted that only two nozzles were used for this spectrometer design due to the lower broadcast directionality of the horn antennas used in this frequency range.

Samples of methyl formate (99\%, Aldrich) and ethyl formate (97\%, Aldrich) were diluted to $0.2 \%$ in neon carrier gas. Due to the low population of these species at a room temperature equilibrium, a pulsed discharge nozzle of the type previously described by McCarthy et al., ${ }^{11}$ operated with a voltage of approximately $800 \mathrm{~V}$, was used to increase the populations of these species in the supersonic jet by a factor of approximately 30. Due to the strong internal rotation effects in the spectra of these conformers, FTMW-MW double resonance measurements of the type described in Chapter 3 were used to confirm the spectral assignments reported here, and to locate new transitions. Finding new transitions was done by monitoring a known transition of the species, and scanning the double resonance microwave source in the region of interest. Double resonance sources with at least $100 \mathrm{~mW}$ of power from 6-60 GHz were employed. 


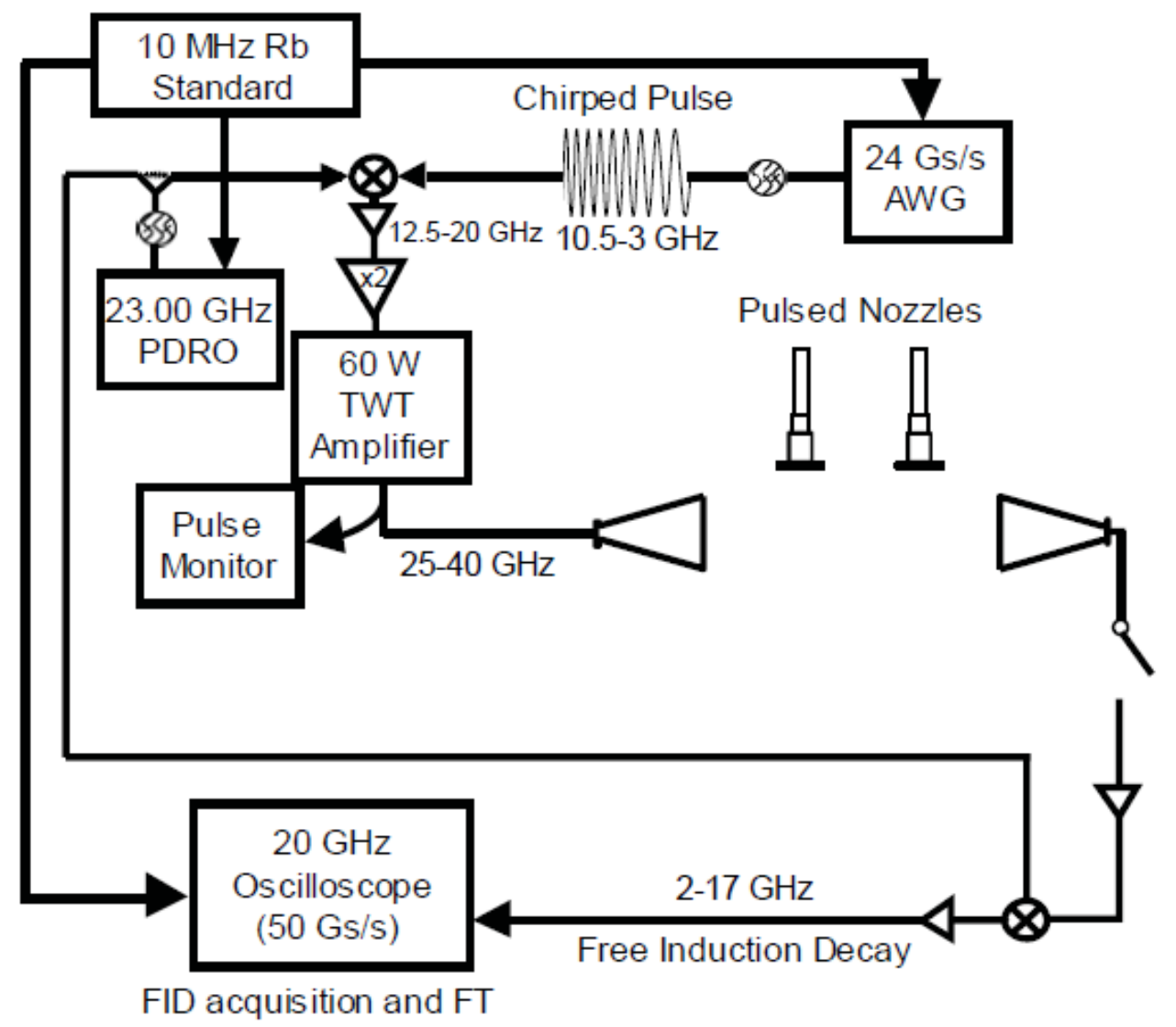

Figure 5.1. Schematic of the 25-40 GHz CP-FTMW spectrometer as used to detect the rotational spectra of trans-methyl formate and trans-gauche ethyl formate. 


\section{Laboratory Detection of trans-Methyl Formate}

A total of 33 transitions of the dominant $\left(\mathrm{H}^{12} \mathrm{C}^{16} \mathrm{O}^{16} \mathrm{O}^{12} \mathrm{CH}_{3}\right)$ isotopologue of trans-methyl formate were detected in this study, 24 of the $A$ symmetry species, and 9 of the $E$ symmetry species, with $a$ - and $b$-type selection rules. In addition, 13 transitions of each of the singly-substituted ${ }^{13} \mathrm{C}$ isotopologues were observed in natural abundance. All transitions up to $40 \mathrm{GHz}$ with appreciable intensity at pulsed-jet temperatures $\left(T_{\text {rot }} \sim 1 \mathrm{~K}\right)$ have been measured. The standard asymmetry top quantum numbers $J_{K a K c}$ are used to designate energy levels for the $A$ symmetry species ( $m=0$, where $m$ is the methyl torsion quantum number), but due to the strong coupling between the internal rotation of the methyl group and the overall rotation of the molecule, the symmetric rotor quantum numbers $J_{K}$, with a signed value of $K$, are used for the $E$ symmetry species $(m= \pm 1)$. The perturbation of the rotational energy levels due to methyl torsion/rotation coupling is illustrated in Figure 5.2. All of the transitions of trans-methyl formate observed in this study are presented in Table 5.1. It should be noted that the higher uncertainties of the transitions above $40 \mathrm{GHz}$ are due to the fact that these transitions were only measured using microwave-microwave double resonance, in which the linewidth (limited by the transform-limited linewidth of the double resonance pulse) is broader than the free induction decay linewidth. Representative CP-FTMW spectra of methyl formate, showing the intensity difference between the cis and trans conformers and the enhancement provided by the pulsed discharge nozzle, are presented in Figure 5.3.

The literature devoted to the analysis of the rotational spectra of asymmetric top 
(a)

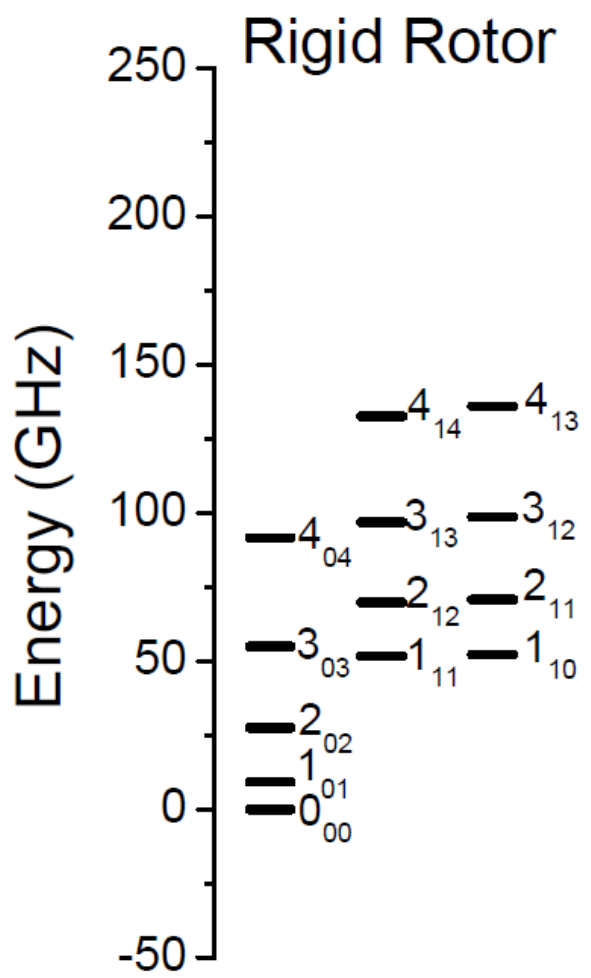

(b)

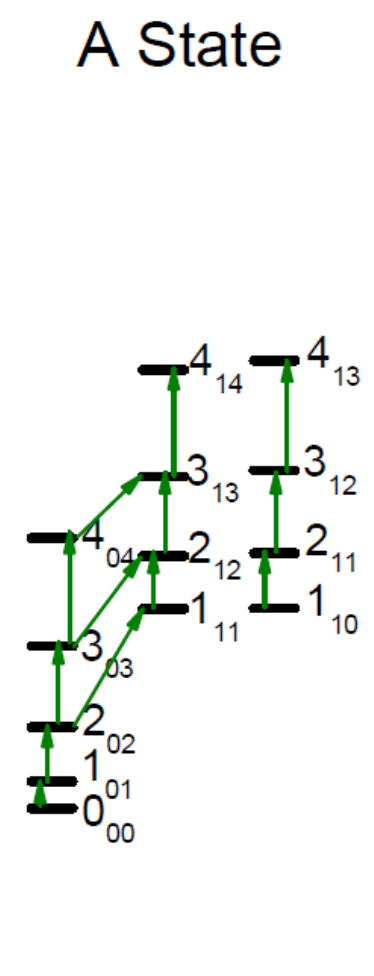

(c)

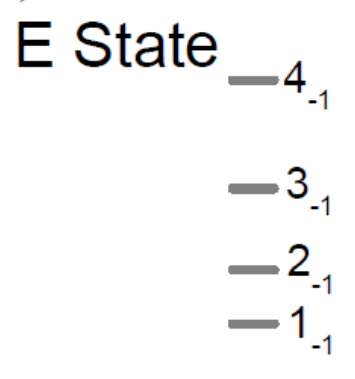

Figure 5.2. Energy levels for low-lying states of trans-methyl formate. Panel (A) shows the calculated energy levels assuming a pseudo-rigid rotor model (with no effects due to methyl internal rotation). Panel (B) shows the actual energy levels of the $A$ symmetry species (green arrows indicate measured transitions). The $K_{\mathrm{a}}=1$ states are shifted higher in energy relative to the $K_{\mathrm{a}}=0$ states than in the pseudo-rigid rotor. Panel (C) shows the energy levels of the $E$ symmetry species. The $K=1$ and -1 levels are moved in opposite directions relative to the $A$ species. The $K=-1$ states have not been measured in the present study, due to their high energy (and therefore low population in the supersonic expansion). 
Table 5.1. Rotational transitions of trans-methyl formate and its two singly-substituted ${ }^{13} \mathrm{C}$ isotopologues observed in the laboratory.

$\underline{A \text { symmetry species }}$

\begin{tabular}{ccccccccc}
\hline & & & & & $\mathrm{H}^{12} \mathrm{COO}^{12} \mathrm{CH}_{3}$ & $\mathrm{H}^{13} \mathrm{COO}^{12} \mathrm{CH}_{3}$ & $\mathrm{H}^{12} \mathrm{COO}^{13} \mathrm{CH}_{3}$ \\
$\mathrm{~J}^{\prime}$ & $\mathrm{K}_{\mathrm{a}}{ }^{\prime}$ & $\mathrm{K}_{\mathrm{c}}{ }^{\prime}$ & $\mathrm{J}^{\prime \prime}$ & $\mathrm{K}_{\mathrm{a}}{ }^{\prime \prime}$ & $\mathrm{K}_{\mathrm{c}}{ }^{\prime}$ & Frequency $(\mathrm{MHz})$ & Frequency $(\mathrm{MHz})$ & Frequency $(\mathrm{MHz})$ \\
\hline 7 & 0 & 7 & 6 & 1 & 6 & $8812.533(3)$ & -- & -- \\
1 & 0 & 1 & 0 & 0 & 0 & $9124.221(3)$ & $9091.497(5)$ & $8878.750(5)$ \\
7 & 1 & 6 & 7 & 1 & 7 & $9160.378(3)$ & -- & -- \\
4 & 1 & 4 & 5 & 0 & 5 & $11124.535(3)$ & -- & -- \\
8 & 1 & 7 & 8 & 1 & 8 & $11776.682(3)$ & -- & -- \\
9 & 1 & 8 & 9 & 1 & 9 & $14719.245(3)$ & -- & - \\
2 & 1 & 2 & 1 & 1 & 1 & $17921.508(3)$ & -- & -- \\
10 & 1 & 9 & 10 & 1 & 10 & $17987.583(3)$ & -- & $17756.242(5)$ \\
2 & 0 & 2 & 1 & 0 & 1 & $18247.038(3)$ & $18181.567(5)$ & - \\
2 & 1 & 1 & 1 & 1 & 0 & $18575.916(3)$ & -- & - \\
3 & 1 & 3 & 4 & 0 & 4 & $20877.496(3)$ & -- & $26169.865(10)$ \\
3 & 1 & 3 & 2 & 1 & 2 & $26881.342(3)$ & $26779.167(10)$ & - \\
3 & 0 & 3 & 2 & 0 & 2 & $27367.048(3)$ & $27268.729(10)$ & $26631.176(10)$ \\
3 & 1 & 2 & 2 & 1 & 1 & $27862.941(3)$ & $27768.743(10)$ & $27101.760(10)$ \\
2 & 1 & 2 & 3 & 0 & 3 & $30479.000(3)$ & -- & $34891.610(10)$ \\
4 & 1 & 4 & 3 & 1 & 3 & $35840.078(3)$ & $35703.788(10)$ & $35502.325(10)$ \\
4 & 0 & 4 & 3 & 0 & 3 & $36482.850(3)$ & $36351.539(10)$ & - \\
4 & 1 & 3 & 3 & 1 & 2 & $37148.834(3)$ & $37023.179(10)$ & $36134.080(10)$ \\
1 & 1 & 1 & 2 & 0 & 2 & $39924.543(3)$ & -- & - \\
1 & 1 & 0 & 1 & 0 & 1 & $58498.725(50)$ & -- & - \\
2 & 1 & 1 & 2 & 0 & 2 & $58827.710(50)$ & -- & - \\
3 & 1 & 2 & 3 & 0 & 3 & $59323.533(50)$ & -- & - \\
4 & 1 & 3 & 4 & 0 & 4 & $59989.491(50)$ & -- & - \\
5 & 1 & 4 & 5 & 0 & 5 & $60829.684(50)$ & -- & \\
\hline
\end{tabular}

$\underline{\text { E symmetry species }}$

\begin{tabular}{|c|c|c|c|c|c|c|}
\hline $\mathrm{J}^{\prime}$ & $\mathrm{K}^{\prime}$ & $\mathrm{J}^{\prime \prime}$ & $\mathrm{K}^{\prime \prime}$ & $\begin{array}{c}\mathrm{H}^{12} \mathrm{COO}^{12} \mathrm{CH}_{3} \\
\text { Frequency }(\mathrm{MHz})\end{array}$ & $\begin{array}{c}\mathrm{H}^{13} \mathrm{COO}^{12} \mathrm{CH}_{3} \\
\text { Frequency }(\mathrm{MHz})\end{array}$ & $\begin{array}{c}\mathrm{H}^{12} \mathrm{COO}^{13} \mathrm{CH}_{3} \\
\text { Frequency }(\mathrm{MHz})\end{array}$ \\
\hline 1 & 0 & 0 & 0 & $9207.427(3)$ & -- & -- \\
\hline 2 & 1 & 1 & 1 & $17820.962(3)$ & -- & -- \\
\hline 2 & 0 & 1 & 0 & $18367.848(3)$ & $18303.082(5)$ & $17872.392(5)$ \\
\hline 2 & 0 & 3 & 1 & $26053.738(3)$ & -- & -- \\
\hline 3 & 1 & 2 & 1 & $26750.960(3)$ & $26641.579(10)$ & - \\
\hline 3 & 0 & 2 & 0 & $27440.992(3)$ & $27338.687(10)$ & 26710.491(10) \\
\hline 1 & 0 & 2 & 1 & $34436.849(3)$ & -- & -- \\
\hline 4 & 1 & 3 & 1 & $35701.086(3)$ & $35556.827(10)$ & $34767.037(10)$ \\
\hline 4 & 0 & 3 & 0 & $36406.672(3)$ & $36263.248(10)$ & $35451.242(10)$ \\
\hline
\end{tabular}



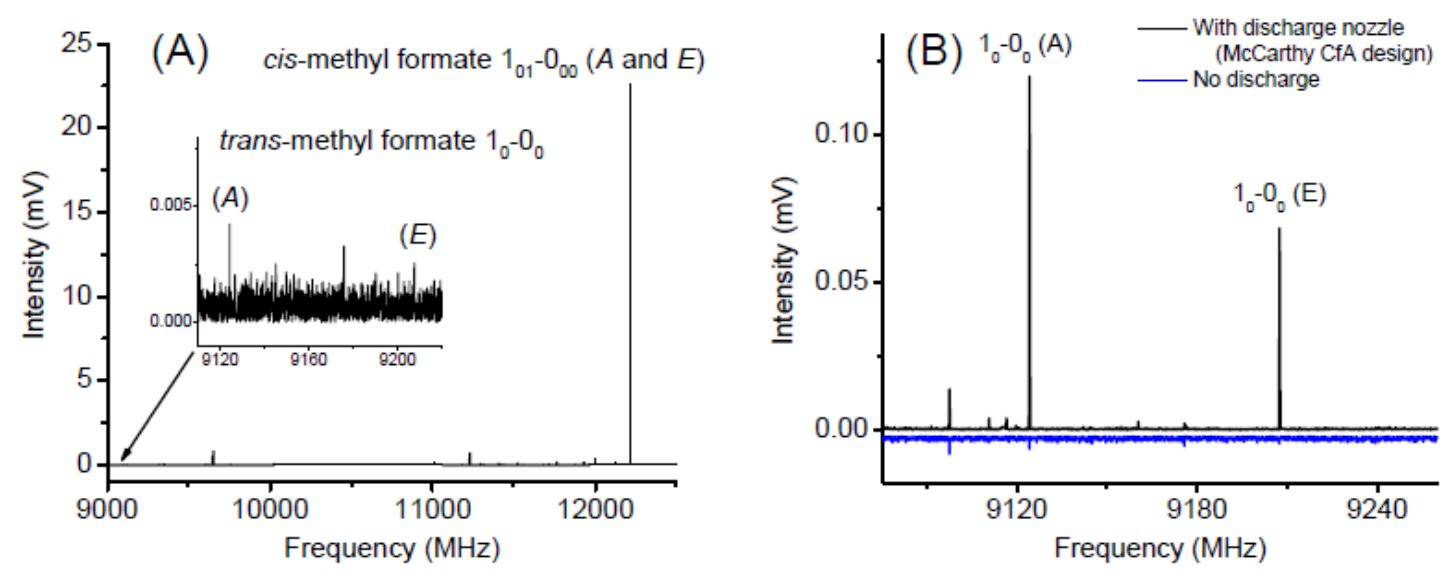

Figure 5.3. CP-FTMW spectra of methyl formate. Panel A: Spectrum with no discharge (31,000 signal averages, 90 minutes data collection time) showing the relative abundances of cis- and trans-methyl formate. Panel B: Comparison of spectra with and without a discharge nozzle. 
molecules with low-barrier methyl internal rotors is extensive. ${ }^{12}$ Several freely available programs are available to fit these spectra, using several different axis systems to minimize particular off-diagonal Hamiltonian terms. For this study the BELGI program was used, ${ }^{13}$ which employs the rho-axis method (RAM). Generally the internal rotation Hamiltonian can be written as

$$
\widehat{H}=\widehat{H}_{r}+\widehat{H}_{t r}+\widehat{H}_{t}
$$

The overall rotation part $\left(H_{r}\right)$ contains the $P_{a}^{2}, P_{b}^{2}$, and $P_{c}^{2}$ overall rotation angular momentum terms and normal centrifugal distortion constants. The methyl torsion component $\left(H_{t}\right)$ contains the electronic potential term $V_{3}$ and a term due to the momentum of the methyl top, which has an internal rotation constant $F$ which describes its inertia. The torsion/rotation component, $H_{t r}$, contains coupling terms due to the interaction between the angular momentum of the methyl top, $P_{\alpha}$, and the three angular momenta of the overall rotation. The idea behind the RAM method is to rotate the axis system away from the principal axis system by an angle $\theta_{\mathrm{RAM}}$ such that only one of these three coupling terms remains. In both conformers of methyl formate, due to their $C_{s}$ symmetry, this rotation is in the $a-b$ plane. The new RAM axis system is not the principal axis system of the molecule, and so an angular momentum term of the form $P_{a} P_{b}+P_{b} P_{a}$ (with inertial constant $D_{a b}$ ) exists due to an off-diagonal term in the moment of inertia tensor. The axes in the RAM system are typically still referred to as $a, b$, and $c$, and care must be taken to differentiate these axes from the principal axes. The rotational constants are referred to here as $A_{\mathrm{RAM}}, B_{\mathrm{RAM}}$, and $C_{\mathrm{RAM}}$ to clarify that these are not strictly the normal $A, B$, and $C$. (However, because the rotation away from the principal axis 
system is only the $a-b$ direction, for this molecule $C_{\mathrm{RAM}}=C$ ). The rho-axis method offers faster convergence than systems based on leaving the axis system as the principal axis system of the molecule because of the reduction in torsion-rotation coupling. The BELGI program in particular has demonstrated great usefulness in the fitting of lowbarrier methyl tops, such as in the spectra of acetamide $\left(V_{3}=25 \mathrm{~cm}^{-1}\right)^{14}$ and 2-butynol $\left(V_{3}\right.$ $\left.=7 \mathrm{~cm}^{-1}\right) .{ }^{15}$

As the supersonic expansion cooled out all torsionally excited states in these spectrometers, there is some indeterminacy in the Hamiltonian parameters presented here. In particular, when fitting only the ground torsional state, there is a strong correlation between the internal rotation constant, $F$, and the methyl rotation barrier height, $V_{3}$; therefore, the value of $F$ was fixed to its $a b$ initio value. This was done because the value of $F$ depends on only the mass of the internal rotor (three hydrogen atoms) and its orientation in the molecule, which is likely to be more accurately calculated by electronic structure theory than the electronic properties of the barrier. The derived rotation and internal rotation parameters than result from the fit of the spectra of trans-methyl formate are presented in Table 5.2, compared to values calculated by electronic structure theory. For each of the three isotopologues, the fit of the observed transitions is to experimental uncertainty. Many of the parameters derived from the normal species fit were held constant in the fits of the ${ }^{13} \mathrm{C}$ isotopologues, particularly the distortion constants and other higher-order parameters that are not expected to change significantly upon isotopic substitution. 
Table 5.2. Hamiltonian fit parameters of trans-methyl formate and its singly-substituted ${ }^{13} \mathrm{C}$ isotopologues.

\begin{tabular}{|c|c|c|c|c|c|}
\hline Parameter & Operator & $\begin{array}{l}\text { Normal } \\
\text { Species }\end{array}$ & $A b$ initio $^{\mathrm{a}}$ & $\mathrm{H}^{13} \mathrm{COOCH}_{3}$ & $\mathrm{HCOO}^{13} \mathrm{CH}_{3}$ \\
\hline$V_{3}\left(\mathrm{~cm}^{-1}\right)$ & $1 / 2(1-\cos 3 \gamma)$ & $12.955(17)$ & 22.5 & $12.955^{\mathrm{c}}$ & $12.955^{\mathrm{c}}$ \\
\hline$F\left(\mathrm{~cm}^{-1}\right)$ & $P_{\gamma}^{2}$ & $7.04^{\mathrm{b}}$ & 7.04 & $7.04^{c}$ & $7.04^{\mathrm{c}}$ \\
\hline$\rho$ (unitless) & $P_{\gamma} P_{\mathrm{a}}$ & $0.27347(3)$ & 0.274 & $0.26922(6)$ & $0.27343(7)$ \\
\hline$A_{\mathrm{RAM}}(\mathrm{MHz})$ & $P_{\mathrm{a}}^{2}$ & $47390(3)$ & 47908.11 & $46908(6)$ & $47306(6)$ \\
\hline$B_{\text {RAM }}(\mathrm{MHz})$ & $P_{\mathrm{b}}^{2}$ & $4784.25(11)$ & 4740.59 & $4770.339(3)$ & $4649.5182(26)$ \\
\hline$C_{\mathrm{RAM}}(\mathrm{MHz})$ & $P_{\mathrm{c}}^{2}$ & $4398.490(4)$ & 4368.00 & $4380.8047(14)$ & $4284.0389(13)$ \\
\hline$D_{\mathrm{ab}}(\mathrm{MHz})$ & $P_{\mathrm{a}} P_{\mathrm{b}}+P_{\mathrm{b}} P_{\mathrm{a}}$ & $1250(4)$ & 1812.44 & $1252.2(15)$ & $1203.8(14)$ \\
\hline$d_{\mathrm{ab}}(\mathrm{MHz})$ & $\begin{array}{c}(1-\cos 3 \gamma) \\
\left(P_{\mathrm{a}} P_{\mathrm{b}}+\right. \\
\left.P_{\mathrm{b}} P_{\mathrm{a}}\right)\end{array}$ & $661(4)$ & & $661.9(17)$ & $647.8(17)$ \\
\hline$G_{v}(\mathrm{MHz})$ & $P^{2} P_{\gamma}^{2}$ & $1.27(5)$ & & $1.27^{\mathrm{c}}$ & $1.27^{\mathrm{c}}$ \\
\hline$\Delta_{a b}(\mathrm{MHz})$ & $\begin{array}{c}P_{\gamma}^{2}\left(P_{\mathrm{a}} P_{\mathrm{b}}+\right. \\
\left.P_{\mathrm{b}} P_{\mathrm{a}}\right)\end{array}$ & $15.2(17)$ & & $15.2^{\mathrm{c}}$ & $15.2^{\mathrm{c}}$ \\
\hline$\Delta_{J}(\mathrm{kHz})$ & $-P^{4}$ & $1.102(9)$ & & $1.102^{\mathrm{c}}$ & $1.102^{\mathrm{c}}$ \\
\hline$\Delta_{J K}(\mathrm{kHz})$ & $-P^{2} P_{\mathrm{a}}^{2}$ & $-117.6(18)$ & & $-117.6^{c}$ & $-117.6^{c}$ \\
\hline$\delta_{J}(\mathrm{kHz})$ & $\begin{array}{c}-2 P^{2}\left(P_{\mathrm{b}}^{2}-\right. \\
\left.P_{\mathrm{c}}^{2}\right)\end{array}$ & $0.0531(11)$ & & $0.0531^{\mathrm{c}}$ & $0.0531^{\mathrm{c}}$ \\
\hline$N_{\text {lines }}$ & & 33 & & 13 & 13 \\
\hline$N_{\text {parameters }}$ & & 12 & & 6 & 6 \\
\hline
\end{tabular}

${ }^{\mathrm{a}}$ Calculated at an MP2/6-311++G(d,p) level of theory.

${ }^{\mathrm{b}}$ Fixed to its $a b$ initio value.

${ }^{c}$ Fixed to normal species value. 
It should be noted that due to the low number of transitions measured in this study, the number of parameters fit (12) is fairly large considering the limited data set. In particular, the number of $E$ symmetry species transitions is very small, and the internal rotor effects are considerable. An effective rigid-rotor fit of the $A$ symmetry species required a total of 6 parameters (the three rotational constants and three quartic centrifugal distortion constants $\Delta_{J}, \Delta_{J K}$, and $\delta_{J}$ ) to fit the $24 A$-species transitions to experimental uncertainty. In order to fit the $9 E$ symmetry species transitions along with the $A, 6$ new parameters must be added to the fit. For this reason, one must be extremely careful in extrapolating this fit to higher frequencies for the $E$ species. To guide searches at millimeter-wave frequencies, in Table 5.3 the results of the effective rigid rotor fit to the $A$ symmetry species alone are presented. These Hamiltonian parameters are likely to be more reliable in predicting the frequencies of higher-frequency $A$ species transitions, at least for $K_{a}=0$ and 1 transitions (at higher $K_{a}$ it may be unreliable because no transitions with $K_{a} \geq 2$ have yet been measured). It can also be seen in Table 5.3 that the effective $A$ rotational constant of this fit is far higher than the fit value in Table 5.2, the effect of the internal rotor perturbation that is reflected in the shift of the $K_{a}=1$ states to higher energy in Figure 5.2.

From the derived rotational constants of the three isotopologues, substitution coordinates for the two carbon atoms in trans-methyl formate can be derived, serving as further confirmation that the assigned species is trans-methyl formate. The RAM inverse inertial tensor derived from the Hamiltonian fits in Table 5.2 were diagonalized to derive 
Table 5.3. Fit Hamiltonian parameters for an effective rigid-rotor fit to the $A$ symmetry species of trans-methyl formate.

\begin{tabular}{cc}
\hline Parameter & Value \\
\hline$A(\mathrm{MHz})$ & $62897.147(5)$ \\
$B(\mathrm{MHz})$ & $4725.7172(5)$ \\
$C(\mathrm{MHz})$ & $4398.5091(5)$ \\
$\Delta_{\mathrm{J}}(\mathrm{kHz})$ & $1.152(11)$ \\
$\Delta_{\mathrm{JK}}(\mathrm{kHz})$ & $-74.61(17)$ \\
$\Delta_{\mathrm{K}}(\mathrm{kHz})$ & $0.1099(4)$ \\
$N_{\text {lines }}$ & 24 \\
\hline
\end{tabular}


the moments of inertia of the molecule in the principal axis system, from which Kraitchman equations were applied to derive the carbon atoms in the principal axis system. In Table 5.4, these substitution coordinates are compared to the ab initio calculated positions of the two carbon atoms, showing extremely good agreement. This direct structural information serves as additional, definitive proof of the structural identity of the assigned spectrum.

From the relative intensity difference between cis and trans methyl formate without the pulsed discharge nozzle applied, taking into account the dipole moment factor and the rotational partition functions of the two species, the energy difference between the two conformers can be estimated if it is assumed that no conformational cooling occurs in the supersonic expansion. As Ruoff et al. ${ }^{16}$ showed, conformational relaxation typically only occurs in supersonic jets when the barrier to relaxation is $400 \mathrm{~cm}^{-1}(575 \mathrm{~K})$ or less. By comparison of the intensities of the $1_{01}-0_{00} A$ species transitions of the two conformers in the CP-FTMW spectrometer, using values of $\mu_{\mathrm{a}}=1.63$ debye for the cis conformer ${ }^{6}$ and $\mu_{\mathrm{a}}=4.23$ debye (calculated at an MP2/6-311++G(d,p) level of theory) for the trans conformer, a population difference of 22,000:1 (cis:trans) is estimated at room temperature, corresponding to an energy difference of $2060 \mathrm{~cm}^{-1}$, or $2980 \mathrm{~K}$. This is in good agreement with electronic structure calculations, both those presented by Senent et al. ${ }^{7}$ and in this work.

The threefold methyl potential barrier $V_{3}$ of trans-methyl formate determined in this study $\left(12.955 \mathrm{~cm}^{-1}\right)$ is considerably lower than that of cis-methyl formate 
Table 5.4. Derived substitution coordinates ${ }^{\mathrm{a}}$ of the carbon atoms of trans-methyl formate.

\begin{tabular}{ccccc}
\hline & \multicolumn{2}{c}{ Substitution Coordinates } & \multicolumn{2}{c}{ Ab initio } \\
& $|a|$ & $|b|$ & $a$ & $b$ \\
\hline $\mathrm{C}_{1}$ & $0.598(3)$ & $0.334(5)$ & 0.599 & 0.356 \\
$\mathrm{C}_{5}$ & $1.7578(10)$ & $0.143(12)$ & -1.778 & 0.149 \\
\hline
\end{tabular}

${ }^{\mathrm{a}}$ All coordinates are reported in angstroms. 
$\left(379.4 \mathrm{~cm}^{-1}\right) .{ }^{17}$ While this report presents the first experimental determination of this barrier, this molecule has been studied computationally, so this dramatic difference in methyl potential barriers has been previously discussed. Wiberg et al. ${ }^{18}$ compared the calculated methyl rotation barriers of cis- and trans-methyl formate to those of structurally related molecules, and concluded that the methyl $\mathrm{V}_{3}$ barrier observed for cismethyl formate is due to repulsion between the carbonyl oxygen atom and the methyl proton when the two are eclipsed, leading to a strong preference for a staggered geometry (see Figure 4.7). In the trans form, however, the carbonyl oxygen is not in close proximity to the methyl protons, and this effect vanishes, resulting in a very small barrier to methyl internal rotation.

\section{Laboratory Detection of trans-gauche Ethyl Formate}

a) Potential energy surface of ethyl formate

The potential energy surface of ethyl formate is presented in Figure 5.4. This molecule has two coordinates of conformational flexibility, the O-C-O-C ester dihedral angle as in methyl formate, and the $\mathrm{C}-\mathrm{O}-\mathrm{C}-\mathrm{C}$ dihedral that sets the orientation of the terminal methyl group. As in methyl formate, the stable conformations have ester dihedral angles of either $0^{\circ}$ or $180^{\circ}$. The configurations with the ester in its cis orientation are more stable than those in the trans orientation by $\sim 20 \mathrm{~kJ} / \mathrm{mol}$, while the transition state for isomerizing between cis and trans conformers is $57.1 \mathrm{~kJ} / \mathrm{mol}$. It should be noted that these barriers are very similar to those of methyl formate. 


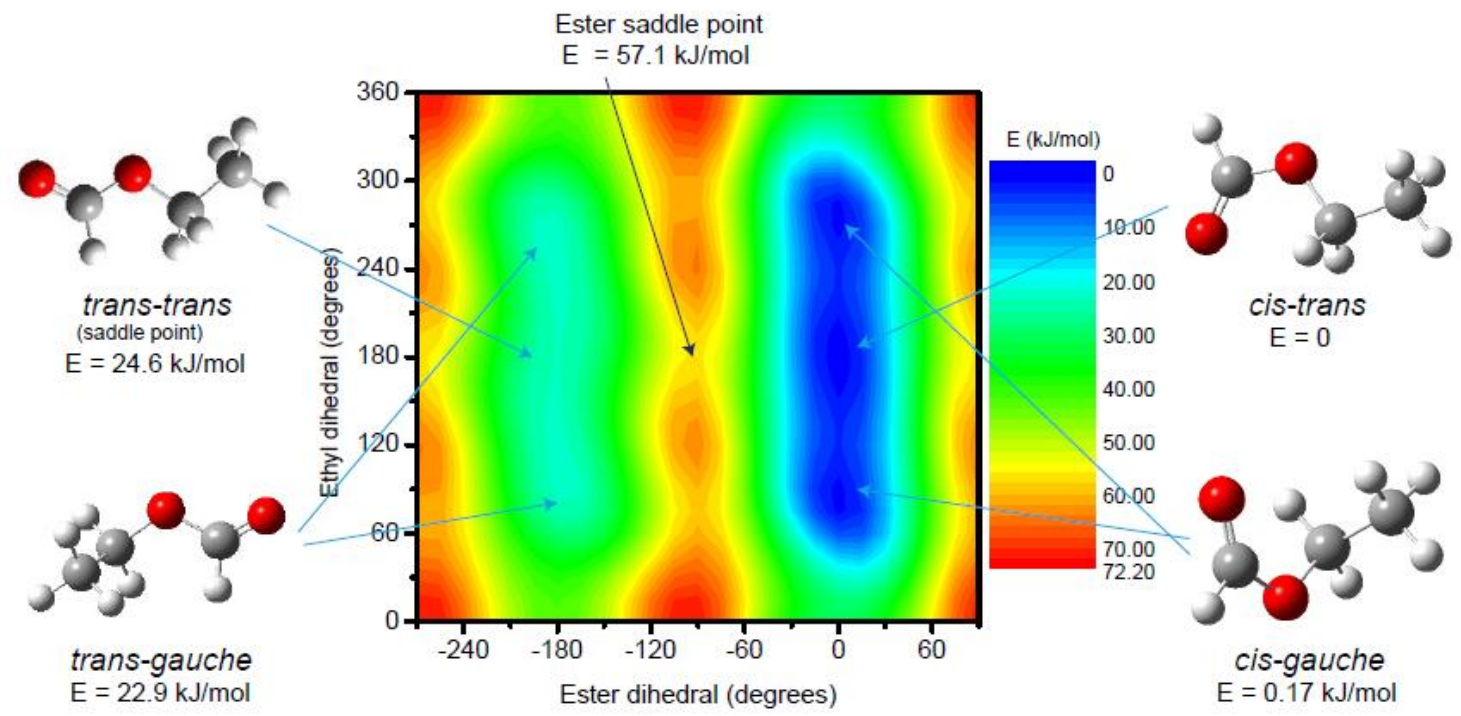

Figure 5.4. Two-dimensional potential energy surface of ethyl formate, calculated at an MP2/6-31+G(d,p) level of theory. The two dihedral angles of interest were scanned in 15 degree steps (576 steps in total), and on each step all of the other internal coordinates of the molecule were allowed to relax to their lowest-energy configuration. The structures and energies of the lowest-energy stationary points on the surface are indicated. 
In Figure 5.5, one-dimensional slices of this potential energy surface are shown to better display the energy barriers for moving along the ethyl coordinate. Conformers of ethyl formate are referred to by the orientation of the ester dihedral followed by the dihedral. For the cis ester orientation, the ethyl group can take on a trans, gauche ${ }^{+}$, or gauche $e^{-}$orientation. The gauche $e^{+}$and gauche conformers are mirror images of each other and therefore have identical rotational spectra. The two conformers are very near in energy, with the calculated energy of the cis-trans slightly lower. It should be noted that in Figure 5.5, the cis-gauche appears to be lower in energy, but this is due to the finite step size $\left(15^{\circ}\right)$ of the potential energy surface scan. The energy differences listed on Figure 5.4 are the energies that result from full geometry optimizations, with zero-point vibrational energy corrections. In the first microwave study of ethyl formate, ${ }^{8}$ where the cis-trans and cis-gauche conformer were assigned, the energy difference between these conformers was determined experimentally from relative intensity measurements to be $0.78(25) \mathrm{kJ} / \mathrm{mol}$, with the cis-trans conformer being lower in energy. The cis-trans and cis-gauche conformers were fit to rigid rotor spectra, with no internal rotation splitting (the calculated methyl $V_{3}$ barriers are greater than $1000 \mathrm{~cm}^{-1}$, so this is expected) or tunneling between conformers. A subsequent study of these two conformers in the millimeter wave region ${ }^{19}$ has likewise not found any evidence for internal motion splitting.

For the trans ester, the only stable orientation of the ethyl group is calculated to be gauche; the trans-trans is calculated to be a saddle point. As in the 


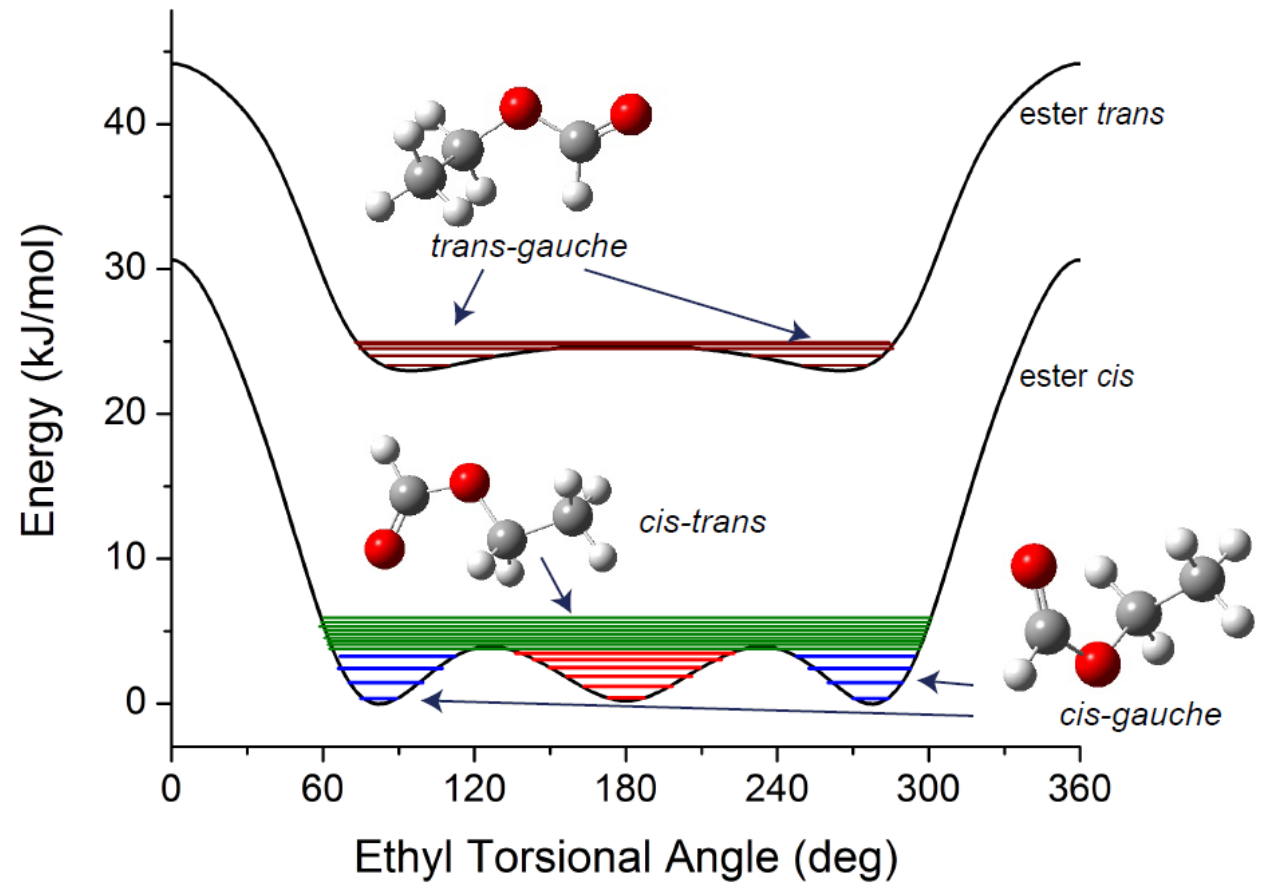

Figure 5.5. One-dimensional slices of the two-dimensional potential energy surface from Figure 5.4. 
cis configuration, there are two equivalent conformations of the gauche ethyl conformer. However, it should be noted from Figure 5.5 that the barrier to isomerization between the equivalent trans-gauche wells has a lower barrier (calculated to be $1.7 \mathrm{~kJ} / \mathrm{mol}$, or $140 \mathrm{~cm}^{-1}$ ) than the corresponding isomerization between the cis-gauche wells $(3.6 \mathrm{~kJ} / \mathrm{mol})$, which leads to splitting in the spectrum of the trans-gauche isomer due to tunneling between the two equivalent structures.

b) Microwave spectrum and Hamiltonian of trans-gauche-ethyl formate

A total of 79 transitions of trans-gauche ethyl formate were measured in this study; the frequencies of these transitions are presented in Tables 5.5 and 5.6. The transitions are grouped by their $K_{a}$ quantum numbers. For each of the $a$-type transitions, two closely spaced transitions were observed, while for the $c$-type transitions, a pair of lines were seen split by roughly $20 \mathrm{MHz}$. Tunneling between the two equivalent transgauche minima splits the spectrum into symmetric $\left(0^{+}\right)$and antisymmetric $\left(0^{-}\right)$states. Similar to the selection rules in place in the $(\mathrm{HCOOH})_{3}-\mathrm{H}_{2} \mathrm{O}$ complex discussed in Chapter 3 , because the tunneling motion is through the $a$ - $b$ principal axis plane, $a$-type transitions have selection rules of $0^{+} \leftarrow 0^{+}$and $0^{-} \leftarrow 0^{-}$, while $c$-type transitions have selection rules of $0^{-} \leftarrow 0^{+}$and $0^{+} \leftarrow 0^{-}$. Most of the assignments of the closely spaced $a$ type transitions to the $0^{+}$and $0^{-}$torsional states were made using closed loops involving two $a$-type and two $c$-type transitions.

CP-FTMW spectra of ethyl formate with the discharge nozzle activated, using the 
Table 5.5. Pure rotational (a-type) transitions of trans-gauche ethyl formate measured in the laboratory.

\begin{tabular}{|c|c|c|c|c|c|c|c|c|}
\hline $\mathrm{J}^{\prime}$ & $\mathrm{K}_{\mathrm{a}}{ }^{\prime}$ & $\mathrm{K}_{\mathrm{c}}{ }^{\prime}$ & $\mathrm{J}^{\prime \prime}$ & $\mathrm{K}_{\mathrm{a}}^{\prime \prime}$ & $\mathrm{K}_{\mathrm{c}}^{\prime \prime}$ & $\begin{array}{c}v\left(0^{+}\right) \\
(\mathrm{MHz})\end{array}$ & $\begin{array}{c}v\left(0^{-}\right) \\
(\mathrm{MHz})\end{array}$ & $\begin{array}{c}v\left(0^{-}\right)-v\left(0^{+}\right) \\
(\mathrm{MHz})\end{array}$ \\
\hline \multicolumn{9}{|c|}{$K_{a}=0$} \\
\hline 2 & 0 & 2 & 1 & 0 & 1 & $10368.483(3)$ & $10368.554(3)$ & 0.071 \\
\hline 3 & 0 & 3 & 2 & 0 & 2 & $15550.666(3)$ & $15550.769(3)$ & 0.103 \\
\hline 4 & 0 & 4 & 3 & 0 & 3 & $20730.381(3)$ & $20730.515(3)$ & 0.134 \\
\hline 5 & 0 & 5 & 4 & 0 & 4 & $25906.815(3)$ & 25906.981(3) & 0.166 \\
\hline 6 & 0 & 6 & 5 & 0 & 5 & $31079.170(3)$ & $31079.366(3)$ & 0.196 \\
\hline 7 & 0 & 7 & 6 & 0 & 6 & $36246.667(3)$ & $36246.890(3)$ & 0.223 \\
\hline \multicolumn{9}{|c|}{$K_{a}=1$} \\
\hline 3 & 1 & 3 & 2 & 1 & 2 & $15373.050(3)$ & $15373.081(3)$ & 0.031 \\
\hline 4 & 1 & 4 & 3 & 1 & 3 & $20496.115(3)$ & $20496.160(3)$ & 0.045 \\
\hline 5 & 1 & 5 & 4 & 1 & 4 & $25618.264(3)$ & $25618.323(3)$ & 0.059 \\
\hline 6 & 1 & 6 & 5 & 1 & 5 & $30739.226(3)$ & $30739.296(3)$ & 0.070 \\
\hline 7 & 1 & 7 & 6 & 1 & 6 & $35858.767(3)$ & $35858.852(3)$ & 0.085 \\
\hline \multicolumn{9}{|c|}{$K_{a}=-1$} \\
\hline 2 & 1 & 1 & 1 & 1 & 0 & $10489.762(3)$ & $10489.721(3)$ & -0.041 \\
\hline 3 & 1 & 2 & 2 & 1 & 1 & $15734.600(3)$ & $15734.567(3)$ & -0.033 \\
\hline 4 & 1 & 3 & 3 & 1 & 2 & $20978.213(3)$ & $20978.172(3)$ & -0.041 \\
\hline 5 & 1 & 4 & 4 & 1 & 3 & $26220.576(3)$ & $26220.523(3)$ & -0.053 \\
\hline 6 & 1 & 5 & 5 & 1 & 4 & $31461.422(3)$ & $31461.356(3)$ & -0.066 \\
\hline 7 & 1 & 6 & 6 & 1 & 5 & $36700.447(3)$ & $36700.370(3)$ & -0.077 \\
\hline \multicolumn{9}{|c|}{$\left|K_{a}\right|=2^{\mathrm{a}}$} \\
\hline$\frac{1}{5}$ & 2 & 4 & 4 & 2 & 3 & $25924.058(3)$ & $25923.419(3)$ & -0.639 \\
\hline 5 & 2 & 3 & 4 & 2 & 2 & $25939.475(3)$ & 25938.823(3) & -0.652 \\
\hline 6 & 2 & 5 & 5 & 2 & 4 & $31107.039(3)$ & $31106.556(3)$ & -0.483 \\
\hline 6 & 2 & 4 & 4 & 2 & 3 & $31132.330(3)$ & $31131.869(3)$ & -0.461 \\
\hline 7 & 2 & 6 & 6 & 2 & 5 & $36288.074(3)$ & $36287.804(3)$ & -0.270 \\
\hline 7 & 2 & 5 & 6 & 2 & 4 & $36328.909(3)$ & $36328.677(3)$ & -0.232 \\
\hline \multicolumn{9}{|c|}{$\left|K_{a}\right|=3^{\mathrm{b}}$} \\
\hline 5 & 3 & 3 & 4 & 3 & 2 & $25933.274(3)$ & (blend) & \\
\hline 5 & 3 & 2 & 4 & 3 & 1 & (blend) & (blend) & \\
\hline 6 & 3 & 4 & 5 & 3 & 3 & $31119.983(3)$ & (blend) & \\
\hline 6 & 3 & 3 & 5 & 3 & 2 & (blend) & (blend) & \\
\hline \multicolumn{9}{|c|}{$\left|K_{a}\right|=4^{\mathrm{b}}$} \\
\hline 6 & 4 & 3 & 5 & 4 & 2 & $31126.888(3)$ & (blend) & \\
\hline 6 & 4 & 2 & 5 & 4 & 1 & (blend) & (blend) & \\
\hline 7 & 4 & 4 & 6 & 4 & 3 & $36314.135(3)$ & (blend) & \\
\hline 7 & 4 & 3 & 6 & 4 & 2 & (blend) & (blend) & \\
\hline
\end{tabular}

${ }^{a}$ Due to the residual error in the fit of this species when including $K_{a}=2$ transitions, the assignment of these transitions to $0^{+}$or $0^{-}$is tentative.

${ }^{\mathrm{b}}$ For the $K_{a}=3$ and 4 transitions, the four lines for each $J$ (two values of $K_{c}$, and the two symmetry species) are unresolved. 
Table 5.6. Tunneling-rotation (c-type) transitions of trans-gauche ethyl formate measured in the laboratory.

\begin{tabular}{|c|c|c|c|c|c|c|c|c|}
\hline $\mathrm{J}^{\prime}$ & $\mathrm{K}_{\mathrm{a}}^{\prime}$ & $\mathrm{K}_{\mathrm{c}}^{\prime}$ & $\mathrm{J}^{\prime \prime}$ & $\mathrm{K}_{\mathrm{a}}^{\prime \prime}$ & $\mathrm{K}_{\mathrm{c}}^{\prime \prime}$ & $\begin{array}{l}v\left(0^{+} \leftarrow 0^{-}\right) \\
(\mathrm{MHz})\end{array}$ & $\begin{array}{l}v\left(0^{-} \leftarrow 0^{+}\right) \\
(\mathrm{MHz})\end{array}$ & $\begin{array}{c}v\left(0^{-} \leftarrow 0^{+}\right)-v\left(0^{+} \leftarrow 0^{-}\right) \\
(\mathrm{MHz})\end{array}$ \\
\hline \multicolumn{9}{|c|}{$K_{a}=1 \leftarrow 0(R$-branch $)$} \\
\hline 1 & 1 & 0 & 0 & 0 & 0 & $20035.087(3)$ & $20054.361(3)$ & 19.274 \\
\hline 2 & 1 & 1 & 1 & 0 & 1 & $25340.160(3)$ & $25359.424(3)$ & 19.264 \\
\hline 3 & 1 & 2 & 2 & 0 & 2 & $30706.207(3)$ & $30725.506(3)$ & 19.299 \\
\hline 4 & 1 & 3 & 3 & 0 & 3 & $36133.653(3)$ & $36153.012(3)$ & 19.359 \\
\hline \multicolumn{9}{|c|}{$K_{a}=1 \leftarrow 0(Q$-branch $)$} \\
\hline 1 & 1 & 1 & 1 & 0 & 1 & $14728.002(3)$ & $14747.330(3)$ & 19.328 \\
\hline 2 & 1 & 2 & 2 & 0 & 2 & $14609.040(3)$ & $14628.437(3)$ & 19.397 \\
\hline 3 & 1 & 3 & 3 & 0 & 3 & $14431.320(3)$ & $14450.851(3)$ & 19.531 \\
\hline 4 & 1 & 4 & 4 & 0 & 4 & $14196.919(3)$ & $14216.632(3)$ & 19.713 \\
\hline 5 & 1 & 5 & 5 & 0 & 5 & $13908.202(3)$ & $13928.139(3)$ & 19.937 \\
\hline 6 & 1 & 6 & 6 & 0 & 6 & $13568.062(3)$ & $13588.265(3)$ & 20.203 \\
\hline 7 & 1 & 7 & 7 & 0 & 7 & $13179.939(3)$ & $13200.450(3)$ & 20.511 \\
\hline
\end{tabular}


7.0-18.5 GHz spectrometer, are presented in Figure 5.6. Panel (A) shows the full view of the spectrum, which is dominated by the previously assigned conformers. Expanded views of the strong transitions of the trans-gauche conformer in this frequency range are presented in panels (B) and (C). The low intensities of the transitions of this conformer compared to the two stronger conformers explains why it was not detected in the previous studies of the rotational spectrum of ethyl formate; even with the discharge nozzle, the transition intensities of this conformer are roughly the same intensity as the isotopically substituted species of the cis-trans conformer. Additionally, in a room temperature sample as was used for the two previous studies, thermally populated vibrationally excited states of the more stable conformers will have transition intensities equal to or exceeding that of the trans-gauche conformer.

To date, a satisfactory fit (with the residual error in the transition frequencies close to the experimental uncertainty) of all the transitions observed in this study has not been obtained. The basic form of the Hamiltonian, similar to that for the $(\mathrm{HCOOH})_{3^{-}}$ $\mathrm{H}_{2} \mathrm{O}$ complex, is

$$
\left.\hat{H}=\begin{array}{c}
\sigma \\
\left\langle 0^{+}\right|
\end{array} \mid \begin{array}{cc}
\hat{H}_{\text {rot }} & \left|0^{-}\right\rangle \\
\hat{H}_{t r} & \hat{H}_{\text {rot }-}+\Delta E
\end{array}\right)
$$

where $H_{t r}$ consists of Coriolis-like coupling terms that are due to the tunneling motion. It should be noted that the difference between the $0_{00}$ levels of the two tunneling states, $\Delta E$, cannot be measured directly, because a transition loop between $0_{00}\left(0^{+}\right)$and $0_{00}\left(0^{-}\right)$cannot 

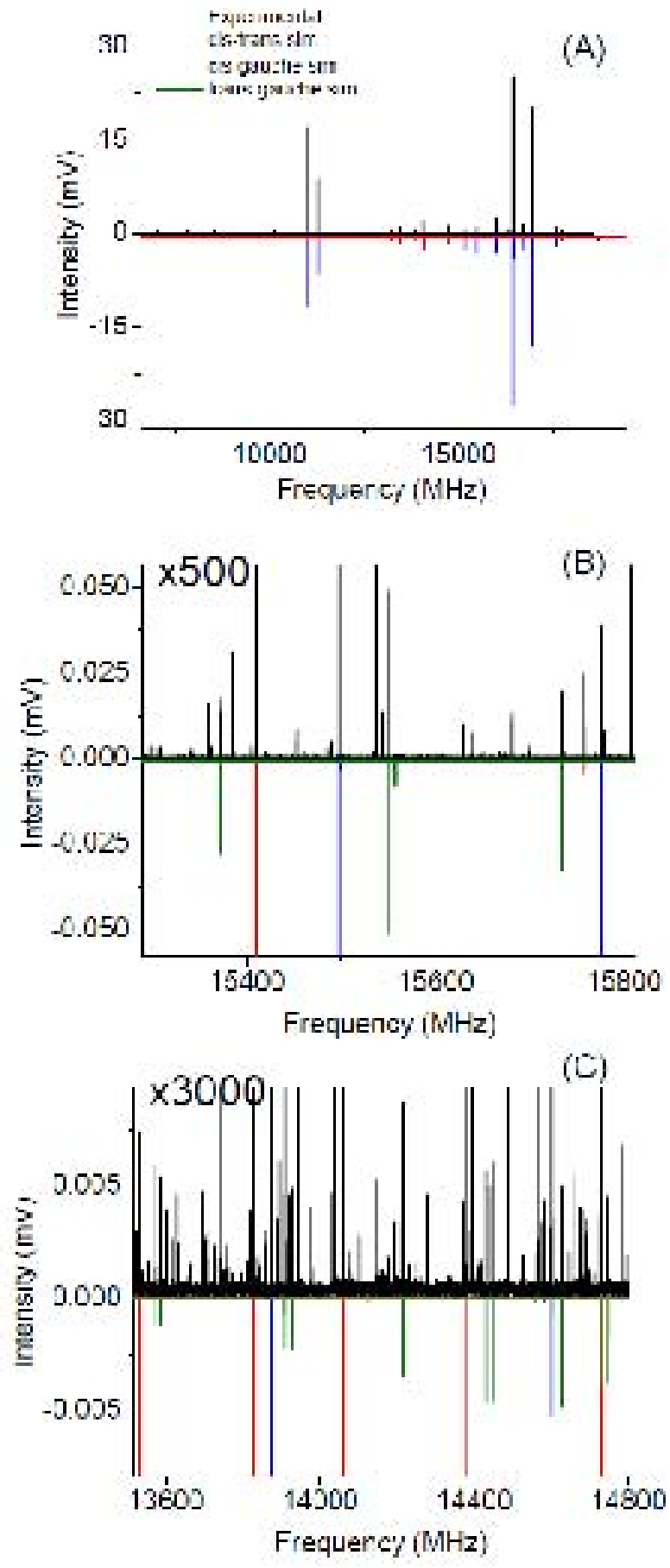

Figure 5.6. CP-FTMW spectra of ethyl formate with the application of a discharge nozzle. Panel (A) shows the spectrum on full scale, dominated by the cis-trans and cisgauche conformers, panel (B) shows the trans-gauche $J=3-2 a$-type transitions, and panel (C) shows part of the $c$-type $K_{a}=1 \leftarrow 0 Q$-branch. 
be established. This is due to the absence of measured $b$-type transitions; the calculated $b$-type dipole moment component is very small, but nonzero $(0.08 \mathrm{D})$; if $b$-type transitions could be measured, most likely using microwave-microwave double resonance measurements, this could establish definitively the value of $\Delta E$. The gap between corresponding $c$-type transitions $\left(0^{+} \leftarrow 0^{-}\right.$and $\left.0^{-} \leftarrow 0^{+}\right)$is roughly $20 \mathrm{MHz}$. If the $A, B$, and $C$ rotational constants were the same between the two states, this would mean that the value of $\Delta E$ is $\sim 10 \mathrm{MHz}$ (half of the splitting of corresponding transitions crossing the gap). However, a difference in the $A$ rotational constant between the two tunneling species would also be exhibited as a shift in the gap between $c$-type transitions, and therefore the exact value of $\Delta E$ is not known. The absence of observed $b$-type transitions separates transitions into two unconnected sets, across which no observed transition frequencies cross. This concept is illustrated in Figure 5.7.

A fit to the transitions involving $K_{a}=0$ and 1 states has been performed which fits all 63 transitions between these states to experimental uncertainty. The Hamiltonian parameters of this fit, compared to calculated parameters, are presented in Table 5.7. The $H_{t r}$ component consists of a single term, called $G_{\mathrm{a}}$, for which the operator is $P_{\mathrm{a}}$. Therefore (using the prolate basis, so that $a=z, b=x, c=y$ ), the Hamiltonian for $J=1$, for example, is

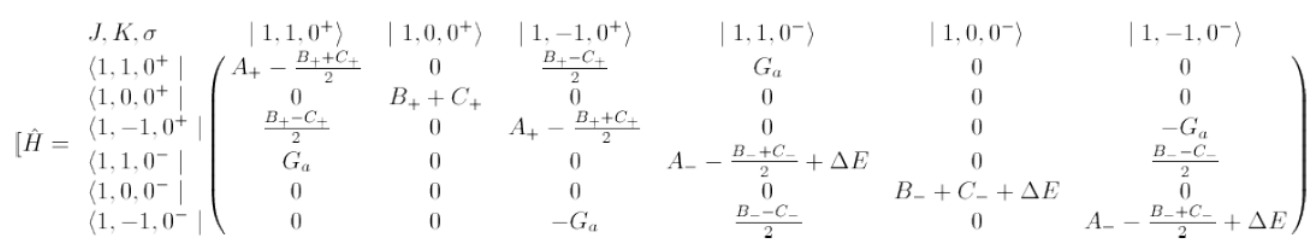




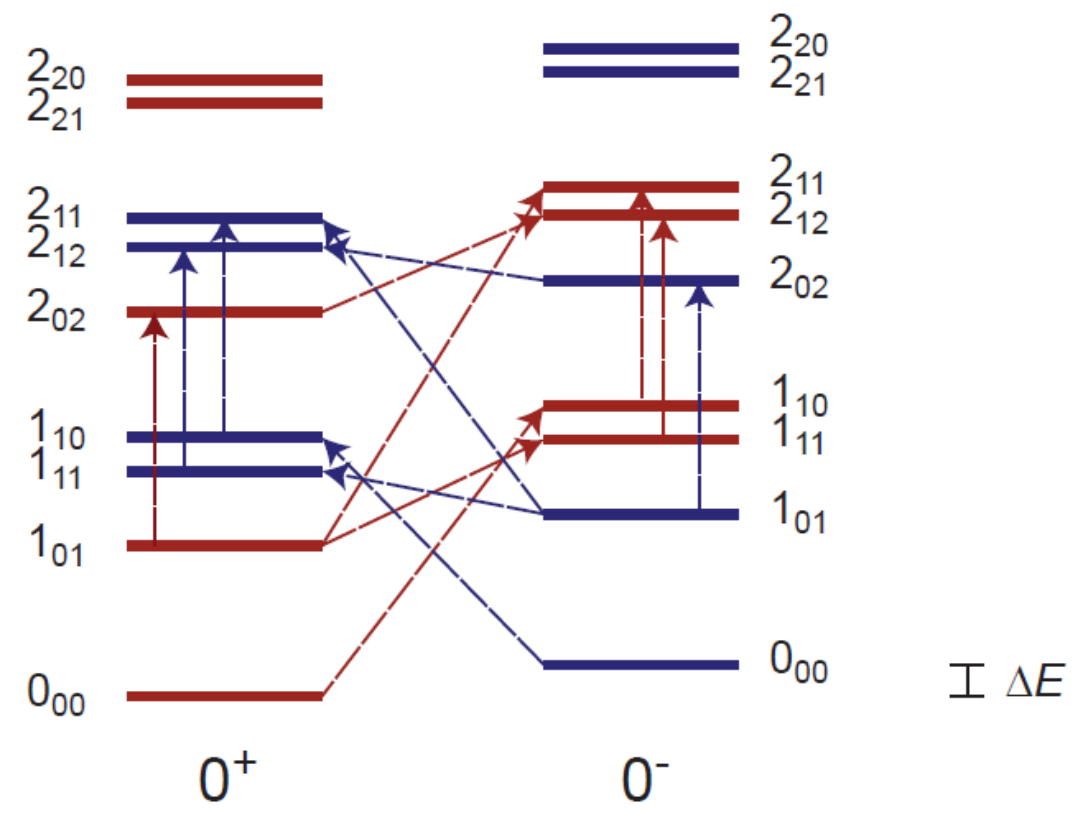

Figure 5.7. Transitions between low-J transitions of trans-gauche ethyl formate. In the absence of $b$-type transitions, the red and green colors indicate transitions between which the energy spacings can be experimentally observed. (That is, only red-to-red and greento-green transitions are allowed). The arrows indicate transitions that have been observed in the laboratory. The energies of the levels in this figure are not to scale. 
Table 5.7. Hamiltonian parameters derived from an effective fit to $K_{a}=0$ and 1 transitions of trans-gauche ethyl formate, compared to parameters calculated by electronic structure theory.

\begin{tabular}{|c|c|c|c|}
\hline \multicolumn{4}{|c|}{ Experiment } \\
\hline Parameter & $0^{+}$state & $0^{-}$state & $A b$ initio $^{\mathrm{a}}$ \\
\hline$A(\mathrm{MHz})$ & $17402.39(24)$ & $17379.59(24)$ & 16342.16 \\
\hline$B(\mathrm{MHz})$ & $2652.67795(19)$ & 2652.68514(19) & 2721.03 \\
\hline$C(\mathrm{MHz})$ & 2531.99121(19) & 2532.01952(19) & 2567.39 \\
\hline$\Delta_{\mathrm{J}}(\mathrm{kHz})$ & $3.6596(15)$ & $3.6433(15)$ & 3.58 \\
\hline$\Delta_{\mathrm{JK}}(\mathrm{kHz})$ & $-112.29(9)$ & $-94.25(9)$ & -79.26 \\
\hline$\delta_{\mathrm{J}}(\mathrm{kHz})$ & $1.0044(13)$ & $1.0096(13)$ & 1.01 \\
\hline$G_{a}(\mathrm{MHz})$ & \multicolumn{2}{|c|}{$10.219(6)$} & \\
\hline$\Delta E(\mathrm{MHz})$ & \multicolumn{2}{|c|}{$21.03(24)$} & \\
\hline$N_{\text {lines }}$ & \multicolumn{2}{|c|}{54} & \\
\hline r.m.s. error $(\mathrm{kHz})$ & \multicolumn{2}{|c|}{1.9} & \\
\hline
\end{tabular}

${ }^{\mathrm{a}}$ Calculated at an MP2/6-311++G(d,p) level of theory. 
The $G_{a}$ term has the effect of lowering the energy of the lower-energy $K_{a}=1$ state, where $K_{a}+K_{c}=J+1$ (i.e., $1_{11}$ ), and raising the energy of the higher-energy $K_{a}=1$ state. This is true regardless of the sign of $G_{a}$ (in fact, no energy levels are affected by the sign of $G_{a}$ ). The effect of this $G_{a}$ term on the energy levels decreases as a function of $J$. Finally, it should be noted that the fit value of $\Delta E$ from the fit presented in Table 5.7 is considerably higher than the value of $\sim 10 \mathrm{MHz}$ that would be expected with identical rotational constants between the two states, and the fit derives a value for $\Delta A=A_{+}-A_{-}=$ 22.8 MHz. Due to the extremely small values for $\Delta B$ and $\Delta C$, this large of a difference between the $A$ constants of the two states seems unlikely. However, there is an extremely high correlation (>0.999) between $\Delta A$ and the energy gap between the two states in the current data set.

The Hamiltonian model presented in Table 5.7, however, does an extremely poor job in fitting the $K_{a} \geq 2$ transitions measured in this study. In particular, the $G_{a}$ term has the same effect on the $K_{a} \geq 2$ energy levels as it does on the $K_{a}=1$ levels, but the effect is magnified because the matrix element $\left\langle J, K, 0^{+}|H| J, K, 0^{-}\right\rangle=K G_{\mathrm{a}}$, and therefore its magnitude increases as $K$ increases. Because of this, these transitions are split in a way that cannot be reconciled by further fitting of the parameters listed above. In Figure 5.8 this is demonstrated, comparing a simulation using the $K_{a}=0,1$ fit from Table 5.7 to the experimental spectrum in the range of higher- $K_{a}$ transitions. It should be noted that the errors in these transitions are systematic and symmetric; for example, in the $K_{a}=2$ transitions, the differences between observed and calculated frequencies between 


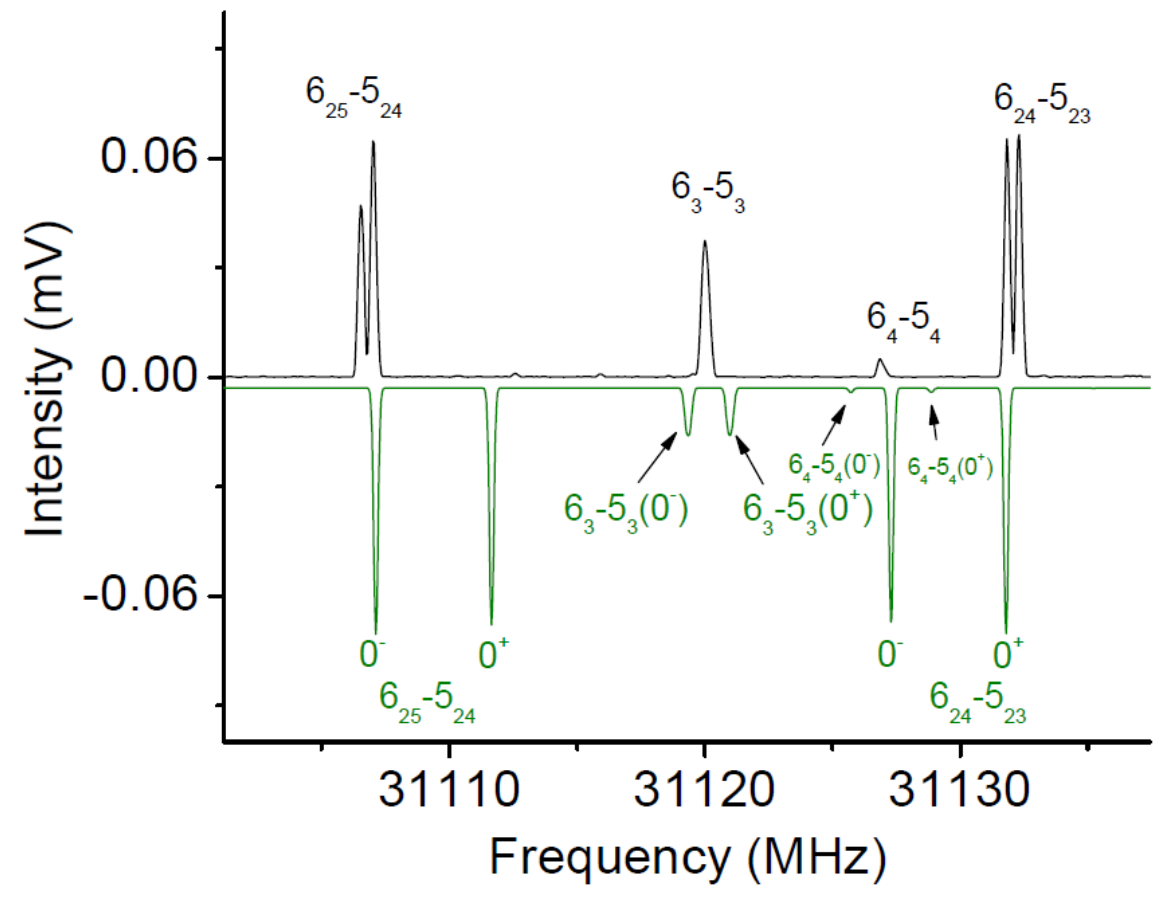

Figure 5.8. CP-FTMW spectrum of ethyl formate (black) compared to a simulation of the $J=6-5, K_{a}=2,3,4 a$-type transitions based on the effective fit presented in Table 5.7. 
corresponding transitions are equal in magnitude and opposite in sign. In fact, the average of the four $K_{a}=2$ transitions for each of the three $J$ s observed is extremely close between theory and experiment.

With all measured transitions included in the fit, the best fit to date is a two-state fit without any terms in $H_{t r}$. In this case, $\Delta A$ and $\Delta E$ are almost perfectly correlated, so $A_{+}$and $A_{-}$are constrained to be equal in this fit. The Hamiltonian parameters derived from this fit are presented in Table 5.8. Due to the considerable errors introduced to the $K_{a} \geq 2$ transitions by the $G_{a}$ term in the low- $K$ fit, this fit is expected to be more accurate in guiding searches for higher- $K$ transitions. In order to complete the characterization of the spectroscopy of this species at microwave frequencies, the transitions that are most likely to help are the $K_{a}=2 \leftarrow 1 Q$ branch near $44 \mathrm{GHz}$; these transitions are calculated to be fairly strong. This could prove useful because it would provide information on the energy spacings between the $K_{a}=1$ and 2 energy levels, which currently are not connected by any experimental information. This would help to elucidate the contributions of different Hamiltonian terms in explaining the observed spectrum. Currently work on a 40-60 GHz pulsed-jet spectrometer is underway that could measure these transitions. Alternatively, the frequencies of these transitions can be measured through microwave-microwave double resonance. The best-guess calculated frequencies of these transitions, predicted using the Hamiltonian model presented in Table 5.8, are presented in Table 5.9. The uncertainty on these transitions is estimated to be at most a few MHz, assuming that the basic model is correct. Secondly, the measurement of $b$-type 
Table 5.8. Hamiltonian parameters from a fit to all observed transitions of trans-gauche ethyl formate.

\begin{tabular}{ccc}
\hline & \multicolumn{3}{c}{ Experiment } \\
Parameter & $0^{+}$state & $0^{-}$state \\
\hline$A(\mathrm{MHz})$ & \multicolumn{2}{c}{$17391.020(55)$} \\
$B(\mathrm{MHz})$ & $2652.714(4)$ & $2652.599(4)$ \\
$C(\mathrm{MHz})$ & $2531.985(4)$ & $2532.015(4)$ \\
$\Delta_{\mathrm{J}}(\mathrm{kHz})$ & $3.71(6)$ & $3.76(6)$ \\
$\Delta_{\mathrm{JK}}(\mathrm{kHz})$ & $-101.7(7)$ & $-100.8(7)$ \\
$\delta_{\mathrm{J}}(\mathrm{kHz})$ & $1.15(9)$ & $1.18(8)$ \\
$\Delta E(\mathrm{MHz})$ & & $9.67(7)$ \\
$N_{\text {lines }}$ & & 70 \\
r.m.s. error $(\mathrm{kHz})$ & \multicolumn{3}{c}{242.6} \\
\hline
\end{tabular}


Table 5.9. Predicted frequencies of the $K_{a}=2 \leftarrow 1 Q$ branch, using the Hamiltonian model from Table 5.8 .

\begin{tabular}{cccccccc}
\hline $\mathrm{J}^{\prime}$ & $\mathrm{K}_{\mathrm{a}}{ }^{\prime}$ & $\mathrm{K}_{\mathrm{c}}{ }^{\prime}$ & $\mathrm{J}^{\prime \prime}$ & $\mathrm{K}_{\mathrm{a}}{ }^{\prime \prime}$ & $\mathrm{K}_{\mathrm{c}}{ }^{\prime \prime}$ & $\begin{array}{c}v\left(0^{+} \leftarrow 0^{-}\right) \\
(\mathrm{MHz})\end{array}$ & $\begin{array}{c}v\left(0^{-} \leftarrow 0^{+}\right) \\
(\mathrm{MHz})\end{array}$ \\
\hline 7 & 2 & 6 & 7 & 1 & 6 & 42727.284 & 42745.945 \\
6 & 2 & 5 & 6 & 1 & 5 & 43139.637 & 43158.546 \\
5 & 2 & 4 & 5 & 1 & 4 & 43494.344 & 43513.419 \\
4 & 2 & 3 & 4 & 1 & 3 & 43790.809 & 43809.989 \\
3 & 2 & 2 & 3 & 1 & 2 & 44028.546 & 44047.788 \\
2 & 2 & 1 & 2 & 1 & 1 & 44207.176 & 44226.452 \\
2 & 2 & 0 & 2 & 1 & 2 & 44569.886 & 44589.293 \\
3 & 2 & 1 & 3 & 1 & 3 & 44755.999 & 44775.509 \\
4 & 2 & 2 & 4 & 1 & 4 & 45007.732 & 45027.372 \\
5 & 2 & 3 & 5 & 1 & 5 & 45328.134 & 45347.921 \\
6 & 2 & 4 & 6 & 1 & 6 & 45720.979 & 45740.922 \\
7 & 2 & 5 & 7 & 1 & 7 & 46190.740 & 46210.837 \\
\hline
\end{tabular}


transitions, which will likely only be possible through double resonance techniques, could help to provide a firmer determination of the energy difference between tunneling substates.

\section{Interstellar Detection of trans-Methyl Formate}

a) Column density and temperature determination

The detection of trans-methyl formate in Sagittarius B2(N) was made using the PRebiotic Interstellar MOlecular Survey (PRIMOS) ${ }^{20}$ using the National Radio Astronomy Observatory 100 m Green Bank Telescope. ${ }^{21}$ Using this survey data, seven transitions of trans-methyl formate have been confidently determined in this source, all in absorption against the microwave background. The continuum toward Sgr B2(N) has been observed to be consistent with nonthermal synchrotron emission. ${ }^{22}$ The GBT observations are summarized in Table 5.10, where the line intensities and widths have been fit to Gaussian profiles. The most convincing evidence for this detection is shown in Figure 5.9, where detections of the $J=1-0,2-1$, and 3-2, $K_{a}$ transitions of the $A$ and $E$ symmetry species are presented. The $A-E$ splitting varies significantly between these three pairs of transitions (83.21 MHz, 120.81 MHz, and 73.94 MHz for $J=1-0,2-1$, and 3-2, respectively), so the identification of each of these transition pairs with equal intensities, local standard of rest (LSR) velocities, and linewidths serve as conclusive evidence for the presence of trans-methyl formate in Sgr B2(N). A fourth transition of the $A$ symmetry species, the $J_{K a K c}=21_{11} 1_{10}$ transition, has also been confidently detected; 
Table 5.10. Transitions of trans-methyl formate confidently detected toward Sgr B2(N). ${ }^{\text {a }}$

\begin{tabular}{|c|c|c|c|c|c|c|c|c|c|}
\hline \multicolumn{10}{|l|}{$A$ species } \\
\hline Transition & $\begin{array}{c}\text { Frequency } \\
(\mathrm{MHz})\end{array}$ & $\begin{array}{c}S \mu^{2} \\
\left(\text { debye }^{2}\right)\end{array}$ & $\begin{array}{c}E_{l} \\
(\mathrm{~K})\end{array}$ & $\begin{array}{c}\theta_{\mathrm{HPBW}} \\
(\operatorname{arcsec})\end{array}$ & $\begin{array}{c}T_{\mathrm{c}} \\
(\mathrm{K})\end{array}$ & $\eta_{\mathrm{B}}$ & $\begin{array}{c}V_{L S R} \\
\left(\mathrm{~km} \mathrm{~s}^{-1}\right)\end{array}$ & $\begin{array}{l}\Delta T_{a}^{*} \\
(\mathrm{mK})\end{array}$ & $\begin{array}{c}\Delta V \\
\left(\mathrm{~km} \mathrm{~s}^{-1}\right)\end{array}$ \\
\hline $1_{01}-0_{00}$ & 9124.221 & 17.07 & 0 & 81 & 25.6 & 0.8616 & $60.7(6)$ & $-11.8(7)$ & $12.4(12)$ \\
\hline $2_{02}-1_{01}$ & 18247.038 & 34.15 & 0.438 & 41 & 10.66 & 0.7423 & $64.5(4)$ & $-20.9(12)$ & $13.3(9)$ \\
\hline $2_{11}-1_{10}$ & 18575.916 & 25.61 & 3.248 & 40 & 10.88 & 0.7383 & $67.8(6)$ & $-11.7(7)$ & $19.0(12)$ \\
\hline $3_{03}-2_{02}$ & 27367.048 & 51.22 & 1.315 & 27 & 7.11 & 0.6396 & $64.9(3)$ & $-21.2(16)$ & $9.4(8)$ \\
\hline \multicolumn{10}{|l|}{$E$ species } \\
\hline Transition & $\begin{array}{c}\text { Frequency } \\
(\mathrm{MHz})\end{array}$ & $\begin{array}{c}S \mu^{2} \\
\left(\text { debye }^{2}\right)\end{array}$ & $\begin{array}{c}E_{l} \\
(\mathrm{~K})^{\mathrm{b}}\end{array}$ & $\begin{array}{c}\theta_{\mathrm{HPBW}} \\
(\operatorname{arcsec})\end{array}$ & $\begin{array}{l}T_{\mathrm{c}} \\
(\mathrm{K}) \\
\end{array}$ & $\eta_{\mathrm{B}}$ & $\begin{array}{c}V_{L S R} \\
\left(\mathrm{~km} \mathrm{~s}^{-1}\right)\end{array}$ & $\begin{array}{l}\Delta T_{a}^{*} \\
(\mathrm{mK})\end{array}$ & $\begin{array}{c}\Delta V \\
\left(\mathrm{~km} \mathrm{~s}^{-1}\right)\end{array}$ \\
\hline $1_{0}-0_{0}$ & 9207.427 & 18.03 & 2.066 & 81 & 25.6 & 0.8604 & $64.0(7)$ & $-9.4(6)$ & $18.1(14)$ \\
\hline $2_{0}-1_{0}$ & 18367.848 & 35.65 & 2.508 & 40 & 10.66 & 0.7408 & $62.9(12)$ & $-19.2(7)$ & $7.6(12)$ \\
\hline $3_{0}-2_{0}$ & 27440.992 & 52.56 & 3.390 & 27 & 7.11 & 0.6388 & $70.3(5)$ & $-22.7(10)$ & $24.3(14)$ \\
\hline
\end{tabular}

${ }^{\mathrm{a}}$ Column definitions: $S \mu^{2}$, the product of the intrinsic line strength of the transition and the square of the $a$-type dipole moment; $E_{l}$, lower-state energy; $\theta_{\mathrm{HPBW}}$, half-power beam width; $T_{\mathrm{c}}$, source continuum temperature; $\eta_{\mathrm{B}}$, telescope efficiency (assuming a surface error of $390 \mu \mathrm{m}) ; V_{\mathrm{LSR}}$, local-standard-of-rest velocity of the transition; $\Delta T_{a}{ }^{*}$, change in antenna brightness temperature due to the transition; $\Delta V$, velocity width (FWHM).

${ }^{\mathrm{b}}$ Relative to the lowest-energy $E$ species rotational level; due to internal rotor effects, this is the $J=1, K=1$ level. 


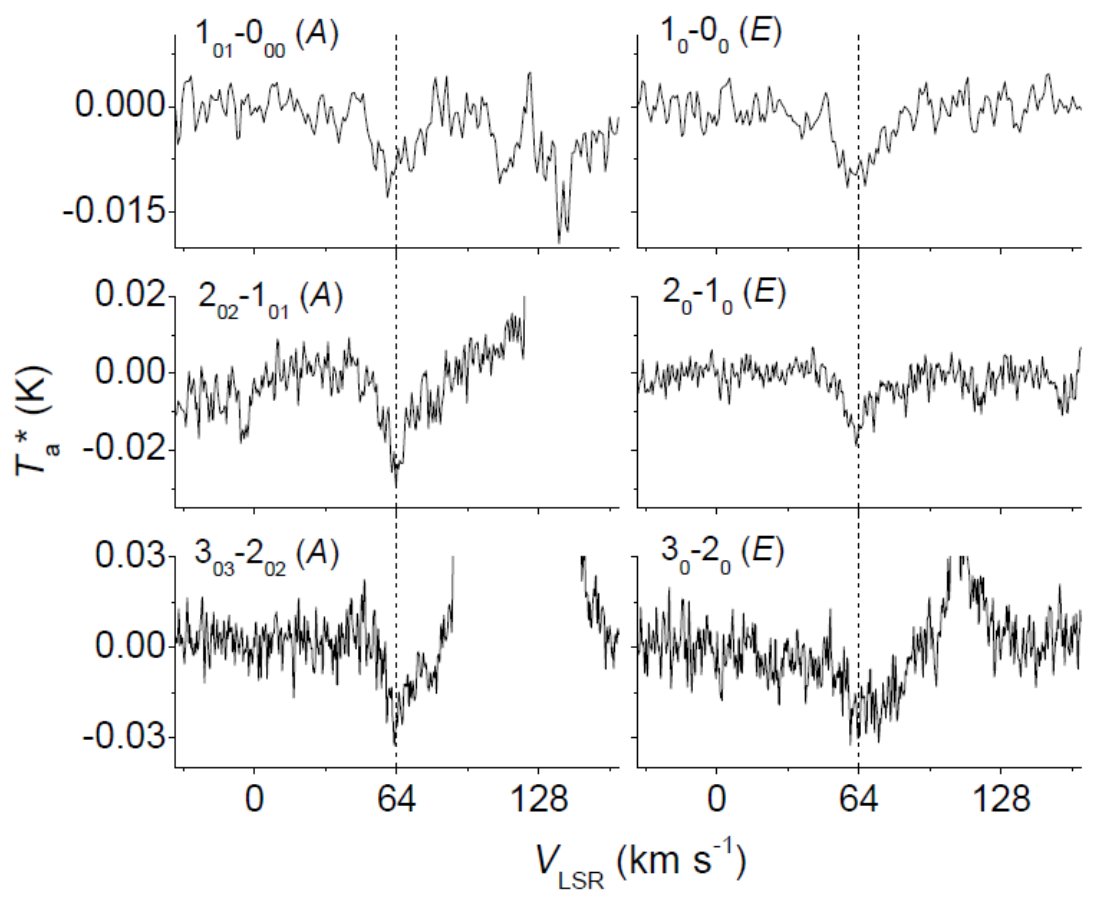

Figure 5.9. Plot of $K_{a}=0$ transitions of trans-methyl formate observed in Sgr B2(N). The strong emission features seen near the $2_{01}-1_{01}(A), 3_{03}-2_{02}(A)$, and $3_{0}-2_{0}(E)$ transitions are due to vibrationally excited states of $\mathrm{HC}_{3} \mathrm{~N}$. 
due to the large energy level shifts in the $E$ species due to the low-barrier methyl internal rotation, the corresponding transition in the $E$ species has a much higher lower-state energy $\left(E_{l}=10.03 \mathrm{~K}\right)$, and so this transition could not be measured under the supersonic expansion conditions employed in the laboratory.

In order to estimate the excitation temperature of trans-methyl formate in this rotation, we compare the intensities of the $2_{02}-1_{01}$ and $2_{11}-1_{10}$ transitions of the $A$ species. Because these two transitions are close in frequency, the GBT beamwidth is nearly the same for the two transitions and so samples the same spatial region. However, since the difference in the lower-state energies of the two transitions is reasonably large, the intensity ratio between them depends strongly on excitation temperature. The GBT observations of these two transitions are shown in panel (A) of Figure 5.10, while the calculated intensity ratio between the two transitions as a function of excitation temperature is shown in panel (B). Based on the observed relative intensity ratio of 0.56 \pm 0.05 , a rotational temperature of $7.6 \pm 1.5 \mathrm{~K}$ for trans-methyl formate in $\mathrm{Sgr} \mathrm{B} 2(\mathrm{~N})$ is derived.

Using this temperature, a beam-averaged column density for trans-methyl formate was derived, using the equation for absorption features, ${ }^{23}$

$$
N_{T}=8.5 \times 10^{9} \times \frac{Q_{r}\left(\Delta T_{a}^{*} \Delta V / \eta_{B}\right)}{\left(T_{e x}-T_{c} / \eta_{B}\right) S \mu^{2}\left(e^{-\frac{E_{l}}{T_{e x}}}-e^{-\frac{E_{u}}{T_{e x}}}\right)}
$$

where $Q_{r}$ is the rotational partition function; $\eta_{B}$ is the telescope beam efficiency; $T_{\mathrm{ex}}$ is the excitation temperature; $T_{c}$ is the source continuum temperature; $S \mu^{2}$ is the product 

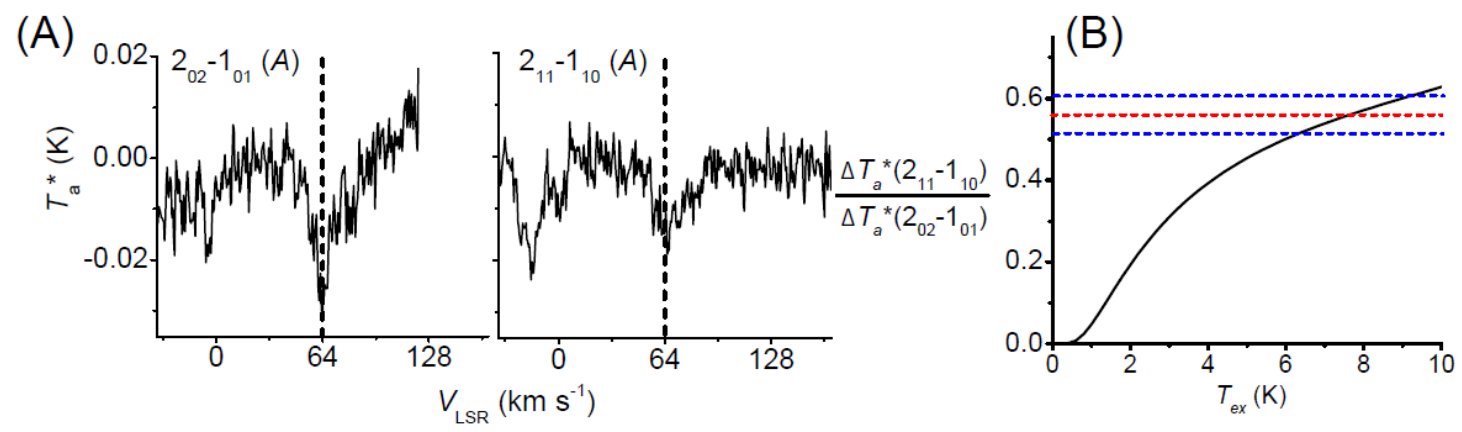

Figure 5.10. Temperature determination of trans-methyl formate in Sgr B2(N). Panel A: The $22_{02}-1_{01}$ and $22_{11}-1_{10} A$ species transitions. Panel B: Relative intensity of these two transitions as a function of excitation temperature. The red horizontal line indicates the value observed in Sgr B2(N), with the blue lines indicates the error bars. 
of the transition line strength and the square of the dipole moment in debye ${ }^{2}$; and $E_{l}$ and $E_{u}$ are the energies of the lower and upper rotational levels. Due to the dramatically different energy level structures between the $A$ and $E$ species, we determined column densities for the two torsional subspecies separately. For the $A$ species, we used a value of $Q_{r}=4.67 T_{\mathrm{ex}}{ }^{1.5}$, derived from an effective rigid-rotor fit to this species, while for the $E$ species a direct state count was performed, yielding a value of $Q_{r}=108.3$ at $7.6 \mathrm{~K}$. A value of $N_{T}$ was then derived for each detected transition. From this we derive an average column density of $1.1(+1.1,-0.5) \times 10^{13} \mathrm{~cm}^{-2}$ for the $A$ species, and $1.5(+2.0,-0.8) \times$ $10^{13} \mathrm{~cm}^{-2}$ for the $E$ species, for a total column density of $2.6(+2.3,-0.9) \times 10^{13} \mathrm{~cm}^{-2}$. The column densities are the same for the $A$ and $E$ species within measurement error.

In addition to the primary LSR velocity of $+64 \mathrm{~km} / \mathrm{s}$, many molecules in Sgr B2(N) have velocity components near +73 and $/$ or $+82 \mathrm{~km} \mathrm{~s}^{-1}$, including glycolaldehyde ${ }^{24}$ and several cyanides and isocyanides. ${ }^{23}$ The signal-to-noise ratio on individual transitions of trans-methyl formate is low, so such a component for this species is not confirmed, but several of these transitions show an asymmetry in the lineshape that suggests an absorption component near these velocities. In Figure 5.11, the average of six transitions (all confidently detected transitions except the $3_{03}-2_{02}$ transition of the $A$ species, due to a strong nearby line) is presented, showing clearer evidence for these higher-velocity components.

Observations have been made of several other transitions of trans-methyl formate in Sgr B2(N). Four transitions $\left(2_{12}-1_{11}(A), 3_{13}-2_{12}(A), 2_{1}-1_{1}(E)\right.$, and $\left.3_{1}-2_{1}(E)\right)$ are not 


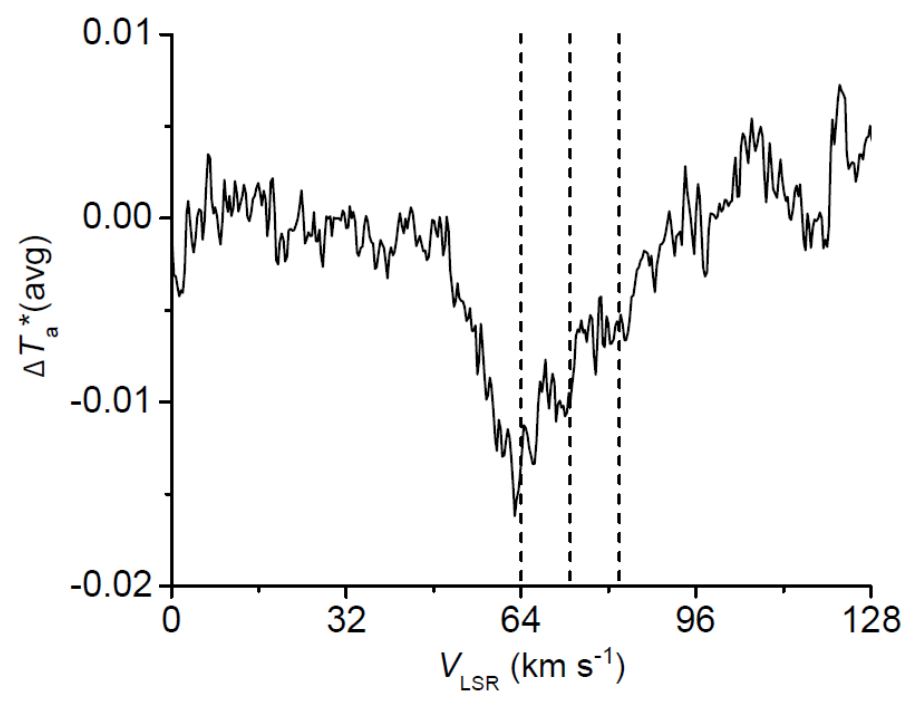

Figure 5.11. Plot of six Sgr B2 lines $\left(1_{01}-0_{00}(A), 2_{02}-1_{01}(A), 2_{11}-1_{10}(A), 1_{0}-0_{0}(E), 2_{0}-1_{0}\right.$ $(E)$, and $\left.33_{0}-2_{0}(E)\right)$ added together. The dashed lines indicate the $+64,+73$, and $+82 \mathrm{~km} / \mathrm{s}$ velocity components. 
confidently detected, but the noise levels in the observations at these frequencies are comparable to the predicted intensities expected based on the temperature and column density parameters derived above, and tentative evidence for absorption features can be seen. GBT observations of these four transitions are shown in Figure 5.12. The $5_{05}-4_{04}$ (A) transition, which was not directly measured in the laboratory but is calculated to have a frequency of $45593.037(3) \mathrm{MHz}$, was searched for, with no detection at an $8 \mathrm{mK}$ rootmean-square noise level. As the calculated $\Delta T_{\mathrm{a}}{ }^{*}$ of this transition based on the temperature and column density listed above, using the equation for emission features from Remijan et al., ${ }^{23}$ is $30(+25,-14) \mathrm{mK}$, this suggests that the telescope beam, which has a half-power beamwidth of $16^{\prime \prime}$ at this frequency, might be sampling a different temperature and/or density of trans-methyl formate gas than at lower frequencies.

Measurements of trans-methyl formate at higher frequencies could help to improve the determination of the abundance and temperature of trans-methyl formate in Sagittarius B2(N), as well as to probe warmer components. These observations will first require laboratory millimeter- and submillimeter-wave spectroscopic measurements. These experiments will be difficult on a room-temperature sample, as are used for many spectrometers in this frequency range, because the equilibrium population of transmethyl formate is lower than that of several isotopes $\left({ }^{13} \mathrm{C},{ }^{18} \mathrm{O}\right)$ as well as at least 50 vibrationally excited species of cis-methyl formate, which could lead to confusion limit issues. Additionally, particularly for the $E$ species for which the energy level structure is highly perturbed, extrapolation of the measured low- $J$ transitions to millimeter-wave 


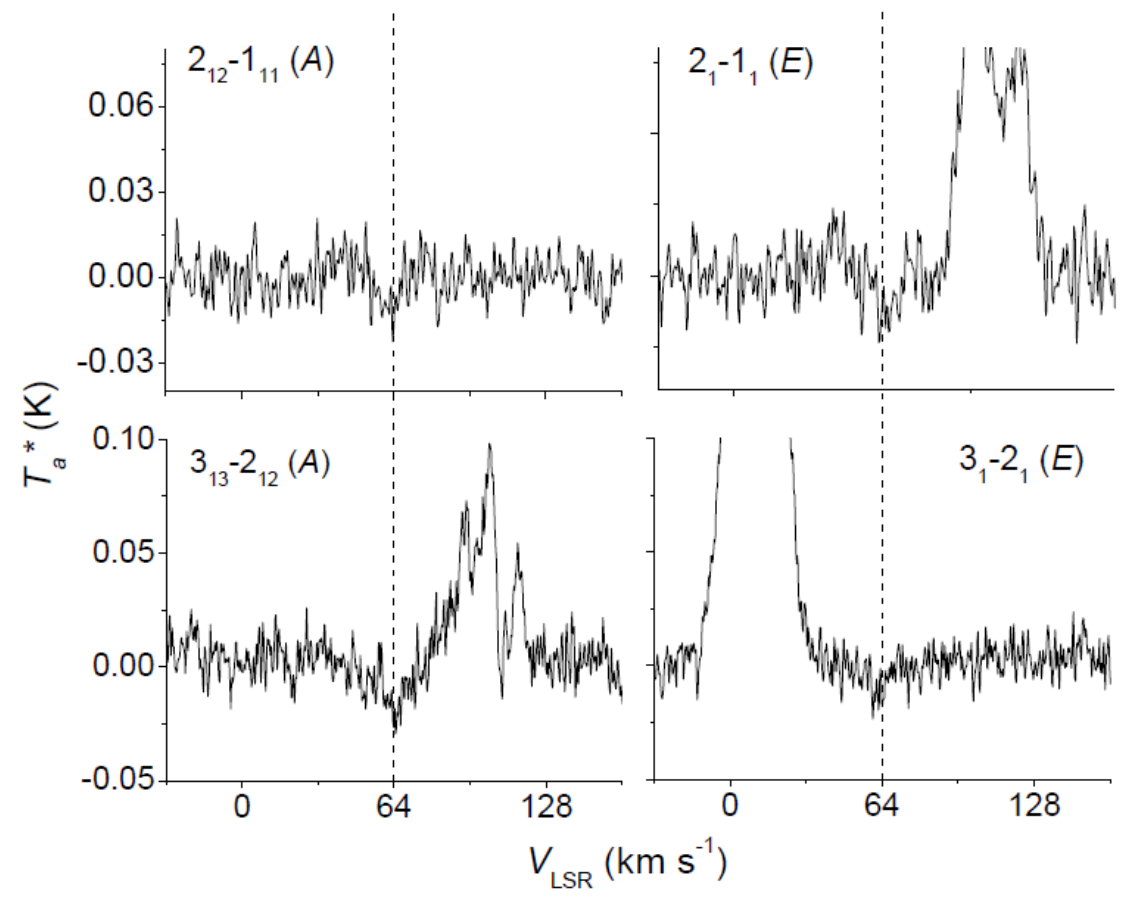

Figure 5.12. Observations of four $K_{\mathrm{a}}=1$ transitions of trans-methyl formate. 
frequencies is likely to result in significant error. An efficient discharge method for producing a large abundance of trans-methyl formate for millimeter-wave study, similar to that employed in this study at microwave frequencies, will likely be needed.

\section{b) Discussion}

The interstellar detection of trans-methyl formate represents one of the first instances where more than one conformational isomer of a molecule has been detected in the interstellar medium; this is partially due to the fact that larger molecules, which are more likely to have multiple conformers, are more difficult to observe because these molecules have larger rotational partition functions, and typically have lower abundances. Nevertheless, there are two molecules, ethanol and vinyl alcohol, for which detections of multiple conformers have been reported. Both conformations (trans and gauche) of ethanol have been detected in the Orion KL region. ${ }^{25}$ Because the energy difference between these two conformers $(57 \mathrm{~K})$ and the barrier to interconversion $(560 \mathrm{~K})$ are small, ${ }^{25}$ the higher-energy gauche conformer could be thermally excited at hot core temperatures. For vinyl alcohol $\left(\mathrm{CH}_{2} \mathrm{CHOH}\right)$, the syn and anti conformers have both been detected in Sgr B2(N). ${ }^{26}$ Due to the small number of observed lines in this study (five for the anti conformer, two for syn), the temperatures and column densities of the two conformers could not be independently determined. However, the large energy difference between these conformers $(1100 \mathrm{~K})$ suggests that an observable abundance of the higher-energy anti conformer would be due to kinetic effects. Detections of the two conformers (cis and trans) of ethanimine $\left(\mathrm{CH}_{3} \mathrm{CHNH}\right)$, using the PRIMOS survey of Sgr 
$\mathrm{B}(\mathrm{N})$, have been made in our laboratory, with what appears to be a non-equilibrium population ratio, but a careful analysis of the column densities and temperatures of these conformers is still in progress.

The observation of trans-methyl formate in Sgr B2(N) at a dramatically higher abundance than would exist under local thermodynamic equilibrium conditions strongly suggests that its formation process is conformationally selective. Moreover, all detected features of trans-methyl formate are found in absorption, while nearly all transitions of cis-methyl formate are observed in emission in Sgr B2(N) in the PRIMOS survey (up to $26 \mathrm{GHz}$ ). This suggests that the rotational temperature of trans-methyl formate is considerably lower than that of cis. Several determinations of the temperature and column density of cis-methyl formate have been performed. Cummins et al. ${ }^{27}$ derived a value of $(3.4 \pm 0.3) \times 10^{14} \mathrm{~cm}^{-2}$ and a temperature of $23 \mathrm{~K}$, and Turner $^{28}$ derived a value of $24.8 \mathrm{~K}$ and a column density of $1.1(+0.7,-0.4) \times 10^{15} \mathrm{~cm}^{-2}$. However, more recent analysis using the Turner $2 \mathrm{~mm}$ survey ${ }^{29}$ has suggested a higher temperature and column density $\left(150 \mathrm{~K}, \sim 10^{-18} \mathrm{~cm}^{-2}\right)$. The determination of a beam-averaged column density from a single-dish telescope is likely of little physical importance, since interferometric observations have shown that this conformer is predominantly found in the warm, compact $\left(\leq 5^{\prime \prime}\right)$ Large Molecule Heimat $(\mathrm{LMH})$ hot core. ${ }^{30,31}$ Alternatively, the low rotational temperature of trans-methyl formate observed in the PRIMOS survey is similar to the that of a number of other molecules, including glycolaldehyde $\left(\mathrm{CH}_{2} \mathrm{OHCHO}\right),{ }^{24}$ acetamide $\left(\mathrm{CH}_{3} \mathrm{CONH}_{2}\right),{ }^{32}$ propenal $\left(\mathrm{CH}_{2} \mathrm{CHCHO}\right),{ }^{33}$ propanal $\left(\mathrm{CH}_{3} \mathrm{CH}_{2} \mathrm{CHO}\right),{ }^{33}$ and vinyl alcohol. ${ }^{26}$ Interferometric observations of glycolaldehyde ${ }^{31}$ and acetaldehyde 
$\left(\mathrm{CH}_{3} \mathrm{CHO}\right)^{34}$ have revealed that these two molecules, in contrast to cis-methyl formate and many other complex molecules, are spread over a region that is $\geq 60^{\prime \prime}$ in extent. The presence of complex gas-phase molecules in this region has been attributed to the presence of shocks releasing material from grain mantles, inferred from the presence of a large number of masers. ${ }^{34-36}$ Due to the observation of low-temperature trans-methyl formate in Sgr B2(N), it is likely also located in this region. Therefore, we consider production mechanisms by which trans-methyl formate could be produced at a nonthermal abundance under these conditions.

One possibility is that trans-methyl formate could be formed within ice grains at low temperatures, then ejected into the gas phase at low temperatures through shocks as is proposed for other cold complex molecules observed in this region. The conformer ratio that would be produced by grain-surface reactions is not known experimentally. The trans conformer, as mentioned above, is significantly more polar than cis, which could mean that in polar environments, the trans conformer could be stabilized relative to cis. Additionally, UV radiation has been demonstrated to induce cis-trans isomerization in methyl formate in argon matrices. ${ }^{5}$ Further experiments are required to determine whether grain-surface processes could explain the observed gas-phase abundance of trans-methyl formate.

Alternatively, the observed trans-methyl formate could be the result of secondary gas-phase processing. As discussed in Chapter 4, a gas-phase methyl cation transfer reaction would be expected, based on electronic structure calculations, to lead to the synthesis of trans-methyl formate. Because the reaction to form the trans isomer does 
not have an activation barrier, it could occur at low temperatures if the reactants, formic acid and protonated methanol, are present in the gas phase. A modeling study by Laas et $a l{ }^{37}$ found that this reaction would be expected to occur efficiently when formic acid and methanol are abundant in the gas phase. In this model, these species are formed on grains at low temperatures, then thermally desorb into the gas phase at $\sim 70-100 \mathrm{~K}$, so this is the temperature at which trans-methyl formate is produced by this model. If a non-thermal desorption process, such as UV photodesorption, ${ }^{38}$ were to occur instead to release methanol and formic acid into the gas phase at lower temperatures, then methanol protonation, followed by the methyl cation transfer reaction to produce trans-methyl formate, might occur efficiently.

Another gas-phase process to produce methyl formate that could proceed at low temperatures would be a neutral-neutral radical radiative association reaction, ${ }^{39} e . g$.

$$
\mathrm{HCO}+\mathrm{OCH}_{3} \rightleftarrows\left(\mathrm{HCOOCH}_{3}\right)^{*} \rightarrow \mathrm{HCOOCH}_{3}+h v
$$

where two radicals combine to form vibrationally excited methyl formate; at the densities typical for interstellar clouds, the dominant relaxation mechanism is through emitting infrared radiation. ${ }^{40}$ This excited species, in addition to dissociating back into the reactants, could also undergo a unimolecular dissociation process, leading to $\mathrm{CH}_{3} \mathrm{OH}+\mathrm{CO}$ or $\mathrm{H}_{2} \mathrm{CO}+\mathrm{H}_{2} \mathrm{CO}$; these processes have activation barriers of about 300 $\mathrm{kJ} / \mathrm{mol},{ }^{41,42}$ considerably less than the $450 \mathrm{~kJ} / \mathrm{mol}$ released in the recombination step. ${ }^{43}$ Therefore, the excited methyl formate molecule would have to emit multiple infrared photons before the dissociation process is energetically inaccessible, which could make the product yield of reaction 5.5 very low. Additionally, because the reactants in 
Equation 5.5 are both neutral, the collision cross sections would be lower than for an ionneutral reaction, and so the overall reaction rate would be lower given equal densities. The conformational distribution that would result from this reaction is not known; however, the excited methyl formate intermediate contains far more internal energy than is required for conformational isomerization, so it would be likely to form a mix of cis and trans. Experimental and/or theoretical work will be needed to fully assess the viability of this reaction.

Formic acid has a similar conformational potential energy surface to that of methyl formate, with the methyl group of methyl formate replaced by a hydrogen atom. The barriers for conversion between conformers are likewise similar, though the lighter rotor of formic acid (predominantly an $\mathrm{H}$ atom) means that tunneling between conformers could contribute to the dynamics. To date, all interstellar detections of formic acid have been of the more energetically stable conformer (referred to in the literature as $\operatorname{trans} s^{44}$ ). The rotational spectrum of the less stable conformer of formic acid, referred to in the literature as cis, has been assigned, ${ }^{45,46}$ but to date has not been detected in the interstellar medium. Further searches for this conformer could be useful in constraining the chemistry that could lead to trans-methyl formate; the methyl cation transfer gas-phase reaction cannot generate formic acid, but a gas-phase radical-radical combination reaction or grain synthesis could be expected to produce formic acid with a similar conformational distribution to that of methyl formate. 


\section{Conclusion}

In this section the first laboratory rotational spectroscopic characterization of the higher-energy conformational isomers of methyl and ethyl formate have been reported. These studies were motivated by the multispecies chemical mapping of the Orion KL region reported in Chapter 4. Even though the existence of both of these species had been known for decades, and studied theoretically several times, their rotational spectra had not yet been measured due to the low populations of these conformers at thermal equilibrium. However, based on the prediction that trans-methyl formate could be produced efficiently by a methyl cation transfer reaction between formic acid and protonated methanol, high-sensitivity CP-FTMW spectroscopy, cavity FTMW spectroscopy, and microwave-microwave double resonance spectroscopy were employed to measure the spectra of trans-methyl formate and the corresponding isomer, transgauche, of ethyl formate. Both spectra were complicated by the existence of low-barrier tunneling motions.

Based on the transition frequencies measured in this study, trans-methyl formate has been detected for the first time in the interstellar medium, in the Sagittarius B2(N) molecular cloud, using the publicly available PRIMOS survey from the Green Bank Telescope. This detection represents the clearest example to date of conformationally selective kinetics in the ISM. Several processes that could lead to this highly nonequilibrium abundance of trans-methyl formate have been discussed. While the methyl cation transfer reaction discussed in Chapter 4 is not necessarily the reaction leading to the observed abundance of trans-methyl formate in Sagittarius B2(N), this 
study nevertheless presents the utility that broadband spectral coverage in interferometric measurements and laboratory spectroscopic techniques offer in the detection of new interstellar molecules, particularly when coupled to unbiased broadband spectral surveys of regions where rich interstellar chemistry is occurring. These techniques can be used to propose and test mechanisms for molecular formation in the interstellar medium, which can provide valuable input to theoretical models of interstellar chemistry to understand the physical and chemical conditions of star-forming regions.

The techniques of chirped pulse Fourier transform spectroscopy in the microwave region, the $100 \mathrm{~m}$ single-dish GBT, and the EVLA interferometer make a trio of techniques that can operate in the microwave region $(2-40 \mathrm{GHz})$ to measure laboratory and interstellar spectra. However, for most interstellar molecules, particularly in warmer regions, their most intense features are in the millimeter-to-submillimeter region of the electromagnetic spectrum $(40 \mathrm{GHz}-3 \mathrm{THz})$. ALMA will soon revolutionize the spatial mapping in the atmospheric windows in this region, and new facilities such as the Herschel Space Observatory are already showing promising results in visualizing astronomical objects at these frequencies. Therefore, spectral techniques implementing broadband spectral coverage are of great importance in interrogating interstellar chemistry in the laboratory. Chirped-pulse millimeter wave spectroscopy, using the principles of CP-FTMW spectroscopy, has been demonstrated in the past few years and shows great promise for this purpose. In Chapter 6, techniques will be discussed that offer the promise of measuring up to $100 \mathrm{GHz}$ of spectral bandwidth, limited by only the bandwidth of the frequency multiplier chains used to create and detect the millimeter- 
wave radiation, in sub-millisecond timescales. These techniques offer great potential for the future of laboratory astrochemistry, as well as for other analytical chemistry applications of rotational spectroscopy, including trace gas monitoring, atmospheric chemistry, and chemical kinetics, fields which can benefit from the high time resolution these techniques can offer. 


\section{Chapter 5 References}

(1) Ruschin, S.; Bauer, S. H. J. Phys. Chem. 1980, 84, 3061-3065.

(2) Grindley, T. B. Tetrahedron Lett. 1982, 23, 1757-1760.

(3) Cain, D.; Pawar, D. M.; Stewart, M.; Billings, H.; Noe, E. A. J. Org. Chem. 2001, $66,6092-6095$.

(4) Blom, C. E.; Günthard, H. H. Chem. Phys. Lett. 1981, 84, 267-271.

(5) Müller, R. P.; Hollenstein, H.; Huber, J. R. J. Mol. Spectrosc. 1983, 100, 95-118.

(6) Curl, J. R. F. J. Chem. Phys. 1959, 30, 1529-1536.

(7) Senent, M. L.; Villa, M.; Melendez, F. J.; Dominguez-Gomez, R. Astrophys. J. 2005, 627, 567-576.

(8) Riveros, J. M.; Wilson, J. E. B. J. Chem. Phys. 1967, 46, 4605-4612.

(9) Suenram, R. D.; Grabow, J. U.; Zuban, A.; Leonov, I. Rev. Sci. Instrum. 1999, 70, 2127-2135.

(10) Grabow, J.-U.; Palmer, E. S.; McCarthy, M. C.; Thaddeus, P. Rev. Sci. Instrum. 2005, 76, 093106-093111.

(11) McCarthy, M. C.; Chen, W.; Travers, M. J.; Thaddeus, P. Astrophys. J. Suppl. Ser. 2000, 129, 611-623.

(12) Kleiner, I. J. Mol. Spectrosc. 2010, 260, 1-18.

(13) Hougen, J. T.; Kleiner, I.; Godefroid, M. J. Mol. Spectrosc. 1994, 163, 559-586.

(14) Suenram, R. D.; Golubiatnikov, G. Y.; Leonov, I. I.; Hougen, J. T.; Ortigoso, J.; Kleiner, I.; Fraser, G. T. J. Mol. Spectrosc. 2001, 208, 188-193.

(15) Subramanian, R.; Novick, S. E.; Bohn, R. K. J. Mol. Spectrosc. 2003, 222, 57-62. 
(16) Ruoff, R. S.; Klots, T. D.; Emilsson, T.; Gutowsky, H. S. J. Chem. Phys. 1990, $93,3142-3150$.

(17) Karakawa, Y.; Oka, K.; Odashima, H.; Takagi, K.; Tsunekawa, S. J. Mol. Spectrosc. 2001, 210, 196-212.

(18) Wiberg, K. B.; Bohn, R. K.; Jimenez-Vazquez, H. J. Mol. Struct. 1999, 485-486, 239-248.

(19) I.R. Medvedev, F. C. D. L., E. Herbst. Astrophys. J. Suppl. Ser. 2009, 181, 433.

(20) Hollis, J. M.; Remijan, A. J.; Jewell, P. R.; Lovas, F. J. The GBT PRIMOS Project, http://www.cv.nrao.edu/ aremijan/PRIMOS/.

(21) The National Radio Astronomy Observatory is a facility of the National Science Foundation, operated under cooperative agreement by Associated Universities, Inc.

(22) Hollis, J. M.; Jewell, P. R.; Remijan, A. J.; Lovas, F. J. Astrophys. J. 2007, 660, L125.

(23) Remijan, A. J.; Hollis, J. M.; Lovas, F. J.; Plusquellic, D. F.; Jewell, P. R. Astrophys. J. 2005, 632, 333.

(24) Hollis, J. M.; Jewell, P. R.; Lovas, F. J.; Remijan, A. J. Astrophys. J. 2004, 613, L45.

(25) Pearson, J. C.; Sastry, K. V. L. N.; Herbst, E.; de Lucia, F. C. Astrophys. J. 1997, $480,420$.

(26) Turner, B. E.; Apponi, A. J. Astrophys. J. 2001, 561, L207. 
(27) Cummins, S. E.; Linke, R. A.; Thaddeus, P. Astrophys. J. Suppl. Ser. 1986, 60, 819-878.

(28) Turner, B. E. Astrophys. J. Suppl. Ser. 1991, 76, 617-686.

(29) Remijan, A.J.; Leigh, D.P.; Markwick-Kemper, A.J.; Turner, B.E, 2008. arXiV:0802.2273.

(30) Mehringer, D. M.; Snyder, L. E.; Miao, Y.; Lovas, F. J. Astrophys. J. 1997, 480, L71.

(31) Hollis, J. M.; Vogel, S. N.; Snyder, L. E.; Jewell, P. R.; Lovas, F. J. Astrophys. J. 2001, 554, L81.

(32) Hollis, J. M.; Lovas, F. J.; Remijan, A. J.; Jewell, P. R.; Ilyushin, V. V.; Kleiner, I. Astrophys. J. 2006, 643, L25.

(33) Hollis, J. M.; Jewell, P. R.; Lovas, F. J.; Remijan, A. J.; Mollendal, H. Astrophys. J. 2004, 610, L21.

(34) Chengalur, J. N.; Kanekar, N. Astron. Astrophys. 2003, 403, L43-L46.

(35) Sato, F.; Hasegawa, T.; Whiteoak, J. B.; Miyawaki, R. Astrophys. J. 2000, 535, 857.

(36) Mehringer, D. M.; Menten, K. M. Astrophys. J. 1997, 474, 346.

(37) Laas, J.; Garrod, R. T.; Herbst, E.; Widicus Weaver, S. L. Astrophys. J. 2011, 728,71 .

(38) Oberg, K. I.; Bottinelli, S.; Jorgensen, J. K.; van Dishoeck, E. F. Astrophys. J. 2010, 716, 825 .

(39) Barker, J. R. J. Phys. Chem. 1992, 96, 7361-7367. 
(40) Herbst, E.; Klemperer, W. Astrophys. J. 1973, 185, 505-533.

(41) Francisco, J. S. J. Am. Chem. Soc. 2003, 125, 10475-10480.

(42) Metcalfe, W. K.; Simmie, J. M.; Curran, H. J. J. Phys. Chem. A 2010, 114, 54785484.

(43) Glockler, G. J. Phys. Chem. 1959, 63, 828-832.

(44) The conformers of formic acid are typically described by the stereochemical relationship of the two $\mathrm{H}$ atoms across the $\mathrm{C}-\mathrm{O}$ bond, whereas conformers of methyl formate are described by the relationship of the two heavy groups (=O and $-\mathrm{CH}_{3}$ ). Therefore the nomenclature is reversed between the two molecules; cismethyl formate and trans-formic acid are structurally analogous, and vice versa. Here the literature nomenclature is maintained for both molecules, to minimize confusion.

(45) Hocking, W. H. Z. Naturforsch, A: Phys. Sci. 1976, 31a, 1113-1121.

(46) Winnewisser, M.; Winnewisser, B. P.; Stein, M.; Birk, M.; Wagner, G.; Winnewisser, G.; Yamada, K. M. T.; Belov, S. P.; Baskakov, O. I. J. Mol. Spectrosc. 2002, 216, 259-265. 


\section{Chapter 6}

\section{Techniques for High-Bandwidth $(\geq 30$ GHz $)$ Chirped-Pulse Millimeter and Submillimeter Spectroscopy}

\section{Introduction}

To this point, this thesis has been concerned with the development and application of chirped-pulse spectroscopy in the microwave $(2-40 \mathrm{GHz})$ region of the spectrum. Particularly when coupled with a supersonic expansion molecular source, spectroscopy in this frequency range is a powerful and sensitive method for chemical identification and characterization for a variety of applications. However, techniques utilizing the same concepts at higher frequency, in the millimeter (wavelength 10-1 mm, frequency 30-300 $\mathrm{GHz}$ ) and submillimeter (wavelength $<1 \mathrm{~mm}$, frequency $>300 \mathrm{GHz}$ ) regions of the spectrum, are of significant interest because of the population distribution across energy levels in molecules at higher temperatures. If one wishes to measure the rotational spectrum of a static gas cell, the sensitivity enhancement in measuring rotational spectra at higher frequencies is vast. Spectroscopy in these regions is useful for many applications; for example, studying the spectroscopy of thermally populated vibrationally excited states (which are usually cooled out in a supersonic expansion), quantitatively probing the molecular composition of a gas sample, or measuring transition frequencies for radio astronomical observations. ${ }^{1}$

In order to demonstrate the sensitivity advantage of millimeter-wave spectroscopy for room-temperature samples, simulations of the spectra of four molecules at $300 \mathrm{~K}$ are 
presented in Figure 6.1. This figure shows that at this temperature, the transition intensity at frequencies below $40 \mathrm{GHz}$ is extremely small. While some of this advantage is mitigated by the fact that higher microwave power levels are available at low frequencies than in the millimeter/submillimeter regions, nevertheless the transition intensity at higher frequencies is greater for molecular rotational spectroscopy in static samples. It follows from this that for warmer regions of interstellar chemistry (typically with temperatures of $\sim 100 \mathrm{~K}$ and above), the strongest molecular signatures are at millimeter/submillimeter frequencies; several of the newest generation of radio astronomical facilities are targeted in this frequency range for this reason. ${ }^{2}$ Therefore, spectroscopic techniques with high sensitivity and data throughput in this frequency range are needed to complement these observatories.

It should be noted that the simulations presented in Figure 6.1 neglect the contributions of vibrationally excited states, many of which can be thermally populated at high temperatures. These effects increase as molecular size increases. The vibrational partition function of methanol at $300 \mathrm{~K}$ is calculated to be 1.3 , while for methyl formate it is 3.4 (calculated at a B3LYP/6-311++G(d,p) level of theory, with the energies of vibrationally excited states calculated using the harmonic oscillator approximation). For lactide, the room-temperature vibrational partition function is calculated to be 102 , while for suprane it is 455 ; the room-temperature spectra of these molecules become vastly more complicated because of transitions due to hot bands, and the transition intensities are lower because of the populations of these hot bands. Due to the population of 


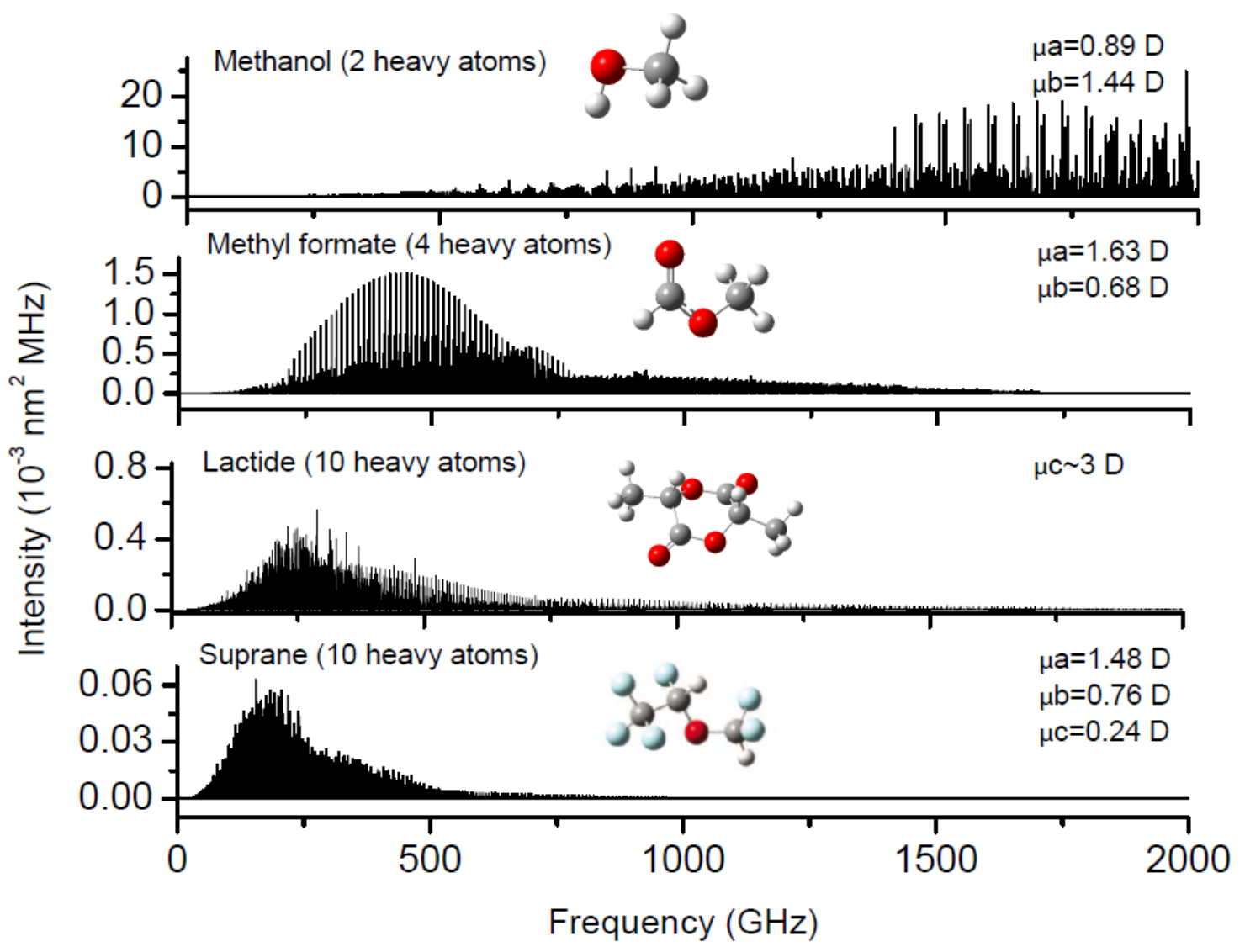

Figure 6.1. Simulated spectra of four molecules at $300 \mathrm{~K}$ : methanol $\left(\mathrm{CH}_{3} \mathrm{OH}\right)$, methyl formate $\left(\mathrm{HCOOCH}_{3}\right)$, lactide $\left(\mathrm{C}_{6} \mathrm{O}_{2} \mathrm{H}_{8}\right)$, and suprane $\left(\mathrm{C}_{3} \mathrm{~F}_{6} \mathrm{OH}_{2}\right)$. Line frequencies and intensities of methanol and methyl formate are derived from the Jet Propulsion Laboratory molecular spectroscopy catalog. ${ }^{26}$ For lactide and suprane, rotational constants determined from microwave studies were used. For lactide, $A=2202.5 \mathrm{MHz}$, $B=1197.0 \mathrm{MHz}, C=822.3 \mathrm{MHz}$; for suprane, $A=2330.14 \mathrm{MHz}, B=865.37 \mathrm{MHz}, C=$ $753.18 \mathrm{MHz}$. Contributions due to the vibrational partition function were not accounted for in the simulated transition intensity calculations. 
vibrationally excited states, additionally, structure determination of large molecules by the measurement of isotopically labeled samples at room temperature is unfeasible; for lactide approximately 1,700 vibrationally excited states are calculated to have populations that exceed that of the ${ }^{13} \mathrm{C}$ isotopoologues (1\% of the abundance of the normal species), while for suprane about 7,500 hot bands exceed this threshold.

The current spectrometers in use for measuring rotational spectra in this frequency range are relatively slow scanning instruments (compared to chirped pulse arbitrary waveform generator-driven techniques in the microwave) that measure rotational lines by absorption. The two primary sources for generating radiation in this region are solid-state active frequency multiplier chains (AMCs) and backward wave oscillators (BWOs), tube devices that are related to the TWTAs employed at microwave frequencies. Detection is either performed by bolometers or heterodyne receivers (mixers). Techniques utilizing active multiplier chains with a synthesizer as the light source are limited in scan speed by the switching time of the synthesizer, which can be on the order of milliseconds (for example, $18 \mathrm{~ms}$ in the spectrometer in use at the Jet Propulsion Laboratory). ${ }^{3}$ Several other millimeter-wave spectrometers are used in other laboratories around the world, based on similar design principles. $^{4-6}$ The FASSST spectrometer at Ohio State University ${ }^{7}$ scans significantly faster than this using a fast sweeping synthesizer, which allows for scan speeds on the order of 1-10 GHz/s. (Previous FASSST implementations have used BWOs as the frequency source. ${ }^{8,9}$ ) For a typical device bandwidth of $\sim 50 \mathrm{GHz}$ (which increases as the base frequency increases), this allows a scan of the full bandwidth of the instrument to be performed in less than a 
minute. This instrument is currently being used to explore astrophysical "weed" molecules, using the intensity dependences of molecular transitions to determine their excitation energies and allowing a forward-prediction of the entire spectrum at any arbitrary temperature. ${ }^{10}$ However, the FASSST instrument falls short of "real-time" (i.e., video frame rate, on the order of $24-60 \mathrm{~Hz}$, or $<50 \mathrm{~ms}$ measurement time) monitoring of multiple molecular concentrations. Moreover, broadband spectroscopy of transient species, such as those of astrophysical interest, or reactive intermediates, benefits from faster measurement timescales because of the short lifetimes of these species.

Two groups have pursued chirped-pulse millimeter-wave (CPmmW) spectroscopy in the past few years, with designs based on the CP-FTMW design. A conceptual schematic of this spectrometer design is presented in Figure 6.2. An AWGgenerated chirped pulse is upconverted by a phase-locked oscillator and filtered, just as in the CP-FTMW design. This pulse is then fed into an AMC, which multiplies the frequencies of the pulse (and therefore its bandwidth) by the multiplication factor of the chain. This is an important point because the AWG demands are reduced at this bandwidth compared to at microwave frequencies: for example, in the highest frequency band at which CPmmW spectroscopy has been developed $(780-870 \mathrm{GHz}$, an $\mathrm{x} 72$ multiplier chain), addressing this full $90 \mathrm{GHz}$ bandwidth requires only $1250 \mathrm{MHz}$ of AWG bandwidth. The multiplied pulse is allowed to interact with a gas sample, either in a molecular beam or a static cell. The resulting radiation (with molecular signatures imprinted) is measured by heterodyne detection, in which the local oscillator is generated 


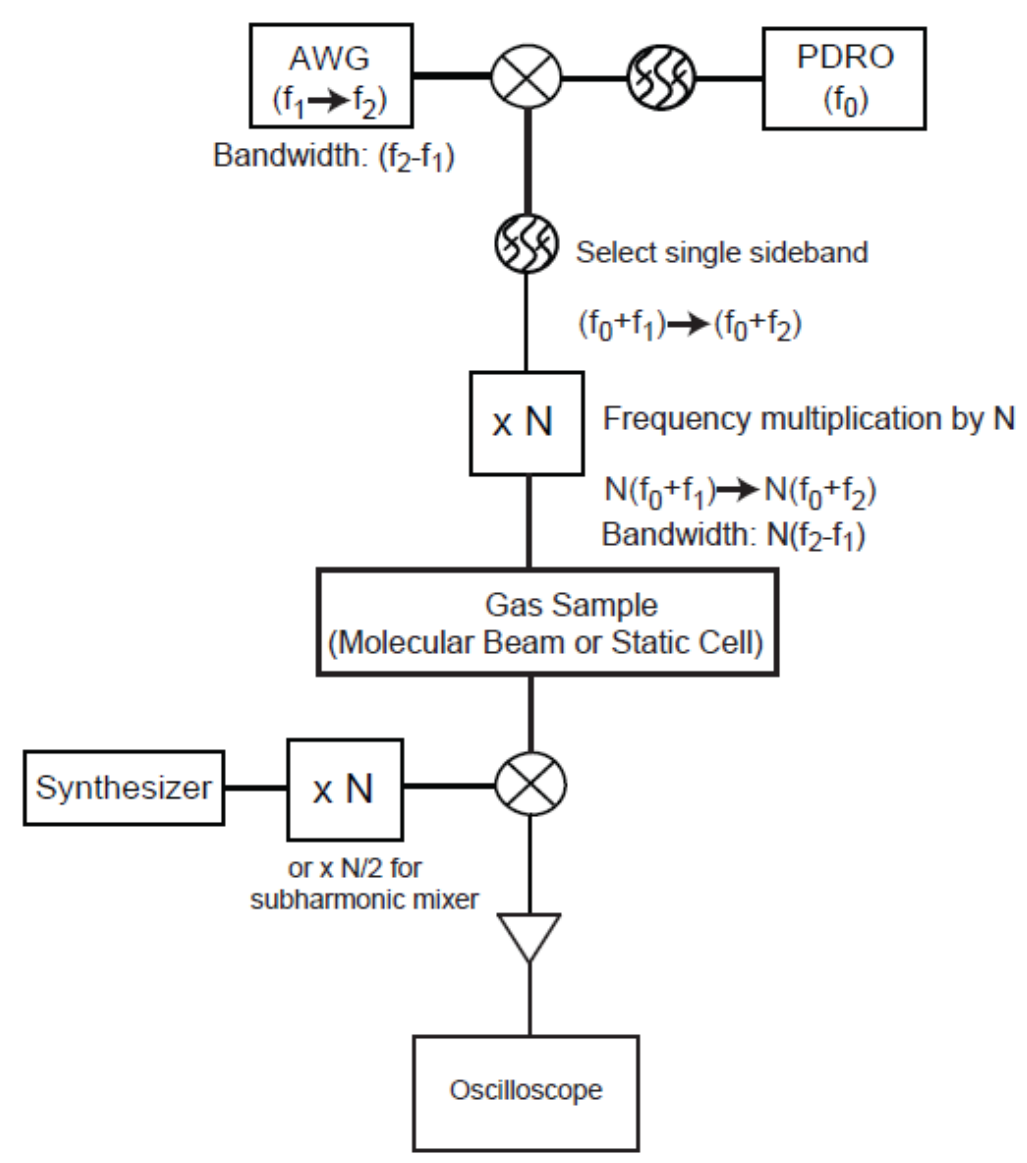

Figure 6.2. Example schematic for chirped-pulse millimeter-wave spectroscopy as implemented in previous reports, ${ }^{11,12}$ using a single-frequency local oscillator for heterodyne detection. 
by a single-frequency source (a phase-stable synthesizer or phase-locked oscillator) which passes through a second AMC (referred to as the "mix AMC"). This converts the pulse back into the microwave, where it can be amplified and digitized. This technique has been applied in the $3 \mathrm{~mm}$ range by the R.W. Field group at $\mathrm{MIT}^{11}$ and in the submillimeter in the Optical Technology Division at NIST. ${ }^{12}$

The biggest drawback of CPmmW spectroscopy as compared to CP-FTMW spectroscopy is the lower power available at this frequency ranges. Whereas amplifiers with $\sim 300 \mathrm{~W}$ of peak power was employed in the microwave studies reported in previous chapters of this thesis, currently only $\sim 30 \mathrm{~mW}$ is accessible in high-bandwidth AMCs at $\sim 100 \mathrm{GHz}$, and it falls to as low as $\sim 300 \mu \mathrm{W}$ at $\sim 840 \mathrm{GHz}$. Additionally, the high bandwidths of these devices pose a problem when using the schematic of Figure 6.2 with a single-frequency source for downconversion in terms of the digitizer demands. Currently the fastest oscilloscope available commercially is an 80 GS/s device with 32 $\mathrm{GHz}$ of hardware bandwidth, a number that is exceeded by solid-state devices in this region. This chapter describes the development of a millimeter/submillimeter chirpedpulse spectrometer that can measure both absorption and emission spectra across wide bandwidths (defined here as greater than $30 \mathrm{GHz}$ ) with a single instrument design, with low digitizer demands (ideally $500 \mathrm{MHz}$ ). This is accomplished by using the second channel of an AWG as the local oscillator rather than a single-frequency oscillator. The design principles of this spectrometer, and demonstration measurements using both absorption and emission techniques, will be presented. 


\section{Spectrometer design}

The basic schematic for high-bandwidth CPmmW spectroscopy is presented in Figure 6.3. This particular schematic was employed for spectroscopy from 780-870 GHz. A two-channel AWG of the same type employed in CP-FTMW spectroscopy was used, except with the interleave option off so that each channel has a $12 \mathrm{GS} / \mathrm{s}$ sampling rate (6 GHz of Nyquist bandwidth). The output of each channel is filtered using a $6 \mathrm{GHz}$ low-pass filter and then upconverted by a frequency-filtered $9.00 \mathrm{GHz}$ PDRO. The upper sideband from the mixing process is filtered using a bandpass filter. This filtration is crucial because of the effect that spurious outputs have in AMCs. The elimination of the other sideband (which is between 9.00 and $3.00 \mathrm{GHz}$ in this setup) is important, but equally critical is the limitation of spurious signals from the AWG. The Tektronix AWG7122B has a rated specification of $50 \mathrm{~dB}$ signal purity, which is consistent with what we have observed in the laboratory. At this intensity level, however, a large number of spurs are observed; their frequency and intensity have been seen to vary significantly with AWG frequency. While these spurs are weak enough to be insignificant for chirped-pulse spectroscopy in the microwave, when going through a multiplication circuit such as the one presented in Figure 6.3 (x72), this can cause serious problems, to the extent that without proper filtration the spurs can destroy the pulse spectral quality; however, with good filters, the pulses have good spectral purity, as will be demonstrated later. Microwave bandpass filters can be custom manufactured that have very high rejection out of band and very flat response in band. 


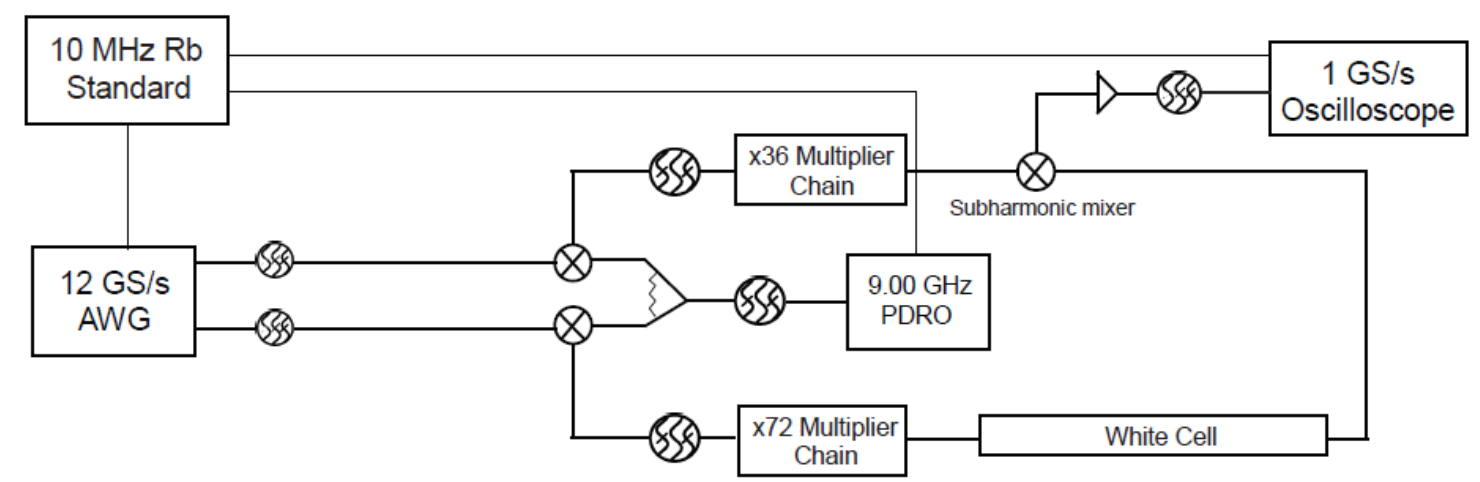

Figure 6.3. Example schematic of a chirped-pulse millimeter-wave spectrometer used in the measurements presented in this chapter, for both absorption and emission measurements. This particular implementation uses a 790-880 $\mathrm{GHz}$ active multiplier chain. Designs at $25-40 \mathrm{GHz}$ and $530-590 \mathrm{GHz}$ have also been used.i don' 
The filtered pulses are then each fed into the two multiplier chains. The chains, which for the millimeter-wave measurements presented here were manufactured by Virginia Diodes, are a series of frequency doublers and triplers with a broadcast horn antenna at the end for transmission in free space. Below $40 \mathrm{GHz}$, the chain can include amplification steps using solid-state amplifiers to increase the final power. The output of the signal AMC was broadcast into the vacuum chamber through a Teflon window. For these measurements, the gas cell was a $25 \mathrm{~m}$ pathlength White cell which has been

described in detail elsewhere. ${ }^{13,14}$ The pulse that has been transmitted through the chamber is mixed in a subharmonic mixer (that is, where the local oscillator is at half the frequency of the radiation to be downconverted) with the output of the mix AMC. The resulting radiation is amplified, filtered (both to eliminate DC components and highfrequency components), and digitized.

\section{Chirped-Pulse Frequency Combs for Absorption Spectroscopy}

Frequency combs are emerging as a powerful light source for a plethora of spectroscopic applications. ${ }^{15,16}$ In the time domain, a frequency comb is simply a repeated broad-bandwidth light pulse. When this pulse train is Fourier transformed, a spectrum emerges which consists of a broadband set of sharp lines (the "comb teeth") separated by the reciprocal of the repetition rate of the light source. Most applications so far have employed ultrafast lasers in the infrared and visible. These lasers have broad bandwidth outputs due to their short time duration. As the laser repetition rate can be controlled to extremely high precision, this results in very high precision in the 
frequencies of the comb. The coupling of this comb to a cavity is natural because the modes of the cavity are likewise linearly spaced in frequency, which provides extraordinary sensitivity enhancement.

Using chirped pulses as the light source rather than ultrashort lasers offers many of the same properties of ultrafast combs. Moreover, because the properties of the comb are determined by the arbitrary waveform generator, the user has a high degree of control over the comb. Chirped pulses likewise cover a large bandwidth, but because the pulse bandwidth is decoupled from its duration (unlike for a transform-limited pulse), the pulse can achieve a $100 \%$ duty cycle. The chirped-pulse frequency comb (CPFC) concept is demonstrated in Figure 6.4, showing the time domain and frequency domain representations. The comb structure that arises from this repeated chirped pulse can be conceptually understood in terms of constructive and destructive interference. Each frequency in the bandwidth of the chirped pulse is swept numerous times. At most frequencies, the phase of the pulse is different on each sweep, and so as the number of sweeps increases, eventually destructive interference reduces the light intensity to zero. At some frequencies, however, the phase is matched so that is the same on each sweep and so the intensity at these frequencies add constructively. The criterion for this constructive interference is that an integer number of phase cycles are executed between polarizations at this frequency; that is, that $t_{\text {rep }}=n / v$, where $t_{\text {rep }}$ is the duration of each sweep and $n$ is any integer. This leads to the result that $v_{\text {comb }}=n / t_{\text {rep. }}$. This is assuming 

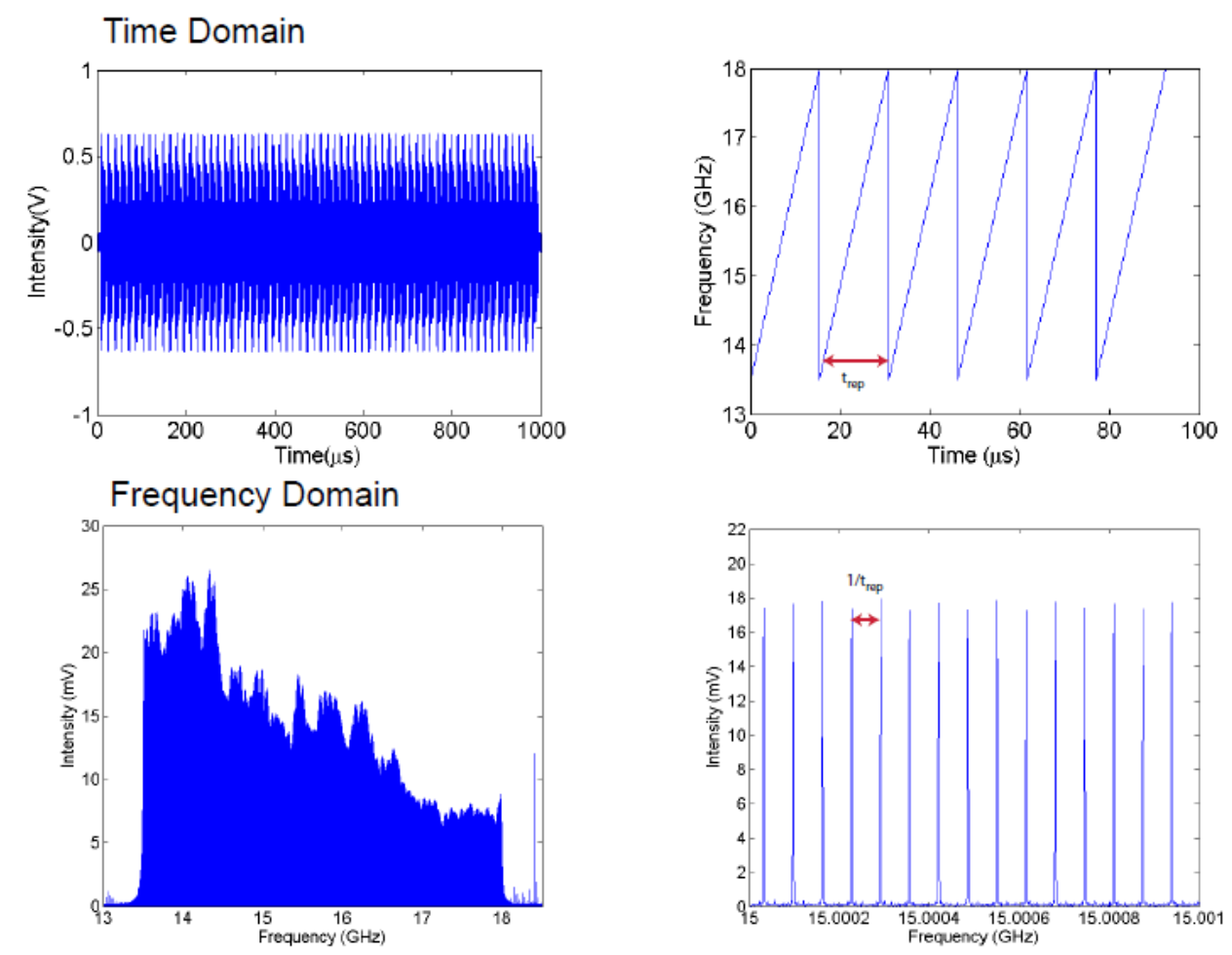

Figure 6.4. Chirped-pulse frequency combs in the microwave region. The top panels show the time domain sweep, with the pulse train in the upper left and a spectrogram in the upper right. The bottom panels show the Fourier transform of this pulse. The overall frequency profile is governed by the frequency coverage of each sweep (in this case, 13.5-18.0 GHz), while the frequency spacing between the comb teeth is the reciprocal of the duration of each sweep. 
that the phase of each chirped pulse is the same. An offset can be instituted by introducing a phase shift on each subsequent polarization. That is, the equation for the $m$ th chirped pulse of the comb is given by

$$
E_{m}(t)=E_{0} \cos \left(\omega_{\mathrm{i}}\left(t-t_{0}\right)+\frac{1}{2} \frac{\left(\omega_{\mathrm{f}}-\omega_{\mathrm{i}}\right)}{t_{\mathrm{rep}}}\left(t-t_{0}\right)^{2}+m \Delta \omega t_{\mathrm{rep}}\right)
$$

where $t_{0}$ is the start time of the $m$ th chirp. This leads to a frequency comb with tooth frequencies $v_{\text {comb }}=n / t_{\text {rep }}+\Delta \omega / 2 \pi$. This frequency offset is paralleled in cavity-enhanced laser frequency comb spectroscopy by the fact that dispersion in the cavity leads to a slight phase shift on each round-trip cycle of the comb, which has a similar effect; however, in the CPFC experiment, this frequency shift is completely user-controllable.

Digital filters of the same type used to suppress the side lobes of molecular free induction decay signals in CP-FTMW spectroscopy can be used to improve the frequency properties of a chirped-pulse frequency comb. In principle, these filters can be applied in the AWG; however, some components, particularly frequency multipliers, have limited dynamic range and so the amplitude profile is unlikely to be preserved in the comb. The frequency filters can also be applied digitally in data processing. Filters can be applied both to the individual chirped pulse sweeps and to the comb as a whole. A demonstration of this is presented in Figure 6.5 using combs in the microwave region. Generally, filters that are applied to each individual chirped pulse affect the frequency profile of the comb as a whole, while filters applied to the entire comb affect the lineshape of the comb teeth.

Just as for chirped pulses, chirped pulse frequency combs can be multiplied 
(A) Micropulse Filtering
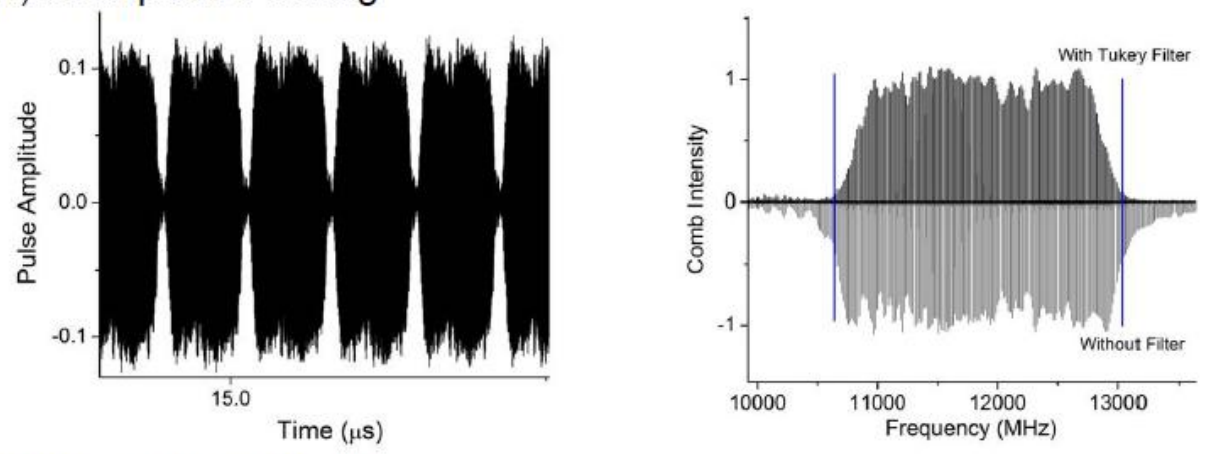

(B) Macropulse Filtering
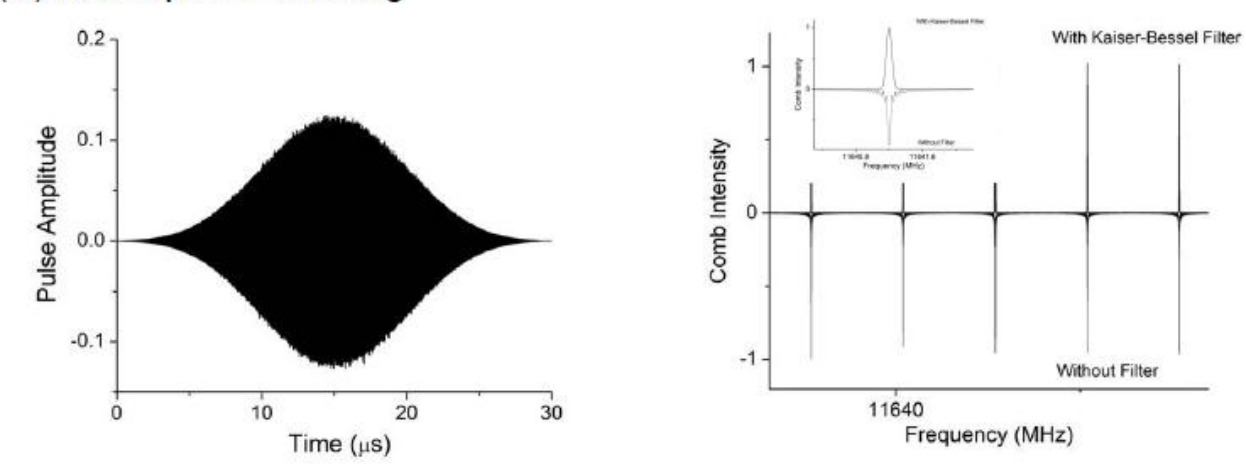

Figure 6.5. Application of digital filters to chirped-pulse frequency combs. Panel (A): filtering the individual chirped pulses with a tapered cosine (Tukey) window, which increases the sharpness of the decay of the "wings" of the overall comb. Panel (B): filtering the overall pulse train with a Kaiser-Bessel window, which reduces the sidelobes on the individual comb teeth. 
readily because the comb has a single frequency at any given point in time. This is shown in Figure 6.6, where a frequency comb from 13.5-18.0 GHz is doubled into a 27.0$36.0 \mathrm{GHz}$ comb. Expanded views on the right side show that while the bandwidth of the comb is doubled in the multiplication step, the spacing between the comb teeth is unchanged because the repetition rate of the comb is unchanged.

The detection bandwidth of this comb can be compressed by using a second frequency comb as the local oscillator for downconversion, rather than a single-frequency local oscillator. This multiheterodyne mixing technique is rooted in similar techniques with laser frequency combs. ${ }^{17-20}$ This is demonstrated at microwave frequencies in Figure 6.7. The basic concept is that the second comb covers the same bandwidth as the first, but has a faster repetition rate so that the comb teeth are more widely spaced (by $\left.v_{\text {mix }}\right)$. When the two combs are mixed, the resulting pulse contains contributions from each tooth of the signal comb mixing with each tooth of the mix comb, so each signal comb frequency is measured at several IF frequencies, the lowest of which is between 0 and $v_{\text {mix }} / 2$. The biggest challenge is devising a combination of repetition rates for the two combs so that the map between molecular and detection frequencies is one-to-one; that is, that no two downconverted comb teeth overlap.

In laser frequency combs, this is often accomplished by making the repetition rate of the mix comb only slightly offset from that of the signal comb. However, for largebandwidth millimeter-wave spectroscopy this is not practical: at typical Doppler linewidths of $\sim 1 \mathrm{MHz}$ (in the low-pressure limit), the desired repetition rate for the signal 

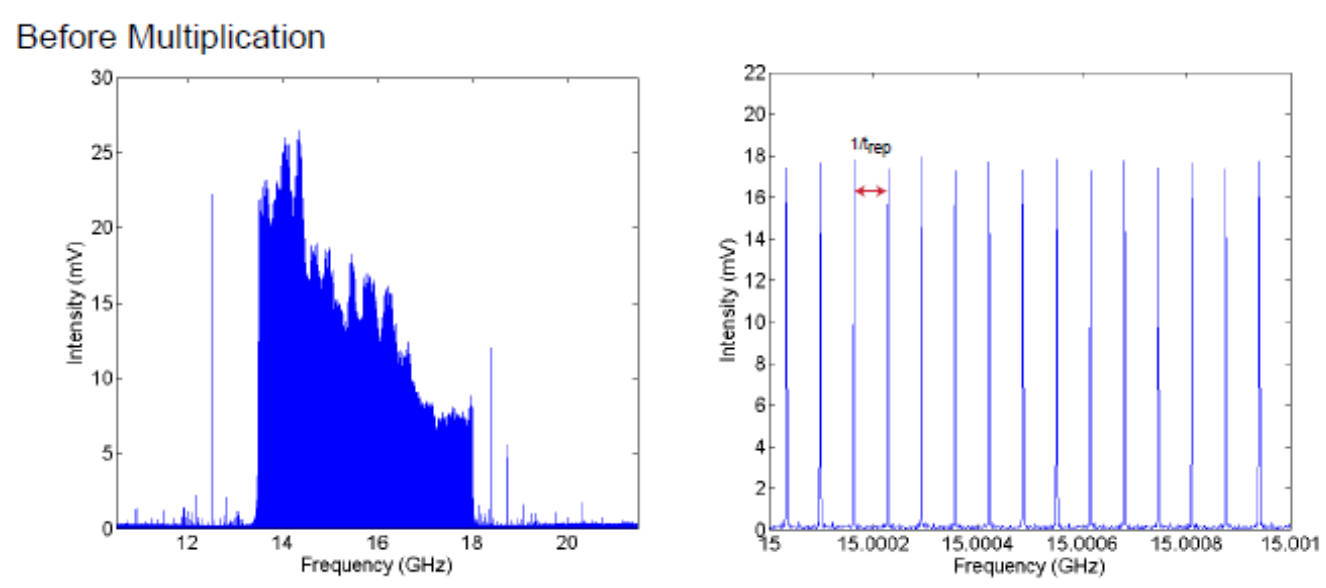

After Multiplication
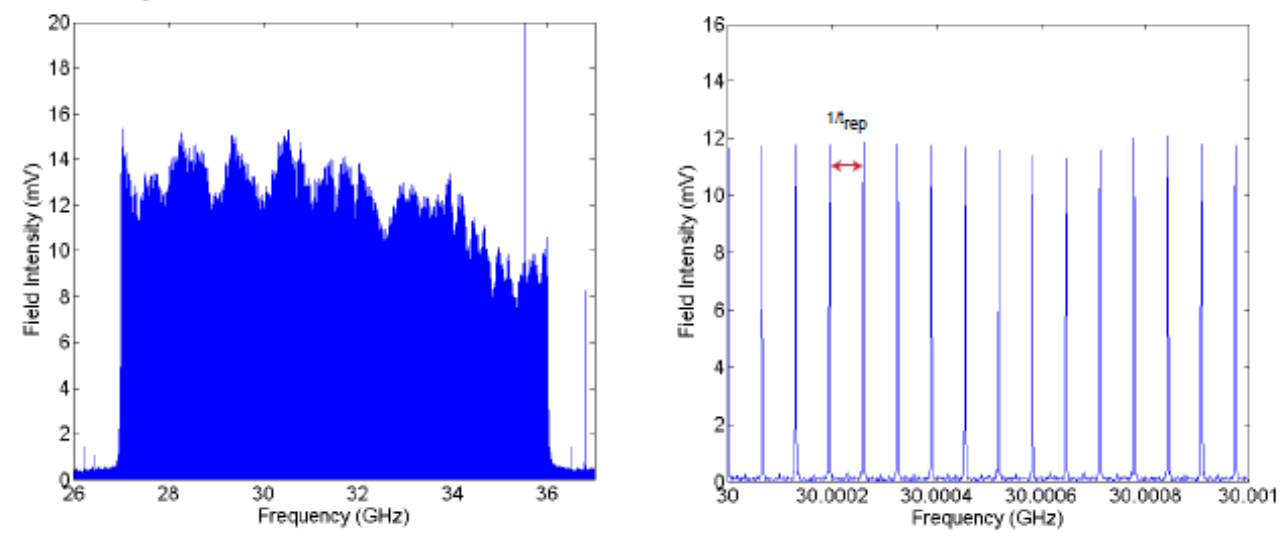

Figure 6.6. Frequency multiplication of a chirped-pulse frequency comb. The top panels shows the Fourier transform of a comb from 13.5-18.0 GHz, while the bottom panels show the same comb after being frequency doubled to 27.0-36.0 GHz. The right panels show expanded views of the combs, demonstrating that while the bandwidth is extended by the frequency multiplier, the spacing between comb teeth is unchanged. 


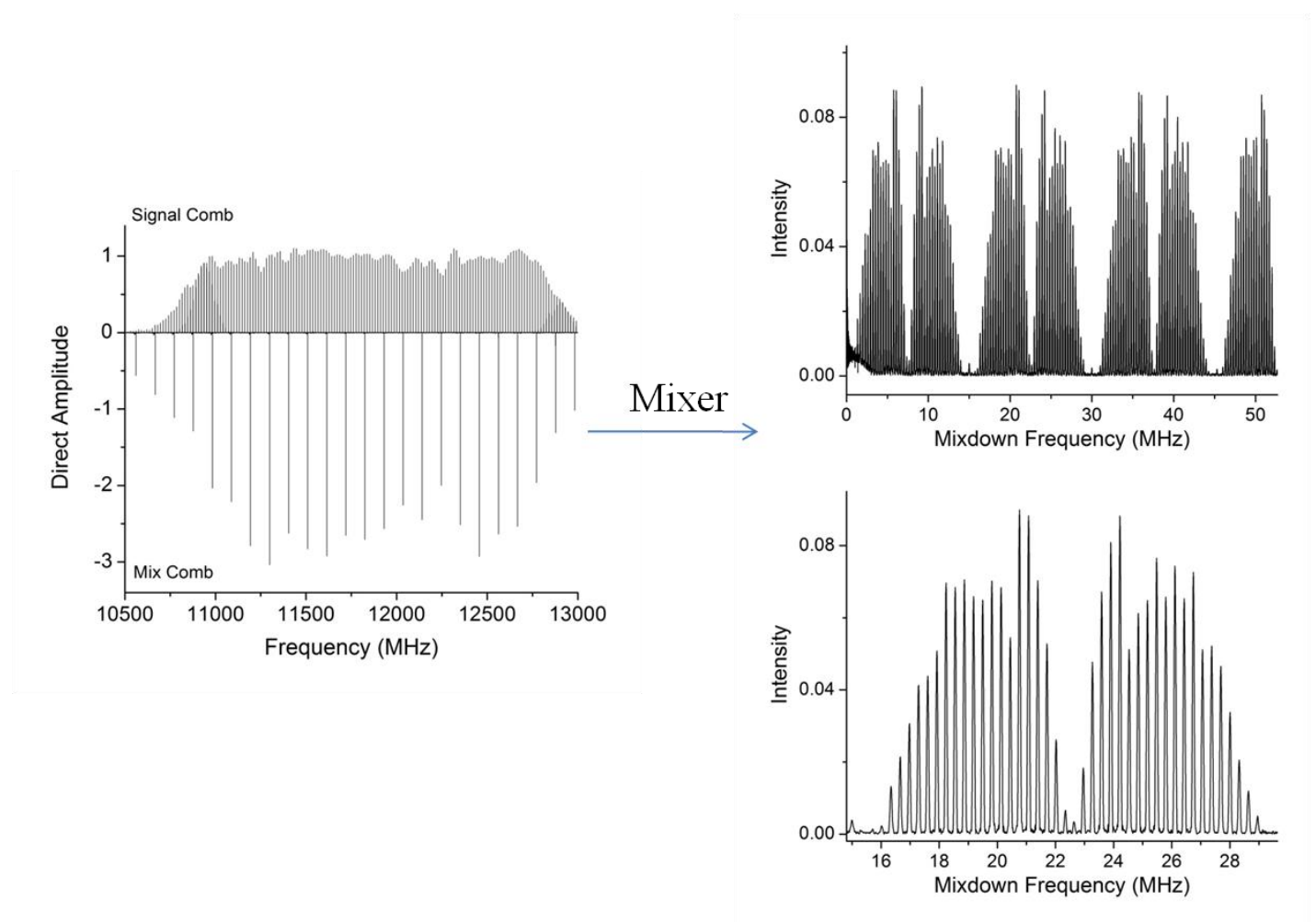

Figure 6.7. Demonstration of dual-comb mixing in a chirped-pulse frequency comb experiment. In this example, a $2.4 \mathrm{GHz}$ bandwidth comb (10.6-13.0 GHz) with comb spacing $15 \mathrm{MHz}$ (160 comb teeth) is mixed with a comb with spacing $105.2 \mathrm{MHz}$, and the mixed comb is measured in a detection bandwidth of $52.6 \mathrm{MHz}$. 
comb is on the order of $50 \mathrm{kHz}$ to get good lineshape determination. If the repetition rate of the mix comb is slightly offset from this, the comb would be detected in a $\sim 25 \mathrm{kHz}$ bandwidth; if the goal is to measure, say, $50 \mathrm{GHz}$ of spectral bandwidth (or $10^{6}$ frequencies), the linewidths of the comb teeth are far too broad (for roughly $1 \mathrm{~ms}$ duration combs) to measure this many teeth in such a small detection bandwidth. Instead, a mix comb frequency must be chosen that avoids overlaps. In a $10 \mathrm{~ms}$ comb, for example, the FWHM of each comb tooth is less than $1 \mathrm{kHz}$, so in principle $10^{6}$ frequencies can be measured in a few $\mathrm{GHz}$ of detection bandwidth. Note that this does not meet the desire stated above to measure spectra in an IF bandwidth of $500 \mathrm{MHz}$ or less, but this measurement can still be accomplished with a lower digitization rate than a single-frequency LO approach. As will be discussed below, the frequency comb approach becomes especially appealing in samples with elevated pressure, where the $T_{2}$ dephasing time is too short for measuring the free induction decay signal; in these cases, the frequency comb spacing can be made larger and the comb is more amenable to compression.

Two demonstrations of molecular absorption of a chirped-pulse frequency comb are presented in Figures 6.8 and 6.9. Figure 6.8 shows chirped-pulse absorption spectroscopy at microwave frequencies, using the $27.0-36.0 \mathrm{GHz}$ frequency comb created by a frequency doubler shown in Figure 6.6. For this measurement, a pulsed jet was used to work with the sample more easily, and comb compression was not performed. The low absorption of the sample $(\sim 1 \%)$ leads to far lower sensitivity than in a normal CP- 


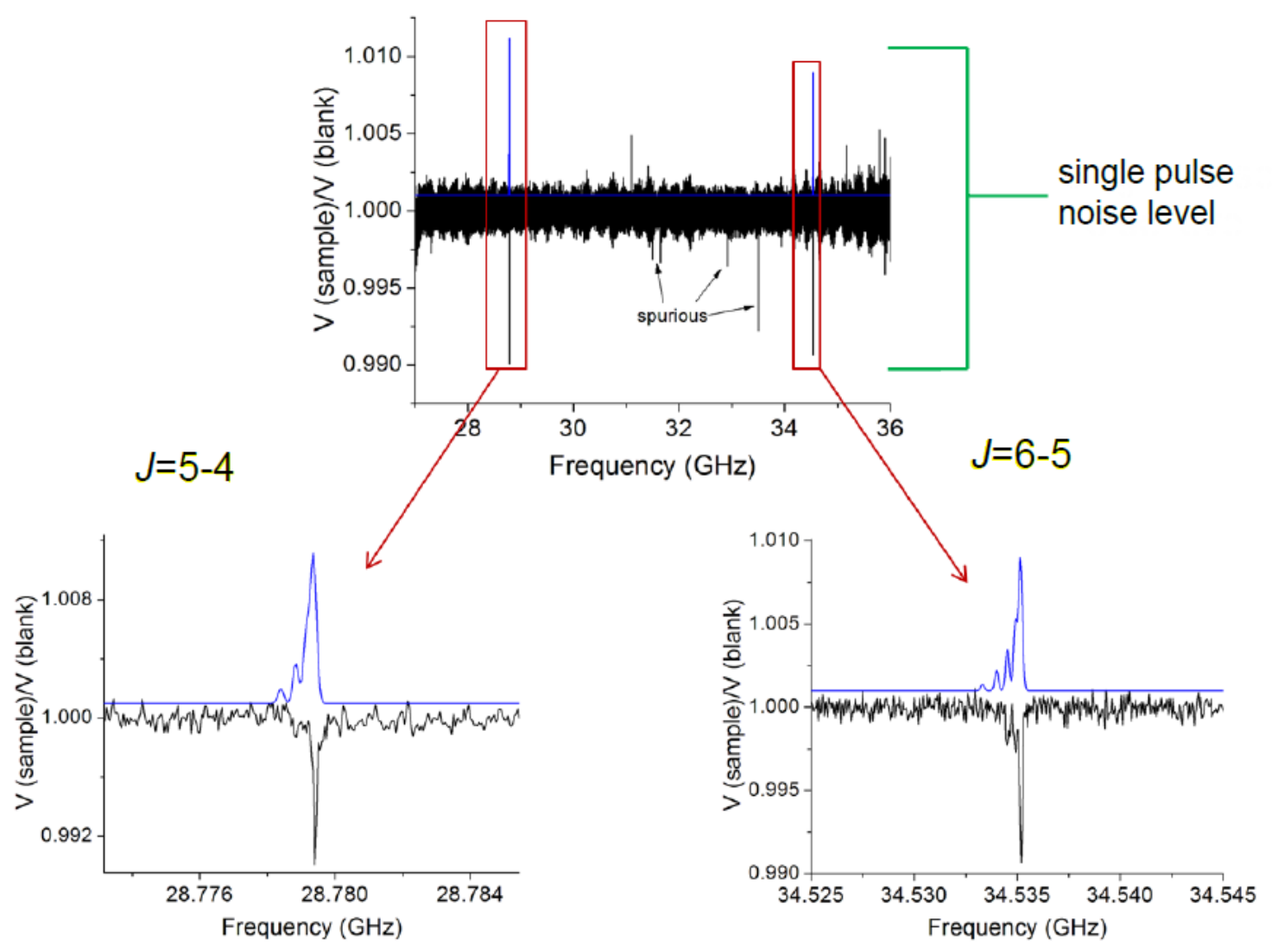

Figure 6.8. Demonstration of measuring molecular absorption with a chirped-pulse frequency comb on the spectrum of 3,3,3-trifluoropropyne from 27-36 GHz. In this spectrum 1,000 signal averages were acquired. Expanded views of the two sets of transitions in the range of the comb are shown. This spectrum was measured by detection with a single-frequency LO rather than with a second comb. 


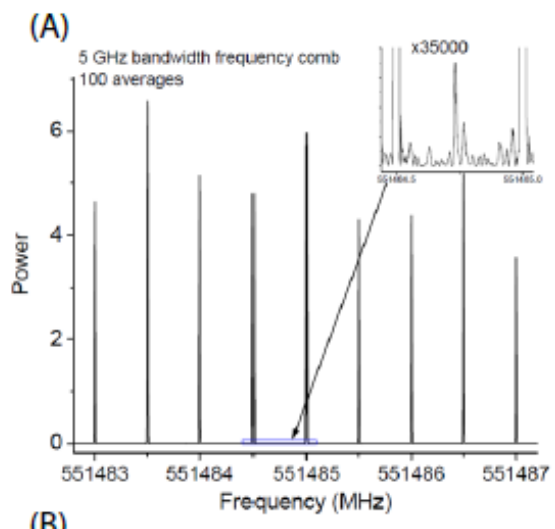

(B)
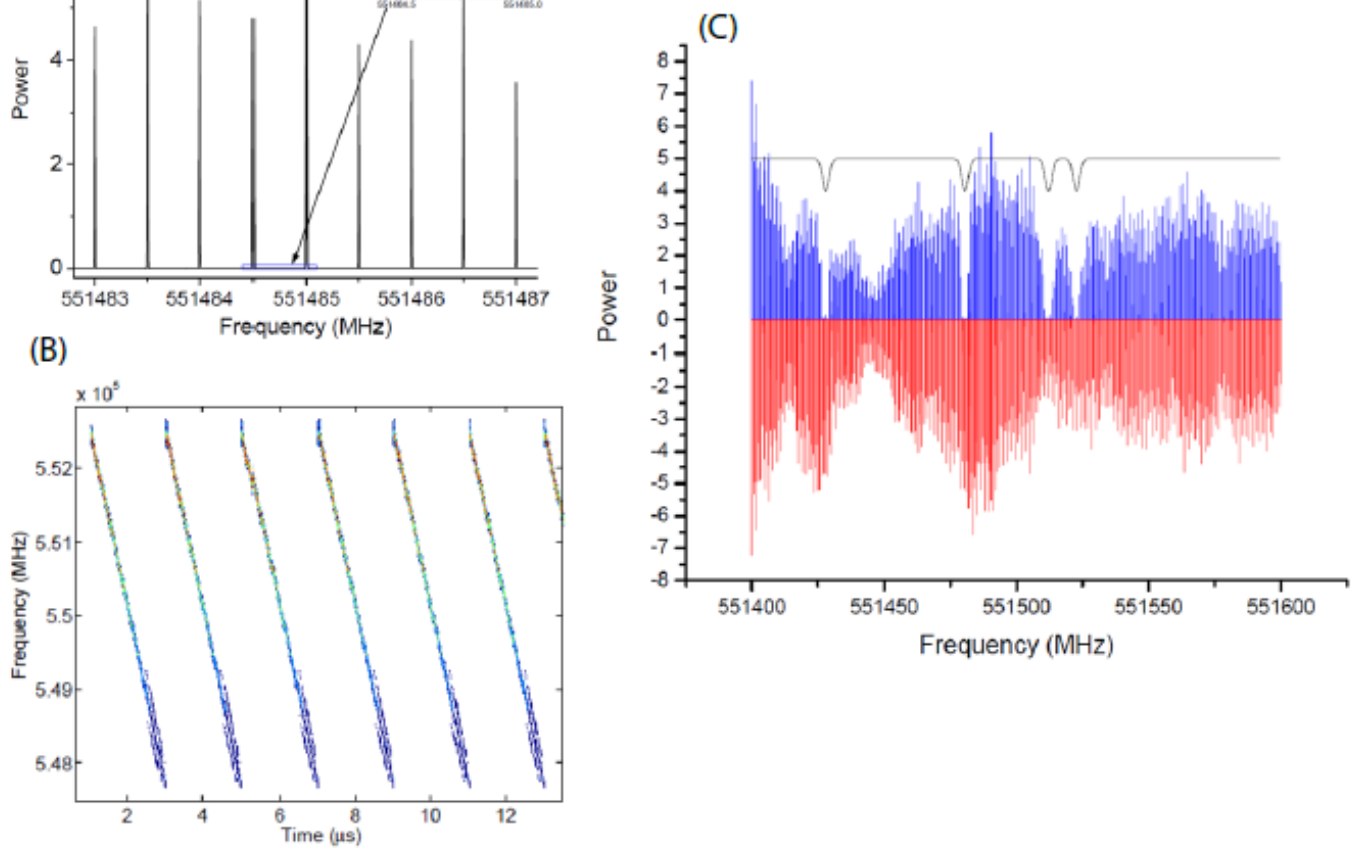

Figure 6.9. Demonstration of molecular absorption of a millimeter-wave frequency comb. Panel (A): The Fourier transform of the frequency comb, which had a bandwidth of $\sim 5 \mathrm{GHz}$ and was detected by heterodyne mixing with a single-frequency local oscillator. Panel (B): The spectrogram of the comb. Panel (C): Comparison of the comb after passing through a static gas sample of methyl cyanide at $298 \mathrm{~K}$ (blue) with one passing through a blank cell (red). The cell pressure was 9 mTorr. 
FTMW emission experiment (for comparison, the transitions observed in this spectrum would have a signal to noise of roughly 100:1 on a single shot). The noise level after 1,000 averages can be seen to be roughly $0.1 \%$.

In Figure 6.9 a millimeter-wave chirped-pulse frequency comb from 548-553 $\mathrm{GHz}$ is shown. This comb was likewise detected by heterodyne detection with a singlefrequency local oscillator, without bandwidth compression. The panels on the left show that the background comb, even after multiplication (in this case, $\mathrm{x} 48$ ), has very high spectral purity. On the right, combs with and without gas in the cell are compared. A portion of the spectrum of methyl cyanide is presented in panel (C) on the right, showing that at the frequencies of the four molecular transitions in this $200 \mathrm{MHz}$ region, the amplitude of the frequency comb is reduced to zero; these transitions are saturated in this spectrum.

\section{Segmented Chirped-Pulse Fourier Transform Emission Spectroscopy}

Rather than measuring molecular transitions through their absorption of radiation, molecular features can also be measured by applying a brief $\left(<T_{2}\right)$ excitation pulse, followed by the background-free detection of transitions through their free induction decay. This experiment works the same way as in the microwave, except $T_{2}$ times are shorter at higher frequencies and therefore the excitation pulses must be shorter (typically $100 \mathrm{~ns}$ or less at $500 \mathrm{GHz}$ ). Implementations of this technique at millimeter wave frequencies, using excitation pulses with bandwidths of $\sim 10 \mathrm{GHz}$ and downconverting

with a single-frequency local oscillator, have been presented. ${ }^{11,12}$ In order to measure 
spectra with far higher bandwidths $(\geq 30 \mathrm{GHz})$ with this technique, the technique of segmented CP-FT spectroscopy has been developed. The basic principle of this technique is presented in Figure 6.10. The primary difference is that the second channel of the AWG is used to downconvert the molecular emissions, rather than a singlefrequency oscillator. Therefore, the frequency of the local oscillator can be changed quickly. The spectrum can therefore be "segmented" where in each segment, a small bandwidth is polarized, followed by the FID detection of all the molecular transitions in the bandwidth of the pulse. The local oscillator frequency for each segment is chosen to be slightly offset from the frequency range of the pulse.

It should be evident that there are few limitations on the number and bandwidth of segments that can be used in these measurements. This technique is particularly amenable to detection on a $500 \mathrm{MHz}$ oscilloscope. Because of the low power levels available at this frequency range compared to in the microwave, signals for most molecules are in the weak pulse limit; that is, the signals are proportional to $(\Delta v)^{-1 / 2}$, where $\Delta v$ is the bandwidth of the segment polarization pulse. ${ }^{21}$ When a large-bandwidth spectrum is broken into $N$ segments, this means that because the bandwidth of each segment is reduced by $N$ over a single polarization pulse, the signal to noise of all transitions is increased by $\sqrt{N}$, which compensates for the factor of $N$ longer that it requires to measure the whole spectrum (since $N$ signal averages would be required at full bandwidth to recover the same sensitivity). Therefore, in the weak pulse limit, segmenting the spectrum does not affect the sensitivity per measurement time. 

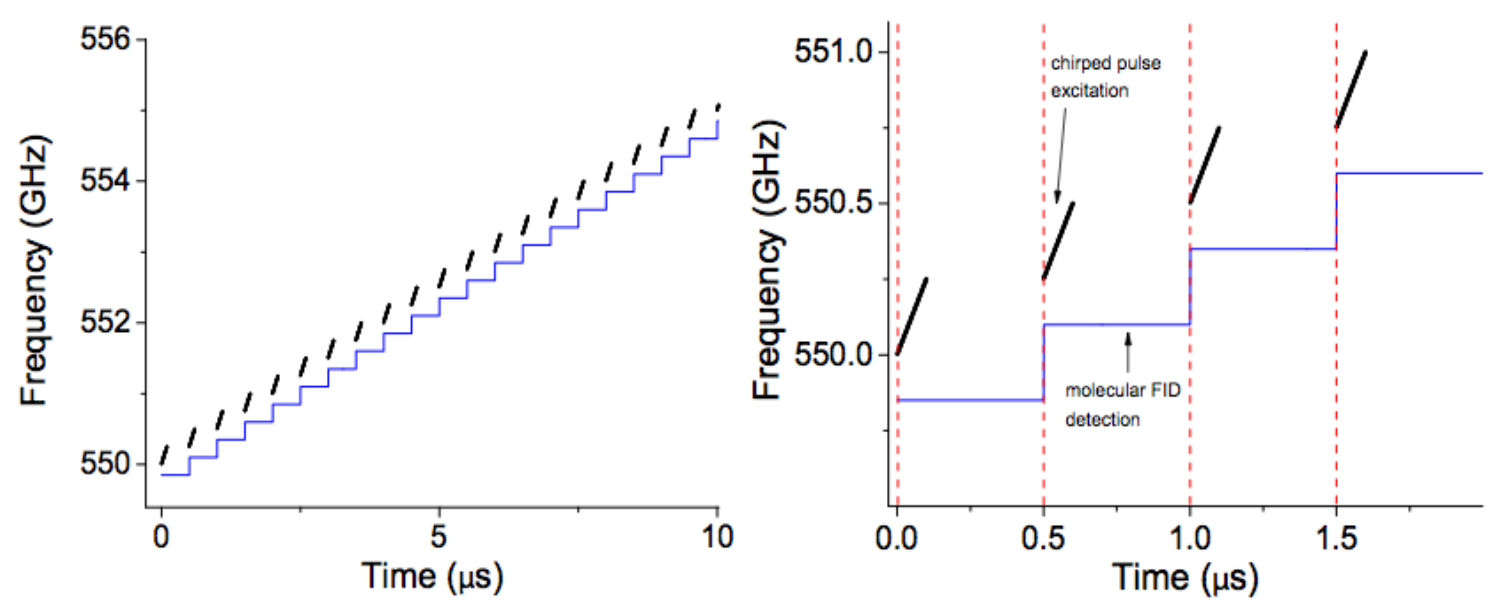

Figure 6.10. Example spectrogram for a segmented chirped-pulse Fourier transform experiment. The black trace indicates the channel for chirped pulse excitation (which is transmitted through the molecular sample) and the blue trace indicates the local oscillator. 
The bandwidth of the spectrum that can be acquired by segmented CP-FT spectroscopy is limited only by the bandwidth of the AMCs and the heterodyne detector. While segmenting does not offer an improvement in the sensitivity per unit of measurement time, the reduction in digitization rate means that many fewer data points are required to obtain the same amount of information. Because most chirped-pulse spectroscopic techniques are data throughput limited, this can offer a significant enhancement in terms of sensitivity per unit of lab time. Additionally, as mentioned above, this technique can measure higher bandwidth spectra than can be measured on currently commercially available digital oscilloscopes.

A measurement of the spectrum of methanol using the segmented CP-FT emission technique is presented in Figure 6.11. This spectrum was run at elevated pressure for two reasons: to minimize the total measurement time, and to reduce the impact of an echo in the White cell used for the measurement. This echo occurs $167 \mathrm{~ns}$ after the original polarization pulse, corresponding to the $50 \mathrm{~m}$ additional round-trip pathlength that the echo pulse follows before reaching the receiver. Therefore, this measurement was run with a cell pressure of 40 mTorr, so that the entire measurement takes place before the echo pulse reaches the detector. This figure shows that segmented CP-FT spectroscopy has good frequency and relative intensity accuracy, with extremely fast measurement time (as fast as $1 \mathrm{GHz}$ per $\mu \mathrm{s}$ ).

The absorption and emission techniques have complementary strengths and would likely both be useful for different samples. The primary difference between the two 


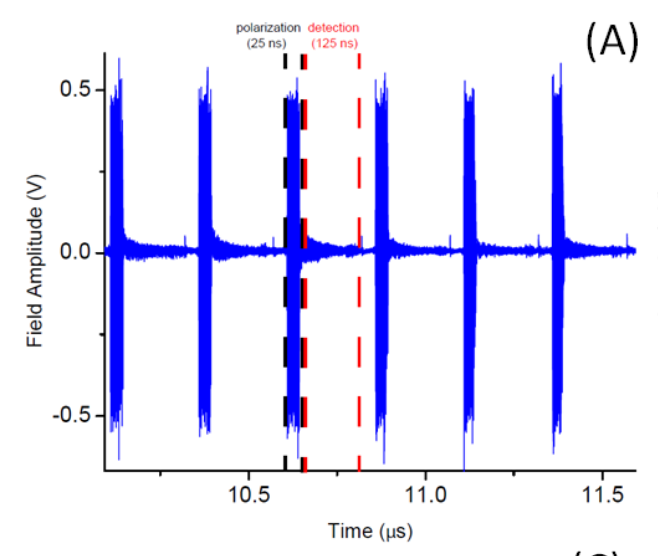

(C)
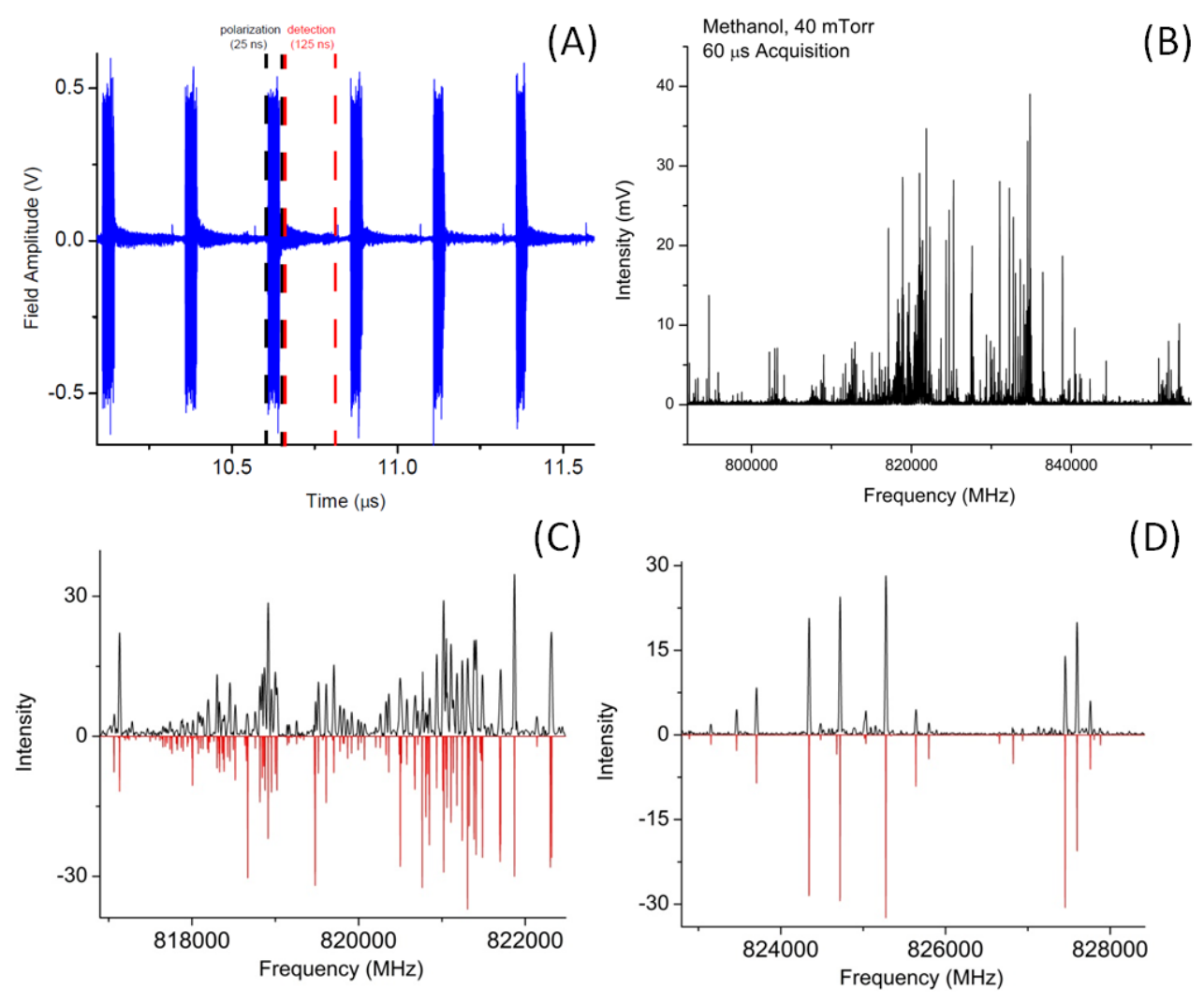

(D)

Figure 6.11. Segmented CP-FT spectrum of methanol from 790-860 GHz. This spectrum is a single acquisition with a total measurement time of $60 \mu \mathrm{s}$. Each segment had a total duration of $250 \mathrm{~ns}$ : $25 \mathrm{~ns}$ for chirped pulse excitation, $125 \mathrm{~ns}$ for FID detection, and a $100 \mathrm{~ns}$ delay for LO frequency switching. Each segment had a bandwidth of $288 \mathrm{MHz}$. Panel (A) shows a $1.5 \mu$ s portion of the time domain spectrum, acquiring six segments $(1732 \mathrm{MHz})$. Panel (B) shows the frequency spectrum with all of the 232 segments concatenated. Panels (C) and (D) show expanded views of the spectrum compared to a simulation from line parameters from the JPL spectral catalog. 
techniques is that the absorption technique does not require narrow linewidths. For emission, broader features correspond to fast $T_{2}$ decay times, which eventually prevents detection in this way because there is an instrumentally limited dead time between molecular polarization and FID detection. For lower-pressure samples where the dephasing times are longer, however, it is anticipated that emission techniques will achieve higher sensitivity: the absorption and emission signals are in theory of equal amplitude, but the absorption signal must be measured against the excitation pulse while emission can be measured background-free. In support of this observation, a recent publication demonstrating multiheterodyne absorption spectroscopy with frequency combs in the near-infrared ${ }^{17}$ was followed by a report using the same demonstrating an order of magnitude sensitivity enhancement by instead detecting the time-domain FID signal. $^{22}$ In practice, as the two techniques can both be performed with a single spectrometer design, both are likely to be useful for different sample conditions.

An additional piece of information that could prove to be a powerful one for the characterization of complex mixtures comes from characterizing the decay profile of the molecular transitions. For a static cell sample, the decay typically contains contributions from collisional broadening (which has a Lorentzian profile) and Doppler broadening (which has a Gaussian profile). If these two decay rates can be determined through a non-linear least squares fit of the time-domain free induction decay signal, the pressure broadening coefficient can be determined if the sample pressure is known, and the mass of the molecule can be determined if the temperature is known. This is a powerful application for the characterization of unknown species; this can serve as a sort of double 
resonance for extracting spectral assignments from a mixture. This analysis is challenging due to the high degree of correlation between Gaussian and Lorentzian components. This correlation gets higher as the time between the polarization of the transition and the start of the FID measurement increases. The echo in the White cell has so far prevented the acquisition of a spectrum where the Lorentzian and Gaussian components can be fit independently. This type of time-domain analysis has been previously explored. ${ }^{23,24}$

\section{Conclusions and Future Directions}

This chapter offers only a brief summary into a field that has the promise to be a revolutionary technique for molecular spectroscopy in the millimeter/submillimeter region. There are a number of aspects of the experimental design that remain to be tested. The optimal cell design is one of the design components that deserves further attention. The $25 \mathrm{~m}$ White cell employed in the millimeter-wave measurements presented here offers a long pathlength, but the echo that has yet to be removed from the system is highly undesirable and disrupts several aspects of the measurement, reducing sensitivity, increasing linewidth, and complicating the potential for mass resolution discussed above. Additionally, the considerable transmission loss (it is estimated that only $\sim 20 \%$ of the radiation reaches the detector) could negate the benefit of the long pathlength. A folded 4-6 m pathlength cell will be tested at UVa which could offer similar sensitivity without the echo and with simpler alignment. This cell would have approximately the same pathlength as the FASSST and JPL instruments. 
For the absorption measurement, the biggest challenge is the fluctuation of the amplitude of the background pulse. This can lead to large modulations in the $E_{\text {sample }} / E_{\text {blank }}$ spectrum that are periodic and can obscure molecular transitions. Because chirped-pulse frequency combs have a programmable frequency offset, a modulation technique could be instituted to separate intensity dips due to molecular transitions from the changes in the comb background intensity that vary more slowly with frequency. Additionally, the best technique to use for comb detection remains to be explored: dualcomb mixing offers the promise of large bandwidth compression factors, but the power of the comb is split among multiple channels, and the noise from mixing with each tooth of the mix comb adds in IF space, so there may be another detection approach with higher sensitivity.

For emission spectroscopy, one significant experimental challenge going forward is the recovery of the natural absorption linewidth. Two modifications are needed to accomplish this. The first is that the dead time, particularly the pulse echo in the cell, needs to be eliminated; because the Doppler decay rate increases as $t-t_{0}$ increases (where $t_{0}$ is the time of polarization), the transition linewidth increases if there is dead time in the system. Secondly, the CP-FT spectra presented throughout this thesis, including in the submillimeter as in Figure 6.11, are magnitude Fourier transforms (that is, they contain both absorption and dispersion components). If only the absorption component is extracted, the linewidth of the transitions decreases by a factor of $\sqrt{3}$. This recovery is challenging due to the fast phase variation of the chirped pulse. Work is being done by the NIST group to attempt to automate the real Fourier transform determination. ${ }^{25}$ One 
approach that could offer promise is in the measurement of the chirped pulses as well as the FIDs. The phase of the molecular emissions is exactly $180^{\circ}$ out of phase with that of the excitation pulse when it crosses the molecular absorption frequency. Therefore, from the phase of the chirped pulse (which can in theory be determined by a fitting algorithm, if it is measured), the phase of molecular transitions could be determined. Because the FIDs are typically considerably weaker than the chirped pulses, this might require a second measurement because the pulses and molecular FIDs require different digitizer scales. Alternatively, a receiver design has been proposed that splits the chirped pulses and FIDs and amplifies the FIDs to bring them onto the same digitizer scale; it is not yet known whether the phase correspondence of the pulses and FIDs is preserved when the two pass through differing components; this is another aspect where the low detection bandwidth on each segment may be beneficial.

While there are a number of design parameters that remain to be optimized, this spectrometer offers promise as a powerful instrument for the broadband detection of large bandwidth millimeter/submillimeter spectra. For commercial applications, the most alluring feature is the potential for the monitoring of multiple molecular concentrations in the gas phase, with video frame rate time resolution. Because the system consists only of solid-state components, and the pumping requirements are fairly minimal, a compact and portable spectrometer design is possible. Because of the unique nature of molecular rotational spectroscopy, where the spectral signature of each molecule is relatable to accurately calculable Hamiltonian parameters, library-free characterization can be accomplished using this spectrometer; that is, the structures of molecules for which 
laboratory spectra are not known can be determined by comparison to a theoretical molecular constant database. Using the techniques presented in Chapter 3, particularly double resonance spectroscopy and automated triples fitting, this molecular assignment process could be completely automated. This spectrometer also yields great promise in the measurement of the spectra of astrophysically relevant molecules due to its bandwidth and sensitivity, and in monitoring the concentrations of transient species such as reaction intermediates or the products of a discharge. 


\section{Chapter 6 References}

(1) De Lucia, F. C. J. Mol. Spectrosc. 2010, 261, 1-17.

(2) Herbst, E.; van Dishoeck, E. F. Annu. Rev. Astron. Astrophys. 2009, 47, 427-480.

(3) Drouin, B. J.; Maiwald, F. W.; Pearson, J. C. Rev. Sci. Instrum. 2005, 76, 093113093110.

(4) Ziurys, L. M.; Barclay, J. W. L.; Anderson, M. A.; Fletcher, D. A.; Lamb, J. W. Rev. Sci. Instrum. 1994, 65, 1517-1522.

(5) Hepp, M.; Jäger, W.; Pak, I.; Winnewisser, G. J. Mol. Spectrosc. 1996, 176, 5863.

(6) Mollaaghababa, R.; Gottlieb, C. A.; Thaddeus, P. J. Chem. Phys. 1993, 98, 968973.

(7) Medvedev, I. R.; Neese, C. F.; Plummer, G. M.; De Lucia, F. C. Opt. Lett. 2010, $35,1533-1535$.

(8) Albert, S.; Petkie, D. T.; Bettens, R. P. A.; Belov, S. P.; Lucia, F. C. D. Anal. Chem. 1998, 70, 719A-727A.

(9) Petkie, D. T.; Goyette, T. M.; Bettens, R. P. A.; Belov, S. P.; Albert, S.;

Helminger, P.; De Lucia, F. C. Rev. Sci. Instrum. 1997, 68, 1675-1683.

(10) Fortman, S. M.; Medvedev, I. R.; Neese, C. F.; De Lucia, F. C. Astrophys. J. 2010, $725,1682-1687$.

(11) Park, G. B.; Steeves, A. H.; Kuyanov-Prozument, K.; Neill, J. L.; Field, R. W. J. Chem. Phys. 2011, in press. 
(12) Gerecht, E.; Douglass, K. O.; Plusquellic, D. F. Opt. Express 2011, 19, 89738984.

(13) Tobin, D. C.; Strow, L. L.; Lafferty, W. J.; Olson, W. B. Appl. Opt. 1996, 35, 4724-4734.

(14) Podobedov, V. B.; Plusquellic, D. F.; Siegrist, K. E.; Fraser, G. T.; Ma, Q.;

Tipping, R. H. J. Quant. Spectrosc. Rad. Trans. 2008, 109, 458-467.

(15) Adler, F.; Thorpe, M. J.; Cossel, K. C.; Ye, J. Annu. Rev. Anal. Chem. 2010, 3, $175-205$.

(16) Maddaloni, P.; Cancio, P.; De Natale, P. Meas. Sci. Tech. 2009, 20, 052001.

(17) Coddington, I.; Swann, W. C.; Newbury, N. R. Phys. Rev. Lett. 2008, 100, 013902.

(18) Schliesser, A.; Brehm, M.; Keilmann, F.; van der Weide, D. Opt. Express 2005, 13, 9029-9038.

(19) Keilmann, F.; Gohle, C.; Holzwarth, R. Opt. Lett. 2004, 29, 1542-1544.

(20) Schiller, S. Opt. Lett. 2002, 27, 766-768.

(21) Brown, G. G.; Dian, B. C.; Douglass, K. O.; Geyer, S. M.; Shipman, S. T.; Pate, B. H. Rev. Sci. Instrum. 2008, 79, 053103-053113.

(22) Coddington, I.; Swann, W. C.; Newbury, N. R. Opt. Lett. 2010, 35, 1395-1397.

(23) Coerdt, R.; Grönig, H. Appl. Opt. 1989, 28, 3021-3023.

(24) Whittenburg, S. L. Spectrochim. Acta A 1996, 52, 1169-1174.

(25) Gerecht, E.; Douglass, K. O.; Plusquellic, D. F. 66th International Symposium on Molecular Spectroscopy Columbus, OH, 2011, RB08. 
(26) Available on the Web at http://spec.jpl.nasa.gov/ftp/pub/catalog/catform.html. 


\section{Chapter 7}

\section{Conclusions}

\section{Summary}

This thesis has presented the development of techniques for high-sensitivity chirped pulse molecular rotational spectroscopy in the microwave and millimeter wave regions. Two applications of this technique have been presented. First, the high sensitivity of CP-FTMW spectroscopy facilitates the direct structural determination of biologically relevant molecules and weakly bound molecular complexes. This direct structural information comes from the measurement of isotopically labeled species. Because isotopically labeled samples of biological molecules are usually difficult and/or expensive to synthesize, the high sensitivity of CP-FTMW enables the detection of minor isotopomers in natural abundance, even for a molecule with 15 heavy atoms (strawberry aldehyde). This brings molecular rotational spectroscopy, which has typically been limited to smaller species for which electronic structure theory is generally very accurate, into the size regime where weak interactions that are harder to characterize computationally become significant, and potential energy surfaces become extremely complex. Therefore, the direct structural information that CP-FTMW spectroscopy can provide for molecules of this size is a powerful tool to directly test electronic structure theory and aid the development of new theoretical methods.

Secondly, the application of CP-FTMW spectroscopy to interstellar chemistry has been presented. The next generation of radio astronomical observatories will offer an unprecedented view of the chemistry of star-forming regions of our galaxy, in terms of 
sensitivity, spatial resolution, and spectral resolution. The spectra that come from these observatories contain the signatures of a large number of transitions that are yet to be assigned (both transitions of molecules that have already been found in the interstellar molecules, and of molecules whose laboratory spectra are yet to be measured). In order to thoroughly characterize the chemical composition of these regions, targeted broadband laboratory searches for molecules that could be formed based on proposed chemical pathways in the interstellar medium are needed. Using spatial distribution information from two of these observatories, the higher-energy conformational isomer of methyl formate was proposed as a possible interstellar species. The rotational spectrum of this conformer was then detected for the first time by CP-FTMW spectroscopy, based on which it was detected for the first time in the interstellar medium. This detection in turn suggests several possible reaction pathways that can be studied by observational, theoretical, and experimental methods. This basic approach should provide a powerful way to extract chemical information from the radio astronomical spectra that will be available in the coming years.

\section{Future Developments}

In the next few years, the sensitivity and measurement speed of chirped pulse spectrometers will be enhanced by further developments in technology. The major anticipated advance is a faster digital oscilloscope that can more efficiently process data in the Fast Frame mode. As discussed in Chapter 3, for molecular-beam CP-FTMW spectroscopy, the pulsed nozzles in the UVa instrument are currently limited to a 
repetition rate of $0.7 \mathrm{~Hz}$ (for a $50 \mathrm{GS} / \mathrm{s}$ digitization rate, with $20 \mu$ s FID detection time, and 10 FIDs acquired per valve cycle, for a total of $10^{7}$ data points acquired per pulse). This is far slower than the current vacuum pumps can handle. Once the data throughput improves so that the repetition rate is pump-limited $(\sim 10 \mathrm{~Hz})$, the spectrum of strawberry aldehyde with isotopomer sensitivity presented in Figure 3.5, for example, would require only $2.5 \mathrm{hr}$ to acquire. For static gas experiments, such as the chirped-pulse millimeter wave technique, there are no constant pumping requirements and so the repetition rate can be almost continuous (with the exception of possible limitations in power handling or switching of control components).

The coupling of chirped pulse spectroscopy to sources of transient or reactive species has been explored by several groups. The use of a molecular discharge has been reported in this thesis to generate the trans conformations of methyl and ethyl formate, and can also be used to measure the spectra of reactive species, as has been demonstrated in the past few years. ${ }^{1,2}$ The development of laser-ablation chirped-pulse FTMW spectroscopy has also been reported recently ${ }^{3}$ and is an important technique for measuring the spectra of heavier biomolecules, because many of these molecules undergo decomposition when heated. The broadband nature of chirped pulse spectroscopy means that even when the sample conditions are changing rapidly, the full-band spectra of a discharged or ablated sample can be measured with very low sample consumption.

As has been discussed previously in this thesis, chirped-pulse rotational spectroscopy can be used to perform library-free characterization of complex chemical mixtures, due to the relationship between the observed spectrum and theoretically 
calculable parameters, primarily the molecular shape. The techniques that have been presented in this thesis to aid in the spectral assignment of the dense broadband spectra that often result from chirped-pulse instruments can be used along with a theoretical rotational constant database to develop an instrument that can output the structures of all of the molecules in a mixture, along with their relative concentrations, even if species in the sample have never been measured before in the laboratory. The continued optimization of double resonance and automated fitting techniques in the coming years will be needed for the development of what will be an extremely powerful and broadly useful instrument for chemical analysis. 


\section{Chapter 7 References}

(1) McCarthy, M. C.; Gottlieb, C. A.; Thaddeus, P.; Muckle, M. T.; Neill, J. L.; Pate, B. H.; Brunken, S.; Belloche, A.; Verheyen, L.; Menten, K. M.; Remijan, A. J. 64th International Symposium on Molecular Spectroscopy Columbus, OH, 2009, WI06.

(2) Zaleski, D. P.; Neill, J. L.; Muckle, M. T.; Steber, A. L.; Pate, B. H.; Corby, J. F.; Lattanzi, V.; McCarthy, M. C.; Remijan, A. J. 66th International Symposium on Molecular Spectroscopy Columbus, OH, 2011, WF01.

(3) Mata, S.; Pena, I.; Cabezas, C.; Lopez, J. C.; Alonso, J. L.; Pate, B. H. 66th International Symposium on Molecular Spectroscopy Columbus, OH, 2011, RC05. 